César Yukishigue Kiyono

Projeto de Transdutores Piezocompósitos de Casca Multi-Camada utilizando o Método de Otimização Topológica 
César Yukishigue Kiyono

\section{Projeto de Transdutores Piezocompósitos de Casca Multi-Camada utilizando o Método de Otimização Topológica}

Tese apresentada à Escola Politécnica da Universidade de São Paulo para obtenção do Título de Doutor em Engenharia Mecânica. 
César Yukishigue Kiyono

\section{Projeto de Transdutores Piezocompósitos de Casca Multi-Camada utilizando o Método de Otimização Topológica}

Tese apresentada à Escola Politécnica da Universidade de São Paulo para obtenção do Título de Doutor em Engenharia Mecânica.

Área de concentração:

Engenharia de Controle e Automação Mecânica

Orientador:

Prof. Dr. Emílio Carlos Nelli Silva 
Este exemplar foi revisado e corrigido em relação à versão original, sob responsabilidade única do autor e com a anuência de seu orientador.

São Paulo, de março de 2013.

Assinatura do autor

Assinatura do orientador

FICHA CATALOGRÁFICA

\section{Kiyono, Cesar Yukishigue}

Projeto de transdutores piezocompósitos de casca multicamada utilizando o método de otimização topológica / C.Y. Kiyono. - versão corr. -- São Paulo, 2013.

$170 \mathrm{p}$.

Tese (Doutorado) - Escola Politécnica da Universidade de São Paulo. Departamento de Engenharia Mecatrônica e de Sistemas Mecânicos.

1. Métodos topológicos (Otimização) 2. Atuadores piezelétricos 3. Sensor 4. Energia (Coleta) 5. Método dos elementos finitos I.Universidade de São Paulo. Escola Politécnica. Departamento de Engenharia Mecatrônica e de Sistemas Mecânicos II. t. 
Dedico este trabalho à minha família.

"Deus ajuda, mas é você que vai fazer o trabalho!" (Rousimar "Toquinho" Palhares) 


\section{Agradecimentos}

À Deus, por oferecer mais esta oportunidade em minha vida.

Ao orientador, Prof. Dr. Emílio Carlos Nelli Silva, pela atenção, dedicação, paciência e empenho para a realização desse trabalho.

À minha querida esposa Patrícia, que tem sido muito paciente nos momentos de correria. E pelos ótimos momentos que temos juntos. Ao meu recém chegado filho, Vítor, que iluminou minha vida nessa última etapa do doutorado.

Ao professor J. N. Reddy pela colaboração na publicação de um artigo.

À FUSP (Fundação de Apoio à Universidade de São Paulo), pelo apoio financeiro deste trabalho, através da bolsa de doutorado. Ao CNPq (Conselho Nacional de Desenvolvimento Científico e Tecnológico) pelo apoio financeiro das viagens a congressos.

Aos meus pais, que sempre estiveram ao meu lado, física ou espiritualmente, dando todo tipo de suporte que eu necessitava, e por serem modelos de perseverança e disciplina, o que sempre me motivou a continuar. Aos meus tios Roberto e Elza, que cuidaram de mim em todos esses anos de faculdade enquanto estava em São Paulo.

Aos todos os amigos pessoais, de academia e de pesquisa, pelos ótimos momentos de convivência, de amizade, de fraternidade, e de risadas.

E por fim, a todos que direta ou indiretamente contribuíram para a realização deste trabalho. 


\section{Resumo}

Transdutores baseados em cascas piezocompósitas têm uma vasta aplicação no campo de estruturas inteligentes, principalmente como atuadores, sensores e coletores de energia. Essas estruturas piezocompósitas são geralmente compostas por dois ou mais tipos de materiais, como por exemplo materiais piezelétricos, ortotrópicos elásticos (possuem fibras de reforçamento) e isotrópicos (materiais homogêneos). Vários fatores devem ser considerados no projeto de transdutores baseados em cascas piezocompósitas, como o tamanho, a forma, a localização e a polarização do material piezelétrico, bem como a orientação das fibras do material ortotrópico. O projeto desses transdutores é complexo e trabalhos anteriores envolvendo esses tipos de materiais sugerem utilizar Método de Otimização Topológica (MOT) para aprimorar o desempenho dos transdutores distribuindo o material piezelétrico sobre substratos fixos de materiais isotrópicos e ortotrópicos, ou otimizar a orientação das fibras dos materiais ortotrópicos com material piezelétrico com tamanho, forma e localização previamente estabelecidos. Assim, nesta tese, propõe-se o desenvolvimento de uma metodologia baseada no MOT para projetar transdutores piezocompósitos de casca considerando, simultaneamente, a otimização da distribuição e do sentido de polarização do material piezelétrico, e também a otimização da orientação das fibras de materiais ortotrópicos, que é livre para assumir valores diferentes ao longo da mesma camada compósita. Utilizando essa metodologia, são obtidos resultados numéricos para atuadores e sensores em regime estático e para coletores de energia com circuito elétrico acoplado, em regime dinâmico amortecido. Para os casos dos sensores e dos coletores de energia, também são consideradas as tensões mecânicas na estrutura, as quais devem obedecer os critérios de von Mises (para materiais isotrópicos) e de Tsai-Wu (para materiais ortotrópicos) para que não haja falhas na estrutura, que está sujeita a esforços mecânicos.

Palavras-chave: Otimização Topológica, Transdutores Piezelétricos, Cascas Piezocompósitas, Orientação de Fibras, Coletores de Energia, Restrição de Tensão Mecânica. 


\section{Abstract}

Transducers based on laminated piezocomposite shell structures have a wide application in the field of smart structures, especially as actuators, sensors and energy harvesting devices. These piezocomposite structures are generally composed by two or more kinds of materials, such as piezoelectric, isotropic, and elastic orthotropic (fiber reinforcement) materials. Several factors must be considered in the design of piezocomposite transducers, such as size, shape, location and polarization of the piezoelectric material and the fiber orientation of the orthotropic material. The design of these transducers is complex and previous studies involving these types of materials suggest using "Topology Optimization Method" (TOM) to enhance the performance of piezoelectric transducers by distributing piezoelectric material over fixed isotropic and orthotropic substrate or to optimize the fiber orientation of orthotropic materials with piezoelectric patches previously established. Thus, this thesis proposes the development of a methodology based on the TOM to design laminated piezocomposite shell transducers by considering simultaneously the optimization of distribution and the polarization direction of the piezoelectric material, and also the optimization of the fiber orientation orthotropic material, which is free to assume different values along the same composite layer. By using this methodology, numerical results are obtained for actuators and sensors under static response, and energy harvesting devices with an electrical circuit coupled, in dynamic damped analysis. In the case of sensors and energy harvesting devices, which are subjected to mechanical loads, the mechanical stresses in the structure are also considered, which must satisfy two stress criteria to prevent failure: von Mises for isotropic materials and Tsai-Wu for orthotropic materials.

Keywords: Topology Optimization, Piezoelectric Transducers, Piezocomposite Shell, Fiber Orientation, Energy Harvesting, Mechanical Stress Constraints. 


\section{Conteúdo}

\section{Lista de Figuras}

\section{Lista de Tabelas}

\section{Lista de Abreviaturas}

1 INTRODUÇÃO 1

1.1 Otimização Topológica aplicada a Transdutores Piezocompósitos . . . . . . 2

1.2 Restrição de Falha Mecânica em Problemas de Otimização Topológica (OT) 5

1.3 Procedimento do MOT . . . . . . . . . . . . . . . . . . . . . 6

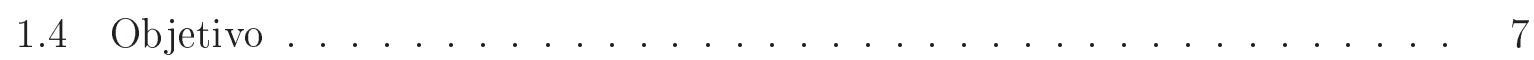

1.5 Justificativa . . . . . . . . . . . . . . . . . . . . 8

1.6 Contribuição Científica . . . . . . . . . . . . . . . . . . . . . . . 9

1.7 Organização do Trabalho . . . . . . . . . . . . . . . . . . . . . 9

2 MODELAGEM DE CASCAS PIEZOCOMPÓSITAS UTILIZANDO O MÉTODO DE ELEMENTOS FINITOS 11

2.1 Equações Piezelétricas . . . . . . . . . . . . . . . . . . . . . . . 12

2.2 Geometria e Cinemática do Elemento de Casca . . . . . . . . . . . . 15

2.2.1 Definição dos Sistemas de Coordenadas . . . . . . . . . . . . . 15

2.2.2 Obtenção das Coordenadas Nodais . . . . . . . . . . . . . 16

2.2.3 Campo de Deslocamentos . . . . . . . . . . . . . 17

2.2.4 Campo de Deformações . . . . . . . . . . . . . . . 18

2.3 Potencial Elétrico . . . . . . . . . . . . . . . . . . . . . . . . . . . . . 22 
2.4 Campo Elétrico . . . . . . . . . . . . . . . . . . . . . . . . 23

2.5 Tensões Mecânicas . . . . . . . . . . . . . . . . . . . . . . 24

2.6 Equações de Equilíbrio . . . . . . . . . . . . . . . . . . . . . . . 25

2.7 Teoria "Layer-Wise" para Acoplamento entre Camadas . . . . . . . . . . . 27

3 MÉTODO DE OTIMIZAÇÃO TOPOLÓGICA 30

3.1 Introdução ao MOT . . . . . . . . . . . . . . . . . . . 30

3.2 Formulação Adotada . . . . . . . . . . . . . . . . . . . . . . . . . . 32

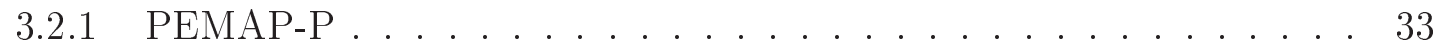

3.2.2 Otimização de Orientação de Fibras utilizando o Método OMD . . 35

3.2.3 Variáveis de Projeto Nodais e Técnica de Projeção . . . . . . . . . 37

3.3 Otimização Topológica com Restrição de Falha Mecânica . . . . . . . . . . 41

3.3.1 Relaxação das Tensões . . . . . . . . . . . . . . . . . . . . . . 44

3.3.2 Critérios de von Mises e Tsai-Wu . . . . . . . . . . . . . . . 45

3.3.3 Restrição Global de Tensão . . . . . . . . . . . . . . . . . . . . . 46

3.4 Implementação do MOT . . . . . . . . . . . . . . . . . . . . . . . . . . 49

3.5 Análise de Sensibilidades das Formulações do MOT . . . . . . . . . . . . . 49

3.5.1 Derivadas das Matrizes de Rigidez e Massa . . . . . . . . . . . . . 50

3.5.2 Derivada da Restrição de Falha Mecânica . . . . . . . . . . . . . . 52

4 PROJETO ESTÁTICO DE ATUADORES PIEZOCOMPÓSITOS DE CASCA $\quad 55$

4.1 Histórico . . . . . . . . . . . . . . . . . . . . . 56

4.2 Excitação por Potencial Elétrico . . . . . . . . . . . . . . 56

4.3 Formulação do Problema de OT para Atuadores no Domínio Contínuo . . 57

4.4 Formulação do Problema de OT para Atuadores no Domínio Discretizado . 59

4.5 Análise de Sensibilidades . . . . . . . . . . . . . . . . . . 60

4.6 Resultados . . . . . . . . . . . . . . . . . . . . 62 


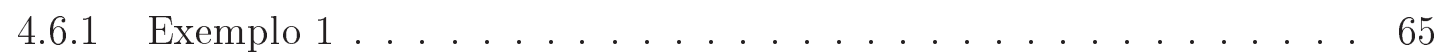

4.6 .2 Exemplo 2 . . . . . . . . . . . . . . . . . . 74

4.7 Discussão . . . . . . . . . . . . . . . . . . . . . 82

5 PROJETO ESTÁTICO DE SENSORES PIEZOCOMPÓSITOS DE CASCA COM RESTRIÇÃO DE TENSÃO

5.1 Modelagem dos Eletrodos no Método de Elementos Finitos (MEF) Piezelétrico de Casca . . . . . . . . . . . . . . . . . . . . . . 85

5.2 Formulação do Problema de OT para Sensores no Domínio Contínuo . . . 87

5.3 Formulação do Problema de OT para Sensores no Domínio Discretizado . . 88

5.4 Análise de Sensibilidades . . . . . . . . . . . . . . . . . . . . . 89

5.5 Resultados . . . . . . . . . . . . . . . . . . . . 90

5.6 Exemplo $1 \ldots \ldots \ldots \ldots \ldots$. . . . . . . . . . . . . . . . 91

5.7 Exemplo $2 \ldots \ldots \ldots \ldots$. . . . . . . . . . . . . . . . . . . . . . .

5.8 Discussão . . . . . . . . . . . . . . . . . . . . . . 107

6 PROJETO DINÂMICO DE COLETORES DE ENERGIA PIEZOCOMPÓSITOS DE CASCA COM RESTRIÇÃO DE TENSÃO 109

6.1 Excitação por Deslocamento Mecânico e Modelagem dos Eletrodos . . . . . 111

6.2 Formulação do Problema de OT para Coletores de Energia no Domínio Contínuo . . . . . . . . . . . . . . . . . . . . 112

6.3 Formulação do Problema de OT para Coletores de Energia no Domínio Discretizado . . . . . . . . . . . . . . . . . . . . . . . . 114

6.4 Análise de Sensibilidades . . . . . . . . . . . . . . . . . . . . 115

6.5 Resultados . . . . . . . . . . . . . . . . . . . 116

6.6 Exemplo $1 \ldots \ldots \ldots \ldots$. . . . . . . . . . . . . . . . . . . . . . .

6.7 Exemplo $2 \ldots \ldots \ldots \ldots \ldots \ldots$

6.8 Discussão . . . . . . . . . . . . . . . . . . . . . . . . 135 
7.1 Sugestão de Trabalhos Futuros . . . . . . . . . . . . . . . . . 137

Referências

A.1 Definição do Sistema de Coordenadas Nodal-Local . . . . . . . . . . . . . . 149

A.2 Obtenção da matriz elástica $\mathbf{C}$ para materiais isotrópicos e ortotrópicos . . 150

\section{Apêndice B - Validação do MEF Implementado}

B.1 Atuador Bilaminar Plano . . . . . . . . . . . . . . . . . 153

B.2 Sensor de Placa Piezelétrico . . . . . . . . . . . . . . . . . . 154

B.3 Atuador de Casca Piezocompósito . . . . . . . . . . . . . . . 155

B.4 Coletor de Energia em Regime Harmônico com Circuito Elétrico Acoplado 157

B.5 Travamento por Cisalhamento . . . . . . . . . . . . . 160

B.6 Conclusão . . . . . . . . . . . . . . . . . . . . . . . 162

Apêndice C - Propriedade dos Materiais $\quad 163$

Apêndice D - Método de Otimização Topológica $\quad 164$

D.1 Introdução . . . . . . . . . . . . . . . . . . . . . . . . 164

D.2 Conceitos do MOT . . . . . . . . . . . . . . . 166

D.2.1 Domínio Fixo Estendido . . . . . . . . . . . . . 166

D.2.2 Modelo de Material . . . . . . . . . . . . . . . . . . . 167

D.2.3 Aspectos Numéricos do MOT . . . . . . . . . . . . . 168 


\section{Lista de Figuras}

1.1 Estrutura de casca laminada piezocompósita. . . . . . . . . . . . . . 2

1.2 Representação de atuadores do tipo bilaminar (a) e "C-block" (b). . . . . . 3

1.3 Procedimento de projeto de um transdutor piezocompósito de casca utilizando o MOT abordado nessa tese. . . . . . . . . . . . . . . 7

2.1 Representação do elemento de casca degenerado obtido a partir de um elemento sólido 3D, com a posição dos nós na superfície de referência, e do elemento isoparamétrico de 8 nós. . . . . . . . . . . . . . . . . 12

2.2 Representação das hipóteses cinemática (a) e mecânica (b) em coordenadas genéricas $(1,2,3) \ldots \ldots \ldots \ldots \ldots \ldots \ldots$

2.3 Sistema de coordenada para um elemento de casca piezelétrico de 8 nós. . . 15

2.4 Sistema de coordenada do material. Direção principal da fibra do material ortotrópico representada pela linha tracejada. . . . . . . . . . . . . . 16

2.5 Representação da transformação entre sistemas de coordenadas: (a) global para local; (b) local para material. . . . . . . . . . . . . . . . . 20

2.6 Variação do potencial elétrico ao longo da espessura: (a) linear e (b)

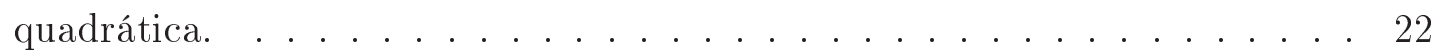

2.7 Representação de uma estrutura piezocompósita de casca. . . . . . . . . . . 27

2.8 Deformações ao longo da espessura de cascas compostas. . . . . . . . . . . 28

2.9 Adesão entre duas camadas $m$ e $n \ldots \ldots \ldots \ldots \ldots$

3.1 Modelo de microestrutura para o método da homogeneização. ...... . 31

3.2 Método OMD para otimização de ângulo, onde $\theta_{i}$ é o ângulo candidato, $\vartheta_{i}$ e $\mathbf{c}_{\theta_{i}}^{E}$ são, respectivamente, a variável de orientação e a matriz constitutiva associada ao ângulo $i$, e $n^{c}$ é o número total de ângulos candidatos. . . . . . 37

3.3 Representação da técnica de projeção. . . . . . . . . . . . . . . . . . . . 39 
3.4 Função "Heaviside" para vários valores de $\beta_{p} \ldots \ldots$. . . . . . . . . . . . . 39

3.5 Representação genérica de um domínio de projeto que apresenta um ótimo singular. . . . . . . . . . . . . . . . . . . 42

3.6 Distribuição de tensão ao longo da espessura em uma estrutura sujeita à flexão

3.7 Fluxograma do procedimento de otimização implementado. . . . . . . . . . 50

4.1 Representação do domínio de projeto de um atuador piezocompósito. Os contornos tracejados representam os deslocamentos $\hat{\mathbf{U}}_{1}$ devido à aplicação de potencial elétrico $\Delta \phi_{1}$.

4.2 Representação do empilhamento de camadas para o exemplo de atuadores de placa. A mesma sequência é utilizada para o exemplo de casca. As cores azul, vermelho e roxo, indicam as camadas de material piezelétrico, de alumínio e de fibra de carbono, respectivamente. . . . . . . . . . . .

4.3 Esquema dos eletrodos para aplicação de potencial elétrico. . . . . . . . . . 64

4.4 Domínio de projeto para o exemplo 1 de atuador. Medidas em mm. . . . . 66

4.5 Forma deformada da estrutura para o caso 0 do atuador de casca, ou seja, sem otimização, com as camadas piezelétricas totalmente cheias e com polarização negativa.

4.6 Resultado da otimização de topologia das camadas piezelétricas para o caso 1 de atuadores de casca.

4.7 Resultado da otimização de polarização das camadas piezelétricas para o caso 1 de atuadores de casca.

4.8 Forma deformada da estrutura para o caso 1 de atuadores de casca. Em vermelho está representada a camada de alumínio, e em azul e verde está representada a camada piezelétrica com polarização positiva e negativa, respectivamente.

4.9 Distribuição das tensões de von Mises das camadas de material piezelétrico para o caso 1 de atuadores de casca. Valores de tensão em MPa, onde o valor máximo da escala representa o valor máximo de tensão nas camadas piezelétricas. . . . . . . . . . . . . . . . . . .

4.10 Resultado da otimização de topologia para o caso 2 de atuadores de casca. 
4.11 Resultado da otimização de polarização para o caso 2 de atuadores de casca. 70

4.12 Resultado da otimização de orientação de fibras e a forma deformada da estrutura para o caso 2 de atuadores de casca. . . . . . . . . . . . . . . . 70

4.13 Distribuição das tensões de von Mises das camadas de material piezelétrico para o caso 2 de atuadores de casca. Valores de tensão em MPa, onde o valor máximo da escala representa o valor máximo de tensão nas camadas piezelétricas. . . . . . . . . . . . . . . . . . 71

4.14 Resultado da otimização de topologia para o caso 3 de atuadores de casca. 72

4.15 Resultado da otimização de polarização para o caso 3 de atuadores de casca. 72

4.16 Resultado da otimização de orientação de fibras e a forma deformada da estrutura para o caso 3 de atuadores de casca. . . . . . . . . . . . . . . . 73

4.17 Distribuição das tensões de von Mises das camadas de material piezelétrico para o caso 3 de atuadores de casca. Valores de tensão em MPa, onde o valor máximo da escala representa o valor máximo de tensão na camada piezelétrica. .......................... 74

4.18 Domínio de projeto para atuadores. Medidas em mm. . . . . . . . . . . . 74

4.19 Forma deformada da estrutura para o caso 0 de atuador de placa, ou seja, sem otimização, com as camadas piezelétricas totalmente cheias e com polarização negativa.

4.20 Resultado da otimização de topologia das camadas piezelétricas para o caso 1 de atuadores de placa. . . . . . . . . . . . . . . . . . . 76

4.21 Resultado da otimização de polarização das camadas piezelétricas para o caso 1 de atuadores de placa. . . . . . . . . . . . . . . . 76

4.22 Forma deformada da estrutura para o caso 1 de atuadores de placa. Em vermelho está representada a camada de alumínio, e em verde está representada a camada piezelétrica com polarização negativa. . . . . . . . . 76

4.23 Distribuição das tensões de von Mises das superfícies superior e inferior das camadas de material piezelétrico para o caso 1 de atuadores de placa. Valores de tensão em MPa, onde os valores máximos da escala representam os limites de tensão. . . . . . . . . . . . . . . . . . . . . . . 77

4.24 Resultado da otimização de topologia para o caso 2 de atuadores de placa. 78 
4.25 Resultado da otimização de polarização para o caso 2 de atuadores de placa. 78

4.26 Resultado da otimização de orientação de fibras e a forma deformada da estrutura para o caso 2 de atuadores de placa. . . . . . . . . . . . . . . 78

4.27 Distribuição das tensões de von Mises das camadas de material piezelétrico para o caso 2 de atuadores de placa. Valores de tensão em MPa, onde o valor máximo da escala representam o valor máximo de tensão nas camadas piezelétricas. . . . . . . . . . . . . . . . . . 79

4.28 Resultado da otimização de topologia para o caso 3 de atuadores de placa . 80

4.29 Resultado da otimização de polarização para o caso 3 de atuadores de placa. 80

4.30 Resultado da otimização de orientação de fibras e a forma deformada da estrutura para o caso 3 de atuadores de placa. . . . . . . . . . . . . . . . 81

4.31 Distribuição das tensões de von Mises das camadas de material piezelétrico para o caso 3 de atuadores de placa. Valores de tensão em MPa, onde o valor máximo da escala representa o valor máximo de tensão nas camadas piezelétricas. .

5.1 Representação do domínio de projeto de um sensor piezocompósito. A aplicação de $\mathbf{f}_{2}$ gera os deslocamentos $\mathbf{U}_{2}$ e o potencial elétrico $\Delta \phi_{2} \ldots$. . . 8

5.2 Domínio de projeto para o exemplo de sensor de casca piezocompósito. Camada interna é o substrato (alumínio ou alumínio/fibra de carbono) e a camada externa é de material piezelétrico.

5.3 Forma deformada da estrutura para o caso 0 do projeto de sensores de casca, ou seja, sem otimização, com as camadas piezelétricas totalmente cheias e com polarização negativa. . . . . . . . . . . . . . . . . . . 93

5.4 Distribuição das tensões de von Mises para o caso 0 do projeto de sensores de casca. Valores máximos: 85,16 MPa e 49,30 MPa para as camadas de alumínio e piezelétrica, respectivamente. . . . . . . . . . . . . . . . . 94

5.5 Resultado da otimização de topologia (a) e de polarização (b) da camada de material piezelétrico para o caso 1 do projeto de sensores de casca. . . . 95

5.6 Forma deformada da estrutura para o caso 1 do projeto de sensores de casca. 95 
5.7 Distribuição das tensões de von Mises para o caso 1 do projeto de sensores de casca. Valores máximos: 91,77 MPa e 57,08 MPa para as camadas de alumínio e piezelétrica, respectivamente. . . . . . . . . . . . . . . 96

5.8 Resultado da otimização de topologia (a) e de polarização (b) da camada de material piezelétrico para o caso 2 do projeto de sensores de casca. . . . 97

5.9 Forma deformada da estrutura para o caso 2 do projeto de sensores de casca. 97

5.10 Distribuição das tensões de von Mises para o caso 2 do projeto de sensores de casca. Valores máximos: 92,89 MPa e 48,69 MPa para as camadas de alumínio e piezelétrica, respectivamente. . . . . . . . . . . . . . . 98

5.11 Resultado da otimização de topologia (a) e de polarização (b) da camada de material piezelétrico para o caso 4 do projeto de sensores de casca. . . .

5.12 Resultado da otimização de orientação de fibras (a) e forma deformada (b) do resultado pós-processado para o caso 4 do projeto de sensores de casca. Em vermelho está representada a camada de alumínio, e em azul e verde está representada a camada piezelétrica com polarização positiva e negativa, respectivamente. . . . . . . . . . . . . . . . 10

5.13 Distribuição das tensões de Tsai-Wu da camada de fibra de carbono e de von Mises das camadas de alumínio e de material piezelétrico para o caso 4 do exemplo 1 de coletores de energia. Valores de tensão de von Mises em MPa. . . . . . . . . . . . . . . . . . . . . . 101

5.14 Domínio de projeto para o exemplo de sensor de placa piezocompósito. Camada inferior é o substrato (alumínio ou alumínio/fibra de carbono) e a camada superior é de material piezelétrico. . . . . . . . . . . . . . . . . . . 102

5.15 Forma deformada da estrutura para o caso 0 do projeto de sensores de placa, ou seja, sem otimização, com as camadas piezelétricas totalmente cheias e com polarização negativa. . . . . . . . . . . . . . . . . . . . 103

5.16 Distribuição das tensões de von Mises para o caso 0 do projeto de sensores de placa. Valores em MPa. . . . . . . . . . . . . . . . . . 103

5.17 Resultado da otimização de topologia (a) e de polarização (b) da camada de material piezelétrico para o caso 1 do projeto de sensores de placa. . . . 104 
5.18 Forma deformada da estrutura para o caso 1 do projeto de sensores de placa. A camada de alumínio está representada na cor vermelha e a camada piezelétrica está representada nas cores azul e verde, com polarização positiva e negativa, respectivamente. . . . . . . . . . . . . . . . . . . 104

5.19 Distribuição das tensões de von Mises para o caso 1 do projeto de sensores de placa. Valores em MPa. . . . . . . . . . . . . . . . . . . 105

5.20 Resultado da otimização de topologia (a) e de polarização (b) da camada de material piezelétrico para o caso 2 do projeto de sensores de placa. . . . 106

5.21 Forma deformada da estrutura para o caso 2 do projeto de sensores de placa. A camada de fibra de carbono está representada na cor roxa e a camada piezelétrica está representada nas cores azul e verde, com polarização positiva e negativa, respectivamente. . . . . . . . . . . . . . 106

5.22 Distribuição das tensões de Tsai-Wu da camada de fibra de carbono e de von Mises das camadas de alumínio e de material piezelétrico para o caso 2 de coletores de energia de placa. Valores de tensão de von Mises em MPa. 107

6.1 Representação de uma estrutura com excitação por movimento de uma base oscilante. . . . . . . . . . . . . . . . . . . . . . 111

6.2 Representação do domínio de projeto de um coletor de energia piezocompósito. . . . . . . . . . . . . . . . . . . . . 113

6.3 Domínio de projeto para coletores de energia de placa. Medidas em mm. . 119

6.4 Forma deformada da estrutura para o caso 0 de coletores de energia de placa, ou seja, sem otimização, com a camada piezelétrica totalmente cheia e polarização positiva. . . . . . . . . . . . . . . . . . . . 120

6.5 Distribuição das tensões de von Mises das superfícies superior e inferior das camadas de material piezelétrico para o caso 0 de coletores de energia de placa. Valores de tensão em MPa, onde os valores máximos da escala representam os limites de tensão. . . . . . . . . . . . . . . . . . . 120

6.6 Resultado da otimização de topologia (a) e de polarização (b) das camadas piezelétricas para o caso 1 de coletores de energia de placa. . . . . . . . . . 121 
6.7 Forma deformada do resultado pós-processado para o caso 1 de coletores de energia de placa. Em vermelho está representada a camada de alumínio, e em azul e verde está representada a camada piezelétrica com polarização positiva e negativa, respectivamente. . . . . . . . . . . . . . . . . 121

6.8 Distribuição das tensões de von Mises das superfícies superior e inferior das camadas de material piezelétrico para o caso 1 de coletores de energia de placa. Valores de tensão em $\mathrm{MPa}$, onde os valores máximos da escala representam os limites de tensão. . . . . . . . . . . . . . . . . . . . . . 122

6.9 Resultado da otimização de topologia (a) e de polarização (b) das camadas piezelétricas para o caso 2 de coletores de energia de placa. . . . . . . . . . 123

6.10 Resultado da otimização de orientação de fibras (a) e forma deformada (b) do resultado pós-processado para o caso 2 de coletores de energia de placa. Em vermelho está representada a camada de alumínio, e em azul e verde está representada a camada piezelétrica com polarização positiva e negativa, respectivamente. . . . . . . . . . . . . . . . 123

6.11 Distribuição das tensões de Tsai-Wu da camada de fibra de carbono e de von Mises das camadas de alumínio e de material piezelétrico para o caso 2 de coletores de energia de placa. Valores de tensão de von Mises em MPa. 124

6.12 Gráfico logarítmico da resposta em frequência dos três casos apresentados anteriormente. . . . . . . . . . . . . . . . . . 125

6.13 Domínio curvo de meio cilindro para o exemplo 2 do projeto de coletores de energia. Medidas em mm. . . . . . . . . . . . . . . . . . . . 127

6.14 Forma deformada da estrutura para o caso 0 de coletores de energia de casca, ou seja, sem otimização, com a camada piezelétrica totalmente cheia e polarização positiva. . . . . . . . . . . . . . . . . . 128

6.15 Duas vistas da distribuição das tensões de von Mises das superfícies críticas das camadas de alumínio e de material piezelétrico para o caso 0 de coletores de energia de casca. Valores de tensão em MPa, onde os valores máximos da escala representam os limites de tensão. . . . . . . . . . . . . . . . . . . 129

6.16 Resultado da otimização de topologia (a) e de polarização (b) das camadas piezelétricas para o caso 1 de coletores de energia de casca, representados em duas vistas. . . . . . . . . . . . . . . . . . . . . . . . 130 
6.17 Forma deformada do resultado pós-processado para o caso 1 de coletores de energia de casca. Em vermelho está representada a camada de alumínio, e em azul e verde está representada a camada piezelétrica com polarização positiva e negativa, respectivamente.

6.18 Duas vistas da distribuição das tensões de von Mises das superfícies críticas das camadas de material piezelétrico para o caso 1 de coletores de energia de casca. Valores de tensão em MPa, onde os valores máximos da escala representam os limites de tensão. . . . . . . . . . . . . . . . . . . . 131

6.19 Resultado da otimização de topologia (a) e de polarização (b) das camadas piezelétricas para o caso 2 de coletores de energia de casca, representados em duas vistas. . . . . . . . . . . . . . . . . . . . . . . . 132

6.20 Resultado da otimização de orientação de fibras para o caso 2 de coletores de energia de casca. . . . . . . . . . . . . . . . . . . . 133

6.21 Forma deformada do resultado pós-processado para o caso 2 de coletores de energia de casca. Em vermelho está representada a camada de alumínio, e em azul e verde está representada a camada piezelétrica com polarização positiva e negativa, respectivamente.

6.22 Duas vistas da distribuição das tensões de von Mises da superfície crítica das camadas de material piezelétrico para o caso 2 de coletores de energia de casca. Valores de tensão em MPa, onde os valores máximos da escala representam os limites de tensão.

6.23 Gráfico logarítmico da resposta em frequência dos três casos apresentados anteriormente. . . . . . . . . . . . . . . . . . 135

7.1 Materiais piezelétricos fibrosos, ou MFC. . . . . . . . . . . . . 138

B.1 Modelo da viga bilaminar piezelétrica. . . . . . . . . . . . . . . 153

B.2 Modelo de um sensor de placa piezelétrico. . . . . . . . . . . . . . . . . . 154

B.3 Modelo de um atuador de casca piezocompósito. . . . . . . . . . . . . 156

B.4 Deslocamento radial normalizado $w / h$ ao longo da linha média em $z=l / e$ da primeira camada. . . . . . . . . . . . . . . . . 156

B.5 Modelo utilizado para verificação dos coletores de energia. . . . . . . . . . 158

B.6 Gráfico da resposta em frequência do modelo apresentado entre 0 e $2000 \mathrm{~Hz} .158$ 
B.7 Gráfico logarítmico da potência elétrica em função da frequência do modelo apresentado entre 0 e $2000 \mathrm{~Hz}$. . . . . . . . . . . . . . . . . . . . 159

B.8 Gráfico da resposta em frequência do modelo apresentado entre 400 e 700 $\mathrm{Hz}$ com intervalo de $1 \mathrm{~Hz}$. . . . . . . . . . . . . . . . . . . . 160

B.9 Gráfico da resposta em frequência do modelo apresentado entre 1200 e 1500 $\mathrm{Hz}$ com intervalo de $1 \mathrm{~Hz}$. . . . . . . . . . . . . . . . . . . . . 160

B.10 Modelo utilizado para verificação do efeito de "shear locking". . . . . . . . . . 161

D.1 Exemplo de 3 tipos de otimização estrutural. . . . . . . . . . . . . . . . . 165

D.2 Conceito de Domínio Fixo Estendido. . . . . . . . . . . . . . . . . 166

D.3 Representação de um resultado com escalas de cinza e o padrão de tabuleiro.168

D.4 Representação de um exemplo onde a solução depende do refinamento da malha. . . . . . . . . . . . . . . . . . . . . . . 169 


\section{Lista de Tabelas}

2.1 Definição das matrizes e vetores eletroelásticos. . . . . . . . . . . . 26

4.1 Dados dos resultados apresentados para o exemplo de atuadores de casca. . 73

4.2 Dados dos resultados apresentados para o exemplo de atuadores de placa. . 82

5.1 Dados dos resultados apresentados para o exemplo de sensores de casca. . . 100

5.2 Dados dos resultados apresentados para o exemplo de sensores de casca. . . 107

6.1 Dados dos resultados apresentados para o exemplo de coletores de energia de casca. . . . . . . . . . . . . . . . . . . . . 125

6.2 Dados dos resultados apresentados para o exemplo de coletores de energia de casca. . . . . . . . . . . . . . . . . . . . . . 132

A.1 Coordenadas naturais $r$ e $s$ para os nós de um elemento. . . . . . . . . . 149

B.1 Deslocamento vertical no ponto A. . . . . . . . . . . . . . . 154

B.2 Potenciais elétricos $\Delta \phi_{1}$ a $\Delta \phi_{4}$ [V] e deslocamento $u_{z}^{A}[\mathrm{~mm}] . \ldots . . . .155$

B.3 Efeito do travamento para finas camadas de material piezelétrico. . . . . . 162

C.1 Propriedades do Alumínio . . . . . . . . . . . . . . . . 163

C.2 Propriedades do Aço . . . . . . . . . . . . . . . . . . 163

C.3 Propriedades do material piezelétrico utilizado por Kögl e Bucalem (2005a). 163

C.4 Propriedades do material piezelétrico utilizado por Balamurugan e Narayanan (2008). . . . . . . . . . . . . . . . . 163

C.5 Propriedades da fibra de carbono com matriz de epóxi (Performance Composite Ltd, ). . . . . . . . . . . . . . . . . . 163 


\section{Lista de Abreviaturas}

MEF Método de Elementos Finitos

MOT Método de Otimização Topológica

OT Otimização Topológica

OMD Otimização de Material Discreto

DMO "Discrete Material Optimization"

SIMP "Simple Isotropic Material with Penalization"

PEMAP-P "Piezoelectric Material with Penalization and Polarization"

MMA "Method of Moving Asymptotes"

MFC "Macro Fiber Composite"

TO "Topology Optimization"

TOM "Topology Optimization Method"

ESO "Evolutionary Structural Optimization"

3D Tridimensional

DFE Domínio Fixo Estendido 


\section{INTRODUÇÃO}

Materiais piezelétricos têm a propriedade de converter energia elétrica em energia mecânica, ou vice-e-versa. Essa propriedade tem sido alvo de uma grande variedade de trabalhos nas últimas décadas, desde aplicações de ultrassom como, sonares (DESILETS et al., 1999; SHUYU, 2005), levitação acústica (ANDRADE; BUIOCHI; ADAMOWSKI, 2010; XIE et al., 2006), diagnóstico e tratamento médico (WHITTINGHAM, 2007; HAAR, 2007), micro e nano posicionadores e manipuladores (CARBONARI; SILVA; NISHIWAKI, 2005; CARBONARI, 2008), sensores de pressão (NAKASONE; SILVA, 2010) e acústicos (LI; CHENG, 2010), e até mais recentemente dispositivos de coleta de energia (SODANO; INMAN, 2004; BEEBY; TUDOR; WHITE, 2006; ERTURK; INMAN, 2008; RUPP et al., 2009; RUPP; DUNN; MAUTE, 2010). Geralmente, os materiais piezelétricos são utilizados juntamente com outros tipos de materiais, como por exemplo, materiais isotrópicos e ortotrópicos elásticos ${ }^{1}$. Estruturas compostas por esses tipos de materiais são chamados de estruturas piezocompósitas. Neste trabalho, são analisadas estruturas piezocompósitas finas, onde a espessura é muito menor que o comprimento e largura. Elas são denominadas de estruturas de placa e casca multi-camadas piezocompósita e têm sido alvo de grande interesse na área da Engenharia, principalmente no campo de estruturas inteligentes, onde podem ser utilizadas para sensoriamento, atuação e coleta de energia (TZOU; TSENG, 1990; AGRAWAL; TREANOR, 1999; MUKHERJEE; JOSHI, 2002; SODANO; INMAN; PARK, 2005; PARK et al., 2010b).

Um exemplo de estrutura de casca multi-camadas piezocompósita é mostrado na Fig. 1.1, onde camadas de diferentes tipos de material são empilhadas de modo que se possa aproveitar as vantagens oferecidas por cada material. O material isotrópico pode servir como "molde" para que se possa colar as outras camadas, como um espaçador entre camadas diferentes, ou até mesmo como eletrodo para as camadas de material piezelétrico. O material ortotrópico pode ser utilizado tanto para aumentar quanto para reduzir a rigidez da estrutura, dependendo dos requisitos do projeto, modificando-se o

\footnotetext{
${ }^{1}$ Denomina-se, nessa tese, materiais ortotrópicos elásticos aqueles compostos por materiais fibrosos, como fibra de vidro ou carbono, dentro de uma matriz de epóxi. A partir deste ponto, esses materiais serão denominados somente de materiais ortotrópicos, por simplicidade.
} 


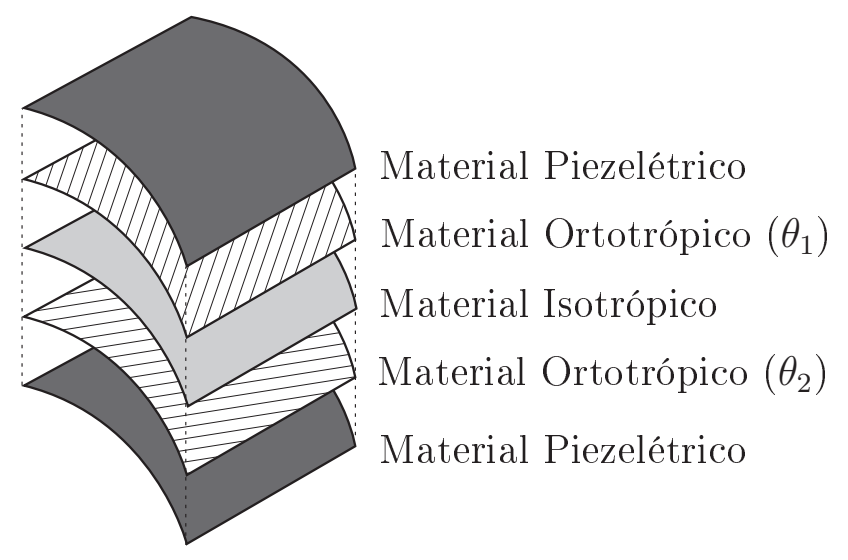

Figura 1.1: Estrutura de casca laminada piezocompósita.

ângulo de orientação das fibras em cada camada. Já as camadas de material piezelétrico servirão como mecanismos de atuação ou sensoriamento, utilizando tanto a aplicação de potencial elétrico quanto deformações mecânicas, respectivamente, como princípio de funcionamento. As aplicações para esse tipo de estrutura incluem o controle de deformação (por sensoriamento e atuação) em, por exemplo, asas de aeronaves (TZOU; TSENG, 1990) e pás de turbinas (LUND, 2009), espelhos deformáveis para telescopia espacial (RIVA et al., 2010), antenas refletoras (AGRAWAL; TREANOR, 1999), sensores de monitoramento de tráfego (LI; YANG; LI, 2006) e também atuadores como os bilaminares e de casca cilíndrica (também conhecidos como "C-block") (BREI; MOSKALIK, 1997; ERVIN; BREI, 1998; BALAMURUGAN; NARAYANAN, 2001) (ver Fig. 1.2), que são amplamente empregados na indústria de automação e também na aeronáutica (MUKHERJEE; JOSHI, 2002).

\subsection{Otimização Topológica aplicada a Transdutores Piezocompósitos}

A distribuição de material piezelétrico afeta o desempenho da estrutura, e portanto, a quantidade, forma, tamanho, localização e sentido de polarização devem ser simultaneamente considerados num problema de otimização. Nesse sentido, ferramentas de otimização, como o Método de Otimização Topológica (MOT), têm sido amplamente utilizadas para encontrar a topologia ótima de transdutores piezocompósitos de casca (KÖGL; SILVA, 2005; KANG; TONG, 2008a, 2008b; ZHENG; CHANG; GEA, 2009; RUPP et al., 2009; RUPP; DUNN; MAUTE, 2010). O MOT é uma ferramenta de projeto estrutural que tem como princípio básico encontrar a distribuição ótima de material dentro de um domínio fixo pré-determinado para maximizar ou minimizar funções objetivos e satisfazer 


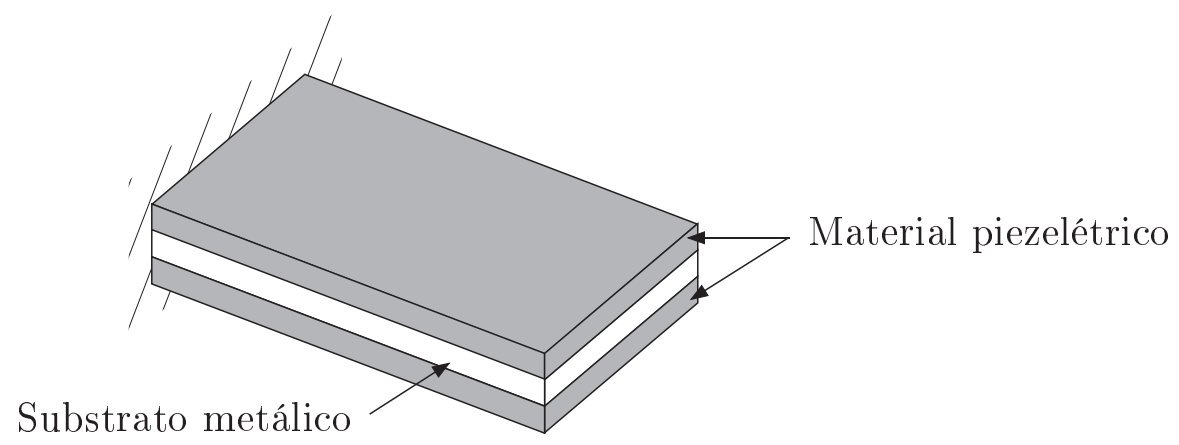

(a) Atuador bilaminar

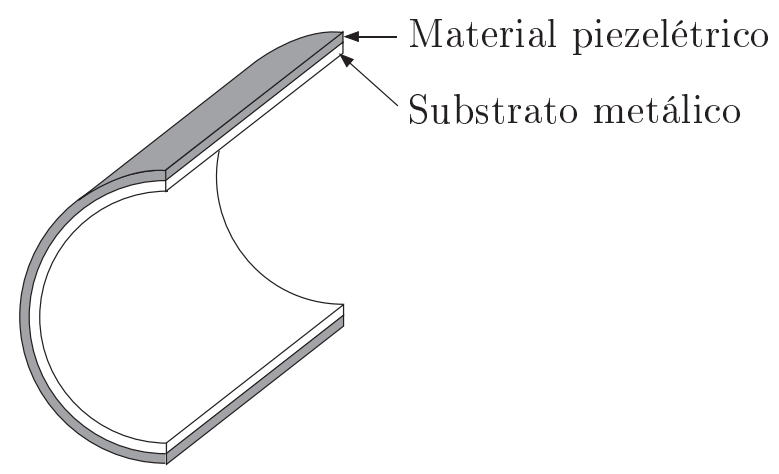

(b) Atuador "C-block"

Figura 1.2: Representação de atuadores do tipo bilaminar (a) e "C-block" (b).

certos requisitos de projeto.

Um dos trabalhos pioneiros na aplicação do MOT no projeto de transdutores piezelétricos foi Silva, Fonseca e Kikuchi (1997), que empregam o MOT para o projeto de microestruturas (ou células unitárias) de materiais piezelétricos, buscando melhorar o desempenho desses materiais. Seguindo esse contexto, a aplicação do MOT no projeto de atuadores piezelétricos flextensionais é apresentada por Silva e co-autores (SILVA et al., 1999; SILVA; KIKUCHI, 1999b, 1999a; SILVA; NISHIWAKI; KIKUCHI, 1999, 2000), utilizando o conceito de energia mútua e de flexibilidade média (NISHIWAKI et al., 1998; FRECKER et al., 1997). Utilizando um software de MOT pode-se obter o projeto de um atuador piezelétrico flextensional apenas especificando o domínio de projeto, a excitação de entrada (potencial ou carga elétrica) e a direção dos deslocamentos de saída em um determinado ponto desejado. Isso viabilizou o projeto de atuadores piezelétricos não-convencionais como garras, grampos, pinças, etc (CARBONARI, 2008).

Além da maximização de deslocamentos e tensões elétricas, outras características de transdutores piezelétricos também podem ser aperfeiçoados através do MOT. Silva e 
Kikuchi (1999b) empregam o MOT para o projeto de transdutores piezelétricos buscando o aumento dos coeficientes de acoplamento eletromecânico e abordando também requisitos referentes à frequência de ressonância desses transdutores. Carbonari, Silva e Nishiwaki (2005) empregam o MOT para o projeto de micromanipuladores piezelétricos para manipulação e posicionamento de precisão.

A otimização da distribuição de material piezelétrico, juntamente com a otimização do sentido de polarização em transdutores de placas e casca, foi inicialmente apresentada por Kögl e Silva (2005) que propuseram um novo modelo de material, o "Piezoelectric Material with Penalization and Polarization" (PEMAP-P). Esse modelo se baseia no modelo tradicional "Simple Isotropic Material with Penalization" (SIMP) para modelar a distribuição de material piezelétrico e sugere a criação de uma nova variável de projeto para modelar o sentido de polarização do material piezelétrico.

Recentemente, vários trabalhos têm sido publicados para otimizar transdutores piezocompósitos de placas e cascas. Kang e Tong (2008a) apresentam um trabalho aplicando o MOT em placas laminadas piezelétricas, numa abordagem estática, através da distribuição de material piezelétrico e também da determinação dos valores de tensão elétrica ótima para acionamento do atuador. Em seguida, revendo a viabilidade prática de aplicar a distribuição ótima de tensões elétricas obtidas, Kang e Tong (2008b) apresentam novos resultados, desta vez considerando uma distribuição de tensão elétrica uniforme em todo o domínio. Mais recentemente, Howard et al. (2009) utilizam o MOT para estruturas de placas e cascas piezelétricas, apresentando resultados para o projeto das deformações livres em transdutores piezelétricos finos.

Considerando o projeto de coletores de energia baseados em estruturas multicamadas de placas e cascas, Zheng, Chang e Gea (2009) aplicaram otimização topológica para maximizar a conversão de energia mecânica em elétrica operando no modo estático. Além disso, Rupp et al. (2009), Rupp, Dunn e Maute (2010) consideraram o projeto dinâmico de coletores de energia e otimizaram a topologia das camadas piezelétricas acopladas à uma camada de material isotrópico, com um circuito elétrico externo de coleta de energia acoplado à estrutura.

Essencialmente, esses trabalhos consideram apenas materiais isotrópicos como substrato e aplicam a otimização topológica nas camadas piezelétricas, ou otimizam o substrato tendo a posição do material piezelétrico pré-determinado. Existem na literatura vários trabalhos de otimização topológica em estruturas mecânicas de placas e cascas, que propõem a utilização de materiais ortotrópicos para aumentar a rigidez da estrutura 
mediante esforços mecânicos (DUVAUT et al., 2000; STEGMANN; LUND, 2005a; LUND, 2009; LINDGAARD; LUND, 2011). Materiais ortotrópicos possuem propriedades elásticas que, em geral, são diferentes ao longo de cada eixo. Um exemplo bem comum de material ortotrópico é um polímero reforçado por fibras paralelas de vidro ou carbono. A rigidez de tal material compósito é geralmente maior na direção paralela às fibras do que nas direções transversais. Utilizando essa propriedade, as fibras em cada camada de material ortotrópico podem ser orientadas arbitrariamente de modo a, por exemplo, maximizar a rigidez da estrutura.

Seguindo essa abordagem, alguns trabalhos (RAY; REDDY, 2004b, 2004a, 2005; WANG; TAI; QUEK, 2006) propuseram a utilização de materiais fibrosos em transdutores piezelétricos e aplicaram a otimização topológica para melhorar o desempenho em aplicações de controle de vibração e de forma. Contudo, esses trabalhos utilizam o MOT ou para otimizar a distribuição de material piezelétrico mantendo as fibras das camadas ortotrópicas constantes, ou otimizando a orientação das fibras dessas camadas sendo que a posição do material piezelétrico é pré-estabelecido e fixo durante o processo de otimização.

\subsection{Restrição de Falha Mecânica em Problemas de OT}

Outro tópico importante abordado nesta tese é a inclusão de restrições de tensão mecânica no problema de otimização. Estruturas sujeitas a esforços mecânicos externos apresentam uma distribuição de tensão mecânica que depende da amplitude da força aplicada, da frequência utilizada (em casos dinâmicos), da distribuição de material no domínio, e, no caso de se utilizar materiais ortotrópicos, depende também da orientação das fibras desse material.

$\mathrm{Na}$ área de transdutores piezocompósitos, essa abordagem tem grande relevância em aplicações de sensores e coletores de energia, uma vez que eles estão sujeitos a carregamentos mecânicos externos, que muitas vezes são dados de projeto e não podem ser alterados. Por exemplo, no caso de coletores de energia, para aproveitar a energia de vibração de uma estrutura, o transdutor deve ser acoplado à essa estrutura e utilizar a mesma amplitude e frequência da vibração. Já no caso de sensores de pressão, a estrutura deve suportar grandes pressões, por exemplo. Porém, no caso de atuadores, a análise de tensões mecânicas não é necessária pois a própria estrutura piezelétrica provoca os deslocamentos, os quais podem ser controlados pela amplitude da tensão elétrica aplicada.

É possível encontrar na literatura, diversos trabalhos que consideram a tensão 
mecânica em suas formulações de otimização topológica, tanto como restrição (que é mais comumente utilizado) (CHENG; GUO, 1997; DUYSINX; BENDSØE, 1998; LE et al., 2010) ou como função objetivo (YANG; CHEN, 1996; ALlAIRE; JOUVE; MAILLOT, 2004; GROENWOLD; HAFTKA, 2006). Entretanto, esses trabalhos estão limitados a problemas estruturais como, por exemplo, minimização de volume com restrição de falha mecânica, ou a minimização da tensão mecânica máxima com restrição de volume de material. $\mathrm{Na}$ área de estruturas piezocompósitas de casca, nenhum trabalho envolvendo tensões mecânicas foi encontrado até o momento da finalização desta tese. Assim, esta tese propõe uma nova abordagem no projeto de transdutores piezocompósitos incluindo restrição de falha mecânica na formulação de OT, sendo que, para cada tipo de material, é utilizado um diferente critério para calcular a restrição. Nas camadas de material isotrópico a restrição é utilizada para garantir que as deformações permaneçam no regime linear elástico, ou seja, as tensões mecânicas devem ser menores que a tensão mecânica de escoamento do material (LE et al., 2010). Nas camadas de material piezelétrico, a restrição evita a despolarização da cerâmica (CALDERON-MORENO, 2001), sendo que a tensão mecânica que causa a despolarização é muito menor que a tensão mecânica de ruptura da cerâmica. Finalmente, nas camadas de material ortotrópico, a restrição é utilizada para evitar a falha da estrutura (GROENWOLD; HAFTKA, 2006), ou seja, a ruptura das fibras ou da matriz de epóxi.

\subsection{Procedimento do MOT}

O procedimento típico de projeto de uma estrutura de casca utilizando o MOT é apresentado na Fig. 1.3, que divide-se em seis etapas: definição do domínio inicial, discretização em elementos finitos, obtenção configuração ótima da estrutura (considerando a topologia, polarização e orientação das fibras), pós-processamento do resultado, verificação através de uma nova análise de MEF e, finalmente, a fabricação do protótipo para verificação experimental, completando o ciclo de projeto.

Neste trabalho é apresentado o procedimento de OT até a verificação do MEF do resultado pós-processado, ou seja, este trabalho não trata a fabricação nem os procedimentos que a fabricação abrange. 


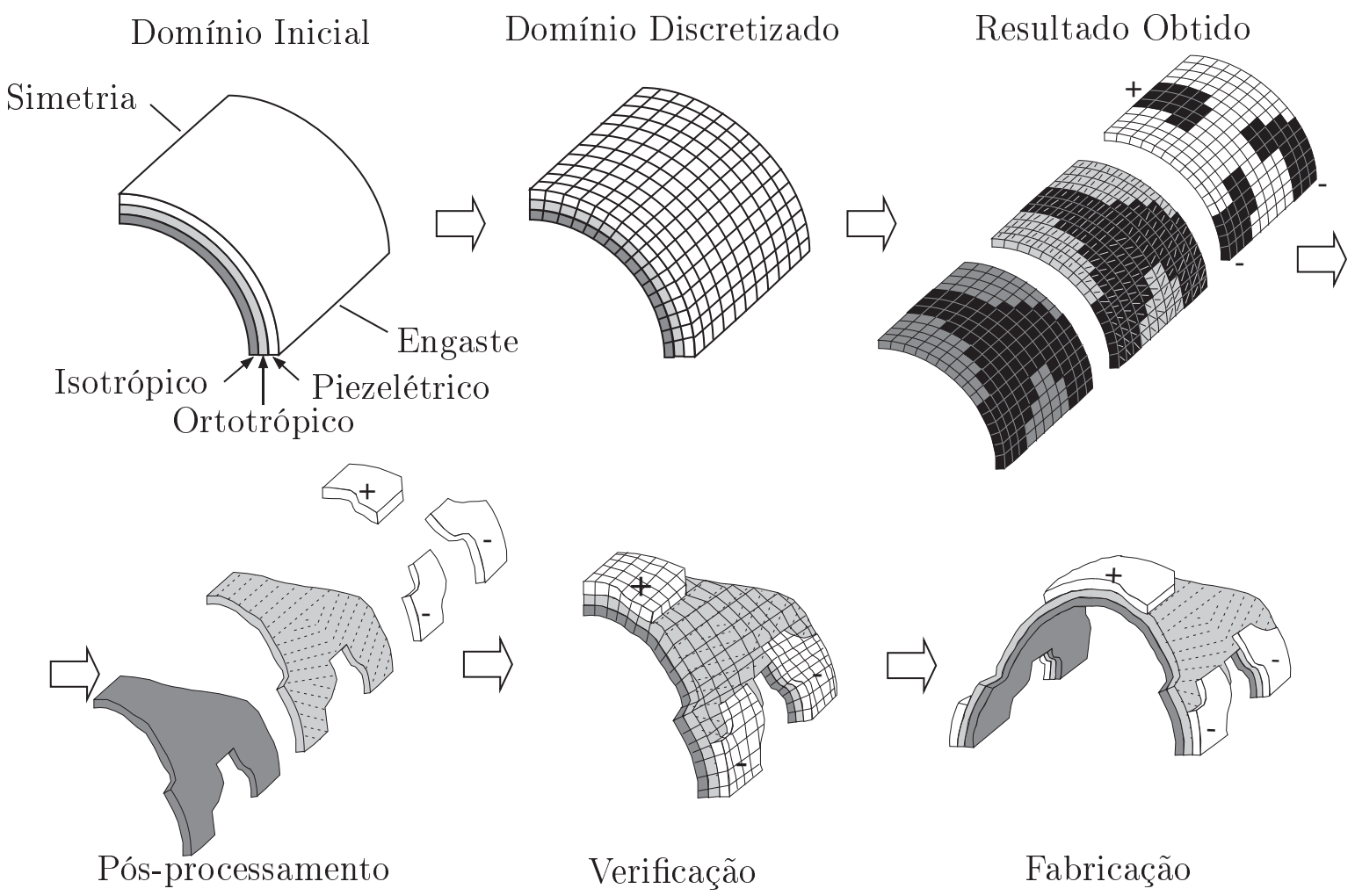

Figura 1.3: Procedimento de projeto de um transdutor piezocompósito de casca utilizando o MOT abordado nessa tese.

\subsection{Objetivo}

O objetivo deste trabalho é desenvolver uma metodologia para projetar transdutores piezocompósitos multicamadas de casca utilizando métodos de otimização topológica. A otimização visa melhorar o desempenho desses transdutores através da distribuição de material (piezelétrico e não-piezelétrico), da escolha do sentido de polarização do material piezelétrico e da escolha da orientação das fibras do material ortotrópico, numa otimização simultânea desses parâmetros. Como mencionado anteriormente, restrições de tensão mecânica são incluídas na formulação para evitar falhas estruturais que podem vir a ocorrer caso as deformações ultrapassem um certo limite.

São considerados três tipos de projetos diferentes: atuadores, sensores e coletores de energia. No projeto de atuadores piezocompósitos, deseja-se maximizar os deslocamentos em certos pontos de uma dada estrutura, num regime estático. Para isso, propõe-se maximizar os deslocamentos em pontos pré estabelecidos nas direções desejadas, gerados pela aplicação de potencial elétrico nos eletrodos das camadas piezelétricas. No projeto de sensores, o objetivo é melhorar a sensibilidade elétrica da estrutura dada uma perturbação mecânica conhecida, também no regime estático, respeitando certos limites de tensão mecânica. Assim, propõe-se maximizar o potencial elétrico gerado nos eletrodos da 
camada piezelétrica devido ao carregamento mecânico aplicado, mantendo o valor máximo de tensão mecânica abaixo de uma dada restrição. Finalmente, no caso dos coletores de energia, dada uma vibração externa conhecida, deseja-se maximizar a potência elétrica gerada pela estrutura ligada à um resistor externo, também sujeita a limitações de tensão mecânica.

\subsection{Justificativa}

Nas últimas décadas, o crescente aumento da utilização de materiais compósitos na engenharia tem despertado grande interesse na área de estruturas inteligentes. A otimização da distribuição de material piezelétrico já tem sido apresentada em diversos trabalhos (KÖGL; SILVA, 2005; KANG; TONG, 2008a, 2008b; ZHENG; CHANG; GEA, 2009; RUPP et al., 2009; RUPP; DUNN; MAUTE, 2010). Entretanto, a possibilidade de modificar a rigidez da estrutura do substrato (estrutura de suporte do material piezelétrico) alterando-se a orientação das fibras do material compósito, traz consigo novos conceitos na otimização estrutural de transdutores piezelétricos. No caso de atuadores, orientando-se apropriadamente as fibras do material compósito pode-se ter um substrato mais flexível numa dada direção de atuação, e mais rígido em outras direções, melhorando-se assim o controle de deslocamentos e também a distribuição de tensões mecânicas. Essa mesma abordagem pode ser aplicada para sensores e coletores de energia, onde é possível melhorar a resposta elétrica do transdutor otimizando, conjuntamente, a distribuição e a polarização de material piezelétrico e a orientação das fibras.

Além disso, como dito anteriormente, a distribuição de tensão mecânica na estrutura é um fator que pode invalidar a solução ótima visto que, no caso de sensores e coletores de energia, a amplitude e frequência do carregamento mecânico pode não ser controlável pelo projetista. Portanto, é necessário incluir restrições de tensão mecânica no problema de otimização.

É possível encontrar na literatura trabalhos que consideram a otimização da distribuição de material piezelétrico (que também utilizam materiais ortotrópicos) e outros trabalhos que consideram a otimização da orientação das fibras utilizando material piezelétrico em locais pré-determinados que permanecem fixos durante a otimização. Contudo, não foi encontrado nenhum trabalho considerando, simultaneamente, a otimização de distribuição de material piezelétrico, bem como sua polarização, e também a otimização de orientação das fibras do material ortotrópico, e nem trabalhos que considerem restrição de falha mecânica no projeto de transdutores piezocompósitos. 
Assim, este trabalho traz um estudo pioneiro no projeto otimizado de transdutores piezocompósitos laminados de casca.

\subsection{Contribuição Científica}

Como principal contribuição científica deste trabalho destaca-se o desenvolvimento de uma metodologia que combina métodos de otimização topológica considerando, simultaneamente, a distribuição e sentido de polarização do material piezelétrico e a orientação das fibras do material ortotrópico. É proposto então combinar um modelo de material para a otimização de distribuição e polarização do material piezelétrico (PEMAP-P) junto com uma técnica de otimização de material discreto para otimizar a orientação das fibras do material ortotrópico. A generalidade da metodologia implementada permite projetar diferentes tipos de transdutores, como atuadores, sensores e coletores de energia de forma totalmente sistemática. De forma a divulgar essa nova metodologia de projeto um artigo foi publicado em periódico internacional (KIYONO; SILVA; REDDY, 2012) e mais dois foram submetidos (MELLO et al., Submetido em julho de 2012; KIYONO et al., ). Além disso, outros dois artigos foram apresentados em congressos internacionais (KIYONO; NAKASONE; SILVA, 2010; KIYONO; SILVA, 2011).

Outra contribuição deste trabalho é a inclusão de restrições de tensão mecânica na formulação do problema de otimização de transdutores piezocompósitos de casca. As restrições de tensão são necessárias para garantir que os limites de falha dos materiais não sejam ultrapassados. São apresentados resultados numéricos para comprovar que as restrições de tensão são necessárias. Essa abordagem é nova e não foram encontrados trabalhos na literatura com essas características.

Além dessas contribuições, o elemento de casca piezelétrico apresentado neste trabalho tem como contribuição, não a sua formulação, que pode ser encontrada em diversos trabalhos (BATHE, 1996; KÖGL; SILVA, 2005; BALAMURUGAN; NARAYANAN, 2008), mas sim na compilação das informações e no detalhamento da implementação apresentada (que inclui a integração do MEF com o MOT) para futuras pesquisas.

\subsection{Organização do Trabalho}

No Capítulo 2 é apresentada a formulação detalhada do elemento de casca laminada piezelétrica. 
Em seguida no Capítulo 3 é feito um detalhamento do MOT utilizado, incluindo o modelo de material, modelo de otimização de orientação de fibras e a técnica de projeção "Heaviside", e a formulação da restrição de falha mecânica proposta nesta tese. Neste capítulo, é apresentado também a análise de sensibilidades dessas formulações.

Nos Capítulos 4, 5 e 6 são apresentados os problemas de otimização e os projetos otimizados de atuadores, sensores e coletores de energia, respectivamente, considerando estruturas piezocompósitas de casca. Dentro de cada um desses capítulos, é apresentada uma introdução sobre o tema, a formulação do problema de otimização topológica, análise de sensibilidades e resultados referente a cada tipo de projeto.

Finalmente no Capítulo 7 é apresentada a conclusão geral do trabalho realizado e, adicionalmente, são apresentadas também algumas sugestões de trabalhos futuros que podem ser realizados a partir deste.

No Apêndice A, é descrita a obtenção do sistema de coordenadas nodal-local e da matriz elástica anisotrópica, para serem utilizados no MEF. No Apêndice B, a validação do MEF é apresentado em 5 casos: para atuadores planos somente com material piezelétrico, para sensor de placa com alumínio e com material piezelétrico, para atuador piezocompósito de casca, com fibra de carbono e material piezelétrico, para coletores de energia em regime dinâmico, e para travamento por cisalhamento. 


\section{MODELAGEM DE CASCAS PIEZOCOMPÓSITAS UTILIZANDO O MÉTODO DE ELEMENTOS FINITOS}

Cada iteração da rotina de otimização topológica exige que análises estruturais de geometrias complexas sejam realizadas, o que é feito utilizando o MEF. O elemento de casca piezelétrica utilizado neste estudo é baseado na teoria de casca laminada de primeira ordem (também conhecido como elemento de casca degenerado) (AHMAD; IRONS; ZIENKIEWICZ, 1970; REDDY, 2004) e no elemento piezelétrico de placas e cascas proposto por Kögl e Bucalem (2005b).

O elemento de casca degenerado foi inicialmente proposto por Ahmad, Irons e Zienkiewicz (1970), onde as equações elásticas 3D de um elemento sólido tridimensional são discretizadas em relação à sua superfície média. O elemento de casca degenerado é muito atraente pois pode ser empregado para análise de estruturas de placas e cascas compósitas laminadas com qualquer geometria, espessas ou delgadas e podendo ser utilizada para análises linear e não-linear. Na Fig. 2.1(a) encontra-se a representação desse tipo de elemento, com a posição dos nós na superfície média, e na Fig. 2.1(b) pode ser vista a representação de um elemento isoparamétrico de 8 nós, com o sistema de coordenadas naturais, normalizadas nas 3 direções.

O comportamento de casca degenerada é simulado pela imposição de hipóteses cinemáticas e mecânicas, as quais correspondem a uma generalização da teoria de placas de Reissner-Mindlin:

- A hipótese cinemática: Partículas inicialmente alinhadas com um vetor diretor $V_{n}^{k}$, inicialmente normal à superfície média, permanecem alinhadas com este vetor durante todo o movimento. Entretanto, o vetor diretor $V_{n}^{k}$ não necessariamente deve permanecer normal à superfície média durante o movimento. A Fig. 2.2(a) 


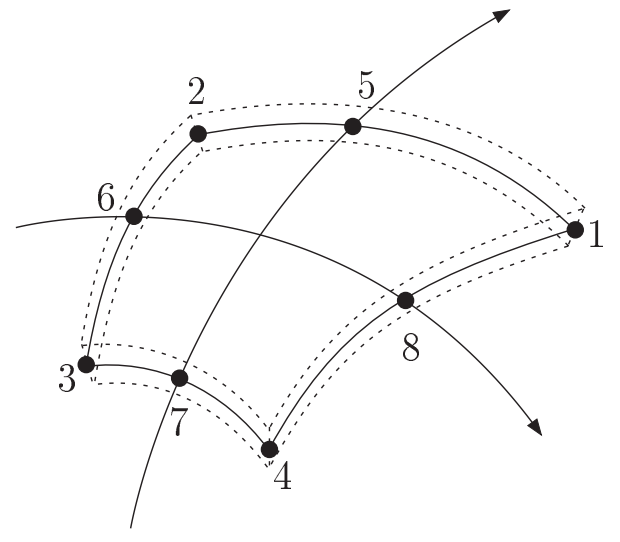

(a)

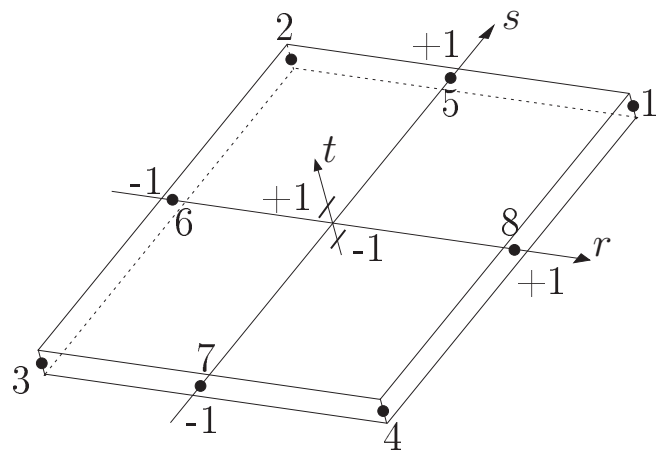

(b)

Figura 2.1: Representação do elemento de casca degenerado obtido a partir de um elemento sólido 3D, com a posição dos nós na superfície de referência, e do elemento isoparamétrico de 8 nós.

ilustra esta hipótese com as posições inicial e a deformada.

- A hipótese mecânica: A componente normal do tensor das tensões de Cauchy na direção do vetor diretor de um ponto qualquer da superfície média deve ser nula durante todo o movimento, admitindo assim um estado plano de tensão. Na Fig. 2.2(b) são apresentadas as tensões de Cauchy num sistema de coordenadas genérico $(1,2,3)$, considerando $\sigma_{33}=0$.

Dessa forma, o elemento utilizado neste trabalho possui 8 nós (um em cada vértice e um no centro de cada aresta), considera tanto as deformações por flexão, quanto por cisalhamento (BATHE, 1996; KÖGL; SILVA, 2005; BALAMURUGAN; NARAYANAN, 2008), operando no regime linear elástico. Apesar de ser um elemento custoso computacionalmente, pois possui muitos graus de liberdade, este elemento apresenta uma boa relação entre eficiência, precisão e baixa complexidade de implementação para tratar problemas como travamento por cisalhamento ou de membrana.

\subsection{Equações Piezelétricas}

As equações constitutivas piezelétricas descrevem o acoplamento entre os campos elétrico e elástico da seguinte maneira (IKEDA, 1990):

$$
\begin{aligned}
\boldsymbol{\sigma} & =\mathbf{c}^{E} \boldsymbol{\varepsilon}-\mathbf{e E} \\
\mathbf{D} & =\mathbf{e}^{T} \boldsymbol{\varepsilon}+\boldsymbol{\epsilon}^{S} \mathbf{E}
\end{aligned}
$$




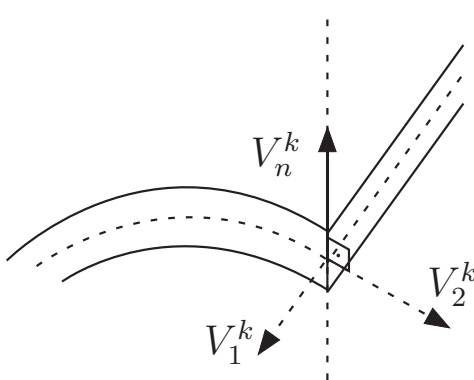

Posição Inicial

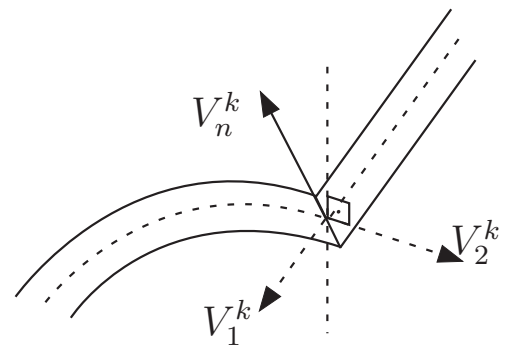

Posição Deformada

(a) Hipótese Cinemática
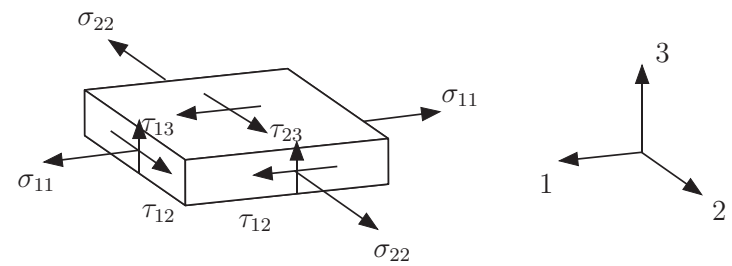

(b) Hipótese Mecânica

Figura 2.2: Representação das hipóteses cinemática (a) e mecânica (b) em coordenadas genéricas $(1,2,3)$.

onde $\boldsymbol{\sigma}, \boldsymbol{\varepsilon}, \mathbf{D}$ e $\mathbf{E}$ são os vetores de tensão mecânica, deformação mecânica, deslocamento elétrico e campo elétrico, respectivamente, sendo que

$$
\begin{gathered}
\boldsymbol{\sigma}=\left\{\begin{array}{llllll}
\sigma_{11} & \sigma_{22} & \sigma_{33} & \tau_{12} & \tau_{23} & \tau_{13}
\end{array}\right\}^{T} \\
\boldsymbol{\varepsilon}=\left\{\begin{array}{llllll}
\varepsilon_{11} & \varepsilon_{22} & \varepsilon_{33} & \gamma_{12} & \gamma_{23} & \gamma_{13}
\end{array}\right\}^{T} \\
\mathbf{D}=\left\{\begin{array}{llll}
D_{1} & D_{2} & D_{3}
\end{array}\right\}^{T} \\
\mathbf{E}=\left\{\begin{array}{llll}
E_{1} & E_{2} & E_{3}
\end{array}\right\}^{T}
\end{gathered}
$$

As propriedades do material são descritas pelo tensor elástico $\mathbf{c}^{E}$ (obtido em campo elétrico constante), tensor piezelétrico e e o tensor dielétrico $\boldsymbol{\epsilon}^{S}$ (obtido em deformação mecânica constante). Neste trabalho, utilizam-se materiais piezelétricos e puramente elásticos. Os materiais piezelétricos mais comumente utilizados para atuadores e sensores são da classe de simetria hexagonal das famílias 6mm (PZT-5A) e $\infty m$ (PZT-5H), respectivamente, cuja polarização é definida na direção 3. Para esses tipos de materiais 
piezelétricos, as matrizes $\mathbf{c}^{E}$, e e $\boldsymbol{\epsilon}^{S}$ podem ser escritas da seguinte forma:

$$
\mathbf{c}^{E}=\left[\begin{array}{cccccc}
c_{11} & c_{12} & c_{13} & 0 & 0 & 0 \\
c_{12} & c_{22} & c_{23} & 0 & 0 & 0 \\
c_{13} & c_{23} & c_{33} & 0 & 0 & 0 \\
0 & 0 & 0 & c_{66} & 0 & 0 \\
0 & 0 & 0 & 0 & c_{44} & 0 \\
0 & 0 & 0 & 0 & 0 & c_{55}
\end{array}\right] ; \mathbf{e}=\left[\begin{array}{ccc}
0 & 0 & e_{31} \\
0 & 0 & e_{32} \\
0 & 0 & e_{33} \\
0 & 0 & 0 \\
0 & e_{24} & 0 \\
e_{15} & 0 & 0
\end{array}\right] ; \boldsymbol{\epsilon}^{S}=\left[\begin{array}{ccc}
\epsilon_{11} & 0 & 0 \\
0 & \epsilon_{22} & 0 \\
0 & 0 & \epsilon_{33}
\end{array}\right]
$$

onde $c_{22}=c_{11}, c_{23}=c_{13}, c_{55}=c_{44}, c_{66}=\left(c_{11}-c_{12}\right) / 2, e_{32}=e_{31}, e_{24}=e_{15}$ e $\epsilon_{22}=\epsilon_{11}$.

Para materiais puramente elásticos, utiliza-se neste trabalho os materiais isotrópicos e ortotrópicos, sendo que as constantes piezelétricas e dielétricas devem ser nulas nesses casos. Para ambos os tipos de materiais, a matriz elástica $\mathbf{c}^{E}$ pode ser obtida a partir dos módulos de Young e dos coeficientes de Poisson, cujo cálculo pode ser visto no Apêndice A.2.

No caso da modelagem por elementos de casca, de acordo com a hipótese mecânica, assume-se o estado plano de tensões $\sigma_{33}=0$ (AHMAD; IRONS; ZIENKIEWICZ, 1970), o que possibilita escrever as equações constitutivas piezelétricas, de uma forma condensada, pelo seguinte sistema matricial:

$$
\left\{\begin{array}{c}
\sigma_{11} \\
\sigma_{22} \\
\tau_{12} \\
\tau_{23} \\
\tau_{13} \\
\hline D_{1} \\
D_{2} \\
D_{3}
\end{array}\right\}=\left[\begin{array}{ccccc|ccc}
c_{11}^{*} & c_{12}^{*} & 0 & 0 & 0 & 0 & 0 & e_{31}^{*} \\
c_{12}^{*} & c_{11}^{*} & 0 & 0 & 0 & 0 & 0 & e_{31}^{*} \\
0 & 0 & c_{66} & 0 & 0 & 0 & 0 & 0 \\
0 & 0 & 0 & k s * c_{44} & 0 & 0 & e_{15} & 0 \\
0 & 0 & 0 & 0 & k s * c_{44} & e_{15} & 0 & 0 \\
\hline 0 & 0 & 0 & 0 & e_{15} & -\epsilon_{11} & 0 & 0 \\
0 & 0 & 0 & e_{15} & 0 & 0 & -\epsilon_{11} & 0 \\
e_{31}^{*} & e_{31}^{*} & 0 & 0 & 0 & 0 & 0 & -\epsilon_{33}^{*}
\end{array}\right]\left\{\begin{array}{c}
\varepsilon_{11} \\
\varepsilon_{22} \\
\gamma_{12} \\
\gamma_{23} \\
\gamma_{13} \\
\hline-E_{1} \\
-E_{2} \\
-E_{3}
\end{array}\right\}
$$

onde

$$
\begin{array}{lll}
c_{11}^{*}=c_{11}-\frac{\left(c_{13}\right)^{2}}{c_{33}} \quad ; & c_{12}^{*}=c_{12}-\frac{\left(c_{13}\right)^{2}}{c_{33}} \\
e_{31}^{*}=e_{31}-\frac{c_{13}}{c_{33}} e_{33} & ; & \epsilon_{33}^{*}=\epsilon_{33}-\frac{\left(e_{33}\right)^{2}}{c_{33}}
\end{array}
$$




\subsection{Geometria e Cinemática do Elemento de Casca}

Nesta seção, são apresentados quatro conceitos básicos para a formulação do elemento finito de casca: definição dos sistemas de coordenadas, obtenção das coordenadas dos nós, cálculo do campo de deslocamentos e finalmente o cálculo do campo de deformadas.

\subsubsection{Definição dos Sistemas de Coordenadas}

$\mathrm{Na}$ formulação do elemento de casca, deve-se, inicialmente, definir três diferentes sistemas de coordenadas: o sistema global $(x, y, z)$, sistema natural $(r, s, t)$, o sistema local $\left(x^{\prime}, y^{\prime}, z^{\prime}\right)$, como pode ser visto na Fig. 2.3 .

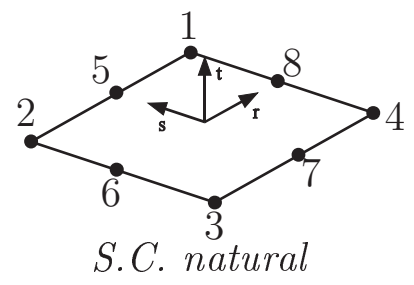

S.C. natural

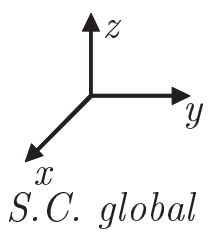

Figura 2.3: Sistema de coordenada para um elemento de casca piezelétrico de 8 nós.

O sistema de coordenada global é definido pelos eixos cartesianos e é adotado para descrever os dados de entrada como coordenadas dos nós e forças aplicadas, bem como resultados de deslocamentos. O sistema de coordenada natural facilita a criação das matrizes de rigidez e massa e dos vetores de força utilizando a integração numérica e pode ser definido a partir da sequência dos nós em cada elemento. O sistema de coordenada local é de extrema importância, pois ele é utilizado para descrever a geometria do elemento e as propriedades do material. Esse sistema é definido, em cada nó, pelos versores unitários $\left\{V_{1}^{k}, V_{2}^{k}, V_{n}^{k}\right\}$ representando $\left(x^{\prime}, y^{\prime}, z^{\prime}\right)$, respectivamente, e o cálculo para obtenção desse sistema é apresentado no Apêndice A.1. A geometria do elemento é representada pela sua curvatura que é definida pela diferença entre os sistemas locais de cada nó, e as propriedades dos materiais devem ser definidas com base nas direções $\left(x^{\prime}, y^{\prime}, z^{\prime}\right)$. Para materiais isotrópicos, nenhuma consideração deve ser tomada, já que as propriedades são iguais para todas as direções. Para materiais piezelétricos, a única consideração importante é definir a direção de polarização comos sendo igual à direção $z^{\prime}$, ao longo da espessura, já que, neste trabalho, são utilizados materiais piezelétricos com isotropia no plano perpendicular ao da polarização. Entretanto, no caso de materiais ortotrópicos, a 
fibra segue uma direção contida no plano perpendicular ao vetor normal da superfície, e o ângulo de orientação da fibra é definido a partir do eixo $x^{\prime}$, aumentando conforme a regra da mão direita ao redor do eixo $z^{\prime}$, como representado na Fig. 2.4.

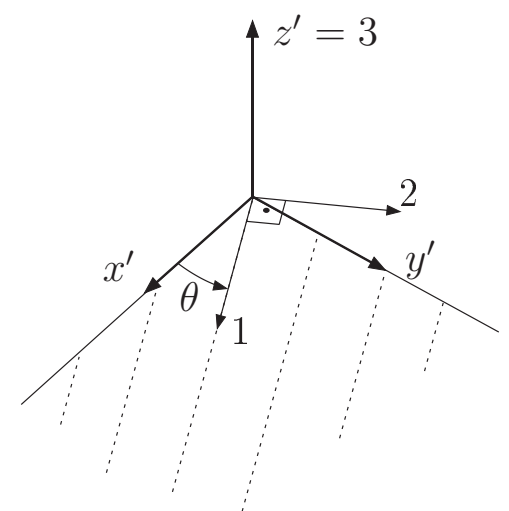

Figura 2.4: Sistema de coordenada do material. Direção principal da fibra do material ortotrópico representada pela linha tracejada.

Assim, é definido mais um sistema de coordenadas chamado sistema de coordenada do material, representado por $(1,2,3)$, mesma notação utilizada nas equações constitutivas ${ }^{1}$ (Eq. (2.8)) para facilitar o entendimento. Esse sistema pode ser adotado por qualquer material, sendo que para materiais isotrópicos e piezelétricos, faz-se $\left(x^{\prime}=1, y^{\prime}=2 \mathrm{e}\right.$ $z^{\prime}=3$ ). Como as propriedades do material são definidas nesse sistema, as tensões e deformações devem ser também obtidas para esse sistema de coordenadas de modo a facilitar os cálculos de critérios de falha, como o critério de Tsai-Wu por exemplo. Ou seja, ao invés de se utilizar as tensões em coordenadas globais $\left(\sigma_{x x}\right.$ e $\left.\sigma_{y y}\right)$, adotam-se as tensões e deformações nas direções longitudinais e transversais $\left(\sigma_{11}\right.$ e $\left.\sigma_{22}\right)$ à direção preferencial das fibras (no caso de material ortotrópico). O cálculo do critério de falha de Tsai-Wu está apresentado mais adiante.

\subsubsection{Obtenção das Coordenadas Nodais}

A cinemática de um elemento de casca de 8 nós é descrita pela interpolação de sua geometria e de suas variáveis de deslocamento. As coordenadas globais de um ponto qualquer do elemento de casca utilizado são dadas por (BATHE, 1996; REDDY, 2004):

$$
\left\{\begin{array}{l}
{ }^{l} x \\
{ }^{l} y \\
{ }^{l} z
\end{array}\right\}=\sum_{k=1}^{8} N_{k}(r, s)\left(\left\{\begin{array}{l}
{ }^{l} x_{k} \\
{ }^{l} y_{k} \\
{ }^{l} z_{k}
\end{array}\right\}+t \frac{h_{k}}{2}\left\{\begin{array}{l}
{ }^{l} V_{n x}^{k} \\
{ }^{l} V_{n y}^{k} \\
{ }^{l} V_{n z}^{k}
\end{array}\right\}\right)
$$

\footnotetext{
${ }^{1}$ As equações constitutivas podem ser utilizadas em qualquer sistema de coordenadas, mediante correta utilização das propriedades do material.
} 
onde $N_{k}(r, s)$ são as funções de forma para os nós $k=1, \ldots, 8$ (BATHE, 1996), $r, s$, e $t$ são as coordenadas isoparamétricas (coordenadas naturais), e:

$$
\begin{aligned}
{ }^{l} x,{ }^{l} y,{ }^{l} z= & \text { vetor que define as coordenadas cartesianas } \\
& \text { de qualquer ponto no elemento; } \\
{ }^{l} x_{k},{ }^{l} y_{k},{ }^{l} z_{k}= & \text { vetor que define as coordenadas nodais; } \\
h_{k}= & \text { espessura do elemento para o nó } k ; \\
{ }^{l} V_{n x}^{k},{ }^{l} V_{n y}^{k},{ }^{l} V_{n z}^{k}= & \text { componentes do vetor diretor }{ }^{l} \mathbf{V}_{n}^{k} \text { para o nó } k ;
\end{aligned}
$$

sendo que todos esse vetores são definidos no sistema global de coordenadas.

O sobrescrito esquerdo $l$ denota a configuração do elemento, podendo ser configuração inicial $l=0$, ou deformada $l=1$.

\subsubsection{Campo de Deslocamentos}

Os deslocamentos de um ponto qualquer do elemento contínuo de casca são obtidos através da diferença entre a coordenada do ponto após e antes da deformação (BATHE, 1996):

$$
\left\{\begin{array}{l}
u \\
v \\
w
\end{array}\right\}=\left\{\begin{array}{c}
{ }^{1} x \\
{ }^{1} y \\
{ }^{1} z
\end{array}\right\}-\left\{\begin{array}{c}
{ }^{0} x \\
{ }^{0} y \\
{ }^{0} z
\end{array}\right\}=\sum_{k=1}^{8} N_{k}(r, s)\left(\left\{\begin{array}{c}
u_{k} \\
v_{k} \\
w_{k}
\end{array}\right\}+t \frac{h_{k}}{2}\left\{\begin{array}{c}
V_{n x}^{k} \\
V_{n y}^{k} \\
V_{n z}^{k}
\end{array}\right\}\right)
$$

onde $\mathbf{V}_{n}^{k}=\left\{\begin{array}{lll}V_{n x}^{k} & V_{n y}^{k} & V_{n z}^{k}\end{array}\right\}^{T}$ representa a diferença entre os vetores diretores antes e depois da deformação:

$$
\mathbf{V}_{n}^{k}={ }^{1} \mathbf{V}_{n}^{k}-{ }^{0} \mathbf{V}_{n}^{k}
$$

Definindo $\alpha_{k}$ e $\beta_{k}$ como as rotações do vetor diretor ${ }^{0} \mathbf{V}_{n}^{k}$ em torno dos vetores ${ }^{0} \mathbf{V}_{1}^{k}$ e ${ }^{0} \mathbf{V}_{2}^{k}$ (que definem o sistema local, ver Fig. 2.2(a)), a expressão Eq. (2.11) pode ser escrita da seguinte maneira:

$$
\mathbf{V}_{n}^{k}=-{ }^{0} \mathbf{V}_{2}^{k} \alpha_{k}+{ }^{0} \mathbf{V}_{1}^{k} \beta_{k}
$$


Substituindo a Eq. (2.12) na Eq. (2.10) chega-se a:

$$
\left\{\begin{array}{l}
u \\
v \\
w
\end{array}\right\}=\sum_{k=1}^{8} N_{k}(r, s)\left(\left\{\begin{array}{l}
u_{k} \\
v_{k} \\
w_{k}
\end{array}\right\}+t \frac{h_{k}}{2}\left[\begin{array}{cc}
-V_{2 x}^{k} & V_{1 x}^{k} \\
-V_{2 y}^{k} & V_{1 y}^{k} \\
-V_{2 z}^{k} & V_{1 z}^{k}
\end{array}\right]\left\{\begin{array}{c}
\alpha_{k} \\
\beta_{k}
\end{array}\right\}\right)
$$

que pode ser escrito como

$$
\mathbf{u}=\left[\begin{array}{llll}
\mathbf{A}_{1} & \mathbf{A}_{2} & \cdots & \mathbf{A}_{k}
\end{array}\right]\left\{\begin{array}{c}
\hat{\mathbf{u}}_{1} \\
\hat{\mathbf{u}}_{2} \\
\vdots \\
\hat{\mathbf{u}}_{k}
\end{array}\right\}=\mathbf{A} \mathbf{u}_{e}
$$

onde

$$
\begin{gathered}
\mathbf{A}_{k}=\left[\begin{array}{ccccc}
N_{k} & 0 & 0 & -t \frac{h_{k}}{2} N_{k} V_{2 x}^{k} & t \frac{h_{k}}{2} N_{k} V_{1 x}^{k} \\
0 & N_{k} & 0 & -t \frac{h_{k}}{2} N_{k} V_{2 y}^{k} & t \frac{h_{k}}{2} N_{k} V_{1 y}^{k} \\
0 & 0 & N_{k} & -t \frac{h_{k}}{2} N_{k} V_{2 z}^{k} & t \frac{h_{k}}{2} N_{k} V_{1 z}^{k}
\end{array}\right] \\
\hat{\mathbf{u}}_{k}=\left\{\begin{array}{lllll}
u_{k} & v_{k} & w_{k} & \alpha_{k} & \beta_{k}
\end{array}\right\}^{T}
\end{gathered}
$$

A obtenção do sistema local (que é um sistema ortonormal) $\left\{{ }^{0} \mathbf{V}_{n}^{k},{ }^{0} \mathbf{V}_{1}^{k},{ }^{0} \mathbf{V}_{2}^{k}\right\}$ está descrita no Apêndice A.1, e sua definição pode ser vista na Fig. 2.2(a).

Assim, define-se o campo de deslocamentos de um elemento genérico de casca composto por 5 graus de liberdade mecânicos por nó, considerando 3 componentes de translação dos nós da superfície média $u_{k}, v_{k}$ e $w_{k}$ (em coordenadas globais), e duas rotações $\alpha_{k}$ e $\beta_{k}$ (em coordenadas locais) do vetor nodal $\mathbf{V}_{n}^{k}$ em relação aos vetores tangentes nodais $\mathbf{V}_{2}^{k}$ e $\mathbf{V}_{1}^{k}$.

\subsubsection{Campo de Deformações}

Uma vez definidos os deslocamentos de um elemento genérico de casca, as componentes do tensor de deformação podem ser obtidas, em coordenadas globais, da seguinte maneira:

$$
\overline{\bar{\varepsilon}}=\left\{\begin{array}{c}
\varepsilon_{x x} \\
\varepsilon_{y y} \\
\varepsilon_{z z} \\
\gamma_{x y} \\
\gamma_{y z} \\
\gamma_{x z}
\end{array}\right\}=\left\{\begin{array}{c}
u,_{x} \\
v,_{y} \\
w,_{z} \\
u,_{y}+v,_{x} \\
v,_{z}+w,_{y} \\
u,_{z}+w,_{x}
\end{array}\right\}=\left[\begin{array}{llll}
\mathbf{B}_{\mathbf{u} 1} & \mathbf{B}_{\mathbf{u} 2} & \cdots & \mathbf{B}_{\mathbf{u} k}
\end{array}\right]\left\{\begin{array}{c}
\hat{\mathbf{u}}_{1} \\
\hat{\mathbf{u}}_{2} \\
\vdots \\
\hat{\mathbf{u}}_{k}
\end{array}\right\}=\mathbf{B}_{\mathbf{u}} \mathbf{u}_{e}
$$


sendo que $\mathbf{B}_{\mathbf{u}}$ é a matriz das derivadas das funções de forma. Cada termo $\mathbf{B}_{\mathbf{u} k}$ é calculado utilizando-se a matriz Jacobiana e as funções de forma, e pode ser escrita como (BATHE, 1996; BALAMURUGAN; NARAYANAN, 2008; REDDY, 2006):

$$
\mathbf{B}_{\mathbf{u} k}=\left[\begin{array}{ccccc}
a_{k} & 0 & 0 & -d_{k} V_{2 x}^{k} & d_{k} V_{1 x}^{k} \\
0 & b_{k} & 0 & -e_{k} V_{2 y}^{k} & e_{k} V_{1 y}^{k} \\
0 & 0 & c_{k} & -g_{k} V_{2 z}^{k} & g_{k} V_{1 z}^{k} \\
b_{k} & a_{k} & 0 & -\left(e_{k} V_{2 x}^{k}+d_{k} V_{2 y}^{k}\right) & \left(e_{k} V_{1 x}^{k}+d_{k} V_{1 y}^{k}\right) \\
0 & c_{k} & b_{k} & -\left(g_{k} V_{2 y}^{k}+e_{k} V_{2 z}^{k}\right) & \left(g_{k} V_{1 y}^{k}+e_{k} V_{1 z}^{k}\right) \\
c_{k} & 0 & a_{k} & -\left(d_{k} V_{2 z}^{k}+g_{k} V_{2 x}^{k}\right) & \left(d_{k} V_{1 z}^{k}+g_{k} V_{1 x}^{k}\right)
\end{array}\right]
$$

onde $k=1,2, \ldots, 8$ para um elemento de casca quadrilátero de 8 nós. Os coeficientes dessa matriz são obtidos por:

$$
\begin{aligned}
& \left\{\begin{array}{l}
a_{k} \\
b_{k} \\
c_{k}
\end{array}\right\}=\left[\begin{array}{ll}
J_{11}^{*} & J_{12}^{*} \\
J_{21}^{*} & J_{22}^{*} \\
J_{31}^{*} & J_{32}^{*}
\end{array}\right]\left\{\begin{array}{l}
N_{k}, r \\
N_{k},{ }_{s}
\end{array}\right\} ; \\
& \left\{\begin{array}{l}
d_{k} \\
e_{k} \\
g_{k}
\end{array}\right\}=\frac{h_{k}}{2}\left(t\left\{\begin{array}{l}
a_{k} \\
b_{k} \\
c_{k}
\end{array}\right\}+\left\{\begin{array}{l}
J_{13}^{*} \\
J_{23}^{*} \\
J_{33}^{*}
\end{array}\right\} N_{k}\right)
\end{aligned}
$$

onde $\mathbf{J}$ é a matrix Jacobiana ( $\mathbf{J}^{*}$ é a sua matriz inversa) de transformação do sistema de coordenadas global $(x, y, z)$ para o sistema de coordenadas natural $(r, s, t)$ (REDDY, 2006):

$$
\mathbf{J}=\left[\begin{array}{lll}
x,_{r} & y, r & z, r \\
x,_{s} & y,_{s} & z,,_{s} \\
x,_{t} & y, t & z, t
\end{array}\right] \quad ; \quad \mathbf{J}^{-1}=\mathbf{J}^{*}=\left[\begin{array}{ccc}
r, x & s,,_{x} & t, x \\
r, y & s,_{y} & t,{ }_{y} \\
r,_{z} & s,,_{z} & t,,_{z}
\end{array}\right]
$$

Como já foi dito, as tensões e deformações devem ser calculadas no sistema de coordenadas do material de modo a facilitar sua interpretação e o cálculo de critérios de falha. Portanto, as deformações definidas anteriormente no sistema de coordenadas globais (Eq. (2.17)) devem ser transformadas em deformações no sistema de coordenadas do material. Para isso, são necessárias duas transformações de coordenadas, a primeira transformando do sistema de coordenadas global para o sistema de coordenadas local, e a segunda transformando o sistema de coordenadas local para o sistema de coordenadas do material. Essas duas transformações podem ser representadas pela Fig. 2.5.

Outro ponto importante a ser considerado vem da hipótese mecânica, mencionada 


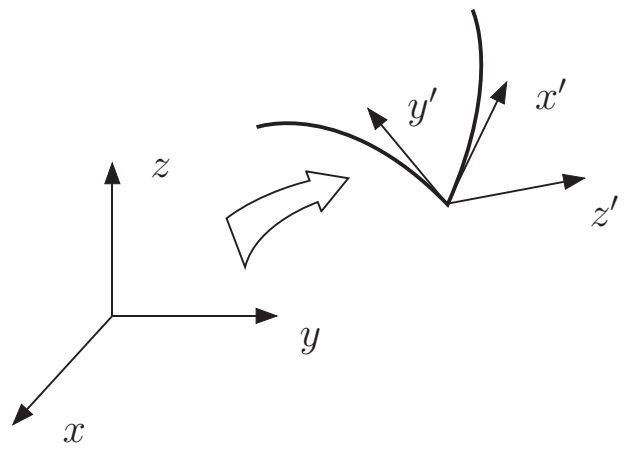

(a)

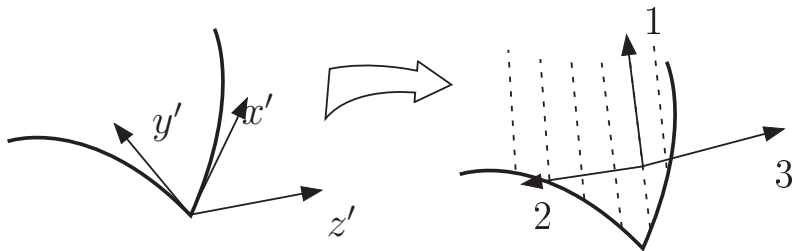

(b)

Figura 2.5: Representação da transformação entre sistemas de coordenadas: (a) global para local; (b) local para material.

anteriormente, que admite o estado plano de tensões no elemento de casca. Assim, tanto no sistema de coordenadas locais, quanto no sistema de coordenadas do material, essa hipótese é válida, ou seja, $\sigma_{z^{\prime} z^{\prime}}=0$ e $\sigma_{33}=0$, e consequentemente $\varepsilon_{z^{\prime} z^{\prime}}$ e $\varepsilon_{33}$ podem ser desconsiderados. Portanto, referente à primeira transformação, as deformações locais $\bar{\varepsilon}$ (no sistema de coordenadas locais) estão relacionadas com as deformações globais $\overline{\bar{\varepsilon}}$ como

$$
\bar{\varepsilon}=\left\{\begin{array}{c}
\varepsilon_{x^{\prime} x^{\prime}} \\
\varepsilon_{y^{\prime} y^{\prime}} \\
\gamma_{x^{\prime} y^{\prime}} \\
\gamma_{y^{\prime} z^{\prime}} \\
\gamma_{x^{\prime} z^{\prime}}
\end{array}\right\}=\mathbf{T}_{\varepsilon}\left\{\begin{array}{c}
\varepsilon_{x x} \\
\varepsilon_{y y} \\
\varepsilon_{z z} \\
\gamma_{x y} \\
\gamma_{y z} \\
\gamma_{x z}
\end{array}\right\}
$$

onde

$$
\mathbf{T}_{\varepsilon}=\left[\begin{array}{cccccc}
l_{1}^{2} & m_{1}^{2} & n_{1}^{2} & l_{1} m_{1} & m_{1} n_{1} & n_{1} l_{1} \\
l_{2}^{2} & m_{2}^{2} & n_{2}^{2} & l_{2} m_{2} & m_{2} n_{2} & n_{2} l_{2} \\
2 l_{1} l_{2} & 2 m_{1} m_{2} & 2 n_{1} n_{2} & l_{1} m_{2}+l_{2} m_{1} & m_{1} n_{2}+m_{2} n_{1} & n_{1} l_{2}+n_{2} l_{1} \\
2 l_{2} l_{3} & 2 m_{2} m_{3} & 2 n_{2} n_{3} & l_{2} m_{3}+l_{3} m_{2} & m_{2} n_{3}+m_{3} n_{2} & n_{2} l_{3}+n_{3} l_{2} \\
2 l_{3} l_{1} & 2 m_{3} m_{1} & 2 n_{3} n_{1} & l_{3} m_{1}+l_{1} m_{3} & m_{3} n_{1}+m_{1} n_{3} & n_{3} l_{1}+n_{1} l_{3}
\end{array}\right]
$$

e $l_{1}, l_{2}, l_{3}, m_{1}, m_{2}, m_{3}, n_{1}, n_{2}$ e $n_{3}$ podem ser convenientemente obtidos a partir da matrix Jacobiana (Eq. (2.20)) de transformação entre o sistema de coordenada global $(x, y, z)$ e 
o natural $(r, s, t)$ :

$$
\begin{aligned}
& \hat{e}_{1}=\left\{\begin{array}{lll}
l_{1} & m_{1} & n_{1}
\end{array}\right\}^{T}=\left(J_{1}\right)_{n o r m} \\
& \hat{e}_{3}=\left\{\begin{array}{lll}
l_{3} & m_{3} & n_{3}
\end{array}\right\}^{T}=\left(J_{1} \times J_{2}\right)_{n o r m} \\
& \hat{e}_{2}=\left\{\begin{array}{lll}
l_{2} & m_{2} & n_{2}
\end{array}\right\}^{T}=\left(\hat{e}_{3} \times \hat{e}_{1}\right)
\end{aligned}
$$

onde $\hat{e}_{1}, \hat{e}_{2}$ e $\hat{e}_{3}$ são os vetores unitários que representam o sistema de coordenadas locais $\left(x^{\prime}, y^{\prime}, z^{\prime}\right)$. $J_{i}$ é a coluna $i$ da matrix Jacobiana, ' $\times$ ' representa o produto cruzado dos vetores e 'norm' indica a norma do vetor.

A segunda transformação leva em conta a orientação das fibras do material ortotrópico, ou seja, é uma rotação no plano da casca (Fig. 2.5(b)), onde as direções $z^{\prime}$ e 3 são iguais. Assim, as deformações no sistema de coordenadas do material $\varepsilon$ se relacionam com as deformações locais $\varepsilon^{\prime}$ da seguinte maneira:

$$
\boldsymbol{\varepsilon}=\left\{\begin{array}{c}
\varepsilon_{11} \\
\varepsilon_{22} \\
\gamma_{12} \\
\gamma_{23} \\
\gamma_{13}
\end{array}\right\}=\mathbf{T}_{\theta}\left\{\begin{array}{c}
\varepsilon_{x^{\prime} x^{\prime}} \\
\varepsilon_{y^{\prime} y^{\prime}} \\
\gamma_{x^{\prime} y^{\prime}} \\
\gamma_{y^{\prime} z^{\prime}} \\
\gamma_{x^{\prime} z^{\prime}}
\end{array}\right\}
$$

onde $\mathbf{T}_{\theta}$ é a matriz de rotação ortotrópica dada por:

$$
\mathbf{T}_{\theta}=\left[\begin{array}{ccccc}
c^{2} & s^{2} & s c & 0 & 0 \\
s^{2} & c^{2} & -s c & 0 & 0 \\
-2 s c & 2 s c & c^{2}-s^{2} & 0 & 0 \\
0 & 0 & 0 & c & -s \\
0 & 0 & 0 & s & c
\end{array}\right]
$$

sendo que $c=\cos (\theta)$ e $s=\operatorname{sen}(\theta)$ e $\theta$ é o ângulo de orientação da fibra (ver Fig. 2.4).

Portanto, as deformações no sistema de coordenadas do material $\varepsilon$ estão relacionadas com deslocamentos globais $\mathbf{u}^{e}$ da seguinte maneira:

$$
\varepsilon=\mathbf{T}_{\theta} \mathbf{T}_{\varepsilon} \mathbf{B}_{u} \mathbf{u}_{e}
$$




\subsection{Potencial Elétrico}

Neste trabalho, o potencial elétrico é interpolado tridimensionalmente dentro do elemento de casca piezelétrico (KÖGL; BUCALEM, 2005b, 2005a; KÖGL; SILVA, 2005; BALAMURUGAN; NARAYANAN, 2008). Considerando que eletrodos são colados nas superfícies inferior e superior da camada piezelétrica, assume-se que essas superfícies são equipotenciais, ou seja, toda a superfície deve possuir o mesmo potencial elétrico. Assim, define-se um grau de liberdade elétrico para representar a ligação entre os eletrodos das superfícies superior e inferior de cada camada piezelétrica. Esse grau de liberdade elétrico é denominado de $\Delta \phi$ e é atribuído no centro de cada elemento. Entretanto, o $\Delta \phi$ de todos os elementos de uma mesma camada piezelétrica são acoplados para simular a existência de eletrodos.

O potencial elétrico pode ser interpolado de maneira linear ou quadrática ao longo da espessura, como representado na Fig. 2.6. Neste trabalho, é utilizada a variação quadrática de potencial elétrico pois assim é possível modelar precisamente o campo elétrico induzido por deformações de flexão e a rigidez resultante da estrutura (KÖGL; BUCALEM, 2005b, 2005a).

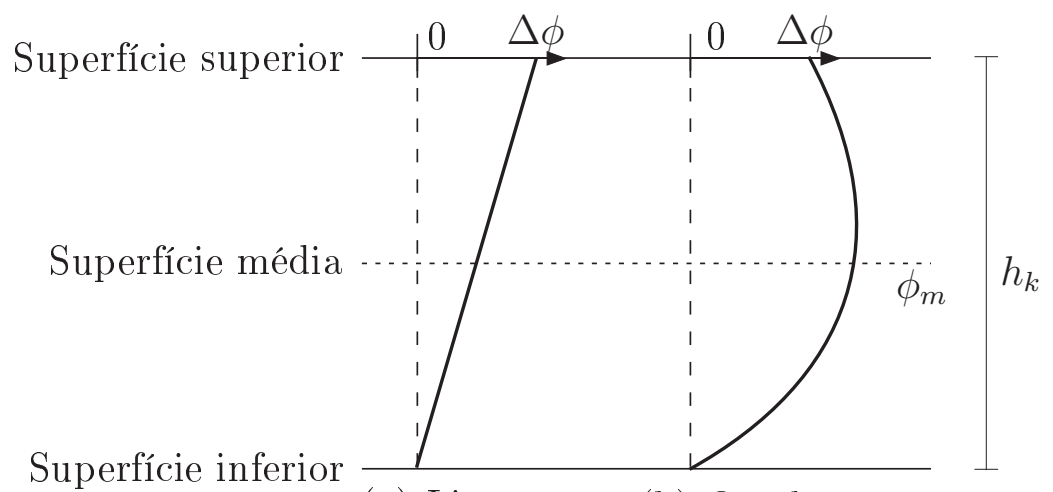

(a) Linear (b) Quadrática

Figura 2.6: Variação do potencial elétrico ao longo da espessura: (a) linear e (b) quadrática.

Assim, utilizando uma variação quadrática ao longo da espessura, tem-se que o potencial elétrico na camada piezelétrica pode ser escrito em termos das coordenadas naturais $(r, s, t)$ como sendo uma função da diferença de potencial elétrico entre os eletrodos da camada piezelétrica $\Delta \phi$ e do potencial elétrico da superfície média $\phi_{m}$ :

$$
\phi(r, s, t)=\frac{1}{2}\left(t+t^{2}\right) \Delta \phi+\left(1-t^{2}\right) \phi_{m}(r, s)
$$

Da Eq. (2.27) pode-se obter os potenciais elétricos das superfícies superior $(t=1)$, 
inferior $(t=-1)$ e da superfície média $(t=0)$ :

$$
\phi(t=1)=\Delta \phi \quad, \quad \phi(t=-1)=0 \quad, \quad \phi(t=0)=\phi_{m}
$$

assumindo-se que o eletrodo da superfície inferior é sempre aterrado. Discretizando-se a estrutura em elementos finitos, o potencial da superfície média $\phi_{m}$ pode ser interpolado da seguinte maneira:

$$
\phi_{m}=\sum_{k=1}^{8} N_{k}(r, s) \bar{\phi}_{m k}
$$

onde $N_{k}$ são as funções de forma (quadráticas) para um elemento de 8 nós e $\bar{\phi}_{m k}$ são os graus de liberdade de potencial elétrico nodal da superfície média da camada piezelétrica. Assim, o potencial elétrico dentro do elemento de casca piezelétrico é aproximado por:

$$
\phi(r, s, t)=\frac{1}{2}\left(t+t^{2}\right) \Delta \phi+\left(1-t^{2}\right) \sum_{k=1}^{8} N_{k}(r, s) \bar{\phi}_{m k}
$$

\subsection{Campo Elétrico}

Neste trabalho, faz-se a modelagem de estruturas piezelétricas finas de casca em qualquer lugar do espaço Tridimensional (3D). Assim, o campo elétrico (E) deve ser considerado nas três dimensões no sistema de coordenada do material $(1,2,3)$ (que no caso de materiais piezelétricos, esse sistema se iguala ao sistema de coordenadas locais $\left.\left(x^{\prime}, y^{\prime}, z^{\prime}\right)\right)$. O campo elétrico em cada direção é dado pelo gradiente do potencial elétrico (dado pela Eq. (2.30)). Para facilitar o entendimento, o campo elétrico é definido no sistema de coordenadas locais $\left(x^{\prime}, y^{\prime}, z^{\prime}\right)$. Como a posição de qualquer ponto da estrutura foi definida anteriormente (Eq. (2.9)) para o sistema de coordenadas globais $(x, y, z)$, faz-se necessária uma transformação de coordenadas. Assim, o campo elétrico pode ser escrito da seguinte maneira:

$$
\mathbf{E}(\phi)=\left\{\begin{array}{l}
E_{x^{\prime}} \\
E_{y^{\prime}} \\
E_{z^{\prime}}
\end{array}\right\}=-\left\{\begin{array}{l}
\frac{\partial \phi}{\partial x^{\prime}} \\
\frac{\partial \phi}{\partial y^{\prime}} \\
\frac{\partial \phi}{\partial z^{\prime}}
\end{array}\right\}=-\mathbf{T}_{\phi}\left\{\begin{array}{l}
\frac{\partial \phi}{\partial x} \\
\frac{\partial \phi}{\partial y} \\
\frac{\partial \phi}{\partial z}
\end{array}\right\}
$$

sendo que $\mathbf{T}_{\phi}$ é a matriz de transformação de coordenadas de potencial elétrico dada por

$$
\mathbf{T}_{\phi}=\left[\begin{array}{ccc}
l_{1} & m_{1} & n_{1} \\
l_{2} & m_{2} & n_{2} \\
l_{3} & m_{3} & n_{3}
\end{array}\right]
$$


onde $l_{i}, m_{i}$ e $n_{i}(i=1,2,3)$ são dados pela Eq. (2.23), e

$$
\left\{\begin{array}{l}
\frac{\partial \phi}{\partial x} \\
\frac{\partial \phi}{\partial y} \\
\frac{\partial \phi}{\partial z}
\end{array}\right\}=\mathbf{J}^{-1}\left\{\begin{array}{l}
\frac{\partial \phi}{\partial r} \\
\frac{\partial \phi}{\partial s} \\
\frac{\partial \phi}{\partial t}
\end{array}\right\}
$$

Derivando a Eq. (2.30) em relação à $r, s$ e $t$, e substituindo a Eq. (2.33) na Eq. (2.31), pode-se reescrever o campo elétrico como:

$$
\mathbf{E}(\phi)=-\mathbf{T}_{\phi} \mathbf{B}_{\phi} \phi_{e}
$$

sendo que $\mathbf{B}_{\phi}$ é a matriz de interpolações piezelétricas e $\phi_{e}$ é o vetor de potenciais elétricos no elemento, dados por:

$$
\mathbf{B}_{\phi}=\mathbf{J}^{-1}\left[\begin{array}{cc}
\left(1-t^{2}\right) \frac{\partial \mathbf{N}}{\partial r} & 0 \\
\left(1-t^{2}\right) \frac{\partial \mathbf{N}}{\partial s} & 0 \\
-2 t \mathbf{N} & (0.5+t)
\end{array}\right] \quad \text { e } \boldsymbol{\phi}_{e}=\left\{\begin{array}{c}
\phi_{m} \\
\Delta \phi
\end{array}\right\}_{e}
$$

onde $\mathbf{N}$ é o vetor de funções de forma e $\phi_{m}$ é o vetor de potenciais elétricos nodais da superfície média:

$$
\mathbf{N}=\left[\begin{array}{lll}
N_{1} & \ldots & N_{8}
\end{array}\right] \quad ; \quad \phi_{m}=\left[\begin{array}{lll}
\bar{\phi}_{m 1} & \ldots & \bar{\phi}_{m 8}
\end{array}\right]^{T}
$$

\subsection{Tensões Mecânicas}

Como já foi dito anteriormente, o sistema de coordenadas utilizado para calcular as tensões mecânicas é o sistema de coordenadas do material. Para materiais ortotrópicos, permite melhor identificação das tensões nas direções longitudinais e transversais à fibra. Para materiais piezelétricos e isotrópicos as tensões nos sistemas de coordenadas local e do material são iguais, já que os dois sistemas são coincidentes. Assim, substituindo a Eq. (2.26) de deformação e a Eq. (2.34) de campo elétrico na Eq. (2.1), a forma genérica para cálculo das tensões mecânicas, independente do tipo de material, pode ser escrito da seguinte maneira:

$$
\boldsymbol{\sigma}=\left\{\begin{array}{c}
\sigma_{11} \\
\sigma_{22} \\
\tau_{12} \\
\tau_{23} \\
\tau_{13}
\end{array}\right\}=\mathbf{c}^{E} \mathbf{T}_{\theta} \mathbf{T}_{\varepsilon} \mathbf{B}_{u} \mathbf{u}_{e}+\mathbf{e} \mathbf{T}_{\phi} \mathbf{B}_{\phi} \phi_{e}
$$


ou seja, para materiais piezelétricos e isotrópicos, utiliza-se $\theta=0^{\circ}$, e para materiais ortotrópicos e isotrópicos, utiliza-se a matriz e com valores nulos. Deve-se lembrar que está sendo considerado o estado plano de tensões, ou seja, $\sigma_{33}=0$.

Entretanto, a Eq. (2.37) é utilizada quando se tem um campo elétrico aplicado na estrutura, que é o caso de atuadores. No caso de sensores e coletores de energia onde o campo elétrico é nulo, a Eq. (2.37) fica:

$$
\boldsymbol{\sigma}=\mathbf{c}^{E} \mathbf{T}_{\theta} \mathbf{T}_{\varepsilon} \mathbf{B}_{u} \mathbf{u}_{e}
$$

Como este trabalho propõe utilizar o cálculo de tensões somente para sensores e coletores de energia, por simplificação, será utilizada apenas a Eq. (2.38) para representar as tensões no elemento deste ponto em diante. Deve-se ressaltar que $\boldsymbol{\sigma}$ não está escrito com índice pois ele representa a tensão em qualquer ponto do domínio. Utilizando o MEF, $\mathbf{u}_{e}$ representa os deslocamentos do elemento e que contém o ponto no qual a tensão está sendo calculada.

\subsection{Equações de Equilíbrio}

As equações governantes que descrevem o equilíbrio de um meio contínuo piezelétrico podem ser obtidas utilizando-se vários princípios variacionais. Neste trabalho, é utilizado o princípio de Hamilton que parte do princípio variacional para descrever o trabalho virtual exercido por forças externas mecânicas e elétricas (TIERSTEN, 1967; ALLIK; HUGHES, 1970; BALAMURUGAN; NARAYANAN, 2008). Assim, a forma integral da equação de equilíbrio estática, resultante do princípio variacional, pode ser escrita da seguinte maneira:

$$
\int_{\Omega}\left(\boldsymbol{\sigma}^{T} \delta \boldsymbol{\varepsilon}-\mathbf{D}^{T} \delta \mathbf{E}+\rho \ddot{\mathbf{u}}^{T} \delta \mathbf{u}\right) d \Omega-\int_{\Gamma_{1}} \mathbf{t}_{S}^{T} \delta \mathbf{u} d \Gamma-\mathbf{t}_{P}^{T} \delta \mathbf{u}+\mathbf{q}_{P}^{T} \delta \boldsymbol{\phi}=0
$$

onde $\mathbf{u}$ é o vetor de deslocamentos mecânicos, $\phi$ é o vetor de potenciais elétricos (mais de um domínio piezelétrico pode ser considerado) e $\delta$ é uma quantidade virtual. t e q são os vetores de forças mecânicas e cargas elétricas, respectivamente, e os subscritos $S$ e $P$ denotam carregamento distribuído (em uma superfície) ou concentrado (em pontos distintos), respectivamente. Assim, os termos $\int_{\Gamma_{1}} \mathbf{t}_{S}^{T} \delta \mathbf{u} d \Gamma, \mathbf{t}_{P}^{T} \delta \mathbf{u}$ e $\mathbf{q}_{P}^{T} \delta \boldsymbol{\phi}$ representam os trabalhos virtuais exercidos pela pressão na superfície $\Gamma_{1}$, pela força pontual e pela carga elétrica pontual, respectivamente. É importante ressaltar que, apesar da carga elétrica (ou potencial elétrico) ser aplicada numa superfície, ela é tratada nesse trabalho como carregamentos pontuais (em todos os elementos) e são acoplados de modo que toda 
superfície tenha o mesmo carregamento.

Assumindo regime harmônico $\left(\mathbf{u}=\mathbf{u}_{e} e^{i \omega t}, \phi=\phi_{e} e^{i \omega t}\right)$, com $\omega=2 \pi f$ e $f$ sendo a frequência de excitação, chega-se ao seguinte sistema de equações de equilíbrio piezelétrico, com amortecimento estrutural, na sua forma global:

$$
\left[\begin{array}{cc}
\mathbf{K}_{\mathbf{u u}}-\omega^{2} \mathbf{M}+i \omega \mathbf{C} & \mathbf{K}_{\mathbf{u} \phi} \\
\mathbf{K}_{\mathbf{u} \phi}^{T} & \mathbf{K}_{\phi \phi}+i \omega \mathbf{K}_{R}
\end{array}\right]\left\{\begin{array}{l}
\mathbf{U} \\
\boldsymbol{\Phi}
\end{array}\right\}=\left\{\begin{array}{l}
\mathbf{F} \\
\boldsymbol{\Psi}
\end{array}\right\} \text { ou } \mathbf{K} \overline{\mathbf{U}}=\overline{\mathbf{F}}
$$

onde $\overline{\mathbf{U}}$ é o vetor global de deslocamentos e potenciais elétricos e $\overline{\mathbf{F}}$ é o vetor global de forças mecânicas e cargas elétricas. A matrizes globais de rigidez $\left(\mathbf{K}_{\mathbf{u u}}, \mathbf{K}_{\mathbf{u} \phi}\right.$ e $\left.\mathbf{K}_{\phi \phi}\right)$, massa (M) e amortecimento (C) devem ser montadas a partir das suas respectivas matrizes locais de cada elemento, as quais são definidas na Tabela 2.1, sendo que $\alpha_{D}$ e $\beta_{D}$ são os coeficientes de amortecimento de Rayleigh e $\rho$ é a densidade do material utilizado.

A matriz $\mathbf{K}_{R}$ é a matriz do resistor elétrico e é escrita de seguinte maneira (RUPP et al., 2009):

$$
\mathbf{K}_{R}=\left[\begin{array}{cc}
\frac{1}{\omega^{2} R} & \mathbf{0} \\
\mathbf{0} & \mathbf{0}
\end{array}\right]
$$

onde o valor $1 / \omega^{2} R$ está posicionado no grau de liberdade elétrico no qual o resistor está conectado e $R$ é o valor da resistência elétrica utilizada.

\begin{tabular}{|c|c|c|}
\hline $\mathbf{K}_{\mathbf{u u}}^{e}$ & $=\int_{\Omega^{e}} \mathbf{B}_{\mathbf{u}}^{T} \mathbf{T}_{\varepsilon}^{T} \mathbf{T}_{\theta}^{T} \mathbf{c}^{E} \mathbf{T}_{\theta} \mathbf{T}_{\varepsilon} \mathbf{B}_{\mathbf{u}} d \Omega$ & Matriz de rigidez mecânica \\
\hline $\mathbf{K}_{\mathbf{u} \phi}^{e}$ & $=\int_{\Omega^{e}} \mathbf{B}_{\mathbf{u}}^{T} \mathbf{T}_{\varepsilon}^{T} \mathbf{T}_{\theta}^{T} \mathbf{e} \mathbf{T}_{\phi} \mathbf{B}_{\phi} d \Omega$ & Matriz de rigidez piezelétrica \\
\hline $\mathbf{K}_{\phi \mathbf{u}}^{e}$ & $=\mathbf{K}_{\mathbf{u} \phi}^{e^{T}}$ & \\
\hline $\mathbf{K}_{\phi \phi}^{e}$ & $=-\int_{\Omega^{e}} \mathbf{B}_{\phi}^{T} \mathbf{T}_{\phi}^{T} \boldsymbol{\epsilon} \mathbf{T}_{\phi} \mathbf{B}_{\phi} d \Omega$ & Matriz de rigidez dielétrica \\
\hline $\mathbf{M}^{e}$ & $=\rho \int_{\Omega^{e}} \mathbf{A}^{T} \mathbf{A} d \Omega$ & Matriz de massa consistente \\
\hline $\mathrm{C}^{e}$ & $=\alpha_{D} \mathbf{M}^{e}+\beta_{D} \mathbf{K}_{\mathbf{u u}}^{e}$ & Matriz de amortecimento \\
\hline
\end{tabular}

Tabela 2.1: Definição das matrizes e vetores eletroelásticos.

No projeto de atuadores piezocompósitos, a excitação elétrica é feita por potencial elétrico, ou seja, é aplicada tensão elétrica $(V)$ nos eletrodos das camadas piezelétricas, que corresponde ao grau de liberdade elétrico $\Delta \phi$ (ver seção 2.3). Assim, para resolver o sistema de equações da Eq. (2.40), é necessário prescrever o potencial elétrico cuja 
descrição é apresentada na seção 4.2 .

Já no projeto de coletores de energia, utiliza-se excitação harmônica de movimento, ou seja, a estrutura vibra devido a deslocamentos prescritos em regiões pré-estabelecidas da estrutura. Assim, deve-se prescrever os deslocamentos conhecidos utilizando a formulação descrita na seção 6.1. Além disso, neste trabalho, são considerados coletores de energia com um resistor acoplado à estrutura piezelétrica. Esse resistor tem a finalidade de representar um dispositivo eletrônico cuja alimentação elétrica é proveniente dos coletores de energia.

\subsection{Teoria "Layer-Wise" para Acoplamento entre Camadas}

Neste trabalho, as estruturas a serem modeladas são estruturas de casca piezocompósitas, ou seja, camadas de diferentes materiais são acopladas entre si. Dentre as diversas abordagens para se modelar estruturas de casca laminadas, duas se destacam: a Teoria Laminar Clássica (ou "Classical Laminate Theory" - CLT) (REDDY, 2006) e a Teoria de "Zig-zag" (ou teoria "Layer-wise") (KÖGL; BUCALEM, 2005a). Uma configuração de estrutura de casca piezocompósita é apresentada na Fig. 2.7.

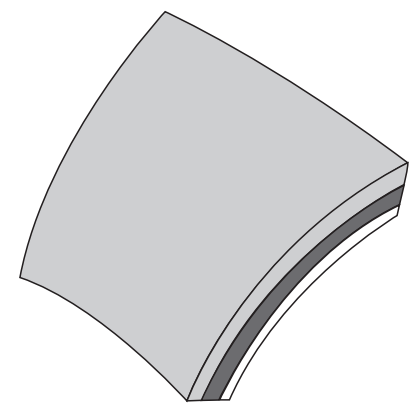

Figura 2.7: Representação de uma estrutura piezocompósita de casca.

Na Teoria Laminar Clássica, o composto é considerado como se fosse apenas uma camada, gerando uma variação suave no campo de deslocamentos ao longo da espessura do compósito (ver Fig. 2.8(a)). Nessa abordagem, os graus de liberdade são definidos na superfície média do composto e não em cada camada.

Na Teoria de "Zig-zag", cada tipo diferente de material é considerado como uma camada distinta do composto. Nessa abordagem, os graus de liberdade são definidos nas superfícies médias de cada camada, gerando um campo de deslocamentos com variação abrupta nas interfaces (ver Fig. 2.8(b)). Essa abordagem, apesar de aumentar o custo 
computacional, apresenta resultados mais precisos e dá a possibilidade de se aplicar diferentes potenciais elétricos em cada camada piezelétrica.

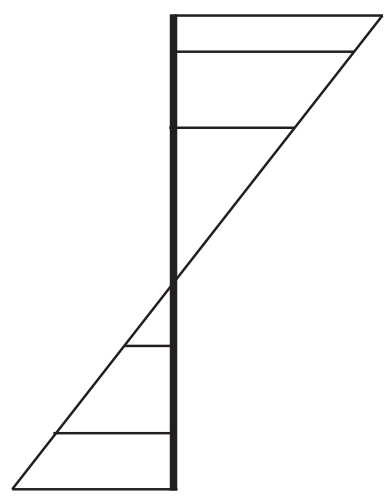

(a) Teoria Laminar Clássica

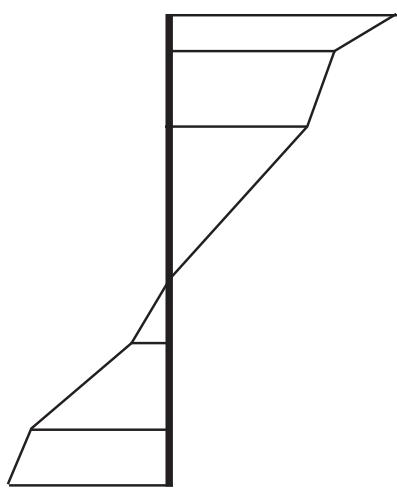

(b) Teoria de "Zig-zag"

Figura 2.8: Deformações ao longo da espessura de cascas compostas.

Assim, este projeto utiliza a teoria de "Zig-zag" proposta por Kögl e Bucalem (2005a) por se tratar de estruturas piezocompósitas e haver a liberdade de se aplicar diferentes potenciais elétricos em cada camada piezelétrica.

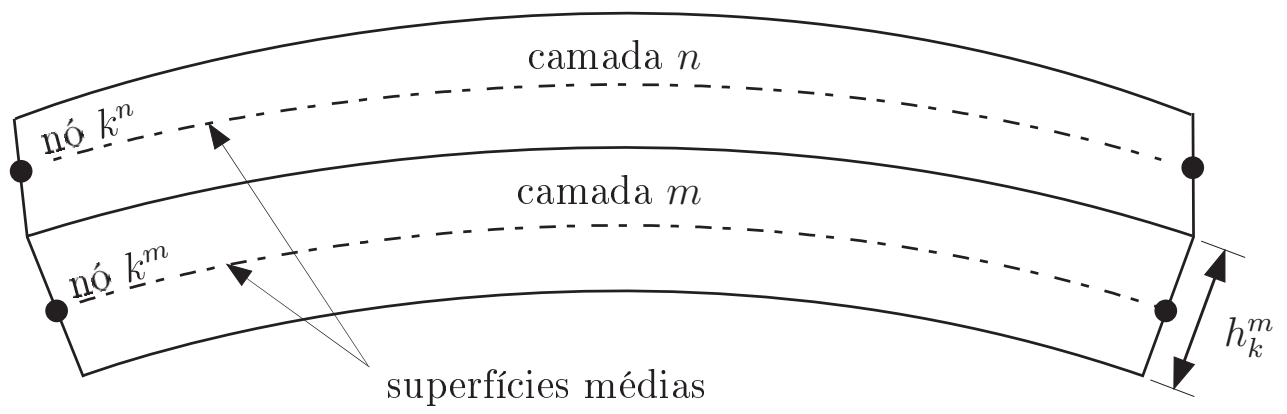

Figura 2.9: Adesão entre duas camadas $m$ e $n$.

Considerando, então, que duas camadas $m$ e $n$ estão perfeitamente aderidas uma à outra, como mostrado na Fig. 2.9, o campo de deslocamentos na interface, apesar de ter uma variação abrupta, deve ser contínua. Baseando-se nas coordenadas naturais $(r, s, t)$, os deslocamentos de ambas as camadas, na interface, podem ser escritos como:

$$
\left\{\begin{array}{l}
u^{m} \\
v^{m} \\
w^{m}
\end{array}\right\}_{(t=1)}=\left\{\begin{array}{c}
u^{n} \\
v^{n} \\
w^{n}
\end{array}\right\}_{(t=-1)}
$$

Como os deslocamentos são calculados diretamente nos nós, as funções de forma da Eq. (2.10) são iguais a 1 somente para o nó em questão e zeros para os outros nós. Assim, 
os deslocamentos das camadas $m$ e $n$ podem ser escritos como:

$$
\begin{aligned}
& \left\{\begin{array}{l}
u^{m} \\
v^{m} \\
w^{m}
\end{array}\right\}_{(t=1)}=\left[\begin{array}{ccccc}
1 & 0 & 0 & -\frac{h_{k}^{m}}{2} V_{2 x}^{k, m} & \frac{h_{k}^{m}}{2} V_{1 x}^{k, m} \\
0 & 1 & 0 & -\frac{h_{k}^{m}}{2} V_{2 y}^{k, m} & \frac{h_{k}^{m}}{2} V_{1 y}^{k, m} \\
0 & 0 & 1 & -\frac{h_{k}^{m}}{2} V_{2 z}^{k, m} & \frac{h_{k}^{m}}{2} V_{1 z}^{k, m}
\end{array}\right]\left\{\begin{array}{c}
u_{k}^{m} \\
v_{k}^{m} \\
w_{k}^{m} \\
\alpha_{k}^{m} \\
\beta_{k}^{m}
\end{array}\right\}=\overline{\mathbf{A}}^{k, m} \hat{\mathbf{u}}_{k}^{m} \\
& \left\{\begin{array}{c}
u^{n} \\
v^{n} \\
w^{n}
\end{array}\right\}_{(t=-1)}=\left[\begin{array}{lllll}
1 & 0 & 0 & \frac{h_{k}^{n}}{2} V_{2 x}^{k, n} & -\frac{h_{k}^{n}}{2} V_{1 x}^{k, n} \\
0 & 1 & 0 & \frac{h_{k}^{n}}{2} V_{2 y}^{k, n} & -\frac{h_{k}^{n}}{2} V_{1 y}^{k, n} \\
0 & 0 & 1 & \frac{h_{k}^{n}}{2} V_{2 z}^{k, n} & -\frac{h_{k}^{n}}{2} V_{1 z}^{k, n}
\end{array}\right]\left\{\begin{array}{c}
u_{k}^{n} \\
v_{k}^{n} \\
w_{k}^{n} \\
\alpha_{k}^{n} \\
\beta_{k}^{n}
\end{array}\right\}=\overline{\mathbf{A}}^{k, n} \hat{\mathbf{u}}_{k}^{n}
\end{aligned}
$$

Substituindo as Eqs. (2.43) e (2.44) na Eq. (2.42), tem-se a seguinte equação de acoplamento:

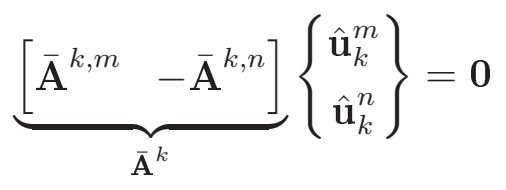

que pode ser introduzida na equação de equilíbrio (Eq. (2.40)) através dos multiplicadores de Lagrange da seguinte maneira (BATHE, 1996):

$$
\left[\begin{array}{ccc}
\mathbf{K}_{\mathbf{u u}}-\omega^{2} \mathbf{M}+i \omega \mathbf{C} & \mathbf{K}_{\mathbf{u} \phi} & \overline{\mathbf{A}}^{T} \\
\mathbf{K}_{\mathbf{u} \phi}^{T} & \mathbf{K}_{\phi \phi}+i \omega \mathbf{K}_{R} & \mathbf{0} \\
\overline{\mathbf{A}} & \mathbf{0} & \mathbf{0}
\end{array}\right]\left\{\begin{array}{l}
\mathbf{U} \\
\mathbf{\Phi} \\
\boldsymbol{\lambda}
\end{array}\right\}=\left\{\begin{array}{l}
\mathbf{F} \\
\boldsymbol{\Psi} \\
\mathbf{0}
\end{array}\right\}
$$




\section{MÉTODO DE OTIMIZAÇÃO TOPOLÓGICA}

Neste capítulo é apresentado o método de otimização topológica utilizado juntamente com o conceito do modelo de material adotado para otimizar simultaneamente a topologia, a polarização (do material piezelétrico) e a orientação das fibras (do material ortotrópico), considerando restrição de falha mecânica. É também apresentada a implementação do método de otimização utilizado nesta tese por meio de um fluxograma. Os conceitos básicos do MOT, como tipos de otimização estrutural, Domínio Fixo Estendido (DFE) e instabilidades numéricas, são descritos no Apêndice D.

\subsection{Introdução ao MOT}

A OT é uma técnica eficiente para a otimização estrutural que consiste em distribuir material dentro de uma região conhecida (a DFE), com o objetivo de satisfazer os requisitos do projeto e melhorar o desempenho da estrutura. Basicamente a OT combina técnicas de otimização com métodos de análise estrutural (MEF) para, iterativamente, obter o resultado de topologia ótima. A primeira implementação de otimização topológica surgiu com Bendsøe e Kikuchi (1988) que propuseram o método da homogeneização para maximizar a rigidez de estruturas com restrição de volume de material. O método da homogeneização se baseia em projetar células unitárias de uma microestrutura a partir de parâmetros $(\alpha, \beta$ e $\theta)$ que definem um espaço vazio dentro dessas células (ver Fig. 3.1), para então calcular suas propriedades mecânicas efetivas. Assim, os autores sugerem que o complicado problema de otimização topológica pode ser simplificado por um problema discreto de otimização paramétrica onde as variáveis de projeto são os parâmetros $\alpha, \beta$ e $\theta$. Entretanto, ao criar essas microestruturas porosas, o resultado da OT acaba por tornar o processo de fabricação inviável.

Em seguida, Bendsøe (1989) propôs um método baseado na distribuição de 


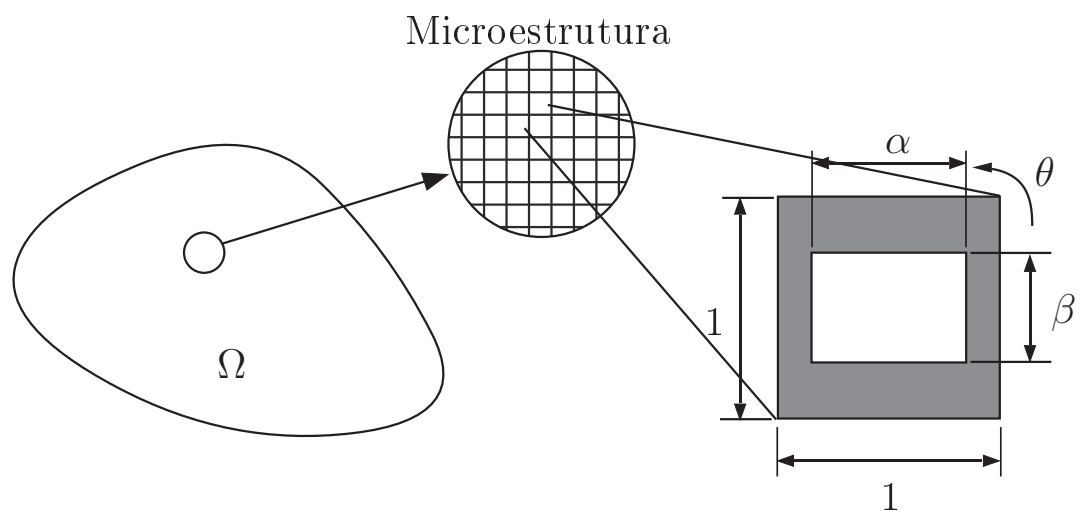

Figura 3.1: Modelo de microestrutura para o método da homogeneização.

pseudodensidades na microstrutura, chamada de método das densidades. As variáveis de projeto são as pseudo-densidades de cada elemento da malha de elementos finitos, que podem variar de 0 (vazio) a 1 (sólido), conforme um modelo de material adotado. $\mathrm{O}$ modelo de material mais conhecido e largamente utilizado é o SIMP (BENDSØE, 1989; ROZVANY; ZHOU; BIRKER, 1992) pela sua simplicidade de implementação e pela boa compatibilidade com o MEF. Assim como o método da homogeneização, o método das densidades também apresenta instabilidades numéricas, tais como instabilidade de tabuleiro, dependência de malha e controle de membro mínimo, as quais podem ser evitadas utilizando técnicas como filtros espaciais (SIGMUND; PETERSSON, 1998), controle de perímetro (JOG; HABER, 1996), projeção (BRUNS; TORTORELLI, 2001; GUEST; PREVOST; BELYTSCHKo, 2004), entre outros. Assim, o SIMP tem se mostrado bastante robusto em diversas aplicações como por exemplo, problemas elásticos lineares (BENDSøE; SIGMUND, 2003) e não-lineares estáticos (BRUNS; TORTORELLI, 2001; STEGMANN; LUND, 2005b), problemas dinâmicos (DIAZ; KIKUCHI, 1992; MA; KIKUCHI; CHENG, 1995) e sistemas multifísicos (KÖGL; SILVA, 2005; CARBONARI; SILVA; NISHIWAKI, 2005).

Esses dois métodos descritos anteriormente se baseiam na abordagem de microescala, ou seja, projetam a microestrutura do material. Existem outros métodos que são baseados na abordagem de macro escala, que utiliza uma distribuição discreta durante todo o processo de otimização e é baseado na geometria de funções de distribuição de material. Os principais métodos baseados na geometria são: o método evolucionário ("Evolutionary Structural Optimization" (ESO)) (XIE; STEVEN, 1993, 1994), onde a topologia ótima da estrutura pode ser representada pela adição ou remoção de elementos da malha de MEF utilizando critérios heurísticos; o método de derivada topológica (EECHENAUER; KOBELEV; SCHUMACHER, 1994; NOVOTNY et al., 2003), que modifica a topologia da estrutura alocando 'buracos' no domínio; e os métodos "level-set" (SETHIAN; WIEGMANN, 2000; ALlAIRE; JOUVE; TOADER, 2004) e o "phase-field" (TAKEZAWA; 
NISHIWAKI; KITAMURA, 2010; GAIN; PAULINO, 2012), que definem os contornos da topologia da estrutura baseando-se em curvas de nível.

\subsection{Formulação Adotada}

Neste trabalho, são consideradas as otimizações de topologia, de polarização (do material piezelétrico) e de orientação do ângulo das fibras do material ortotrópico, sujeita a uma restrição de falha mecânica. Assim, é necessário que a formulação de OT adotada considere três tipos diferentes de variáveis de projeto e que permita calcular as tensões mecânicas adequadamente. Como nenhum trabalho anterior aborda esses três tipos de otimização simultaneamente, propõe-se um novo método que combina formulações que consideram cada tipo de otimização separadamente.

O primeiro trabalho envolvendo otimização simultânea de topologia e de polarização do material piezelétrico foi apresentado por Kögl e Silva (2005) que aplicaram o MOT para o projeto de transdutores piezelétricos de casca e placa. Baseados no método das densidades, mais especificamente no modelo SIMP, os autores propõem o modelo de material PEMAP-P, que utiliza a mesma abordagem do SIMP para otimizar a distribuição de material e adiciona uma nova variável de projeto à propriedade piezelétrica para modificar o sentido da polarização do material piezelétrico para estruturas de placa e casca.

Já Stegmann e Lund (2005a) propõem um modelo para otimização simultânea de topologia e de orientação de fibras para maximizar a rigidez de estruturas de cascas. Eles denominaram esse modelo de Otimização de Material Discreto (OMD), ou "Discrete Material Optimization" (DMO) como é conhecido na literatura, e também se baseiam no modelo SIMP para penalizar a contribuição dos ângulos no cálculo das propriedades efetivas do material.

Para problemas envolvendo restrição de falha mecânica, são encontrados diversos trabalhos tratando da relaxação do espaço de soluções para contornar algumas dificuldades inerentes ao problema do cálculo de tensões na OT (CHENG; GUO, 1997; ROZVANY; SOBIESZCZANSKI-SOBIESKI, 1992; DUYSINX; BENDSØE, 1998; BRUGGI, 2008; LE et al., 2010). Esses trabalhos também baseiam-se nos métodos das densidades, e alguns deles especificamente utilizam o modelo SIMP (DUYSINX; BENDSøE, 1998; BRUGGI, 2008; LE et al., 2010).

Por esse motivo é adotada uma formulação baseada no método das densidades, 
combinando os modelos PEMAP-P e OMD para realizar a otimização de topologia, polarização e orientação de fibras simultaneamente, e o modelo SIMP para calcular as tensões mecânicas no domínio de projeto. A combinação desses 3 modelos é uma das contribuições científicas deste trabalho com relação ao MOT.

Dentro do DFE, a distribuição de material deve ser parametrizada para que a OT possa determinar a existência ou a ausência de material em cada ponto. Neste trabalho, que utiliza o método de densidades, a distribuição de material é parametrizada pelas pseudodensidades, representadas por $\gamma$. Assim, as pseudodensidades são parâmetros com valores entre 0 e 1 , e definem se um ponto é sólido, vazio ou possui densidades intermediárias. As pseudodensidades são utilizadas para calcular as matrizes de rigidez e massa, e portanto, são responsáveis pela topologia da estrutura.

\subsubsection{PEMAP-P}

Para análises estáticas piezelétricas, resultados quase preto-e-branco (sem materiais intermediários) são obtidos utilizando o modelo de material PEMAP-P proposto por Kögl e Silva (2005). O modelo PEMAP-P baseia-se principalmente no modelo SIMP (BENDSøE, 1989; ROZVANY; ZHOU; BIRKER, 1992) para modelar a distribuição de material e adiciona uma outra variável de projeto, que neste trabalho é chamada de variável de polarização $\varrho$, utilizada para modelar o sentido de polarização dos materiais piezelétricos. Assim, é possível levar em consideração tanto a otimização de material piezelétrico, quanto o sentido de polarização de cada ponto, ao invés de deixá-lo fixo em todo o processo. Desse modo, cria-se um grau de liberdade a mais na obtenção dos resultados de transdutores piezelétricos. Assim, as matrizes de propriedades efetivas elástica $\mathbf{c}^{E}$, piezelétrica e e dielétrica $\boldsymbol{\epsilon}^{S}$ podem ser calculadas, em qualquer ponto $\mathbf{x}$ do domínio, pelas seguintes expressões:

$$
\begin{gathered}
\mathbf{c}^{E}(\mathbf{x})=\gamma^{p_{c}}(\mathbf{x}) \mathbf{c}_{0}^{E} \\
\mathbf{e}(\mathbf{x})=\gamma^{p_{e}}(\mathbf{x})(2 \varrho(\mathbf{x})-1)^{p_{i}} \mathbf{e}_{0} \\
\boldsymbol{\epsilon}^{S}(\mathbf{x})=\gamma^{p_{\epsilon}}(\mathbf{x}) \boldsymbol{\epsilon}_{0}^{S}
\end{gathered}
$$

onde $\mathbf{c}_{0}^{E}$, $\mathbf{e}_{0}$ e $\boldsymbol{\epsilon}_{0}^{S}$ são as matrizes de propriedades base do material. Para qualquer tipo de material não-piezelétrico, $\mathbf{e}_{0}$ e $\boldsymbol{\epsilon}_{0}^{S}$ são matrizes nulas. $p_{c}, p_{e}$ e $p_{\epsilon}$ são os coeficientes de penalização utilizados para recuperar o caráter discreto da solução, ou seja, com reduzida quantidade de material intermediário na distribuição de material. $p_{i}$ é o coeficiente de penalização para a polarização do material piezelétrico, que deve ser um número 
ímpar para permitir valores negativos de polarização. Nota-se que o modelo PEMAP-P é basicamente o modelo SIMP para distribuição de material, adotando coeficientes de penalização diferentes para cada propriedade, adicionando um modelo para alterar o sinal de polarização somente na propriedade piezelétrica.

Neste trabalho, o modelo PEMAP-P descrito acima é utilizado tanto para análises estáticas quanto dinâmicas. Entretanto, para as análises dinâmicas é necessário adotar um modelo de material para calcular a matriz de massa da estrutura. Assim, seguindo a formulação do PEMAP-P, a densidade do material, em qualquer ponto $\mathbf{x}$ do domínio, é calculada da seguinte maneira:

$$
\rho(\mathbf{x})=\gamma^{p_{m}}(\mathbf{x}) \rho_{0}
$$

onde $\rho_{0}$ é a densidade do material utilizado e $p_{m}$ é o coeficiente de penalização da massa.

A escolha dos valores dos coeficientes de penalização pode ser feita arbitrariamente, seguindo o critério do projetista ou, em casos específicos, seguir critérios próprios de forma a garantir a convergência de solução (KIM et al., 2010).

Ao discretizar o domínio em uma malha de elementos finitos, as pseudodensidades $(\gamma)$ e as variáveis de polarização ( $\varrho$ ) são atribuídas a cada elemento $e$, tendo valor constante dentro deste. Dessa forma, as propriedades dos materiais (Eqs. (3.1) a (3.4)) são também calculadas para cada elemento, podendo ser reescritas da seguinte forma:

$$
\begin{gathered}
\mathbf{c}_{e}^{E}=\gamma_{e}^{p_{c}} \mathbf{c}_{0}^{E} \\
\mathbf{e}_{e}=\gamma_{e}^{p_{e}}\left(2 \varrho_{e}-1\right)^{p_{i}} \mathbf{e}_{0} \\
\boldsymbol{\epsilon}_{e}^{S}=\gamma_{e}^{p_{\epsilon}} \boldsymbol{\epsilon}_{0}^{S} \\
\rho_{e}=\gamma_{e}^{p_{m}} \rho_{0}
\end{gathered}
$$

Assim, substituindo as Eqs. (3.5) a (3.8) nas matrizes de rigidez e massa da Tab. 2.1 tem-se que:

$$
\begin{aligned}
& \mathbf{K}_{\mathbf{u u}}^{e}=\gamma_{e}^{p_{c}} \int_{\Omega^{e}} \mathbf{B}_{\mathbf{u}}^{T} \mathbf{T}_{\varepsilon}^{T} \mathbf{T}_{\theta}^{T} \mathbf{c}_{0}^{E} \mathbf{T}_{\theta} \mathbf{T}_{\varepsilon} \mathbf{B}_{\mathbf{u}} d \Omega \\
& \mathbf{K}_{\mathbf{u} \phi}^{e}=\gamma_{e}^{p_{e}}\left(2 \varrho_{e}-1\right)^{p_{i}} \int_{\Omega^{e}} \mathbf{B}_{\mathbf{u}}^{T} \mathbf{T}_{\varepsilon}^{T} \mathbf{T}_{\theta}^{T} \mathbf{e}_{0} \mathbf{T}_{\phi} \mathbf{B}_{\phi} d \Omega \\
& \mathbf{K}_{\phi \phi}^{e}=-\gamma_{e}^{p_{\epsilon}} \int_{\Omega^{e}} \mathbf{B}_{\phi}^{T} \mathbf{T}_{\phi}^{T} \boldsymbol{\epsilon} \mathbf{T}_{\phi} \mathbf{B}_{\phi} d \Omega \\
& \mathbf{M}^{e}=\gamma_{e}^{p_{m}} \rho_{0} \int_{\Omega^{e}} \mathbf{A}^{T} \mathbf{A} d \Omega
\end{aligned}
$$


sendo que a pseudo-densidade $\gamma_{e}$ e o termo $\left(2 \varrho_{e}^{p_{i}}-1\right)$ podem ser retirados da integral por serem variáveis escalares, representando um valor constante dentro de todo o elemento $e$.

A escolha dos coeficientes de penalização do modelo de material $p_{c}, p_{e}, p_{\epsilon}$ e $p_{i}$ é de extrema importância para se obter um resultado de qualidade em termos de quantidade de materiais intermediários. Kögl e Silva (2005) e Silva e Kögl (2004) mostram que resultados quase sem materiais intermediários são obtidos utilizando $p_{\epsilon}=p_{i}=1$ e $p_{c}=p_{e}=3$, cujos valores foram obtidos empiricamente. Kim et al. (2010) apresenta um estudo sobre os valores desses coeficientes no modelo PEMAP-P e conclui que existe uma relação entre os valores desses coeficientes. Entretanto, nos testes realizados neste trabalho, as relações entre os valores dos coeficientes apresentados por Kim et al. (2010) não apresentaram nenhuma melhora nos resultados, prejudicando a convergência dos resultados em alguns casos. Assim, os valores dos coeficientes de penalização do modelo PEMAP-P utilizados neste trabalho são baseados no trabalho de Kögl e Silva (2005) e Silva e Kögl (2004).

\subsubsection{Otimização de Orientação de Fibras utilizando o Método OMD}

Como já foi dito anteriormente, além de materiais isotrópicos e piezelétricos, são utilizados também materiais ortotrópicos, cujas propriedades elásticas dependem da orientação do ângulo da fibra. O ângulo da fibra é representado por $\theta$ e a matriz de rotação ortotrópica é dada pela Eq. (2.25). A matriz de rigidez elástica $\mathbf{K}_{\mathbf{u u}}^{e}$ apresentada na Eq. (3.9) já considera a rotação ortotrópica nas propriedades elásticas pelo termo $\mathbf{T}_{\theta}^{T} \mathbf{c}_{0}^{E} \mathbf{T}_{\theta}$. Assim, pode-se definir $\mathbf{c}_{\theta}^{E}$ como a matriz de propriedades elásticas rotacionada da seguinte maneira:

$$
\mathbf{c}_{\theta}^{E}=\mathbf{T}_{\theta}^{T} \mathbf{c}_{0}^{E} \mathbf{T}_{\theta}
$$

lembrando que a matriz de rotação ortotrópica $\mathbf{T}_{\theta}$ é obtida utilizando-se a Eq. (2.25)

A ideia principal é otimizar os ângulos das fibras em cada elemento de modo a atingir um melhor desempenho da estrutura, ou seja, a cada novo valor de $\theta$, uma nova matriz de propriedades $\mathbf{c}_{\theta}^{E}$ deve ser calculada e a rigidez da estrutura é modificada. Entretanto, se considerar o ângulo das fibras como um parâmetro contínuo no processo de otimização, ou seja, $\theta$ variando entre $-90^{\circ}$ e $+90^{\circ}$, por exemplo, o espaço de soluções se torna não-convexo, e portanto, seria necessário lidar com um problema com múltiplos mínimos locais (STEGMANN; LUND, 2005a; LUND, 2009). Neste trabalho, é utilizada a formulação proposta por Lund e co-autores (STEGMANN; LUND, 2005a; LUND, 2009) chamada de 
OMD, ou DMO como conhecido na literatura, onde utilizam a estratégia de combinar diferentes tipos de materiais, sugerido por Sigmund e coautores (SIGMUND; TORQUATO, 1997), onde a propriedade efetiva do material final é calculada como uma somatória ponderada dos diferentes materiais candidatos. A partir dessa ideia, Lund e coautores sugerem que a matriz de propriedades elásticas efetiva $\mathbf{c}_{e f}^{E}$ deve ser calculada como uma soma ponderada de um número finito $n^{c}$ de matrizes constitutivas $\mathbf{c}_{\theta_{i}}^{E}$, com $i=1, \ldots, n^{c}$, da seguinte maneira (STEGMANN; LUND, 2005a):

$$
\mathbf{c}_{e f}^{E}=\sum_{i=1}^{n^{c}} w_{o_{i}} \mathbf{c}_{\theta_{i}}^{E}=w_{o_{1}} \mathbf{c}_{\theta_{1}}^{E}+w_{o_{2}} \mathbf{c}_{\theta_{2}}^{E}+\cdots+w_{o_{n} c} \mathbf{c}_{\theta_{n^{c}}}^{E}, \quad 0 \leq w_{o_{i}} \leq 1
$$

sendo que cada $\mathbf{c}_{\theta_{i}}^{E}$ representa o mesmo tipo de material com as fibras orientadas em diferentes ângulos discretos $\theta_{i}$ e pode ser obtida utilizando-se a Eq. (3.13). $w_{o_{i}}$ são os coeficientes de peso, cujos valores devem estar entre 0 e 1, e que vão determinar qual ângulo candidato $i$ deve ser escolhido para cada elemento.

Para utilizar essa formulação, a matriz de rigidez elástica $\mathbf{K}_{\mathbf{u u}}^{e}$, apresentada na Eq. (3.9), deve ser reescrita da seguinte forma:

$$
\mathbf{K}_{\mathbf{u u}}^{e}=\gamma_{e}^{p_{c}} \int_{\Omega^{e}} \mathbf{B}_{\mathbf{u}}^{T} \mathbf{T}_{\varepsilon}^{T} \mathbf{c}_{e f}^{E} \mathbf{T}_{\varepsilon} \mathbf{B}_{\mathbf{u}} d \Omega
$$

ou seja, $\mathbf{c}_{e f}^{E}$ substitui o termo $\mathbf{T}_{\theta}^{T} \mathbf{c}_{0}^{E} \mathbf{T}_{\theta}$.

O objetivo da otimização é escolher somente uma dessas matrizes constitutivas em cada elemento, fazendo com que apenas um dos coeficientes $w_{o_{i}}$ tenha valor igual a 1 e os demais iguais a zero. No início da otimização, $\mathbf{c}_{e f}^{E}$ é formado pela contribuição de todos os materiais candidatos, ou seja, é uma mistura de todos os ângulos. No final da otimização, a parametrização dos coeficientes de peso devem cumprir a exigência de que apenas um ângulo candidato seja escolhido (LUND, 2009).

Para alcançar esse objetivo, mais um tipo de variável de projeto deve ser definido. Essa variável é chamada, neste trabalho, de variável de orientação, representada por $\vartheta_{i}^{e}$, cujo valor varia de 0 a 1 . Os índices $i$ e $e$ indicam, respectivamente, o ângulo candidato e o elemento no qual ele é definido. Dessa forma, para cada elemento da camada de material ortotrópico, são associadas $n^{c}$ variáveis de orientação, o que gera um grande número de variáveis mas evita o problema de múltiplos mínimos locais (STEGMANN; LUND, 2005a; LUND, 2009). Assim, os coeficientes de peso $w_{o_{i}}$ são calculados da seguinte maneira:

$$
w_{o_{i}}=\frac{\hat{w}_{i}}{\sum_{k=1}^{n^{c}} \hat{w}_{k}}, \text { onde } \hat{w}_{i}=\left(\vartheta_{i}^{e}\right)^{p_{\vartheta}} \prod_{j=1, j \neq i}^{n^{c}}\left(1-\left(\vartheta_{j}^{e}\right)^{p_{\vartheta}}\right)
$$


Para tentar aproximar as variáveis $\vartheta_{i}^{e}$ para os valores 0 ou 1, é utilizado o coeficiente de penalização $p_{\vartheta}$ para penalizar os valores intermediários, assim como no modelo SIMP. $p_{\vartheta}$ é normalmente igual a 2 no início da otimização e, a cada 10 iterações, adiciona-se uma unidade até que $p_{\vartheta}=8$. Além disso, o termo $1-\left(\vartheta_{j}^{e}\right)^{p_{\vartheta}}$ é introduzido de modo que um acréscimo em $\vartheta_{i}^{e}$ resulta numa penalização para reduzir os coeficientes dos outros candidatos. O método OMD para otimização do ângulo das fibras está ilustrado na Fig. 3.2.

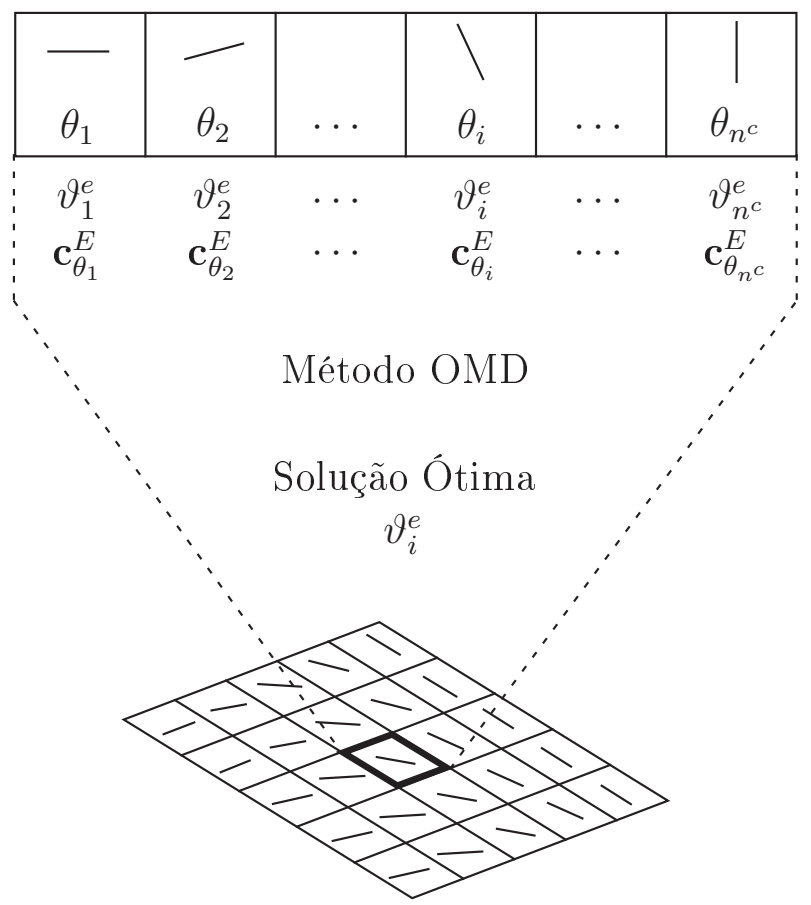

Figura 3.2: Método OMD para otimização de ângulo, onde $\theta_{i}$ é o ângulo candidato, $\vartheta_{i}$ e $\mathbf{c}_{\theta_{i}}^{E}$ são, respectivamente, a variável de orientação e a matriz constitutiva associada ao ângulo $i$, e $n^{c}$ é o número total de ângulos candidatos.

\subsubsection{Variáveis de Projeto Nodais e Técnica de Projeção}

Este trabalho utiliza a abordagem proposta por Guest, Prevost e Belytschko (2004) que adota variáveis de projeto nos nós da malha de elementos finitos e utiliza a técnica de projeção linear proposta por Bruns e Tortorelli (2001) para calcular as pseudodensidades nos elementos. Com esta abordagem, Guest, Prevost e Belytschko (2004) mostram resultados que não apresentam dependência de malha nem padrões de tabuleiro, porém eles também mostram que não é possível evitar o aparecimento de materiais intermediários ao utilizar a técnica de projeção linear, já que essa técnica faz uma média ponderada dos valores vizinhos ao elemento em questão. Então, para contornar este problema, os autores complementam a técnica de projeção com uma função "degrau", ou "Heaviside" 
como é denominado por Guest, Prevost e Belytschko (2004), fazendo com que as pseudodensidades intermediárias tendam a 1 e que somente valores bem próximos a zero representem elementos vazios.

Primeiramente, define-se $d$ nos nós da malha de elementos finitos como sendo as variáveis de projeto que representam parâmetros independentes e que são utilizadas para a determinação das pseudodensidades $\gamma_{e}$ em cada elemento, que por sua vez determinam a topologia da estrutura. Por isso, neste projeto, $d$ é denominada de variável topológica, já que existem outros dois tipos de variáveis de projeto (de polarização e de orientação). Entretanto, apesar deste trabalho utilizar o elemento de casca de 8 nós, as variáveis topológicas $d$ são atribuídas apenas nos vértices dos elementos com o intuito de reduzir o custo computacional sem perda de resolução nos resultados.

Em seguida, é necessário identificar os nós que influenciam o valor da pseudodensidade do elemento $e$. A função de projeção do elemento $e$ engloba os nós que estão localizados dentro de um subdomínio circular $\Omega_{e}$ de raio $r_{\min }$ e centralizado no centroide do elemento $e$, como mostra a Fig. 3.3(a). O valor de $r_{\min }$ deve ser independente da malha, ou seja, se a malha é refinada, a única diferença é a quantidade de nós que fazem influência no valor de $\gamma_{e}$, como mostra a Fig. 3.3(b).

Definidos os nós dentro do subdomínio do elemento e, é possível calcular a média ponderada $\mu_{e}$ das variáveis $d_{j}$ dentro de $\Omega_{e}$, dada por:

$$
\mu_{e}=\frac{\sum_{j \in \Omega_{e}} d_{j} w_{p}\left(\mathbf{x}_{j}-\mathbf{x}_{e}\right)}{\sum_{j \in \Omega_{e}} w_{p}\left(\mathbf{x}_{j}-\mathbf{x}_{e}\right)}
$$

onde $d_{j}$ é o conjunto de variáveis topológicas dentro da área de influência $\Omega_{e}$ do elemento $e$, e $w_{p}\left(\mathbf{x}_{j}-\mathbf{x}_{e}\right)$ é a função peso da projeção que calcula o quanto a variável $d_{j}$ influencia a pseudodensidade $\gamma_{e}$. Neste trabalho utiliza-se a função de projeção linear proposta por Bruns e Tortorelli (2001), dada por:

$$
w_{p}\left(\mathbf{x}_{j}-\mathbf{x}_{e}\right)=\left\{\begin{array}{cc}
\frac{r_{\min }-r_{j}}{r_{\min }} & \text { se } \mathbf{x} \in \Omega_{e} ; \\
0 & \text { caso contrário. }
\end{array}\right.
$$

sendo que $\Omega_{e}$ é definido como:

$$
\mathbf{x} \in \Omega_{e} \quad \text { se } \quad r_{j}=\left\|\mathbf{x}_{j}-\mathbf{x}_{e}\right\| \leq r_{\min }
$$

onde $r$ é a distância entre um nó arbitrário e o centroide do elemento $e, \mathbf{x}$ e $\mathbf{x}_{e}$ são suas respectivas coordenadas, e $r_{\min }$ é o raio da projeção. A Fig. 3.3 ilustra a técnica de projeção com a função peso $w_{p}$ linear, sendo que $r_{\text {min }}$ não depende da discretização da 
malha.

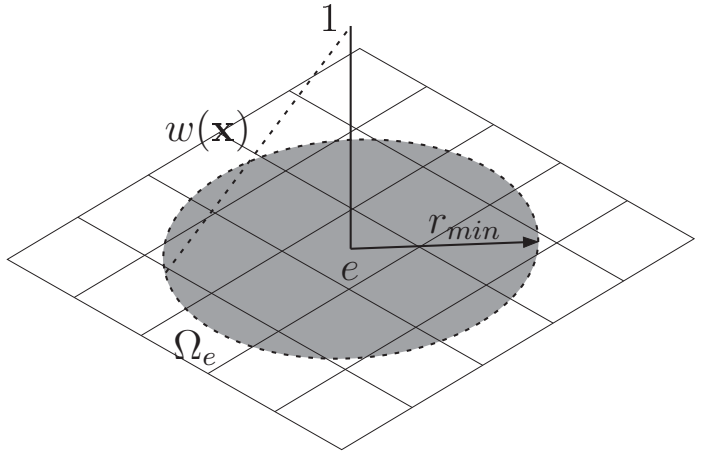

(a)

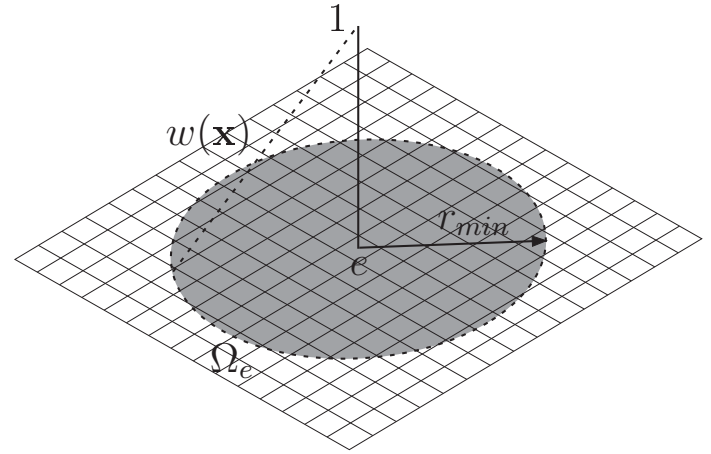

(b)

Figura 3.3: Representação da técnica de projeção.

Assim, é possível determinar o valor da pseudodensidade do elemento $\gamma_{e}$ utilizando a função "Heaviside" da seguinte maneira:

$$
\gamma_{e}=1-\mathrm{e}^{-\beta_{p} \mu_{e}}+\mu_{e} \mathrm{e}^{-\beta_{p}}
$$

onde $\beta_{p}$ que controla a curva da função exponencial e, consequentemente, penaliza os valores intermediários de $\mu_{e}$. A Fig. 3.4 mostra como o parâmetro $\beta_{p}$ influencia no valor de $\gamma_{e}$ em função do valor de $\mu_{e}$.

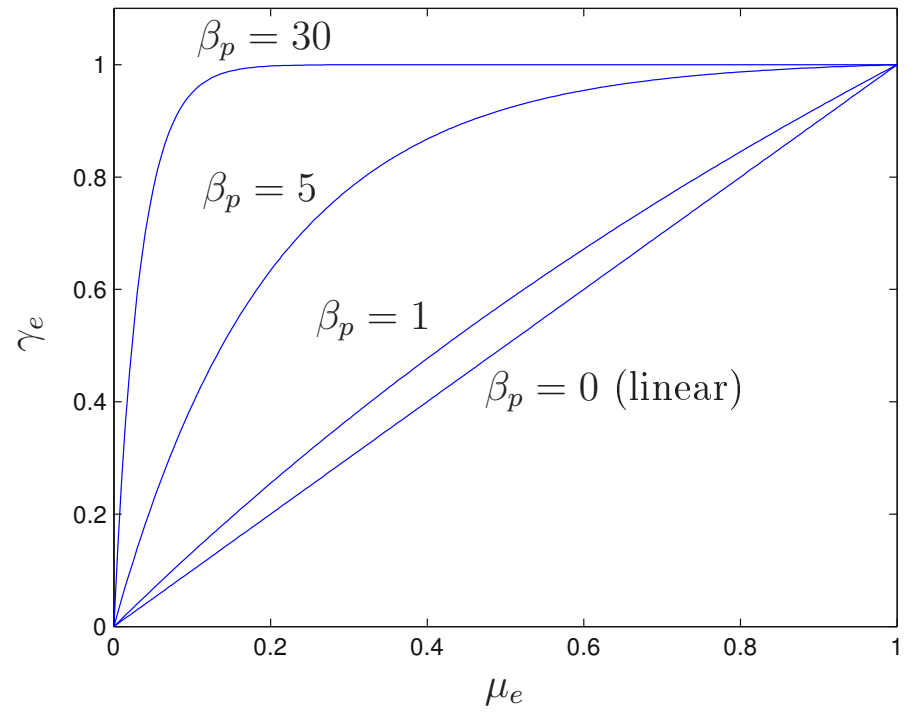

Figura 3.4: Função "Heaviside" para vários valores de $\beta_{p}$.

Para valores altos de $\beta_{p}$, por exemplo $\beta_{p}=30$, o problema se torna discreto, ou seja, não tem solução única. Assim, sugere-se utilizar o método da continuação onde $\beta_{p}$ começa com um valor baixo, por exemplo $\beta_{p}=1$ e aumenta gradativamente até que a solução não 
apresente valores intermediários de pseudo-densidades (GUEST; PREVOST; BELYTSCHKO, 2004).

Além disso, de acordo com o gráfico da Fig. 3.4, para que a pseudodensidade $\gamma_{e}$ se configure como um elemento vazio, seu valor deve ser igual a zero. Ou seja, pequenos valores de $\mu_{e}$ e altos valores de $\beta_{p}$ resultam em altos valores de $\gamma_{e}$, configurando elemento cheio (ou sólido). Entretanto, se o elemento for vazio, e consequentemente $\gamma_{e}=0$, as matrizes de rigidez, massa e amortecimento podem se tornar singulares, impossibilitando a obtenção dos deslocamentos. Assim, é necessário incluir um valor mínimo de $\gamma_{\min }$, geralmente igual a $\gamma_{\min }=10^{-6}$, para evitar essas singularidades numéricas. Dessa forma, as matrizes de rigidez e massa das Eqs. (3.15), (3.11) e (3.12) devem ser reescritas da seguinte maneira:

$$
\begin{aligned}
& \mathbf{K}_{\mathbf{u u}}^{e}=\left(\gamma_{e}^{p_{c}}+\gamma_{\text {min }}\right) \int_{\Omega^{e}} \mathbf{B}_{\mathbf{u}}^{T} \mathbf{T}_{\varepsilon}^{T} \mathbf{c}_{e f}^{E} \mathbf{T}_{\varepsilon} \mathbf{B}_{\mathbf{u}} d \Omega \\
& \mathbf{K}_{\phi \phi}^{e}=-\left(\gamma_{e}^{p_{\epsilon}}+\gamma_{\min }\right) \int_{\Omega^{e}} \mathbf{B}_{\phi}^{T} \mathbf{T}_{\phi}^{T} \boldsymbol{\epsilon} \mathbf{T}_{\phi} \mathbf{B}_{\phi} d \Omega \\
& \mathbf{M}^{e}=\left(\gamma_{e}^{p_{m}}+\gamma_{\min }\right) \rho_{0} \int_{\Omega^{e}} \mathbf{A}^{T} \mathbf{A} d \Omega
\end{aligned}
$$

lembrando que para elementos sólidos, o termo $\left(\gamma_{e}^{p}+\gamma_{\min }\right)$ deve respeitar o valor máximo igual a 1. A matriz de rigidez piezelétrica (Eq. (3.10)) não necessita da inclusão de $\gamma_{\text {min }}$ já que ela não faz parte da diagonal da matriz global de rigidez e se ela for totalmente nula, configura um elemento piezelétrico sem polarização.

Entretanto, utilizar técnicas de continuação aumenta o número de iterações e o resultado fica dependente do critério utilizado para aumentar o valor de $\beta_{p}$. Guest, Asadpoure e Ha (2011) propuseram algumas modificações no método para eliminar o método da continuação. A primeira modificação é no limite superior das variáveis de projeto $d$. Para que as pseudo-densidades $\gamma_{e}$ apresentem valores consistentes com a distribuição 0 e 1, ou seja, vazio ou sólido, as variáveis de projeto são usualmente restritas a valores entre 0 e 1 . Entretanto, utilizando o filtro proposto, as variáveis de projeto não tem sentido físico até que elas sejam projetadas para o cálculo das pseudo-densidades dos elementos $\gamma_{e}$ (GUEST; ASADPOURE; HA, 2011). Ao relaxar o valor máximo $d_{\max }$, a média ponderada $\mu_{e}$ também é relaxada, permitindo que o produto $\beta_{p} \mu_{e}$ da Eq. (3.20) tenha um valor alto. Entretanto, a função "degrau" deve ser modificada para que as pseudo-densidades permaneçam entre os limites 0 e 1. Assim, a Eq. (3.20) deve ser reescrita da seguinte maneira (GUEST; ASADPOURE; HA, 2011):

$$
\gamma_{e}=1-\mathrm{e}^{-\beta_{p} \mu_{e}}+\frac{\mu_{e}}{\mu_{\max }} \mathrm{e}^{-\beta_{p} \mu_{\max }}
$$


onde $\mu_{\max }=d_{\max }$. Utilizando essa nova formulação, é possível manter o valor de $\beta_{p}$ pequeno e constante. Neste trabalho, utiliza-se valores de $\beta_{p}$ e $d_{\max }$ propostos por Guest, Asadpoure e Ha (2011), ou seja, $\beta_{p}=2$ e $d_{\max }=100$. Além disso, Guest, Asadpoure e Ha (2011) propõe modificar o cálculo das assíntotas móveis das duas primeiras iterações do algoritmo "Method of Moving Asymptotes" (MMA) de modo que as primeiras assíntotas não fiquem muito longe das variáveis de projeto. Para as duas primeiras iterações da otimização, o MMA calcula as assíntotas da seguinte maneira (SVANBERG, 1987):

$$
\begin{aligned}
& l o w^{(k)}=d^{(k)}-0,5\left(d^{\text {max }}-d^{\text {min }}\right) \\
& u p p^{(k)}=d^{(k)}+0,5\left(d^{\text {max }}-d^{\text {min }}\right)
\end{aligned}
$$

onde $k$ é a iteração da rotina de otimização, low e upp são as assíntotas inferior e superior, e $d^{\text {max }}$ e $d^{\text {min }}$ são os limites que as variáveis de topologia podem assumir. O cálculo apresentado depende da diferença entre $d^{\text {max }}$ e $d^{\text {min }}$, que se for grande pode fazer com que as assíntotas fique muito distantes do valor das variáveis de topologia $d$, gerando muita oscilação na solução da rotina de otimização. Assim, Guest, Asadpoure e Ha (2011) propõe a seguinte modificação no algoritmo de MMA para as das primeiras iterações:

$$
\begin{aligned}
& l o w^{(k)}=d^{(k)}-\frac{0,5}{\beta_{p}+1} \\
& u p p^{(k)}=d^{(k)}+\frac{0,5}{\beta_{p}+1}
\end{aligned}
$$

Utilizando essa formulação, Guest, Asadpoure e Ha (2011) apresentam resultados de otimização em estruturas de vigas e mecanismos flexíveis com distribuição de material praticamente 0-1, ou seja, sem materiais intermediários gerados pela técnica de projeção de Bruns e Tortorelli (2001).

\subsection{Otimização Topológica com Restrição de Falha Mecânica}

Neste trabalho, a falha mecânica é baseada nos valores das tensões mecânicas calculadas na estrutura. Existem diversos critérios de falha mecânica, como por exemplo, critérios de tensão normal máxima, von Mises (BRUGGI; VENINI, 2008; LE et al., 2010), Tsai-Hill, Tsai-Wu (GROENWOLD; HAFTKA, 2006), critérios de delaminação para estruturas laminadas (JOHANSEN; LUND, 2009), dentre outros. Existem alguns trabalhos na literatura que utilizam alguns desses critérios como restrições de falha mecânica em problemas de otimização (GROENWOLD; HAFTKA, 2006; BRUGGI; VENINI, 
2008; JOHANSEN; LUND, 2009; LE et al., 2010), entretanto nenhum ainda aplicado a transdutores piezelétricos. Assim, deseja-se demonstrar neste trabalho a influência da restrição de falha mecânica no projeto otimizado de transdutores piezocompósitos.

O problema de otimização topológica incluindo o cálculo de tensões mecânicas apresenta algumas dificuldades que precisam ser contornadas para que o problema de OT seja solucionado com eficácia (BENDSøE; SIGMUND, 2003).

A primeira está relacionada com o fenômeno das topologias singulares, que consiste na degeneração do espaço de soluções (CHENG; JIANG, 1992; CHENG; GUO, 1997; ROZVANY, 2001). Ou seja, a solução ótima global geralmente faz parte de um espaço degenerado de soluções (CHENG; JIANG, 1992; KIRSCH, 1990), o que implica na incapacidade de algoritmos tradicionais de otimização, baseados nos gradientes, de atingir o ponto ótimo (STUMP, 2006; BRUGGI; VENINI, 2008). A Fig. 3.5 apresenta um exemplo de um domínio que apresenta um ponto ótimo singular. Para contornar esse problema, as tensões, calculadas em todo o domínio, devem ser relaxadas para eliminar as regiões degeneradas. Diversas técnicas de relaxação têm sido publicadas para tentar contornar esse problema (CHENG; GUO, 1997; DUYSINX; BENDSøE, 1998; BRUGGI, 2008).

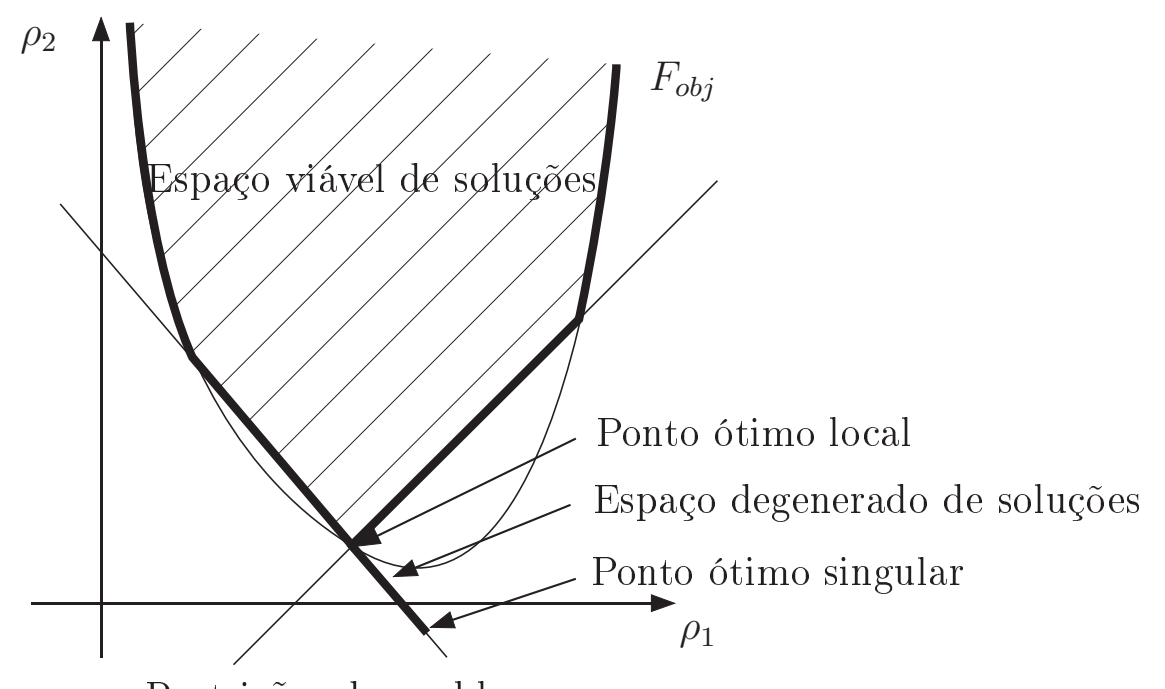

Restrições do problema

Figura 3.5: Representação genérica de um domínio de projeto que apresenta um ótimo singular.

A segunda dificuldade está relacionada com o fato de que a tensão mecânica é uma grandeza local, assim, as restrições devem ser consideradas para todos os pontos (LE et al., 2010). Num domínio discretizado, como é no caso da análise feita pelo MEF, o número de pontos em que as tensões são calculadas é finito, porém esse número pode ser relativamente grande o que implica num número de restrições que pode aumentar relativamente o tempo 
computacional, tanto para o cálculo das sensibilidades dessas restrições, quanto para a solução do problema de otimização por algoritmos tradicionais (STUMP, 2006; LE et al., 2010), como o MMA. Uma possível solução é adotar uma formulação de apenas uma restrição cujo valor se aproxima do valor de tensão máxima no domínio, como a função "norma-p" (DUYSINX; BENDSØE, 1998). Embora a restrição pela "norma-p" seja computacionalmente eficiente, ela não fornece um controle adequado da distribuição das tensões (LE et al., 2010), ou seja, pode haver regiões com alta concentração de tensão.

Além disso, como são utilizados diferentes tipos de materiais, o critério de falha também deve ser diferente. No caso de materiais isotrópicos, propõe-se utilizar o critério de tensão máxima de von Mises e compará-la com um certo limite de tensão. Para metais, é utilizado o limite de escoamento do material, e para cerâmicas piezelétricas é utilizado o limite de despolarização (CALDERON-MORENO, 2001), pois seu valor é bem inferior ao limite de ruptura da cerâmica. Já para materiais compósitos propõe-se, nesse trabalho, utilizar o critério de falha de Tsai-Wu. Este critério considera a interação entre as tensões nas diferentes direções com suas respectivas forças de resistência (HANSEL; BECKER, 1999). Para materiais ortotrópicos, são considerados apenas as tensões no plano da casca $\left(\sigma_{11}\right.$, $\sigma_{22}$ e $\tau_{12}$ ), e o critério avalia se a estrutura falha ou não, mas não distingue qual o modo de falha (longitudinal de tração ou compressão, transversal de tração ou compressão, ou cisalhamento) (HANSEL; BECKER, 1999; GROENWOLD; HAFTKA, 2006).

Assim, o problema quando se utiliza a tensão de von Mises juntamente com o critério de Tsai-Wu é que as grandezas dos valores são muito diferentes. Enquanto que as tensões de von Mises devem ser restritas na ordem de MPa, o índice de Tsai-Wu é adimensional e deve ter seu valor menor que 1 para não apresentar falhas. Essa grande diferença de valores causa também grande diferença nos valores das suas derivadas, o que pode atrapalhar na convergência da função objetivo.

Nesse aspecto, propõe-se utilizar o cálculo do fator de segurança como medida de normalização entre os diferentes critérios de tensão, já que ele é um valor adimensional e o menor valor admissível para o fator de segurança é igual a 1, que indica que os carregamentos aplicados estão no limite das tensões do material. O fator de segurança foi utilizado por Groenwold e Haftka (2006) para otimizar estruturas compósitas utilizando os critérios de Tsai-Wu e de Tsai-Hill. Os autores sugerem utilizar o valor do fator de segurança como função objetivo ao invés de utilizar diretamente os valores de Tsai-Wu e Tsai-Hill pois eles dependem do valor do carregamento aplicado. O valor do fator de segurança indica o número de vezes que o carregamento aplicado pode ser aumentado de modo que os limites de tensão considerados ainda sejam respeitados. Assim, Groenwold e 
Haftka (2006) maximizam esse valor de modo que o carregamento possa ser aumentado.

Portanto, propõe-se neste trabalho utilizar o fator de segurança como restrição do problema de otimização para qualquer tipo de material utilizado. A seguir é apresentado o método de relaxação utilizado para contornar a singularidade de tensões. Em seguida são apresentados os cálculos dos critérios de von Mises e Tsai-Wu, bem como o cálculo dos respectivos fatores de segurança.

\subsubsection{Relaxação das Tensões}

Dentre os tipos de formulação para relaxação das tensões, destacam-se a função relaxação- $\varepsilon$ (CHENG; GUO, 1997; ROZVANY; SOBIESZCZANSKI-SOBIESKI, 1992) e os modelos baseados no SIMP (DUYSINX; BENDSØE, 1998; BRUGGI, 2008; LE et al., 2010). Entretanto, esses autores mostram que ao utilizar o modelo baseado no SIMP para relaxar as tensões, a penalização das tensões deve ser menor que a penalização do modelo estrutural. Ou seja, considerando que a penalização da tensão é representado por $p_{\sigma}$ e a penalização estrutural é representado por $p_{c}$, então $p_{\sigma}<p_{c}$. Duysinx e Bendsøe (1998) também mostram que esse modelo, além de apresentar o mesmo nível de relaxação do modelo de material, evita a descontinuidade das tensões locais quando um elemento se torna vazio. Assim, este trabalho também utiliza o modelo de relaxação de tensões baseado no SIMP.

Então, seguindo a ideia de Le et al. (2010), o modelo de material para as matrizes de rigidez e massa continua o mesmo. Para calcular as tensões no elemento utiliza-se a Eq. (2.38) multiplicada pela pseudodensidade do elemento, elevado ao expoente $p_{\sigma}$ da seguinte maneira:

$$
\boldsymbol{\sigma}=\gamma_{e}^{p_{\sigma}} \mathbf{c}_{e f \sigma}^{E} \mathbf{T}_{\varepsilon} \mathbf{B}_{u} \mathbf{u}_{e}
$$

onde $\gamma_{e}$ é calculada pela função "degrau" da Eq. (3.20) e $\mathbf{c}_{e f_{\sigma}}^{E}$ é calculada pelo método OMD da seguinte forma:

$$
\mathbf{c}_{e f \sigma}^{E}=\sum_{i=1}^{n^{c}} w_{o_{i}} \mathbf{c}^{E} \mathbf{T}_{\theta_{i}}=w_{o_{1}} \mathbf{c}^{E} \mathbf{T}_{\theta_{1}}+w_{o_{2}} \mathbf{c}^{E} \mathbf{T}_{\theta_{2}}+\cdots+w_{o_{n} c} \mathbf{c}^{E} \mathbf{T}_{\theta_{n^{c}}}, \quad 0 \leq w_{o_{i}} \leq 1
$$

sendo que os coeficientes de peso $w_{o_{i}}$ são calculados da mesma maneira que na Eq. (3.16). 


\subsubsection{Critérios de von Mises e Tsai-Wu}

Neste trabalho utiliza-se as tensões de von Mises e de Tsai-Wu. A tensão de von Mises $\left(\sigma_{v m}\right)$ é calculada da seguinte maneira:

$$
\sigma_{v m}=\sqrt{\sigma_{11}^{2}+\sigma_{22}^{2}-\sigma_{11} \sigma_{22}+3\left(\tau_{12}^{2}+\tau_{23}^{2}+\tau_{13}^{2}\right)}
$$

que pode ser calculada na forma matricial como:

$$
\sigma_{v m}=\sqrt{\boldsymbol{\sigma}^{T} \mathbf{V} \boldsymbol{\sigma}}
$$

onde $\boldsymbol{\sigma}=\left\{\begin{array}{lllll}\sigma_{11} & \sigma_{22} & \tau_{12} & \tau_{23} & \tau_{13}\end{array}\right\}^{T}$ calculada pela Eq. (2.38), e

$$
\mathbf{V}=\left[\begin{array}{ccccc}
1 & -0.5 & 0 & 0 & 0 \\
-0.5 & 1 & 0 & 0 & 0 \\
0 & 0 & 3 & 0 & 0 \\
0 & 0 & 0 & 3 & 0 \\
0 & 0 & 0 & 0 & 3
\end{array}\right]
$$

Para elementos de casca assumindo estado plano de tensões, com materiais ortotrópicos, o índice de Tsai-Wu $\left(\sigma_{t w}\right)$ pode ser expresso como (GROENWOLD; HAFTKA, 2006)

$$
\sigma_{t w}=Y_{1} \sigma_{11}+Y_{2} \sigma_{22}+Y_{11} \sigma_{11}^{2}+Y_{22} \sigma_{22}^{2}+2 Y_{12} \sigma_{11} \sigma_{22}+Y_{66} \tau_{12}^{2}
$$

sendo que os coeficientes $Y_{1}, Y_{2}, Y_{11}, Y_{22}, Y_{12}$ e $Y_{66}$ estão relacionados com a resistência do material da seguinte maneira (GROENWOLD; HAFTKA, 2006):

$$
\begin{gathered}
Y_{1}=\frac{1}{T_{1}}-\frac{1}{C_{1}} \quad ; \quad Y_{2}=\frac{1}{T_{2}}-\frac{1}{C_{2}} \quad ; \quad Y_{11}=\frac{1}{T_{1} C_{1}} \\
Y_{11}=\frac{1}{T_{2} C_{2}} \quad ; \quad Y_{66}=\frac{1}{S^{2}} \quad ; \quad Y_{12}=-\frac{\sqrt{Y_{1} Y_{2}}}{2}
\end{gathered}
$$

onde $T_{1}$ e $C_{1}$ são as resistências à tração e compressão no sentido longitudinal da fibra, $T_{2}$ e $C_{2}$ são as resistências à tração e compressão no sentido transversal à fibra e $S$ é a resistência ao cisalhamento. É também possível expressar o índice de Tsai-Wu na forma matricial como:

$$
\sigma_{t w}=\overline{\boldsymbol{\sigma}}^{T} \mathbf{Y} \overline{\boldsymbol{\sigma}}
$$


onde $\overline{\boldsymbol{\sigma}}=\left\{\begin{array}{lllll}\sigma_{11} & \sigma_{22} & \tau_{12} & 1 & 1\end{array}\right\}^{T} \mathrm{e}$

$$
\mathbf{Y}=\left[\begin{array}{ccccc}
Y_{11} & Y_{12} & 0 & 0.5 Y_{1} & 0 \\
Y_{12} & Y_{22} & 0 & 0 & 0.5 Y_{2} \\
0 & 0 & Y_{66} & 0 & 0 \\
0.5 Y_{1} & 0 & 0 & 0 & 0 \\
0 & 0.5 Y_{2} & 0 & 0 & 0
\end{array}\right]
$$

\subsubsection{Restrição Global de Tensão}

No domínio de elementos finitos, seria necessário utilizar uma restrição para cada ponto onde as tensões são calculadas. Isso implicaria numa quantidade excessiva de restrições, tornando o problema muito custoso computacionalmente. Assim, Le et al. (2010) propõem utilizar somente o valor máximo da tensão no domínio como restrição. Essa abordagem reduz o custo computacional significativamente, porém, ela não garante um controle sobre a distribuição de tensões no domínio, podendo gerar áreas com altas concentrações de tensão. No entanto, nesse trabalho, essa abordagem já preenche os requisitos de limite de tensão pois não há a preocupação em controlar a distribuição de tensões.

Como mencionado anteriormente, para cada tipo de material é utilizado um certo critério para avaliar as tensões limites. A equação a seguir descreve as restrições.

$$
\begin{array}{ll}
\max _{g=1 \ldots n_{\sigma}}\left\{\sigma_{v m_{g}}\right\} \leq \sigma_{y}, & \text { para materiais istrópicos metálicos; } \\
\max _{g=1 \ldots n_{\sigma}}\left\{\sigma_{v m_{g}}\right\} \leq \sigma_{d}, & \text { para materiais cerâmicos piezelétricos; } \\
\max _{g=1 \ldots n_{\sigma}}\left\{\sigma_{t w_{g}}\right\}<1, & \text { para materiais ortotrópicos. }
\end{array}
$$

onde $\sigma_{y}$ e $\sigma_{d}$ são as tensões de limite de escoamento (do material isotrópico) e de limite de despolarização(do material piezelétrico), $g$ refere-se aos pontos onde as tensões são calculadas no domínio discretizado do MEF e $n_{\sigma}$ é o número total desses pontos. Para que o cálculo das tensões seja preciso, deve-se calculá-las nos pontos de Gauss da integração reduzida, ou seja, para um elemento de 8 nós, a integração reduzida é de 4 pontos de Gauss (COOK et al., 2007). Além disso, estruturas sujeitas a deformações de flexão tem a distribuição de tensões de acordo com a Fig. 3.6, onde os valores máximos de tensão são encontrados nas suas superfícies (TIMOSHENKO, 1984). Assim, propõe-se neste trabalho utilizar 8 pontos em cada elemento, sendo 4 na superfície inferior e 4 na superfície superior, ou seja, $n_{\sigma}=8 n_{e}$ onde $n_{e}$ é igual ao número de elementos. 


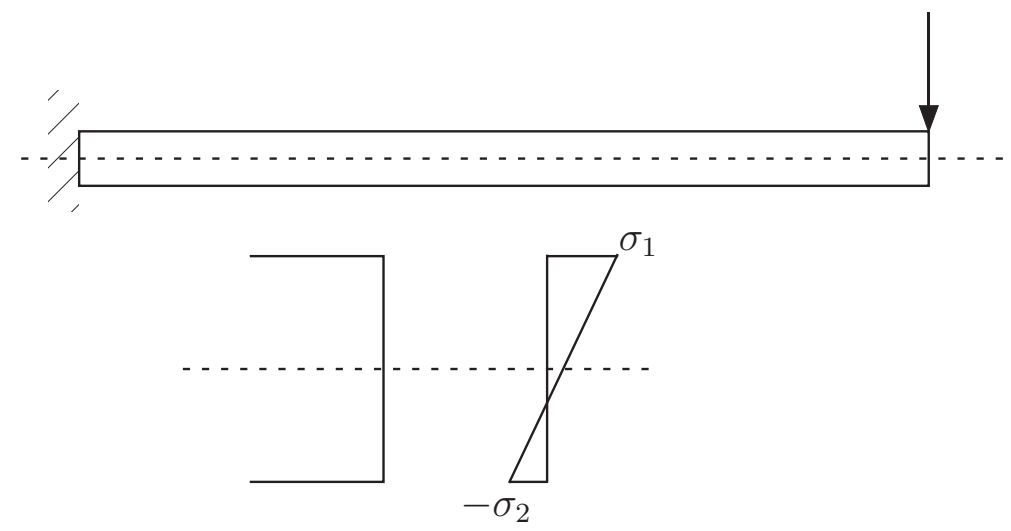

Figura 3.6: Distribuição de tensão ao longo da espessura em uma estrutura sujeita à flexão

Entretanto, utilizar três restrições com ordens de grandeza diferentes pode prejudicar a convergência do problema de otimização, já que os gradientes também terão grandezas diferentes. Assim, propõe-se utilizar uma única restrição da seguinte maneira:

$$
\min _{g=1 \ldots n_{\sigma}}\left\{s_{g}\right\} \geq 1
$$

onde $s_{g}$ é o fator de segurança calculado em cada ponto $g$ do domínio. Ou seja, o valor de $s_{g}$ para qualquer ponto, independente do tipo de material, deve ser maior ou igual a 1, o que muda entre os diferentes tipos de material é o modo como ele é calculado.

Para materiais isotrópicos, $s_{g}$ é calculado pela razão entre o limite de escoamento $\left(\sigma_{y}\right)$ e a tensão de von Mises. No caso das cerâmicas piezelétricas, calcula-se $s_{g}$ pela razão entre o limite de despolarização $\left(\sigma_{d}\right)$ e a tensão de von Mises. Ou seja, para esses tipos de materiais, pode-se resumir que $s_{g}$ é calculado pela razão entre a tensão limite do material $\left(\sigma_{\text {limite }}\right)$ e a tensão de von Mises. Assim, $s_{g}$ pode ser escrito como:

$$
s_{g}=\frac{\sigma_{\text {limite }}}{\sigma_{v m}}
$$

onde $\sigma_{\text {limite }}$ é igual ao limite de escoamento $\left(\sigma_{y}\right)$ para materiais isotrópicos ou o limite de despolarização $\left(\sigma_{d}\right)$ para materiais piezelétricos.

No caso de materiais ortotrópicos, $s_{g}$ é calculada como a solução de uma equação de segundo grau. Considerando que $s_{g}$ entra na Eq. (3.32) multiplicando as tensões, pode-se reescrever o critério de Tsai-Wu da seguinte maneira (GROENWOLD; HAFTKA, 2006):

$$
s_{g}^{2}\left(Y_{11} \sigma_{11}^{2}+Y_{22} \sigma_{22}^{2}+Y_{66} \tau_{12}^{2}+2 Y_{12} \sigma_{11} \sigma_{22}\right)+s_{g}\left(Y_{1} \sigma_{11}+Y_{2} \sigma_{22}\right)-1=0
$$


Assim, o fator de segurança para materiais ortotrópicos é obtido por:

$$
s_{g}=\frac{-b+\sqrt{b^{2}+4 a}}{2 a}
$$

onde

$$
\begin{gathered}
a=Y_{11} \sigma_{11}^{2}+Y_{22} \sigma_{22}^{2}+Y_{66} \tau_{12}^{2}+2 Y_{12} \sigma_{11} \sigma_{22} \\
b=Y_{1} \sigma_{11}+Y_{2} \sigma_{22}
\end{gathered}
$$

Os termos $a$ e $b$ podem ser convenientemente calculados matricialmente realizando-se a seguinte manipulação:

$$
\begin{aligned}
& a=\overline{\boldsymbol{\sigma}}^{T} \mathbf{Y}_{1} \overline{\boldsymbol{\sigma}} \\
& b=\overline{\boldsymbol{\sigma}}^{T} \mathbf{Y}_{2} \overline{\boldsymbol{\sigma}}
\end{aligned}
$$

onde

$$
\overline{\boldsymbol{\sigma}}=\left\{\begin{array}{c}
\sigma_{11} \\
\sigma_{22} \\
\tau_{12} \\
1 \\
1
\end{array}\right\} ; \quad \mathbf{Y}_{1}=\left[\begin{array}{ccccc}
Y_{11} & Y_{12} & 0 & 0 & 0 \\
Y_{12} & Y_{22} & 0 & 0 & 0 \\
0 & 0 & Y_{66} & 0 & 0 \\
0 & 0 & 0 & 0 & 0 \\
0 & 0 & 0 & 0 & 0
\end{array}\right] ; \quad \mathbf{Y}_{2}=\left[\begin{array}{ccccc}
0 & 0 & 0 & Y_{1} & 0 \\
0 & 0 & 0 & 0 & Y_{2} \\
0 & 0 & 0 & 0 & 0 \\
Y_{1} & 0 & 0 & 0 & 0 \\
0 & Y_{2} & 0 & 0 & 0
\end{array}\right]
$$

Entretanto a função max não é derivável. Uma alternativa para se encontrar o valor mínimo de um conjunto de valores é a função "norma-p" (LE et al., 2010). Assim, substitui-se a restrição da Eq. (3.37) por:

$$
s_{N P}=\left(\sum_{g=1}^{n_{\sigma}} s_{g}^{p_{n}}\right)^{1 / p_{n}} \geq 1
$$

sendo que $p_{n}<-1$ é a penalização utilizada para aproximar o valor de $s_{N P}$ do valor mínimo de $s_{g}$. Quanto maior for o módulo de $p_{n}$, mais próximo $s_{N P}$ será do valor mínimo de $s_{g}$.

Assim, tem-se uma única restrição global que atende os requisitos de tensão para cada tipo de material utilizado, cuja função seja derivável. 


\subsection{Implementação do MOT}

A metodologia do MOT proposta nesta tese segue basicamente o fluxograma apresentado na Fig. 3.7. Inicialmente é necessário entrar com os dados da malha como coordenadas dos nós, conectividade dos elementos, aplicação de forças (ou deslocamentos) e condições de contorno. Em seguida, as variáveis de projeto (de topologia, de polarização e de orientação) são inicializadas. Antes do laço de otimização, os nós são mapeados para encontrar os nós vizinhos de cada elemento para aplicar a técnica de projeção. Assim, dá-se início ao processo iterativo com a obtenção das pseudodensidades utilizando a técnica de projeção "Heaviside". Em seguida, são construídas as matrizes de rigidez, massa e amortecimento, para então obter os deslocamentos e potenciais elétricos solucionando-se o sistema do MEF. O próximo passo é calcular as funções objetivo e restrições para que se possa verificar a convergência e a viabilidade da solução. Na primeira iteração, a convergência não é verificada, passando para o cálculo das sensibilidades (derivadas) das funções objetivo e restrições com relação às variáveis de projeto. O último passo do laço de otimização é utilizar uma rotina de otimização para encontrar o mínimo da função objetivo respeitando as restrições impostas. A rotina de otimização utilizada neste trabalho é o MMA desenvolvido por Svanberg (1987) que utiliza a primeira derivada (análise de sensibilidades) das funções objetivo e das restrições e atualiza as variáveis de projeto conforme um dado critério. Esse processo é repetido iterativamente até que se observe a convergência da função objetivo. O software de otimização está implementado na linguagem do software comercial MATLAB (The Mathworks Inc., Natick, MA, USA) e para gerar a malha inicial de elementos finitos é utilizado o software comercial ANSYS (ANSYS Inc., Canonsburg, PA, USA).

Neste trabalho são utilizadas diferentes funções objetivo para cada tipo de aplicação, atuadores, sensores e coletores de energia. As funções e suas respectivas análises de sensibilidade são apresentadas nos capítulos individuais de cada projeto. Entretanto, as formulações apresentadas nesta seção são utilizadas para todos os projetos apresentados. Assim, é apresentada a análise de sensibilidades dessas formulações na seção a seguir.

\subsection{Análise de Sensibilidades das Formulações do MOT}

Para implementar o método de otimização proposto utilizando o algoritmo de otimização MMA (SVANBERG, 1987), é necessário realizar a análise de sensibilidades das funções objetivo e restrições do problema abordado. A análise de sensibilidades consiste 


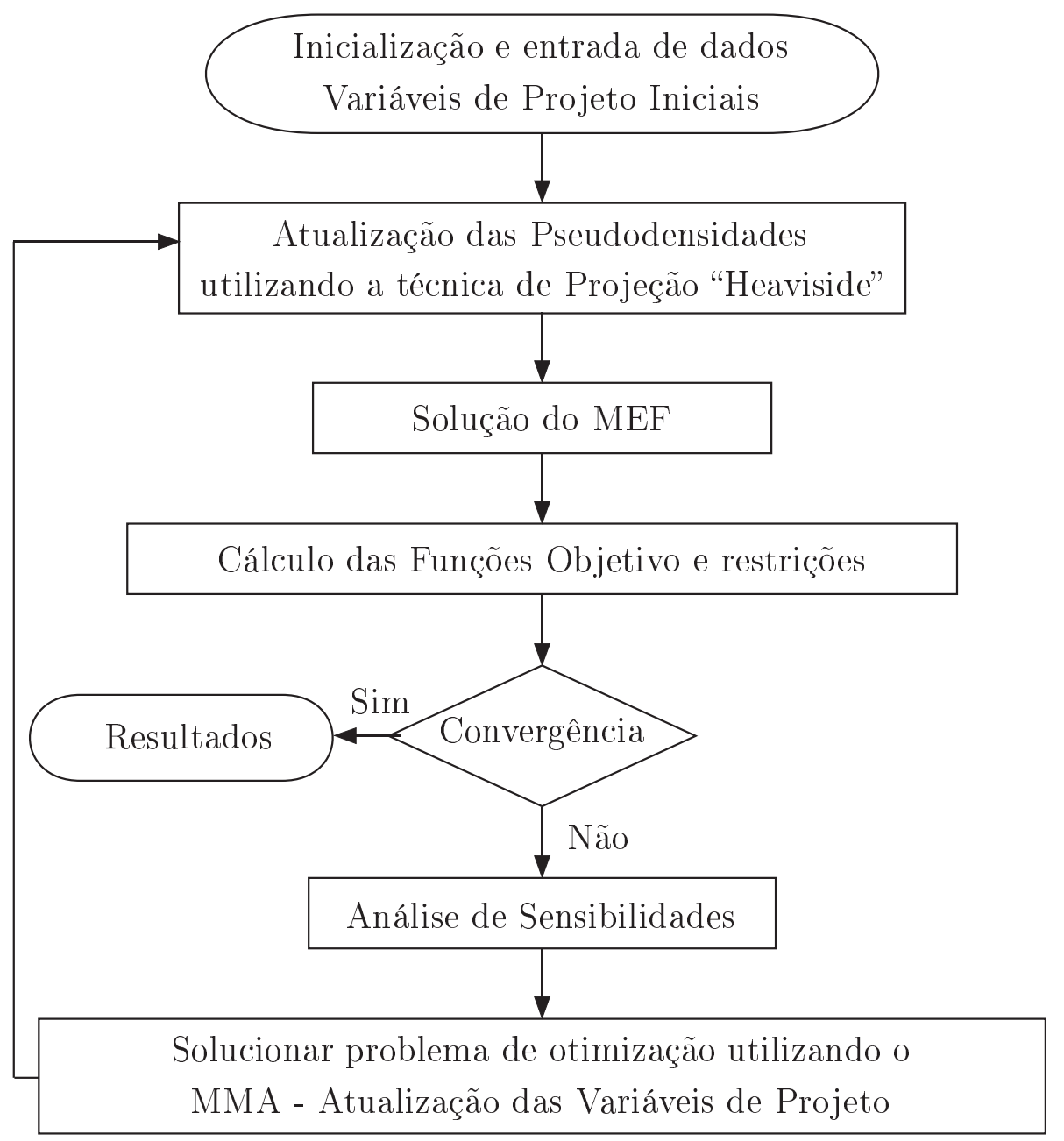

Figura 3.7: Fluxograma do procedimento de otimização implementado.

em calcular a primeira derivada de cada função objetivo e restrição em função das variáveis de projeto. Nesta tese propõe-se utilizar 3 diferentes variáveis de projeto: de topologia $d$, de polarização $\varrho$ e de orientação $\vartheta$.

As derivadas das funções objetivo para o projeto de atuadores, sensores e coletores de energia são apresentado nos seus respectivos capítulos. Na próxima seção, são apresentadas as derivadas das matrizes de rigidez e massa, da técnica de projeção e do método OMD e na seção seguinte é apresentada a derivada da restrição de falha mecânica.

\subsubsection{Derivadas das Matrizes de Rigidez e Massa}

As derivadas das matrizes de rigidez e massa são obtidas derivando-se as Eqs. (3.21), (3.22), (3.10) e (3.23). A derivada da matriz de amortecimento $\mathbf{C}$ é igual à soma das derivadas das matrizes $\mathbf{K}_{\mathbf{u u}}$ e $\mathbf{M}$, assim, não será demonstrado aqui. Derivando-as em 
relação às variáveis de topologia $d_{n}$, tem-se:

$$
\begin{aligned}
\frac{\partial \mathbf{K}_{\mathbf{u u}}^{e}}{\partial d_{n}} & =\left(p_{c} \gamma_{e}^{p_{c}-1} \frac{\partial \gamma_{e}}{\partial d_{n}}\right) \int_{\Omega^{e}} \mathbf{B}_{\mathbf{u}}^{T} \mathbf{T}_{\varepsilon}^{T} \mathbf{c}_{e f}^{E} \mathbf{T}_{\varepsilon} \mathbf{B}_{\mathbf{u}} d \Omega \\
\frac{\partial \mathbf{K}_{\mathbf{u} \phi}^{e}}{\partial d_{n}} & =\left(p_{e} \gamma_{e}^{p_{e}-1} \frac{\partial \gamma_{e}}{\partial d_{n}}\right)\left(2 \varrho_{e}-1\right)^{p_{i}} \int_{\Omega^{e}} \mathbf{B}_{\mathbf{u}}^{T} \mathbf{T}_{\varepsilon}^{T} \mathbf{e}_{0} \mathbf{T}_{\phi} \mathbf{B}_{\phi} d \Omega \\
\frac{\partial \mathbf{K}_{\phi \phi}^{e}}{\partial d_{n}} & =-\left(p_{\epsilon} \gamma_{e}^{p_{\epsilon}-1} \frac{\partial \gamma_{e}}{\partial d_{n}}\right) \int_{\Omega^{e}} \mathbf{B}_{\phi}^{T} \mathbf{T}_{\phi}^{T} \boldsymbol{\epsilon} \mathbf{T}_{\phi} \mathbf{B}_{\phi} d \Omega \\
\frac{\partial \mathbf{M}^{e}}{\partial d_{n}} & =\left(p_{m} \gamma_{e}^{p_{m}-1} \frac{\partial \gamma_{e}}{\partial d_{n}}\right) \rho_{0} \int_{\Omega^{e}} \mathbf{A}^{T} \mathbf{A} d \Omega
\end{aligned}
$$

pois somente $\gamma_{e}$ depende de $d_{n}$ e $n$ é o índice da variável topológica que está sendo analisada. Derivando as matrizes em relação à variável de polarização $\varrho_{e}$ somente a derivada da matriz piezelétrica $\mathbf{K}_{\mathbf{u} \phi}$ será diferente de zero, ou seja:

$$
\frac{\partial \mathbf{K}_{\mathbf{u} \phi}^{e}}{\partial \varrho_{e}}=\left(\gamma_{e}^{p_{e}}+\gamma_{\min }\right)\left[2 p_{i}\left(2 \varrho_{e}-1\right)^{p_{i}-1}\right] \int_{\Omega^{e}} \mathbf{B}_{\mathbf{u}}^{T} \mathbf{T}_{\varepsilon}^{T} \mathbf{e}_{0} \mathbf{T}_{\phi} \mathbf{B}_{\phi} d \Omega
$$

Com relação às variáveis de orientação $\vartheta_{i}^{e}$, apenas a matriz $\mathbf{K}_{\mathbf{u u}}^{e}$ apresenta derivada não nula, e pode ser calculada da seguinte maneira:

$$
\frac{\partial \mathbf{K}_{\mathbf{u u}}^{e}}{\partial \vartheta_{n}^{e}}=\left(\gamma_{e}^{p_{c}}+\gamma_{\min }\right) \int_{\Omega^{e}} \mathbf{B}_{\mathbf{u}}^{T} \mathbf{T}_{\varepsilon}^{T} \frac{\partial \mathbf{c}_{e f}^{E}}{\partial \vartheta_{n}^{e}} \mathbf{T}_{\varepsilon} \mathbf{B}_{\mathbf{u}} d \Omega
$$

Agora, é necessário calcular a sensibilidade das pseudodensidades $\gamma_{e}$ em função das variáveis topológicas $d_{n}$ derivando-se a técnica de projeção com a função "degrau". Portanto, derivando-se primeiramente a Eq. (3.24) pela regra da cadeia, tem-se:

$$
\frac{\partial \gamma_{e}}{\partial d_{n}}=\beta_{p} \mathrm{e}^{-\beta_{p} \mu_{e}} \frac{\partial \mu_{e}}{\partial d_{n}}+\frac{\mathrm{e}^{-\beta_{p} \mu_{\max }}}{\mu_{\max }} \frac{\partial \mu_{e}}{\partial d_{n}}
$$

onde $\partial \mu_{e} / \partial d_{n}$ é obtida derivando-se a função de projeção linear da Eq. (3.17), que resulta em:

$$
\frac{\partial \mu_{e}}{\partial d_{n}}=\frac{w_{p}\left(\mathbf{x}_{n}-\mathbf{x}_{e}\right)}{\sum_{j \in \Omega_{e}} w_{p}\left(\mathbf{x}_{j}-\mathbf{x}_{e}\right)}
$$

Finalmente, é necessário obter a sensibilidade do método OMD em função das variáveis de orientação $\vartheta_{n}^{e}$, que pode ser obtida calculando-se as derivadas das Eqs. (3.14) e (3.16), que pode ser escrita da seguinte forma:

$$
\frac{\partial \mathbf{c}_{e f}^{E}}{\partial \vartheta_{n}^{e}}=\sum_{i=1}^{n^{c}} \frac{\partial w_{o_{i}}}{\partial \vartheta_{n}^{e}} \mathbf{c}_{\theta_{i}}^{E}
$$


onde

$$
\frac{\partial w_{o_{i}}}{\partial \vartheta_{n}^{e}}=\frac{\partial \hat{w}_{i}}{\partial \vartheta_{n}^{e}} \frac{1}{\sum_{k=1}^{n^{c}} \hat{w}_{k}}-\frac{\hat{w}_{i}}{\left(\sum_{k=1}^{n^{c}} \hat{w}_{k}\right)^{2}} \sum_{k=1}^{n^{c}} \frac{\partial \hat{w}_{k}}{\partial \vartheta_{n}^{e}}
$$

sendo que

$$
\frac{\partial \hat{w}_{i}}{\partial \vartheta_{n}^{e}}=\left\{\begin{array}{cr}
p_{\vartheta}\left(\vartheta_{n}^{e}\right)^{p_{\vartheta}-1} \prod_{j=1, j \neq i}^{n^{c}}\left(1-\left(\vartheta_{j}^{e}\right)^{p_{\vartheta}}\right) & \text { se } n=i \\
-\left(\vartheta_{i}^{e}\right)^{p_{\vartheta}} p_{\vartheta}\left(\vartheta_{n}^{e}\right)^{p_{\vartheta}-1} \prod_{j=1, j \neq i, j \neq n}^{n^{c}}\left(1-\left(\vartheta_{j}^{e}\right)^{p_{\vartheta}}\right) & \text { se } n=j
\end{array}\right.
$$

\subsubsection{Derivada da Restrição de Falha Mecânica}

A sensibilidade da restrição de falha mecânica utilizada neste trabalho é obtida derivando-se a Eq. (3.46). Assim, com relação à uma variável genérica $v$, sua derivada fica:

$$
\frac{\partial s_{N P}}{\partial v}=\frac{1}{p_{n}}\left(\sum_{g=1}^{n_{\sigma}} s_{g}^{p_{n}}\right)^{\frac{1}{p_{n}}-1}\left[\sum_{g=1}^{n_{\sigma}} p_{n} s_{g}^{p_{n}-1} \frac{\partial s_{g}}{\partial v}\right]
$$

A derivada de $s_{g}$ depende do tipo de material onde se está calculando. Para materiais isotrópicos e piezelétricos, deriva-se a Eq. (3.38):

$$
\frac{\partial s_{g}}{\partial v}=-\frac{\sigma_{\text {limite }}}{\sigma_{v m}^{2}} \frac{\partial \sigma_{v m}}{\partial v}
$$

sendo que a derivada de $\sigma_{v m}$ é obtida derivando-se a Eq. (3.30), que fica da seguinte forma:

$$
\frac{\partial \sigma_{v m}}{\partial v}=\frac{1}{\sigma_{v m}}\left(\boldsymbol{\sigma}^{T} \mathbf{V} \frac{\partial \boldsymbol{\sigma}}{\partial v}\right)
$$

onde $\boldsymbol{\sigma}$ é o tensor de tensões. Substituindo a Eq. (3.60) na Eq. (3.59) a derivada do fator de segurança para materiais isotrópicos e piezelétricos fica:

$$
\frac{\partial s_{g}}{\partial v}=-\frac{\sigma_{\text {limite }}}{\sigma_{v m}^{3}} \boldsymbol{\sigma}^{T} \mathbf{V} \frac{\partial \boldsymbol{\sigma}}{\partial v}
$$

Para materiais ortotrópicos, derivando-se a Eq. (3.40), obtém-se:

$$
\frac{\partial s_{g}}{\partial v}=\frac{-\frac{\partial b}{\partial v}+\frac{1}{2 \sqrt{b^{2}+4 a}}\left(2 b \frac{\partial b}{\partial v}+4 \frac{\partial a}{\partial v}\right)}{2 a}-\frac{-b+\sqrt{b^{2}+4 a}}{4 a^{2}} 2 \frac{\partial a}{\partial v}
$$


que simplificando e agrupando os termos em $\frac{\partial a}{\partial v}$ e $\frac{\partial b}{\partial v}$ fica:

$$
\frac{\partial s_{g}}{\partial v}=\frac{1}{a}\left(\frac{1}{\sqrt{b^{2}+4 a}}-s_{g}\right) \frac{\partial a}{\partial v}+\frac{1}{2 a}\left(-1+\frac{b}{\sqrt{b^{2}+4 a}}\right) \frac{\partial b}{\partial v}
$$

onde $\frac{\partial a}{\partial v}$ e $\frac{\partial b}{\partial v}$ são obtidos derivando-se as Eqs. (3.43) e (3.44), respectivamente:

$$
\begin{aligned}
& \frac{\partial a}{\partial v}=2 \overline{\boldsymbol{\sigma}}^{T} \mathbf{Y}_{1} \frac{\partial \overline{\boldsymbol{\sigma}}}{\partial v} \\
& \frac{\partial b}{\partial v}=2 \overline{\boldsymbol{\sigma}}^{T} \mathbf{Y}_{2} \frac{\partial \overline{\boldsymbol{\sigma}}}{\partial v}
\end{aligned}
$$

sendo que

$$
\frac{\partial \overline{\boldsymbol{\sigma}}}{\partial v}=\left[\begin{array}{ccccc}
1 & 0 & 0 & 0 & 0 \\
0 & 1 & 0 & 0 & 0 \\
0 & 0 & 1 & 0 & 0 \\
0 & 0 & 0 & 0 & 0 \\
0 & 0 & 0 & 0 & 0
\end{array}\right]\left\{\begin{array}{c}
\sigma_{11} \\
\sigma_{22} \\
\tau_{12} \\
\tau_{13} \\
\tau_{23}
\end{array}\right\}=\mathbf{I}_{10} \frac{\partial \boldsymbol{\sigma}}{\partial v}
$$

Substituindo a Eq. (3.66) nas Eqs. (3.64) e (3.65), e depois substituindo-as na Eq. (3.67), a derivada de $s_{g}$ para materiais ortotrópicos fica:

$$
\frac{\partial s_{g}}{\partial v}=\left[\frac{2}{a}\left(\frac{1}{\sqrt{b^{2}+4 a}}-s_{g}\right) \overline{\boldsymbol{\sigma}}^{T} \mathbf{Y}_{1}+\frac{1}{a}\left(-1+\frac{b}{\sqrt{b^{2}+4 a}}\right) \overline{\boldsymbol{\sigma}}^{T} \mathbf{Y}_{2}\right] \mathbf{I}_{10} \frac{\partial \boldsymbol{\sigma}}{\partial v}
$$

Analisando as Eqs. (3.61) e (3.67), a derivada do fator de segurança pode ser escrito, de uma maneira genérica, como:

$$
\frac{\partial s_{g}}{\partial v}=\mathbf{S}_{m a t} \frac{\partial \boldsymbol{\sigma}}{\partial v}
$$

onde $\mathbf{S}_{m a t}$ é um vetor linha que depende do tipo de material que está analisando. Portanto, para materiais isotrópicos e piezelétricos, $\mathbf{S}_{m a t}$ é igual a

$$
\mathbf{S}_{\text {mat }}=-\frac{\sigma_{\text {limite }}}{\sigma_{v m}^{3}} \boldsymbol{\sigma}^{T} \mathbf{V}
$$

e para materiais ortotrópicos, $\mathbf{S}_{\text {mat }}$ é igual a

$$
\mathbf{S}_{m a t}=\left[\frac{2}{a}\left(\frac{1}{\sqrt{b^{2}+4 a}}-s_{g}\right) \overline{\boldsymbol{\sigma}}^{T} \mathbf{Y}_{1}+\frac{1}{a}\left(-1+\frac{b}{\sqrt{b^{2}+4 a}}\right) \overline{\boldsymbol{\sigma}}^{T} \mathbf{Y}_{2}\right] \mathbf{I}_{10}
$$

Chega-se finalmente à derivada do tensor de tensões $\boldsymbol{\sigma}$ em função da variável genérica $v$ que é obtida derivando-se a Eq. (3.27), cujos termos que dependem das variáveis de 
projeto são $\gamma_{e}, \mathbf{c}_{e f \sigma}^{E}$ e $\mathbf{u}_{e}$. Assim, aplicando a regra da cadeia, tem-se que:

$$
\frac{\partial \boldsymbol{\sigma}}{\partial v}=p_{\sigma} \gamma_{e}^{p_{\sigma}-1} \frac{\partial \gamma_{e}}{\partial v} \mathbf{c}_{e f \sigma}^{E} \mathbf{T}_{\varepsilon} \mathbf{B}_{u} \mathbf{u}_{e}+\gamma_{e}^{p_{\sigma}} \frac{\partial \mathbf{c}_{e f \sigma}^{E}}{\partial v} \mathbf{T}_{\varepsilon} \mathbf{B}_{u} \mathbf{u}_{e}+\gamma_{e}^{p_{\sigma}} \mathbf{c}_{e f \sigma}^{E} \mathbf{T}_{\varepsilon} \mathbf{B}_{u} \frac{\partial \mathbf{u}_{e}}{\partial v}
$$

Substituindo a Eq. (3.71) na Eq. (3.68) e posteriormente na Eq. (3.58), a derivada da função "norma-p" fica:

$$
\begin{array}{r}
\frac{\partial s_{N P}}{\partial v}=\left(\sum_{g=1}^{n_{\sigma}} s_{g}^{p_{n}}\right)^{\frac{1}{p_{n}}-1} \sum_{g=1}^{n_{\sigma}}\left\{s _ { g } ^ { p _ { n } - 1 } \mathbf { S } _ { m a t } \left[\left(p_{\sigma} \gamma_{e}^{p_{\sigma}-1} \frac{\partial \gamma_{e}}{\partial v} \mathbf{c}_{e f \sigma}^{E}+\right.\right.\right. \\
\left.\left.\left.\gamma_{e}^{p_{\sigma}} \frac{\partial \mathbf{c}_{e f \sigma}^{E}}{\partial v}\right) \mathbf{T}_{\varepsilon} \mathbf{B}_{u} \mathbf{u}_{e}+\gamma_{e}^{p_{\sigma}} \mathbf{c}_{e f \sigma}^{E} \mathbf{T}_{\varepsilon} \mathbf{B}_{u} \frac{\partial \mathbf{u}_{e}}{\partial v}\right]\right\}
\end{array}
$$

É possível separar os termos que multiplicam $\mathbf{u}_{e}$ dos termos que multiplicam $\partial \mathbf{u}_{e} / \partial v$. Assim, a Eq. (3.72) pode ser reescrita da seguinte forma:

$$
\frac{\partial s_{N P}}{\partial v}=\mathrm{Aux}_{1}+\mathrm{Aux}_{2}
$$

onde

$$
\begin{gathered}
\operatorname{Aux}_{1}=\left(\sum_{g=1}^{n_{\sigma}} s_{g}^{p_{n}}\right)^{\frac{1}{p_{n}}-1} \sum_{g=1}^{n_{\sigma}}\left[s_{g}^{p_{n}-1} \mathbf{S}_{m a t}\left(p_{\sigma} \gamma_{e}^{p_{\sigma}-1} \frac{\partial \gamma_{e}}{\partial v} \mathbf{c}_{e f \sigma}^{E}+\gamma_{e}^{p_{\sigma}} \frac{\partial \mathbf{c}_{e f \sigma}^{E}}{\partial v}\right) \mathbf{T}_{\varepsilon} \mathbf{B}_{u} \mathbf{u}_{e}\right] \\
\operatorname{Aux}_{2}=\left(\sum_{g=1}^{n_{\sigma}} s_{g}^{p_{n}}\right)^{\frac{1}{p_{n}}-1} \sum_{g=1}^{n_{\sigma}}\left(s_{g}^{p_{n}-1} \gamma_{e}^{p_{\sigma}} \mathbf{S}_{m a t} \mathbf{c}_{e f \sigma}^{E} \mathbf{T}_{\varepsilon} \mathbf{B}_{u} \frac{\partial \mathbf{u}_{e}}{\partial v}\right)
\end{gathered}
$$

O termo $A_{u x}$ é facilmente calculado em cada ponto $g$ da malha de elementos finitos, lembrando que $\frac{\partial \gamma_{e}}{\partial d_{n}}$ é calculada pelas Eqs. (3.53) e (3.54), e $\frac{\partial \mathbf{c}_{e f \sigma}^{E}}{\partial \vartheta_{n}^{e}}$ é calculada de maneira análoga à Eq. (3.55). Entretanto, o termo Aux 2 possui a derivada dos deslocamentos, que é um pouco mais complicado de ser calculado pois envolve inversão de matrizes.

Todavia, o termo Aux 2 pode ser devidamente rearranjado, de forma a ser calculado por uma multiplicação de dois vetores:

$$
\operatorname{Aux}_{2}=\mathbf{F}_{\sigma}^{T} \frac{\partial \mathbf{U}}{\partial v}
$$

onde a derivada do vetor global de deslocamentos $\mathbf{U}$ depende da forma como o MEF é resolvido, o que difere para cada tipo de aplicação apresentado nesta tese. Assim, $\frac{\partial \mathbf{U}}{\partial v}$ é descrito nos capítulos dos projetos de atuadores, sensores e coletores de energia. 


\section{PROJETO ESTÁTICO DE ATUADORES PIEZOCOMPÓSITOS DE CASCA}

Neste capítulo é apresentado o projeto otimizado de atuadores piezocompósitos de casca operando em regime estático. Os atuadores são capazes de gerar deslocamentos devido à aplicação de potencial elétrico nos eletrodos do material piezelétrico. Assim, a otimização topológica é aplicada para que, dada a aplicação de um potencial elétrico conhecido, o atuador possa gerar deslocamentos em determinados pontos de forma que a direção e sentido possam ser controlados. Deseja-se, então, maximizar os deslocamentos em determinados pontos gerados pela aplicação de um potencial elétrico entre os eletrodos do material piezelétrico otimizando-se a distribuição e o sentido de polarização do material piezelétrico e a orientação das fibra do material ortotrópico.

O capítulo está organizado da seguinte maneira: primeiramente é apresentado um breve histórico da aplicação de otimização topológica no projeto de atuadores piezocompósitos de placa e casca. Em seguida é apresentada uma modificação no sistema de equações do MEF para adaptar a problemas de atuadores excitados por potencial elétrico. Na continuação, o problema de otimização topológica para atuadores é formulado para o domínio contínuo e para o domínio discretizado. A próxima seção mostra o cálculo da sensibilidades da função objetivo com relação às variáveis de projeto. Na sequência, são apresentados alguns resultados numéricos para exemplificar o método. Finalmente, as conclusões desta parte do trabalho são apresentadas. 


\subsection{Histórico}

A aplicação de técnicas de otimização em projeto de atuadores de placa e casca não é recente (FRECKER, 2003; MUKHERJEE; JOSHI, 2002). Entretanto, o conceito de otimização topológica aplicado a esse tipo de atuadores foi introduzido por Kögl e Silva (2005) que aplicaram o MOT para a distribuição de material piezelétrico numa estrutura laminada juntamente com o sentido de sua polarização, com o objetivo de maximizar os deslocamentos em determinados pontos da estrutura.

Mais tarde, Kang e Tong (2008b) utilizaram o MOT para o projeto de estruturas laminadas através da distribuição de material piezelétrico e do valor das tensões elétricas ótimas para conseguir o desempenho desejado de seus atuadores. No entanto, Kang e Tong (2008a) apontam certas dificuldades de se aplicar um campo de tensões elétricas arbitrário num dado domínio. Assim, os mesmos autores apresentam novos resultados, considerando uma distribuição uniforme de tensões elétricas para um controle estático das deformações.

Howard et al. (2009) aplicam a OT de maneira similar ao trabalho de Kang e Tong (2008b), generalizando a aplicação tanto para estruturas de placa como para estruturas de casca.

Mais recentemente, Kang e Wang (2010) aplicam a OT em atuadores de placa multicamada cujo objetivo é obter um melhor controle dos deslocamentos.

\subsection{Excitação por Potencial Elétrico}

No projeto de atuadores piezocompósitos, o material piezelétrico é excitado aplicando-se uma diferença de potencial elétrico entre seus eletrodos. Como dito na Seção 2.3, a modelagem do MEF de casca piezelétrico utilizada neste trabalho considera dois tipos de grau de liberdade elétrico: um definido nos nós da superfície média da camada $\left(\bar{\phi}_{m}\right)$ e outro representando a ligação entre os eletrodos em cada camada $(\Delta \phi)$, os quais podem ser globalmente representados por $\boldsymbol{\Phi}_{m}$ e $\Delta \boldsymbol{\Phi}$, respectivamente. Assim, a Eq. (2.46), considerando somente regime estático $(\omega=0)$, pode ser reescrita da seguinte 
maneira:

$$
\left[\begin{array}{cccc}
\mathbf{K}_{\mathbf{u u}} & \mathbf{K}_{\mathbf{u} \phi_{1}} & \mathbf{K}_{\mathbf{u} \phi_{2}} & \overline{\mathbf{A}}^{T} \\
\mathbf{K}_{\mathbf{u} \phi_{1}}^{T} & \mathbf{K}_{\phi_{1} \phi_{1}} & \mathbf{K}_{\phi_{1} \phi_{2}} & \mathbf{0} \\
\mathbf{K}_{\mathbf{u} \phi_{2}}^{T} & \mathbf{K}_{\phi_{1} \phi_{2}}^{T} & \mathbf{K}_{\phi_{2} \phi_{2}} & \mathbf{0} \\
\overline{\mathbf{A}} & \mathbf{0} & \mathbf{0} & \mathbf{0}
\end{array}\right]\left\{\begin{array}{c}
\mathbf{U} \\
\boldsymbol{\Phi}_{m} \\
\Delta \boldsymbol{\Phi} \\
\boldsymbol{\lambda}
\end{array}\right\}=\left\{\begin{array}{c}
\mathbf{F} \\
\mathbf{Q} \\
\mathbf{P} \\
\mathbf{0}
\end{array}\right\}
$$

onde $\phi_{1}$ e $\phi_{2}$ representam os graus de liberdade $\bar{\phi}_{m}$ e $\Delta \phi$, respectivamente, e $\mathbf{Q}$ e $\mathbf{P}$ representam as respectivas cargas elétricas internas (dos nós) e do eletrodo. Entretanto, as cargas elétricas com respeito aos graus de liberdade elétricos internos são iguais a zero, ou seja, $\mathbf{Q}=\mathbf{0}$.

Quando somente potencial elétrico $\Delta \boldsymbol{\Phi}$ é aplicado entre os eletrodos, a resposta da estrutura é calculada prescrevendo-se os graus de liberdade de potencial elétrico e assume-se que não há força aplicadas na estrutura, ou seja, $\mathbf{F}=\mathbf{0}$. Assim, a Eq. (4.1) pode ser simplificada como:

$$
\left[\begin{array}{ccc}
\mathbf{K}_{\mathbf{u u}} & \mathbf{K}_{\mathbf{u} \phi_{1}} & \overline{\mathbf{A}}^{T} \\
\mathbf{K}_{\mathbf{u} \phi_{1}}^{T} & \mathbf{K}_{\phi_{1} \phi_{1}} & \mathbf{0} \\
\overline{\mathbf{A}} & \mathbf{0} & \mathbf{0}
\end{array}\right]\left\{\begin{array}{c}
\mathbf{U} \\
\boldsymbol{\Phi}_{m} \\
\boldsymbol{\lambda}
\end{array}\right\}=-\left[\begin{array}{c}
\mathbf{K}_{\mathbf{u} \phi_{2}} \\
\mathbf{K}_{\phi_{1} \phi_{2}} \\
\mathbf{0}
\end{array}\right] \Delta \boldsymbol{\Phi} \text { ou } \hat{\mathbf{K}} \hat{\mathbf{U}}=\hat{\mathbf{F}}
$$

sendo que esse sistema é resolvido pelo Matlab.

\subsection{Formulação do Problema de OT para Atuadores no Domínio Contínuo}

Considerando-se que $\hat{\mathbf{U}}_{1}$ são os deslocamentos da estrutura gerados pela aplicação de potencial elétrico $\Delta \phi_{1}$ nos eletrodos do material piezelétrico (representado por $\Gamma_{\phi}$ ), o objetivo da otimização é maximizar os deslocamentos $\hat{\mathbf{U}}_{1_{a}}$ numa certa região desejada $\Gamma_{f_{a}}$, seguindo a direção e sentido de uma força auxiliar unitária $\mathbf{f}_{1_{a}}$. A direção e sentido de $\mathbf{f}_{1_{a}}$ são dados de entrada do problema e, portanto, podem ser controlados pelo projetista. A Fig. 4.1 representa um domínio de projeto $\Gamma_{\Omega}$ de um atuador piezocompósito, onde a sequência de camadas, de baixo para cima, representa os materiais isotrópico, ortotrópico (com fibras representadas pelas retas pontilhadas) e piezelétrico, respectivamente.

Assim, a função objetivo relacionada à maximização dos deslocamentos $\hat{\mathbf{U}}_{1_{a}}$ em $\Gamma_{f_{a}}$ na direção $\mathbf{f}_{1_{a}}$ pode ser escrita da seguinte maneira:

$$
L_{1}=\hat{\mathbf{U}}_{1_{a}}=\int_{\Gamma_{f a}} \mathbf{f}_{1_{a}}^{T} \hat{\mathbf{U}}_{1} d \Gamma_{\Omega}
$$




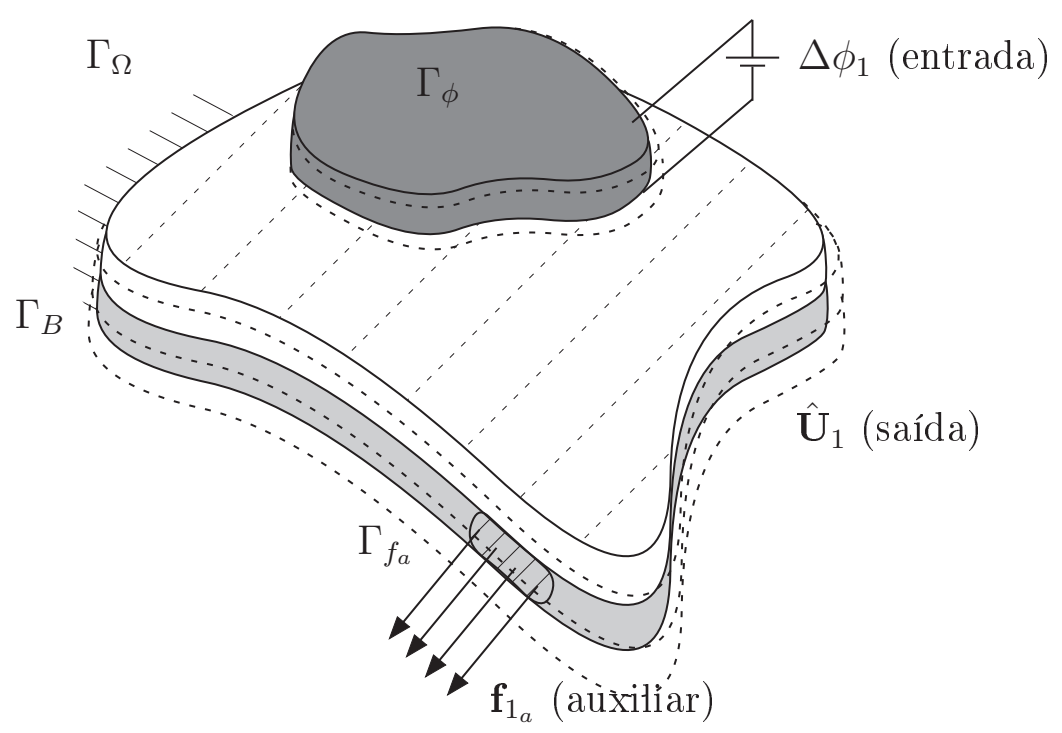

Figura 4.1: Representação do domínio de projeto de um atuador piezocompósito. Os contornos tracejados representam os deslocamentos $\hat{\mathbf{U}}_{1}$ devido à aplicação de potencial elétrico $\Delta \phi_{1}$.

Entretanto, a ordem de grandeza do valor de $L_{1}$ é igual a ordem de grandeza de $\hat{\mathbf{U}}_{1_{a}}$. Svanberg (1987) sugere que o módulo do valor da função objetivo utilizada esteja entre 1 e 100 para que o algoritmo MMA retorne valores razoáveis das variáveis de projeto. Assim, propõe-se neste trabalho, utilizar a seguinte função objetivo para o problema de otimização:

$$
£_{o b j}=\ln L_{1}
$$

que normaliza o valor de $L_{1}$. Caso sejam obtidos valores negativos de $L_{1}$ nas iterações iniciais, uma função objetivo alternativa é utilizada, já que a função ln não permite valores negativos. A função alternativa pode ser escrita como:

$$
£_{o b j}=\frac{1}{L_{1}}
$$

que é utilizada até que $L_{1}$ seja maior ou igual a 0, e então a função da Eq. (4.4) passa a ser utilizada.

No domínio contínuo, a distribuição de material, a polarização do material piezelétrico e a orientação das fibras do material ortotrópico são parametrizadas pelas pseudodensidades $\gamma$, pelas variáveis de polarização $\varrho$ e pelos ângulos $\boldsymbol{\theta}$, respectivamente. Portanto, o problema de otimização para atuadores piezocompósitos pode ser formulado, 
no domínio contínuo, da seguinte maneira:

$$
\begin{array}{cl}
\underset{\boldsymbol{\gamma}, \boldsymbol{\varrho}, \boldsymbol{\theta}}{\operatorname{Maximizar}} & £_{o b j} \\
\text { sujeito à: } & \text { Equações de equilíbrio } \\
& 0 \leq \boldsymbol{\gamma}(\mathbf{x}) \leq 1 \\
& 0 \leq \boldsymbol{\varrho}(\mathbf{x}) \leq 1 \\
& -90^{\circ} \leq \boldsymbol{\theta}(\mathbf{x}) \leq 90^{\circ}
\end{array}
$$

Deve-se lembrar que as equações de equilíbrio estão sujeitas às equações de acoplamento entre camadas, descrita na Seção 2.7. Neste trabalho, está sendo adotada uma formulação de otimização topológica sem restrição de volume, como pode ser observado na Eq. (4.6). Assim, é possível obter o volume ótimo de material piezelétrico que deve ser utilizado para maximizar os deslocamentos desejados.

Vale ressaltar que a função objetivo aqui proposta funciona para o problema de otimização topológica onde sempre existe uma camada de substrato que não está sujeita a alterações na topologia. Caso contrário, deve-se utilizar uma função para garantir uma certa rigidez na estrutura, como a função de "Mean Transduction" (SILVA; FONSECA; KIKUCHI, 1997; SILVA et al., 1999; CARBONARI; SILVA; NISHIWAKI, 2005), para evitar uma solução onde se tenha grandes deslocamentos mas com uma estrutura vazia.

\subsection{Formulação do Problema de OT para Atuadores no Domínio Discretizado}

Para implementar o MOT de acordo com o fluxograma apresentado na Seção 3.4 é necessário transformar o problema de otimização, apresentado anteriormente, do domínio contínuo para o discretizado. Primeiramente, discretiza-se a função $L_{1}$ da seguinte maneira:

$$
L_{1}=\mathbf{F}_{1}^{T} \hat{\mathbf{U}}_{1}
$$

onde $\mathbf{F}_{1}$ (que tem o mesmo tamanho de $\hat{\mathbf{U}}_{1}$ ) é o vetor global de força auxiliar que contém valor igual a 1 somente nos graus de liberdade em que se deseja maximizar os deslocamentos e valor nulo nos graus de liberdade restantes, e $\hat{\mathbf{U}}_{1}$ é o vetor global de deslocamentos obtido a partir da solução do sistema linear da Eq. (4.2).

Agora no domínio discretizado, a distribuição de material e a orientação das fibras são discretizadas pelas variáveis topológicas $\mathbf{d}$ de acordo com a técnica de projeção, e 
pelas variáveis de orientação $\vartheta$ de acordo com a OMD. A polarização continua sendo representada pela variável de polarização @. Assim, o problema de otimização para atuadores piezocompósitos pode ser formulado, no domínio discretizado, da seguinte maneira:

$$
\begin{array}{cl}
\underset{\mathbf{d}, \boldsymbol{\varrho}, \boldsymbol{\vartheta}}{\operatorname{Maximizar}:} & £_{o b j} \\
\text { sujeito à: } & \hat{\mathbf{K}} \hat{\mathbf{U}}=\hat{\mathbf{F}} \\
& 0 \leq \mathbf{d} \leq 1 \\
& 0 \leq \boldsymbol{\varrho} \leq 1 \\
& 0 \leq \boldsymbol{\vartheta} \leq 1
\end{array}
$$

\subsection{Análise de Sensibilidades}

Como dito na Seção 3.4, a rotina de otimização MMA utiliza a primeira derivada da função objetivo e das restrições para encontrar os valores ótimos das variáveis de projeto a cada iteração. Assim, neste caso de atuadores, é necessário somente obter a primeira derivada da função objetivo, já que não são utilizadas restrições no problema. Assim derivando-se a função objetivo $£_{o b j}$ (Eq. (4.4)) em função de uma variável genérica $v$, tem-se:

$$
\frac{\partial £}{\partial v}=\frac{1}{L_{1}} \frac{\partial L_{1}}{\partial v}
$$

onde a variável $v$ é utilizada para simplificar o desenvolvimento das derivadas, sendo que, posteriormente, ela deve ser substituída por $d, \varrho$ ou $\vartheta$. Para valores negativos de $L_{1}$, deve-se derivar a Eq. (4.5) que fica:

$$
\frac{\partial £}{\partial v}=-\frac{1}{L_{1}^{2}} \frac{\partial L_{1}}{\partial v}
$$

O próximo passo é derivar a função $L_{1}$ da Eq. (4.7):

$$
\frac{\partial L_{1}}{\partial v}=\mathbf{F}_{1}^{T} \frac{\partial \hat{\mathbf{U}}_{1}}{\partial v}
$$

considerando que $\frac{\partial \mathbf{F}_{1}}{\partial v}=\mathbf{0}$ já que $\mathbf{F}_{1}$ não depende das variáveis de projeto.

Em seguida, para obter a derivada do vetor de deslocamentos $\hat{\mathbf{U}}_{1}$, deve-se derivar a 
Eq. (4.2) com relação à uma variável genérica $v$, resultando em:

$$
\left[\begin{array}{ccc}
\frac{\partial \mathbf{K}_{\mathbf{u u}}}{\partial v} & \frac{\partial \mathbf{K}_{\mathbf{u} \phi_{1}}}{\partial v} & \mathbf{0} \\
\frac{\partial \mathbf{K}_{\mathbf{u} \phi_{1}}^{T}}{\partial v} & \frac{\partial \mathbf{K}_{\phi_{1} \phi_{1}}}{\partial v} & \mathbf{0} \\
\mathbf{0} & \mathbf{0} & \mathbf{0}
\end{array}\right]\left\{\begin{array}{c}
\mathbf{U}_{1} \\
\boldsymbol{\Phi}_{m 1} \\
\boldsymbol{\lambda}_{1}
\end{array}\right\}+\left[\begin{array}{ccc}
\mathbf{K}_{\mathbf{u u}} & \mathbf{K}_{\mathbf{u} \phi_{1}} & \overline{\mathbf{A}}^{T} \\
\mathbf{K}_{\mathbf{u} \phi_{1}}^{T} & \mathbf{K}_{\phi_{1} \phi_{1}} & \mathbf{0} \\
\overline{\mathbf{A}} & \mathbf{0} & \mathbf{0}
\end{array}\right]\left\{\begin{array}{c}
\frac{\partial \mathbf{U}_{1}}{\partial v} \\
\frac{\partial \boldsymbol{\Phi}_{m 1}}{\partial v} \\
\mathbf{0}
\end{array}\right\}=-\left[\begin{array}{c}
\frac{\partial \mathbf{K}_{\mathbf{u} \phi_{2}}}{\partial v} \\
\frac{\partial \mathbf{K}_{\phi_{1} \phi_{2}}}{\partial v} \\
\mathbf{0}
\end{array}\right] \Delta \boldsymbol{\Phi}(4.12)
$$

Como o potencial elétrico $\Delta \boldsymbol{\Phi}_{1}$ é prescrito, sua derivada com relação às variáveis de projeto é nula. Além disso, $\boldsymbol{\lambda}_{1}$ não depende das variáveis de projeto, o que resulta em derivada nula. Isolando do lado esquerdo o termo que multiplica a derivada dos deslocamentos, tem-se:

$$
\left[\begin{array}{ccc}
\mathbf{K}_{\mathbf{u u}} & \mathbf{K}_{\mathbf{u} \phi_{1}} & \overline{\mathbf{A}}^{T} \\
\mathbf{K}_{\mathbf{u} \phi_{1}}^{T} & \mathbf{K}_{\phi_{1} \phi_{1}} & \mathbf{0} \\
\overline{\mathbf{A}} & \mathbf{0} & \mathbf{0}
\end{array}\right]\left\{\begin{array}{c}
\frac{\partial \mathbf{U}_{1}}{\partial v} \\
\frac{\partial \boldsymbol{\Phi}_{m 1}}{\partial v} \\
\mathbf{0}
\end{array}\right\}=-\left[\begin{array}{ccc}
\frac{\partial \mathbf{K}_{\mathbf{u u}}}{\partial v} & \frac{\partial \mathbf{K}_{\mathbf{u} \phi_{1}}}{\partial v} & \mathbf{0} \\
\frac{\partial \mathbf{K}_{\mathbf{u} \phi_{1}}}{\partial v} & \frac{\partial \mathbf{K}_{\phi_{1} \phi_{1}}}{\partial v} & \mathbf{0} \\
\mathbf{0} & \mathbf{0} & \mathbf{0}
\end{array}\right]\left\{\begin{array}{c}
\mathbf{U}_{1} \\
\boldsymbol{\Phi}_{m 1} \\
\boldsymbol{\lambda}_{1}
\end{array}\right\}-\left[\begin{array}{c}
\frac{\partial \mathbf{K}_{\mathbf{u} \phi_{2}}}{\partial v} \\
\frac{\partial \mathbf{K}_{\phi_{1} \phi_{2}}}{\partial v} \\
\mathbf{0}
\end{array}\right] \Delta \boldsymbol{\Phi}(4
$$

No lado direito, o termo $\boldsymbol{\lambda}_{1}$ multiplica somente zeros e pode ser removido da equação. Pode-se ainda, juntar os dois termos do lado direito desta equação. Assim, a Eq. (4.13) pode ser reescrita da seguinte maneira:

$$
\left[\begin{array}{ccc}
\mathbf{K}_{\mathbf{u u}} & \mathbf{K}_{\mathbf{u} \phi_{1}} & \overline{\mathbf{A}}^{T} \\
\mathbf{K}_{\mathbf{u} \phi_{1}}^{T} & \mathbf{K}_{\phi_{1} \phi_{1}} & \mathbf{0} \\
\overline{\mathbf{A}} & \mathbf{0} & \mathbf{0}
\end{array}\right]\left\{\begin{array}{c}
\frac{\partial \mathbf{U}_{1}}{\partial v} \\
\frac{\partial \mathbf{\Phi}_{m_{1}}}{\partial v} \\
\mathbf{0}
\end{array}\right\}=-\left[\begin{array}{ccc}
\frac{\partial \mathbf{K}_{\mathbf{u u}}}{\partial v} & \frac{\partial \mathbf{K}_{\mathbf{u} \phi_{1}}}{\partial v} & \frac{\partial \mathbf{K}_{\mathbf{u} \phi_{2}}}{\partial v} \\
\frac{\partial \mathbf{K}_{\mathbf{u} \phi_{1}}}{\partial v} & \frac{\partial \mathbf{K}_{\phi_{1} \phi_{1}}}{\partial v} & \frac{\partial \mathbf{K}_{\phi_{1} \phi_{2}}}{\partial v} \\
\mathbf{0} & \mathbf{0} & \mathbf{0}
\end{array}\right]\left\{\begin{array}{c}
\mathbf{U}_{1} \\
\boldsymbol{\Phi}_{m 1} \\
\Delta \boldsymbol{\Phi}_{1}
\end{array}\right\}
$$

Finalmente, isolando a derivada do vetor de deslocamentos, chega-se a:

$$
\left\{\begin{array}{c}
\frac{\partial \mathbf{U}_{1}}{\partial v} \\
\frac{\partial \mathbf{\Phi}_{m_{1}}}{\partial v} \\
\mathbf{0}
\end{array}\right\}=-\left[\begin{array}{ccc}
\mathbf{K}_{\mathbf{u u}} & \mathbf{K}_{\mathbf{u} \phi_{1}} & \overline{\mathbf{A}}^{T} \\
\mathbf{K}_{\mathbf{u} \phi_{1}}^{T} & \mathbf{K}_{\phi_{1} \phi_{1}} & \mathbf{0} \\
\overline{\mathbf{A}} & \mathbf{0} & \mathbf{0}
\end{array}\right]^{-1}\left[\begin{array}{ccc}
\frac{\partial \mathbf{K}_{\mathbf{u u}}}{\partial v} & \frac{\partial \mathbf{K}_{\mathbf{u} \phi_{1}}}{\partial v} & \frac{\partial \mathbf{K}_{\mathbf{u} \phi_{2}}}{\partial v} \\
\frac{\partial \mathbf{K}_{\mathbf{u} \phi_{1}}^{T}}{\partial v} & \frac{\partial \mathbf{K}_{\phi_{1} \phi_{1}}}{\partial v} & \frac{\partial \mathbf{K}_{\phi_{1} \phi_{2}}}{\partial v} \\
\mathbf{0} & \mathbf{0} & \mathbf{0}
\end{array}\right]\left\{\begin{array}{c}
\mathbf{U}_{1} \\
\boldsymbol{\Phi}_{m 1} \\
\Delta \boldsymbol{\Phi}_{1}
\end{array}\right\}
$$

De acordo com a Eq. (4.15) é necessário realizar uma inversão da matriz de rigidez global para cada variável de projeto, o que resulta num alto custo computacional, inviabilizando o problema. Para contornar essa situação, é utilizado o método adjunto que calcula a derivada da função objetivo diretamente, ao invés de calcular a derivada de $\hat{\mathbf{U}}_{1}$ para cada variável de projeto. 
Assim, substituindo Eq. (4.15) na Eq. (4.9), a derivada da função objetivo fica:

$$
\frac{\partial L_{1}}{\partial v}=-\mathbf{F}_{1}^{T}\left[\begin{array}{ccc}
\mathbf{K}_{\mathbf{u u}} & \mathbf{K}_{\mathbf{u} \phi_{1}} & \overline{\mathbf{A}}^{T} \\
\mathbf{K}_{\mathbf{u} \phi_{1}}^{T} & \mathbf{K}_{\phi_{1} \phi_{1}} & \mathbf{0} \\
\overline{\mathbf{A}} & \mathbf{0} & \mathbf{0}
\end{array}\right]^{-1}\left[\begin{array}{ccc}
\frac{\partial \mathbf{K}_{\mathbf{u u}}}{\partial v} & \frac{\partial \mathbf{K}_{\mathbf{u} \phi_{1}}}{\partial v} & \frac{\partial \mathbf{K}_{\mathbf{u} \phi_{2}}}{\partial v} \\
\frac{\partial \mathbf{K}_{\mathbf{u} \phi_{1}}^{T}}{\partial v} & \frac{\partial \mathbf{K}_{\phi_{1} \phi_{1}}}{\partial v} & \frac{\partial \mathbf{K}_{\phi_{1} \phi_{2}}}{\partial v} \\
\mathbf{0} & \mathbf{0} & \mathbf{0}
\end{array}\right]\left\{\begin{array}{c}
\mathbf{U}_{1} \\
\boldsymbol{\Phi}_{m 1} \\
\Delta \boldsymbol{\Phi}_{1}
\end{array}\right\}
$$

O método adjunto se aplica substituindo o termo

$$
\mathbf{F}_{1}^{T}\left[\begin{array}{ccc}
\mathbf{K}_{\mathbf{u u}} & \mathbf{K}_{\mathbf{u} \phi_{1}} & \overline{\mathbf{A}}^{T} \\
\mathbf{K}_{\mathbf{u} \phi_{1}}^{T} & \mathbf{K}_{\phi_{1} \phi_{1}} & \mathbf{0} \\
\overline{\mathbf{A}} & \mathbf{0} & \mathbf{0}
\end{array}\right]^{-1}
$$

pela solução de um sistema linear na forma

$$
\left[\begin{array}{ccc}
\mathbf{K}_{\mathbf{u u}} & \mathbf{K}_{\mathbf{u} \phi_{1}} & \overline{\mathbf{A}}^{T} \\
\mathbf{K}_{\mathbf{u} \phi_{1}}^{T} & \mathbf{K}_{\phi_{1} \phi_{1}} & \mathbf{0} \\
\overline{\mathbf{A}} & \mathbf{0} & \mathbf{0}
\end{array}\right] \hat{\mathbf{U}}_{1_{a d j}}=\mathbf{F}_{1}
$$

resultando em

$$
\frac{\partial L_{1}}{\partial v}=-\hat{\mathbf{U}}_{1_{a d j}}\left[\begin{array}{ccc}
\frac{\partial \mathbf{K}_{\mathbf{u u}}}{\partial v} & \frac{\partial \mathbf{K}_{\mathbf{u} \phi_{1}}}{\partial v} & \frac{\partial \mathbf{K}_{\mathbf{u} \phi_{2}}}{\partial v} \\
\frac{\partial \mathbf{K}_{\mathbf{u} \phi_{1}}^{T}}{\partial v} & \frac{\partial \mathbf{K}_{\phi_{1} \phi_{1}}}{\partial v} & \frac{\partial \mathbf{K}_{\phi_{1} \phi_{2}}}{\partial v} \\
\mathbf{0} & \mathbf{0} & \mathbf{0}
\end{array}\right]\left\{\begin{array}{c}
\mathbf{U}_{1} \\
\boldsymbol{\Phi}_{m 1} \\
\Delta \boldsymbol{\Phi}_{1}
\end{array}\right\}
$$

Como $\hat{\mathbf{U}}_{1_{\text {adj }}}$ não possui termos com derivadas, ele pode ser calculado apenas uma vez a cada iteração, reduzindo o custo computacional.

\subsection{Resultados}

Nesta seção, são apresentados resultados numéricos de dois exemplos para o projeto otimizado de atuadores piezocompósitos de casca utilizando a metodologia proposta. $\mathrm{O}$ primeiro exemplo utiliza uma estrutura curva que representa um quarto de cilindro e o segundo exemplo utiliza uma estrutura de placa quadrada. Para cada exemplo, são estudados três casos de otimização para demonstrar a metodologia proposta aplicada a projeto de atuadores:

caso 1: substrato de alumínio coberto por duas camadas de material piezelétrico, considerando otimização de distribuição e de polarização do material piezelétrico;

caso 2: substrato composto de uma camada de fibra de carbono entre duas camadas 
de alumínio, coberto por duas camadas de material piezelétrico, considerando otimização de distribuição e de polarização do material piezelétrico e otimização de orientação das fibras;

caso 3: caso 2 com agrupamento de fibras.

A Fig. 4.2 ajuda a ilustrar os três casos. Para os três casos, as camadas de substrato e piezelétricas têm espessura iguais a $t_{s}=0,4 \mathrm{~mm}$ e $t_{p}=0,2 \mathrm{~mm}$, respectivamente. Nos casos 2 e 3, o substrato é dividido em três camadas, duas de alumínio com uma de fibra de carbono entre elas e suas espessuras são iguais a $t_{a}=0,05 \mathrm{~mm}$ e $t_{c}=0,3$ $\mathrm{mm}$, respectivamente, ou seja, a espessura do substrato continua com 0,4 mm. Nesses dois casos, a camada de alumínio serve como eletrodo para as camadas piezelétricas, já que a camada de fibra de carbono não é condutora de eletricidade. Para os dois exemplos apresentados, as propriedades dos materiais utilizados podem ser encontrados no Apêndice C sendo que, neste trabalho, utiliza-se o material piezelétrico utilizado da Tab. C.3.

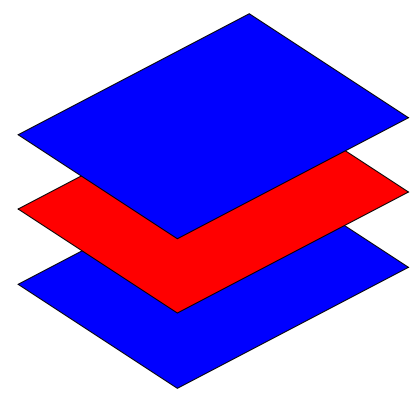

(a) Caso 1

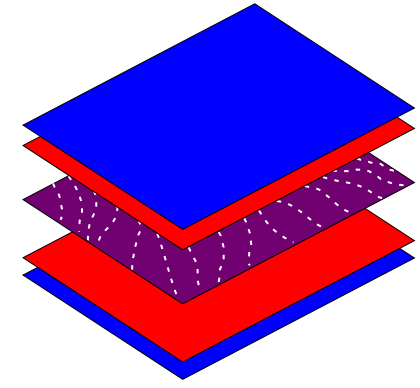

(b) Caso 2

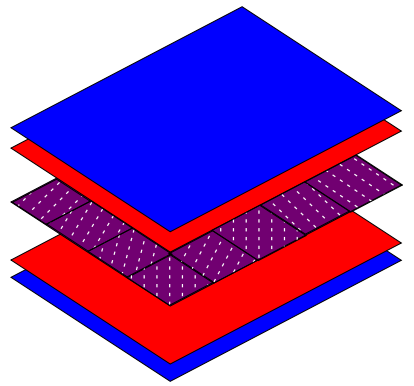

(c) Caso 3

Figura 4.2: Representação do empilhamento de camadas para o exemplo de atuadores de placa. A mesma sequência é utilizada para o exemplo de casca. As cores azul, vermelho e roxo, indicam as camadas de material piezelétrico, de alumínio e de fibra de carbono, respectivamente.

As otimizações de distribuição de material e de sentido de polarização são aplicadas somente às camadas de material piezelétrico para verificar sua influência no valor do deslocamento desejado. As camadas de substrato não sofrem alteração da topologia, somente de orientação de fibras no caso da fibra de carbono. Essa abordagem é adotada para evitar a utilização de outra função objetivo para minimizar a flexibilidade da estrutura, como a função de flexibilidade média (SILVA et al., 1999; SILVA; NISHIWAKI; KIKUCHI, 2000; CARBONARI; SILVA; NISHIWAKI, 2005, 2007). Esse tipo de função funciona, 
basicamente, minimizando os deslocamentos na estrutura, o que prejudicaria a eficácia da função de maximização de deslocamentos.

Para a otimização de orientação dos ângulos de fibras no caso 2, são adotados 12

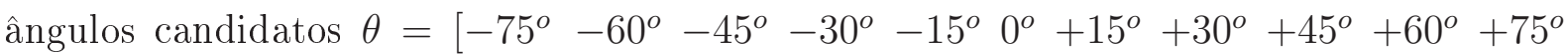
$+90^{\circ}$. Neste trabalho, atribui-se, inicialmente, valores iguais para todas as variáveis de topologia $d=0,5$ e de polarização $\varrho=0,6$. Não se utiliza o valor $\varrho=0,5$ para não gerar polarização nula logo no início do processo de otimização e criar instabilidades numéricas no método. O valor inicial para as variáveis de orientação deve ser igual a 0,5 de modo que nenhum ângulo candidato seja favorecido no início do processo de otimização. Para todos os exemplos apresentados, foram realizadas várias análises utilizando diferentes valores para as variáveis iniciais, que no final de cada análise, resultou em praticamente os mesmos resultados com valores de deslocamento muito próximos, com diferenças de no máximo 2\%. Assim, é possível afirmar que para os exemplos apresentados, chegou-se a soluções ótimas que estão muito próximas ao ótimo global.

São aplicados potenciais elétricos de $100 \mathrm{~V}$ e $-100 \mathrm{~V}$ nos eletrodos das camadas de cima e de baixo, respectivamente, conforme Fig. 4.3(a), de modo a simular uma ligação em paralelo dos eletrodos numa aplicação real, conforme Fig. 4.3(b).

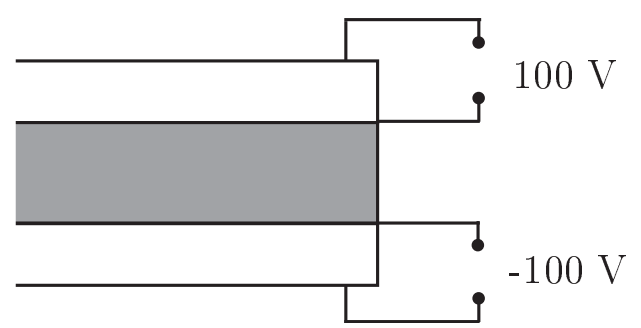

(a) Eletrodos no modelo de elementos finitos

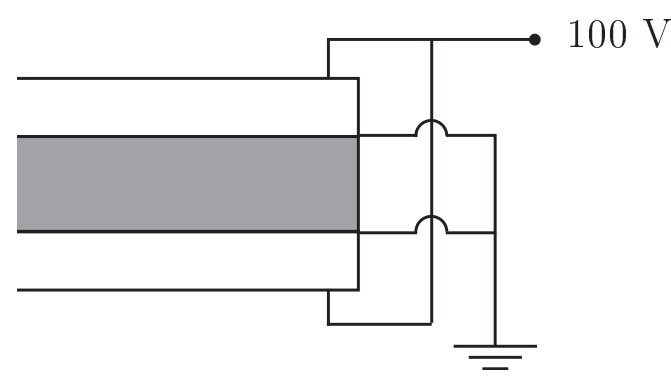

(b) Interpretação dos eletrodos para uma aplicação real

Figura 4.3: Esquema dos eletrodos para aplicação de potencial elétrico.

Nas figuras de distribuição de material, são apresentados os valores das pseudodensidades, onde a cor preta representa o elemento cheio e a cor branca representa o elemento vazio. Valores intermediários das pseudodensidades são apresentadas em escalas de cinza. Nas figuras de polarização, os elementos em preto e branco possuem polarização positiva e negativa, respectivamente. Nas figuras de otimização de orientação, são apresentadas as orientações ótimas em cada elemento por meio de uma reta. Nas figuras das deformadas, as camadas em verde e azul representam as camadas piezelétricas 
com polarização negativa e positiva, respectivamente, e as camadas em vermelho e roxo representam as camadas de alumínio e de fibra de carbono, respectivamente.

Finalmente, os valores dos coeficientes de penalização do modelo PEMAP-P são baseados nos trabalhos de Kögl e Silva (2005). Assim, $p_{\epsilon}=p_{i}=p_{m}=1, p_{c}=3 \mathrm{e}$ $p_{e}=4$. Entretanto, para evitar que a solução fique "presa" num mínimo local por causa dos expoentes $p_{c}$ e $p_{e}$, utiliza-se o método da continuação nesses valores. Inicialmente, $p_{c}=p_{e}=1$. Nas iterações 10, 20 e 30, $p_{e}$ é incrementado em uma unidade, e nas iterações 30 e 40, $p_{c}$ é incrementado em uma unidade. Para o coeficiente de penalização da orientação $p_{\vartheta}$ também é utilizada a técnica da continuação como proposto por Stegmann e Lund (2005a). Neste trabalho, inicialmente $p_{\vartheta}=1$, e tem seu valor aumentado em uma unidade nas iterações 10, 20, 30, 40 e 50, finalizando com valor igual a 6 .

\subsubsection{Exemplo 1}

Neste exemplo, é utilizado um domínio de um quarto de cilindro, conforme apresentado na Fig. 4.4. O comprimento da estrutura é igual a $l=15 \mathrm{~mm}$ e o raio interno é igual a $r_{i}=20 \mathrm{~mm}$. O raio utilizado na técnica de projeção é igual a $2 \mathrm{~mm}$. Os ângulos das orientações das fibras são também representados na Fig. 4.4 onde o ângulo $0^{\circ}$ segue a direção circunferencial e $90^{\circ}$ segue o eixo z. O sentido de polarização do material piezelétrico segue a direção radial, sendo que a polarização positiva segue o sentido de dentro para fora do cilindro.

Os retângulos em cinza representam os planos nos quais a estrutura está engastada, ou seja, os deslocamentos e rotações são nulos $\left(u_{x}=u_{y}=u_{z}=0\right.$ e $\left.\alpha=\beta=0\right)$. Entretanto, somente a camada de substrato está engastada, ou seja, a camada de material piezelétrico está apenas colada sobre o substrato e não é presa no engaste. Essa abordagem aproxima a modelagem de uma estrutura real, já que a fixação geralmente é feita apenas no substrato.

Foi realizada uma análise de convergência da malha de elementos finitos e foi constatada convergência nas análises quando a estrutura é dividida em 30 elementos na direção circunferencial e 15 elementos na direção do eixo $z$ em cada camada. Não foi utilizada uma malha mais refinada pois o elemento de casca utilizado, junto com o método OMD são muito custosos computacionalmente.

A Fig. 4.5 apresenta a forma deformada da estrutura sem aplicar nenhuma otimização, ou seja, as camadas de material piezelétrico são totalmente cheias e com polarização negativa para gerar deslocamento positivo em y no ponto A. Esse caso será chamado de 


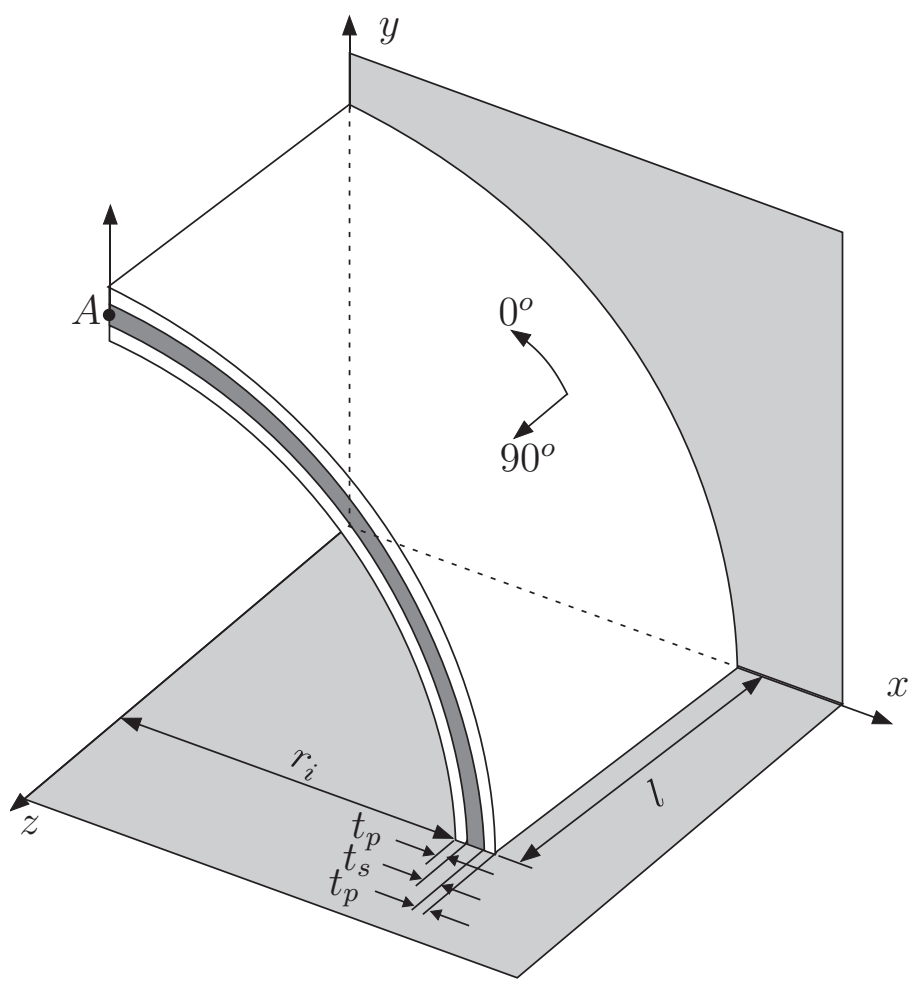

Figura 4.4: Domínio de projeto para o exemplo 1 de atuador. Medidas em mm. caso 0 , e obteve-se um deslocamento de $16,67 \mu \mathrm{m}$.

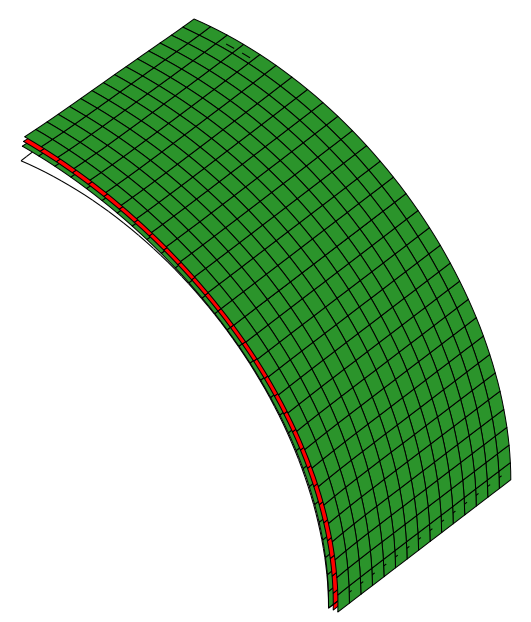

Figura 4.5: Forma deformada da estrutura para o caso 0 do atuador de casca, ou seja, sem otimização, com as camadas piezelétricas totalmente cheias e com polarização negativa.

As Figs. 4.6 e 4.7 referem-se aos resultados de otimização de topologia e de polarização para o caso 1 e a Fig. 4.8 apresenta sua respectiva deformada. O valor de deslocamento obtido neste caso é de $22,36 \mu \mathrm{m}, 34,13 \%$ maior que no caso 0. Nota-se pela Fig. 4.6 que não é necessário que as camadas piezelétricas estejam totalmente cheias sendo que os volumes ótimos de material piezelétrico para as camadas interna e externa são iguais a $93,28 \%$ e $88,57 \%$, respectivamente. Entretanto, o fator que realmente contribui para 
a maximização do deslocamento no ponto $A$ é a diferença de polarização numa mesma camada como apresentado na Fig. 4.7.

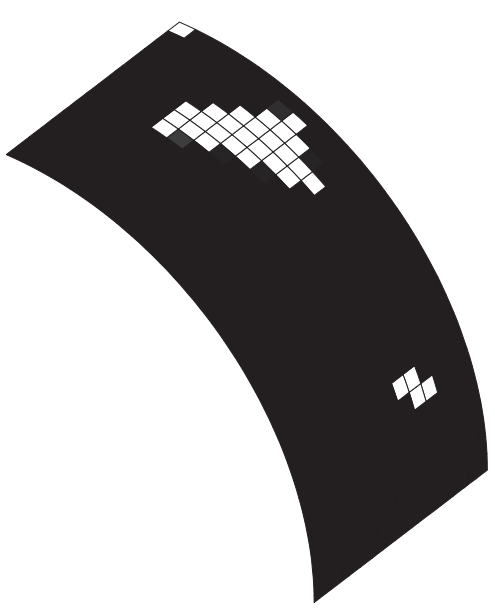

(a) Camada piezelétrica interna

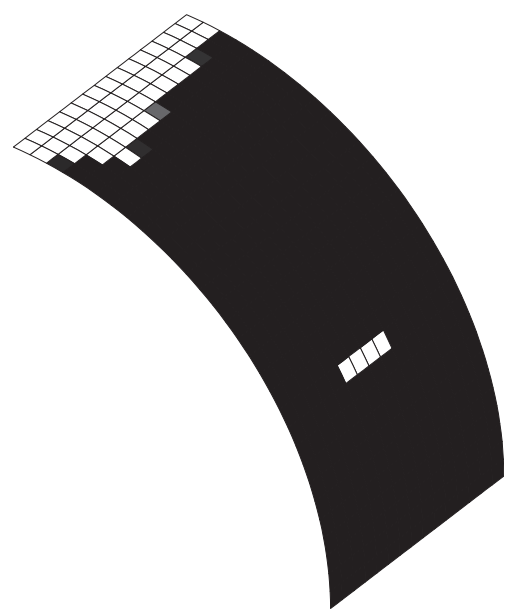

(b) Camada piezelétrica externa

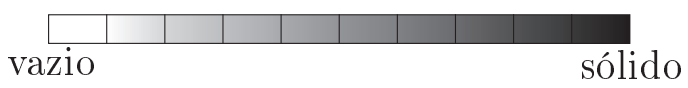

Figura 4.6: Resultado da otimização de topologia das camadas piezelétricas para o caso 1 de atuadores de casca.

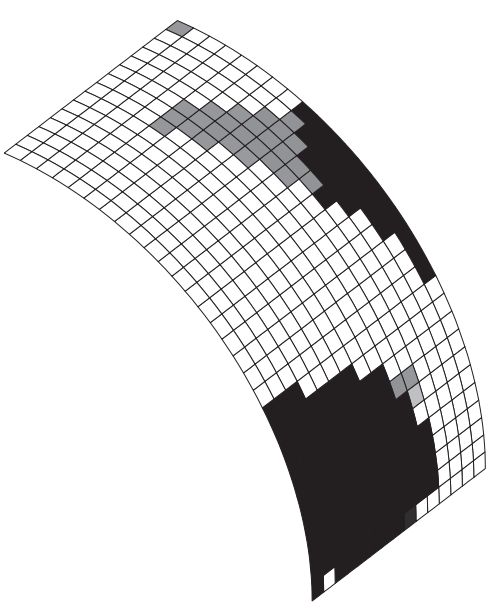

(a) Camada piezelétrica interna

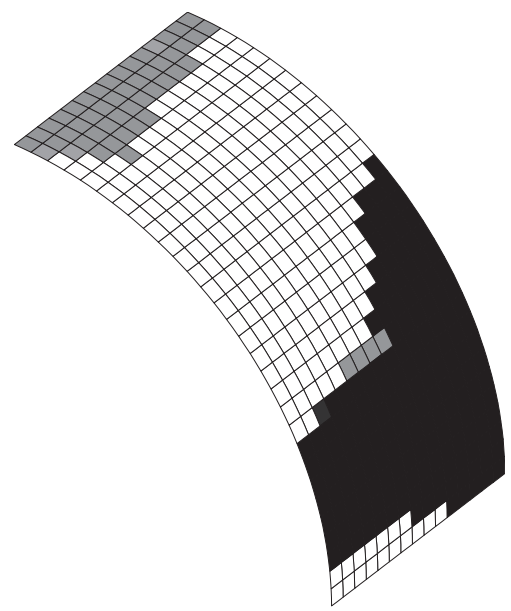

(b) Camada piezelétrica externa

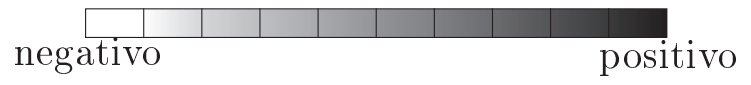

Figura 4.7: Resultado da otimização de polarização das camadas piezelétricas para o caso 1 de atuadores de casca. 


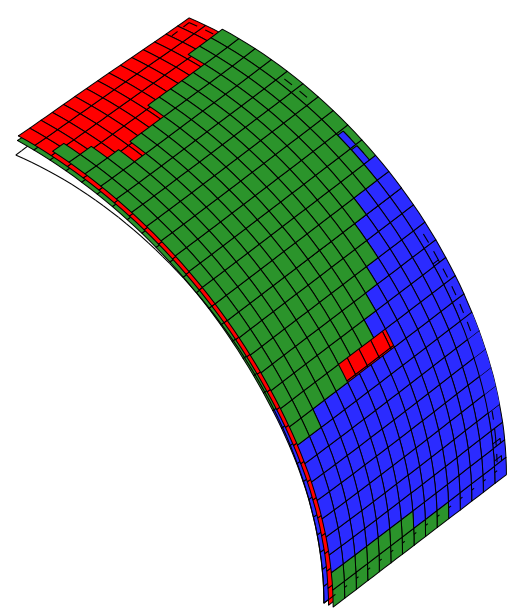

Figura 4.8: Forma deformada da estrutura para o caso 1 de atuadores de casca. Em vermelho está representada a camada de alumínio, e em azul e verde está representada a camada piezelétrica com polarização positiva e negativa, respectivamente.

Na Fig. 4.9 são apresentadas as distribuições de tensão mecânica nas superfícies inferior e superior somente das camadas de material piezelétrico, pois são as camadas que apresentam valores de tensão mais próximos do limite. Entretanto, pode-se observar que as tensões resultantes estão bem abaixo dos limites de tensão (50 Mpa e 300 Mpa para as camadas piezelétrica e de alumínio, respectivamente), mostrando que não é necessária uma restrição de tensão neste problema. Os valores máximos de tensão de von Mises, seguindo a sequência de empilhamento piezelétrico/alumínio/piezelétrico são iguais a 17,08, 25,53 e 13,63 MPa.

As Figs. 4.10, 4.11 e 4.12(a) referem-se aos resultados de otimização de topologia, de polarização e de orientação para o caso 2 e a Fig. 4.12(b) apresenta sua respectiva deformada. O valor de deslocamento obtido neste caso é de $29 \mu \mathrm{m}, 29,7 \%$ maior que no caso 1 e $74 \%$ maior que no caso 0. Pela Fig. 4.10, é possível notar que os volumes finais das camadas interna e externa são diferentes que no caso 1 e são iguais a 89, 37\% e 90, 87\% , respectivamente. O aumento nos deslocamentos do ponto $A$ no caso 2 foi possível por causa da otimização da orientação das fibras da camada de fibra de carbono, já que o resultado de otimização de polarização é bem parecido com o do caso 1 .

Como pode ser observado nos resultados de otimização de topologia das Figs. 4.6 e 4.10, foram obtidas configurações quase 0-1 para a distribuição de material, o que, para a aplicação proposta, confirma que a técnica de projeção com a função "degrau" é eficiente em eliminar pseudo-densidades intermediárias. Para os resultados de polarização das Figs. 4.7 e 4.11, pode ser observado algumas regiões com materiais intermediários, representando polarização intermediária. Entretanto, essas regiões coincidem com 


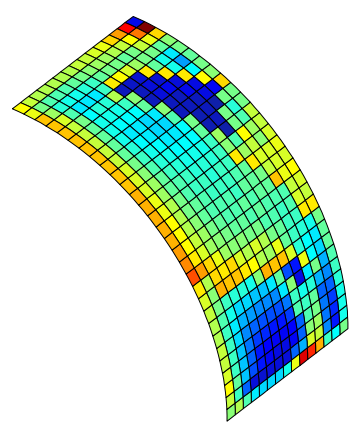

(a) Camada piezelétrica interna - Superfície inferior

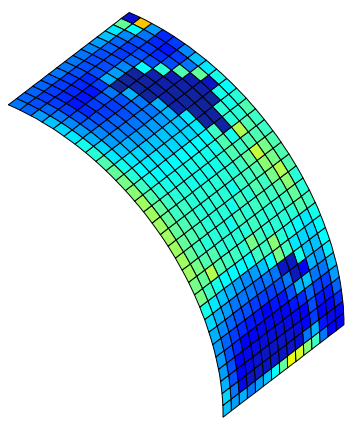

(b) piezelétrica - Superfície superior

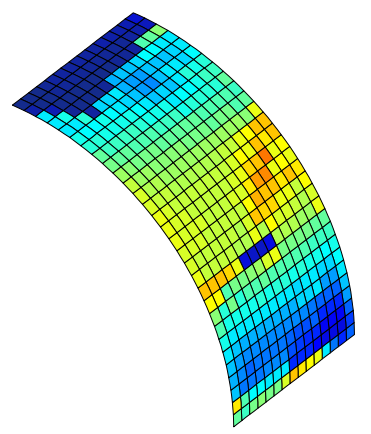

(c) piezelétrica externa - Superfície inferior

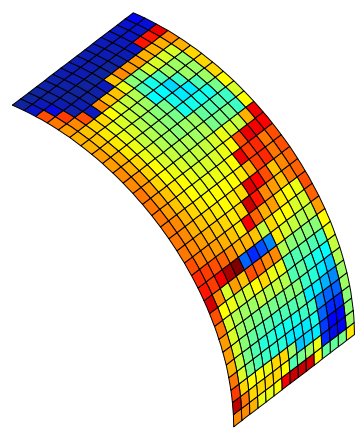

(d) Camada piezelétrica externa - Superfície superior

Figura 4.9: Distribuição das tensões de von Mises das camadas de material piezelétrico para o caso 1 de atuadores de casca. Valores de tensão em $\mathrm{MPa}$, onde o valor máximo da escala representa o valor máximo de tensão nas camadas piezelétricas.

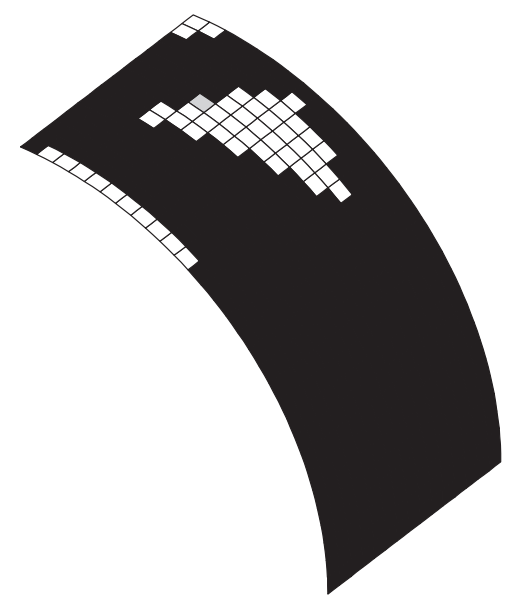

(a) Camada piezelétrica interna

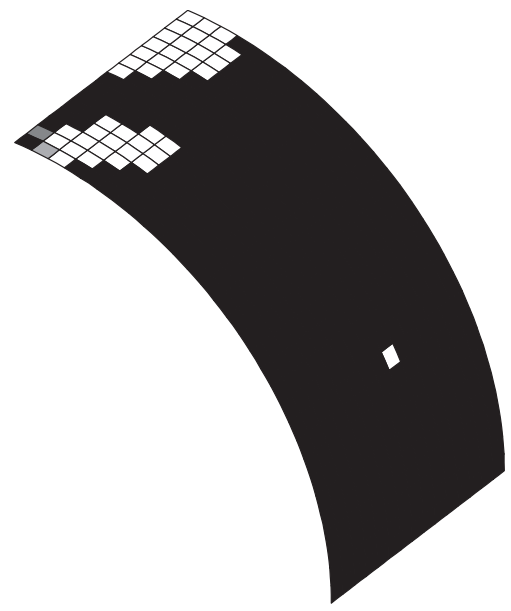

(b) Camada piezelétrica externa

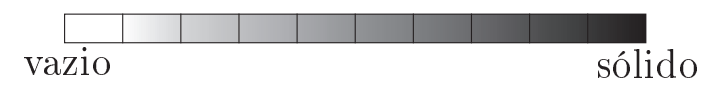

Figura 4.10: Resultado da otimização de topologia para o caso 2 de atuadores de casca.

áreas sem material dos resultados de otimização topológica, e assim essas regiões com polarização intermediária podem ser desconsideradas.

Na Fig. 4.13 são apresentadas as distribuições de tensão de von Mises nas superfícies inferior e superior apenas das camadas de material piezelétrico, por apresentarem valores 


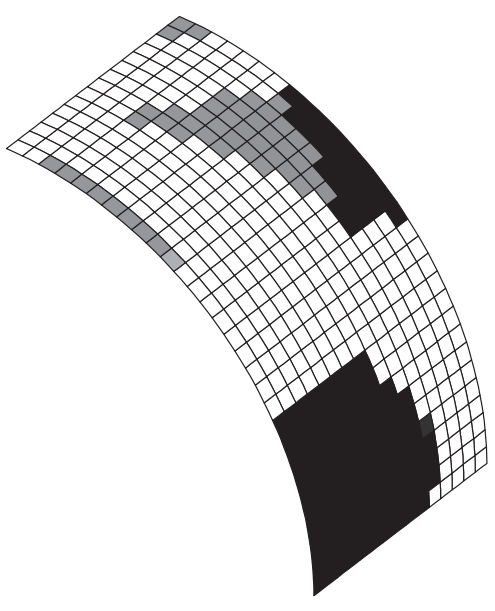

(a) Camada piezelétrica interna

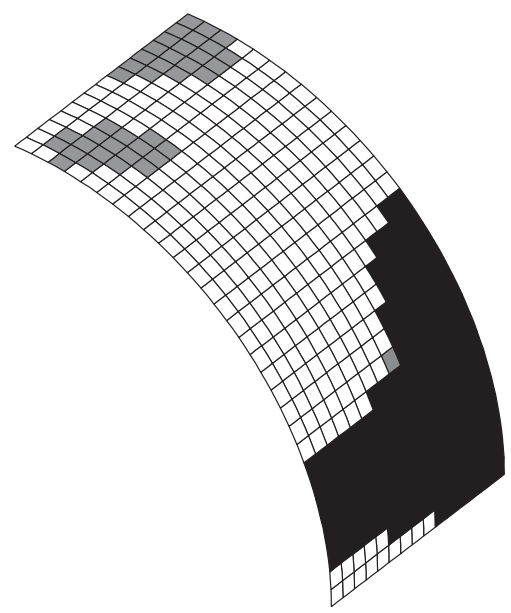

(b) Camada piezelétrica externa

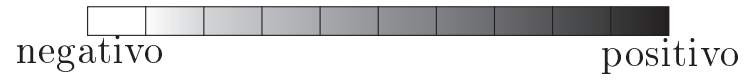

Figura 4.11: Resultado da otimização de polarização para o caso 2 de atuadores de casca.

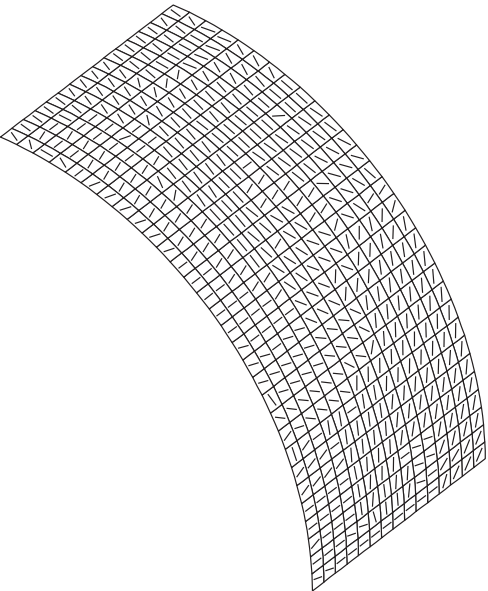

(a) Camada de fibra de carbono

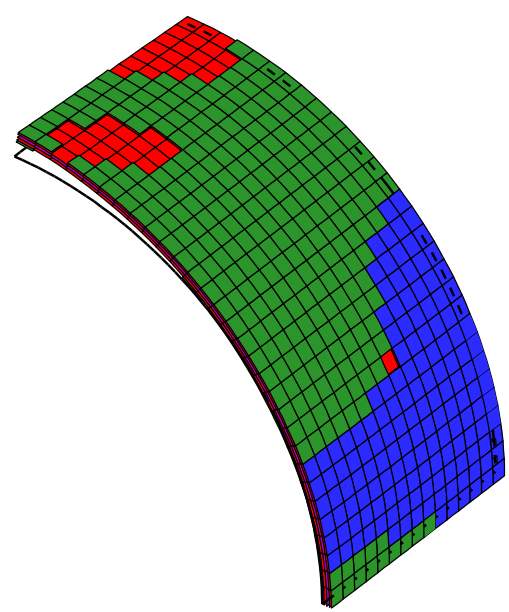

(b) Forma deformada

Figura 4.12: Resultado da otimização de orientação de fibras e a forma deformada da estrutura para o caso 2 de atuadores de casca.

mais próximos do limite do que as outras camadas. Entretanto, pode-se observar que as tensões resultantes estão bem abaixo dos limites de tensão para cada tipo de material (50 Mpa e 300 Mpa para as camadas piezelétrica e de alumínio, respectivamente). Os valores de tensão de von Mises das camadas interna e externa de material piezelétrico são iguais a 15,21 e 13,90 MPa, respectivamente. Para as camadas de alumínio são iguais a 33,68 e 21,74 MPa. Na camada de fibra de carbono, o valor máximo da tensão de Tsai-Wu é igual 


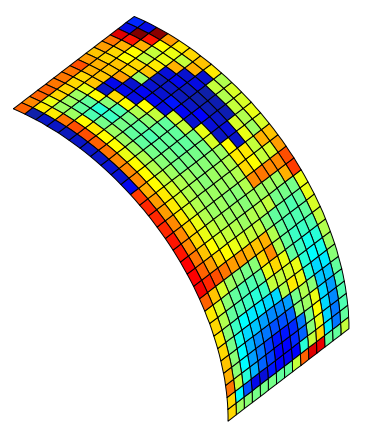

(a) Camada piezelétrica interna - Superfície inferior

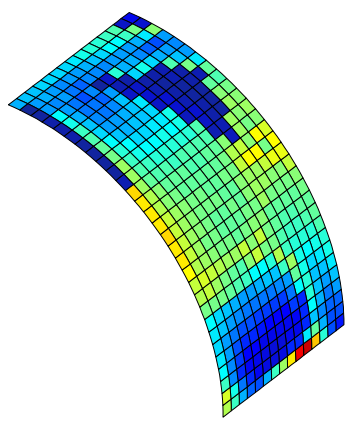

(b) Camada piezelétrica interna - Superfície superior

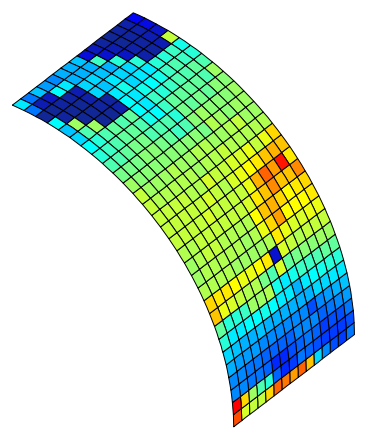

(c) Camada piezelétrica externa - Superfície inferior

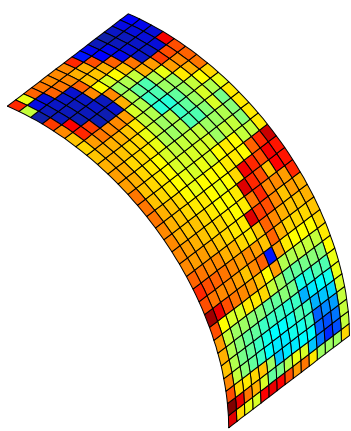

(d) Camada piezelétrica externa - Superfície superior

Figura 4.13: Distribuição das tensões de von Mises das camadas de material piezelétrico para o caso 2 de atuadores de casca. Valores de tensão em $\mathrm{MPa}$, onde o valor máximo da escala representa o valor máximo de tensão nas camadas piezelétricas.

a 0,05, ou seja, muito abaixo do limite de falha, que é igual a 1. Dado que as tensões nas camadas de alumínio e de fibra de carbono são menores do que $10 \%$ do limite de tensão, não são apresentadas as distribuições de tensão para essas camadas. Assim, é possível afirmar que a restrição de tensão não é necessária nesse exemplo.

A orientação das fibras tendem a deixar o substrato de fibra de carbono mais flexível para o movimento solicitado. Entretanto, as técnicas de fabricação de compósitos que existem atualmente não garantem uma discretização tão pequena das fibras, ou seja, não é possível atribuir um ângulo para cada elemento. Assim, deve-se utilizar algumas restrições na otimização de fibras. Neste trabalho, propõe-se atribuir as variáveis de orientação a grupos de elementos adjacentes de modo que o mesmo ângulo de fibra cubra uma área maior.

Assim, é apresentado um outro resultado considerando essa restrição de fibras, sendo que os elementos da camada de fibra de carbono são agrupados em duas grandes regiões para que se tenha apenas dois ângulos na camada. Isso facilita a manufatura desses transdutores, sendo possível fabricar até mesmo pelo método manual de infusão a vácuo de resina. As Figs. 4.14, 4.15 e 4.16(a) referem-se aos resultados de otimização de topologia, de polarização e de orientação para este caso de orientações agrupadas (caso 3) e a Fig. 4.16(b) apresenta sua respectiva deformada.

O valor de deslocamento obtido nesse caso é de $27,07 \mu \mathrm{m}$, que é $21 \%$ maior do que no 


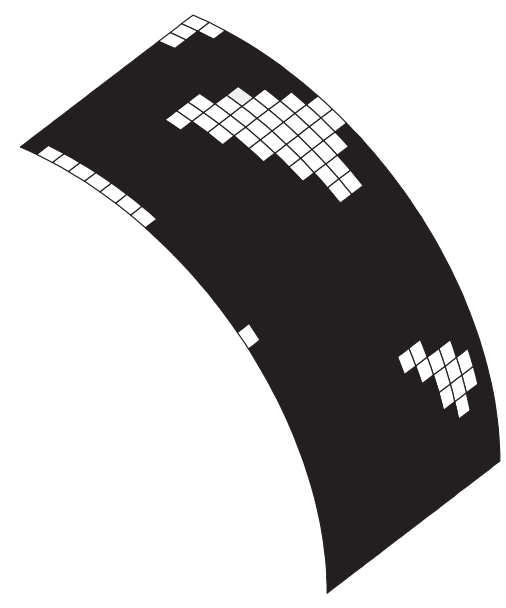

(a) Camada piezelétrica interna

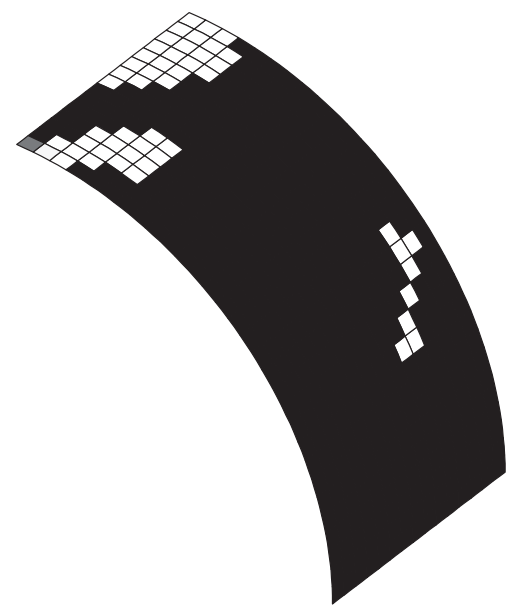

(b) Camada piezelétrica externa

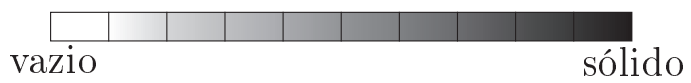

Figura 4.14: Resultado da otimização de topologia para o caso 3 de atuadores de casca.

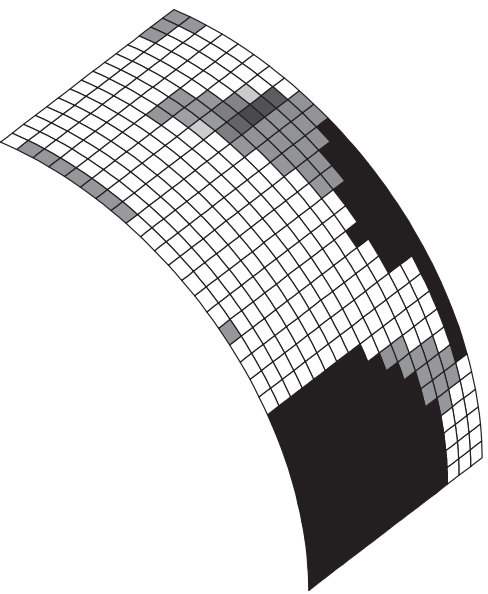

(a) Camada piezelétrica interna

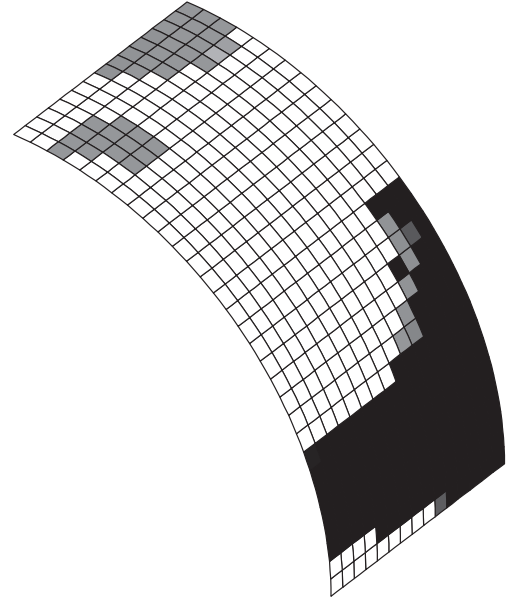

(b) Camada piezelétrica externa

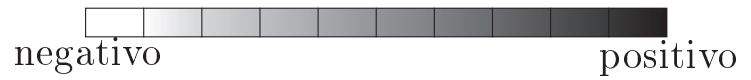

Figura 4.15: Resultado da otimização de polarização para o caso 3 de atuadores de casca.

caso 1, mas é 7,13\% menor do que no caso 2. Essa redução no valor do deslocamento no caso 3 comparado com o caso 2 já era esperado, já que não é permitido tanta liberdade para a otimização de fibras, restringindo assim, a flexibilidade do substrato. Entretanto o caso 3 se apresenta como uma solução mais viável do ponto de vista de fabricação, já que apresenta uma melhora no desempenho quando comparado com o caso 1. 


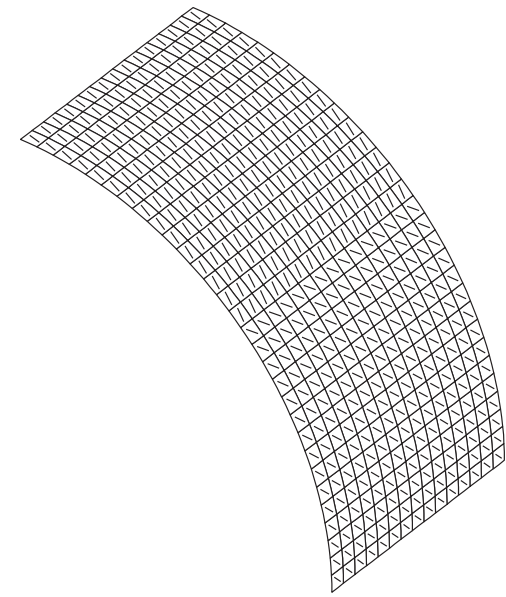

(a) Camada de fibra de carbono

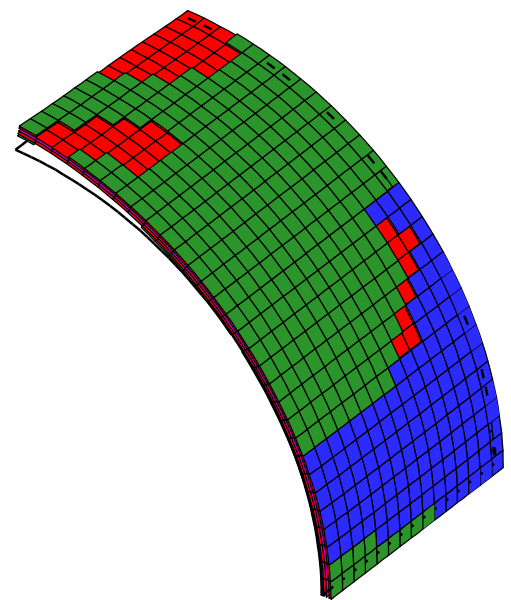

(b) Forma deformada

Figura 4.16: Resultado da otimização de orientação de fibras e a forma deformada da estrutura para o caso 3 de atuadores de casca.

Na Fig. 4.17 são apresentadas as distribuições de tensão de von Mises nas superfícies inferior e superior apenas das camadas de material piezelétrico, por apresentarem valores mais próximos do limite do que as outras camadas. Entretanto, pode-se observar que as tensões resultantes estão bem abaixo dos limites de tensão para cada tipo de material. Os valores de tensão de von Mises das camadas de material piezelétrico e alumínio são iguais a 15,25, 34,28, 20,86 e 14,61 MPa, seguindo a sequência de empilhamento piezelétrico/alumínio/alumínio/piezelétrico, sendo que as tensões admissíveis são iguais a 50 e $300 \mathrm{MPa}$, para as camadas piezelétricas e de alumínio. Na camada de fibra de carbono o valor máximo da tensão de Tsai-Wu é igual a 0,05 , ou seja, muito abaixo do limite de falha, que é igual a 1. Assim, é possível afirmar que a restrição de tensão também não é necessária neste exemplo.

A Tab. 4.1 apresenta os valores de deslocamento vertical no ponto $A\left(u_{y_{a}}\right)$, de tensão máxima de von Mises nas camadas piezelétricas $\left(\sigma_{v m_{\text {max }}}^{\text {inf }}\right.$ e $\left.\sigma_{v m_{\text {max }}}^{\text {sup }}\right)$ e de volume final das duas camadas piezelétricas otimizadas $\left(V_{o t}^{\text {inf }}\right.$ e $V_{o t}^{\text {sup }}$ ) para todos os casos apresentados.

Tabela 4.1: Dados dos resultados apresentados para o exemplo de atuadores de casca.

\begin{tabular}{lccccc}
\hline & $\begin{array}{c}u_{y_{A}} \\
\mu \mathrm{m}\end{array}$ & $\begin{array}{c}\sigma_{v m_{\max }}^{\text {inf }} \\
\mathrm{MPa}\end{array}$ & $\begin{array}{c}\sigma_{v m_{\max }}^{\text {sup }} \\
\mathrm{MPa}\end{array}$ & $\begin{array}{c}V_{\text {ot }}^{\text {inf }} \\
\%\end{array}$ & $\begin{array}{c}V_{\text {ot }}^{\text {sup }} \\
\%\end{array}$ \\
\hline caso 0 & 16,67 & - & - & - & - \\
caso 1 & 22,36 & 17,08 & 13,63 & 93,28 & 88,57 \\
caso 2 & 29,00 & 15,21 & 13,90 & 89,37 & 90,87 \\
caso 3 & 27,07 & 15,25 & 14,61 & 82,15 & 85,89 \\
\hline
\end{tabular}




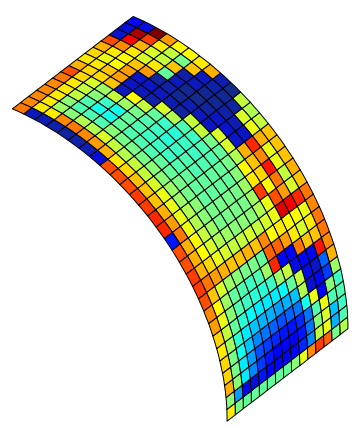

(a) Camada piezelétrica interna - Superfície inferior

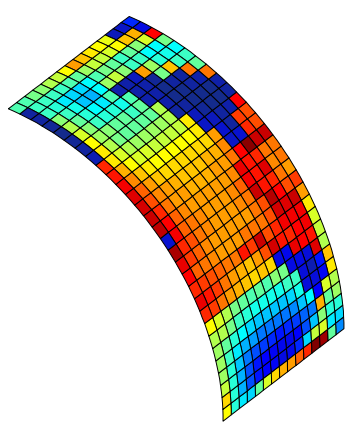

(b) Camada piezelétrica interna - Superfície superior

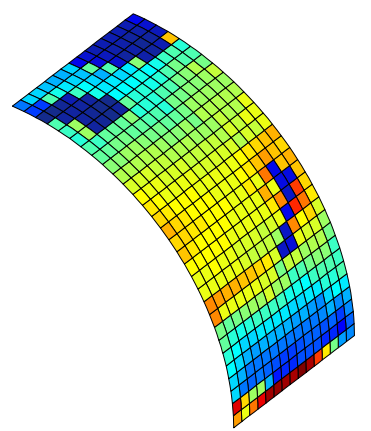

(c) Camada piezelétrica externa - Superfície inferior

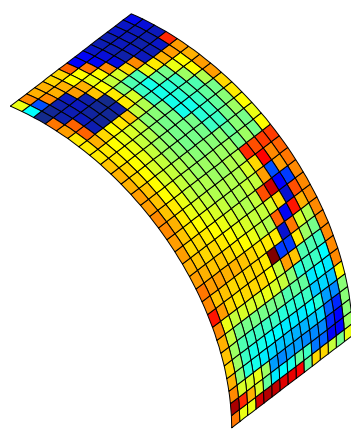

(d) Camada piezelétrica externa - Superfície superior

Figura 4.17: Distribuição das tensões de von Mises das camadas de material piezelétrico para o caso 3 de atuadores de casca. Valores de tensão em $\mathrm{MPa}$, onde o valor máximo da escala representa o valor máximo de tensão na camada piezelétrica.

\subsubsection{Exemplo 2}

Neste exemplo, é utilizado um domínio de placa quadrada, conforme apresentado na Fig. 4.18, de lados iguais a $l=300 \mathrm{~mm}$. O raio utilizado na técnica de projeção é igual a $2 \mathrm{~mm}$. Os ângulos das orientações das fibras são também representadas na Fig. 4.18 onde o ângulo $0^{\circ}$ segue o eixo $x$ e $90^{\circ}$ segue o eixo $z$. O sentido de polarização do material piezelétrico segue o sentido da direção $y$.

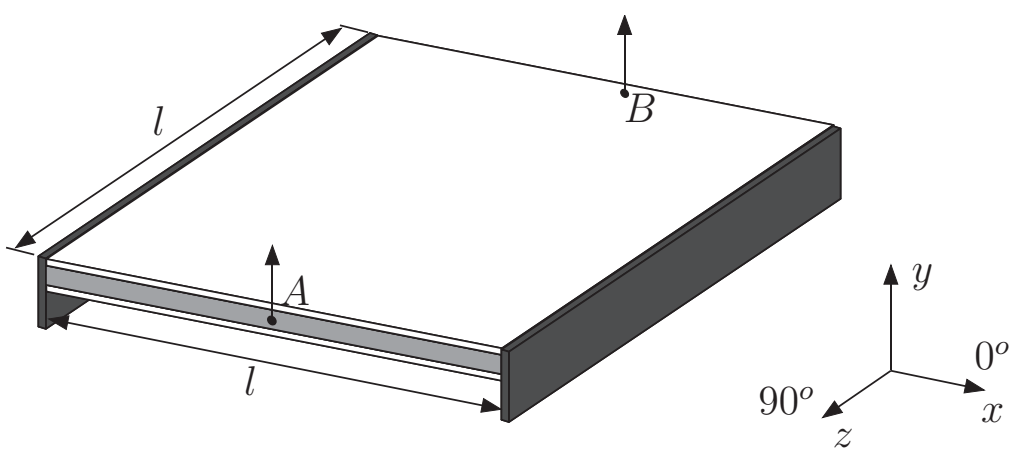

Figura 4.18: Domínio de projeto para atuadores. Medidas em mm.

A estrutura está engastada nos dois lados em contato com os blocos cinza escuro. Entretanto, somente a camada de substrato está engastada, ou seja, a camada de material piezelétrico está apenas colada sobre o substrato e não é presa no engaste. Essa abordagem aproxima a modelagem de uma estrutura real, já que a fixação geralmente é feita apenas 
no substrato.

Uma análise de convergência da malha de elementos finitos revelou que a convergência nas análises é obtida quando a estrutura é dividida em 30x30 elementos. Não foi utilizada uma malha mais refinada pois o elemento de casca utilizado, junto com o método OMD são muito custosos computacionalmente.

A Fig. 4.19 apresenta a forma deformada da estrutura sem aplicar nenhuma otimização, ou seja, as camadas de material piezelétrico são totalmente cheias e com polarização negativa para gerar deslocamentos positivos em $y$ nos pontos $A$ e $B$. Esse caso será chamado de caso 0, e obteve-se um deslocamento de 1,27 $\mathrm{mm}$ em ambos os pontos.

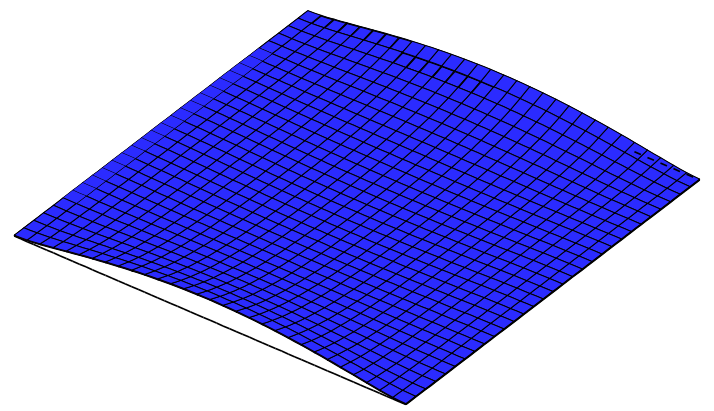

Figura 4.19: Forma deformada da estrutura para o caso 0 de atuador de placa, ou seja, sem otimização, com as camadas piezelétricas totalmente cheias e com polarização negativa.

As Figs. 4.20 e 4.21 referem-se aos resultados de otimização de topologia e de polarização para o caso 1 e a Fig. 4.22 apresenta sua respectiva deformada. Os valores de deslocamentos obtidos nos pontos $A$ e $B$ são idênticos e iguais a 2,96 mm, 133,07\% maior que no caso 0. Nota-se pela Fig. 4.20 que não é necessário que as camadas piezelétricas estejam totalmente cheias sendo que os volumes ótimos de material piezelétrico para as duas camadas são iguais a $74 \%$ do volume total da camada.

Na Fig. 4.23 são apresentadas as distribuições de tensão mecânica nas superfícies inferior e superior somente das camadas de material piezelétrico, pois são as camadas que apresentam valores de tensão mais próximos do limite. Entretanto, as tensões resultantes estão bem abaixo dos limites de tensão (que são iguais a 50 e $300 \mathrm{MPa}$ para o material piezelétrico e para o alumínio, respectivamente), mostrando que não é necessária uma restrição de tensão neste problema. Os valores máximos de tensão de von Mises, seguindo a sequência de empilhamento piezelétrico/alumínio/piezelétrico são iguais a 21,93, 19,92 e 21,93 MPa.

As Figs. 4.24, 4.25 e 4.26(a) referem-se aos resultados de otimização de topologia, 


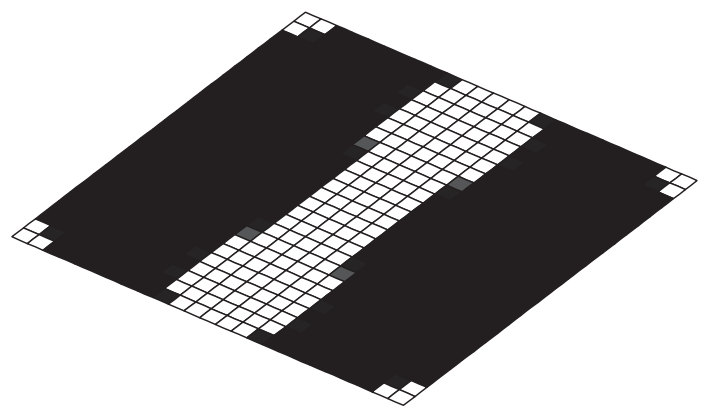

(a) Camada piezelétrica interna

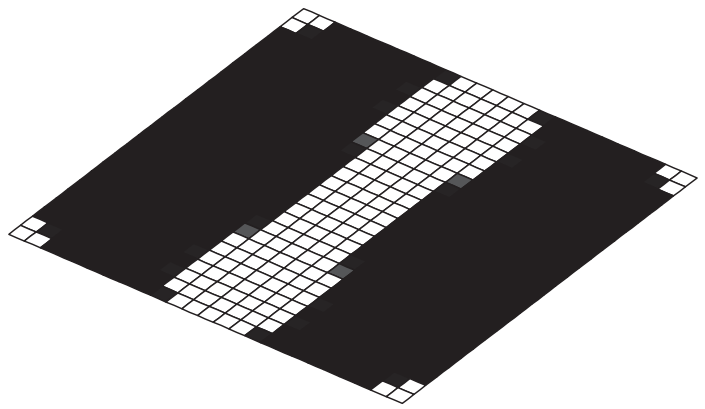

(b) Camada piezelétrica externa

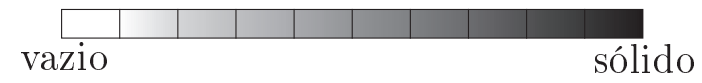

Figura 4.20: Resultado da otimização de topologia das camadas piezelétricas para o caso 1 de atuadores de placa.

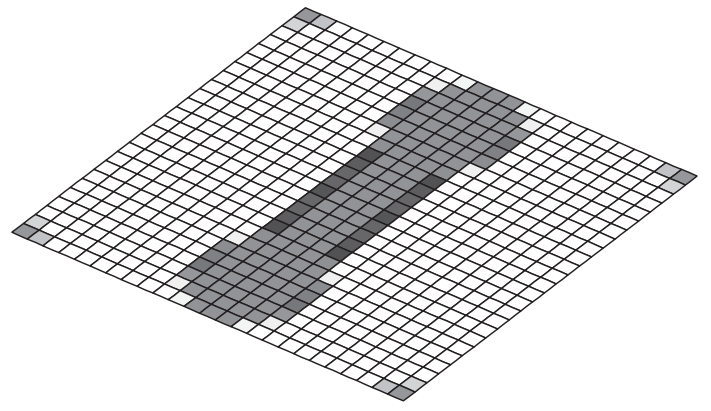

(a) Camada piezelétrica inferior

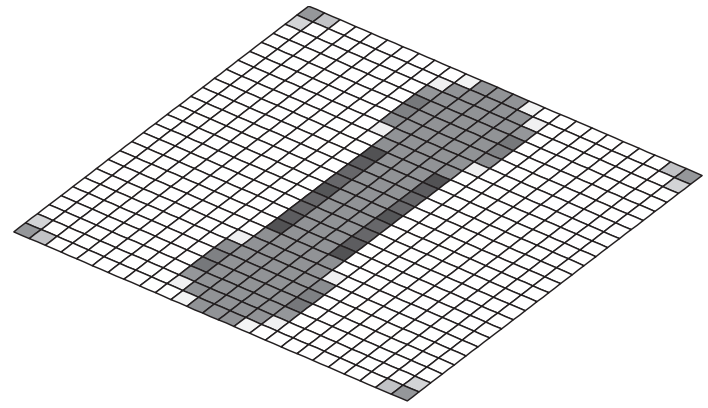

(b) Camada piezelétrica superior

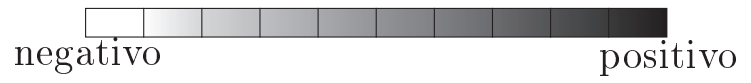

Figura 4.21: Resultado da otimização de polarização das camadas piezelétricas para o caso 1 de atuadores de placa.

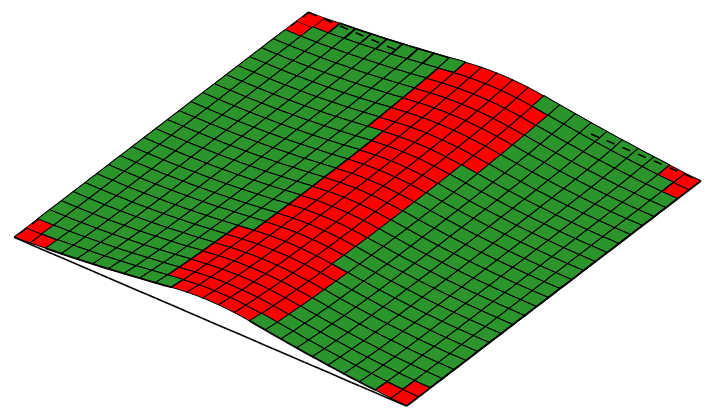

Figura 4.22: Forma deformada da estrutura para o caso 1 de atuadores de placa. Em vermelho está representada a camada de alumínio, e em verde está representada a camada piezelétrica com polarização negativa. 

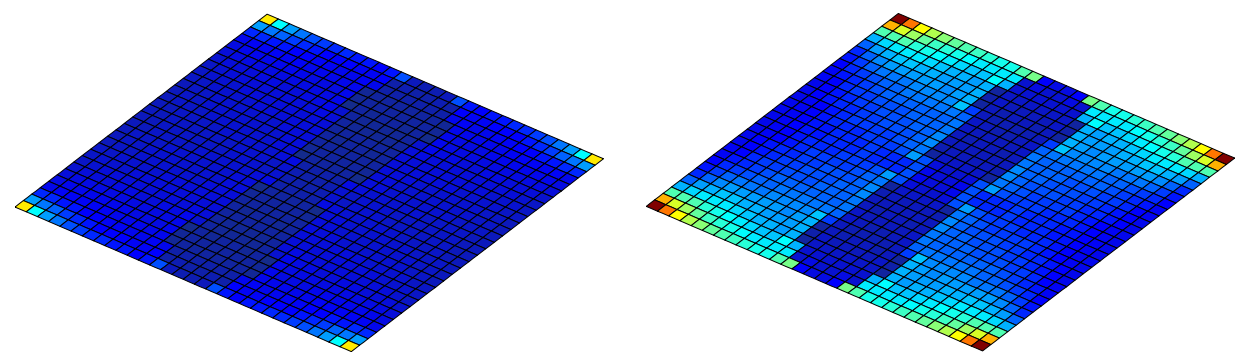

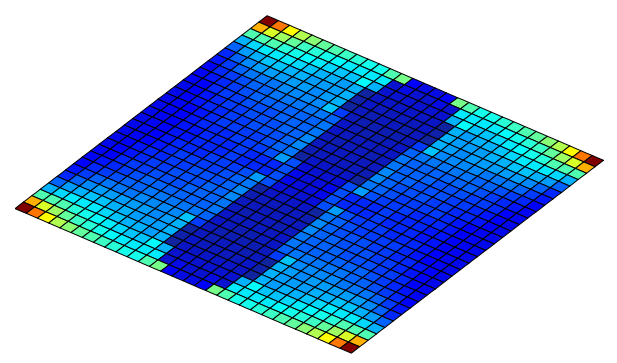

(a) Camada piezelétrica inferior

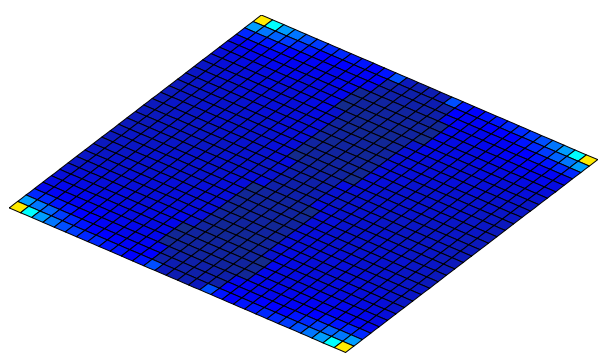

(b) Camada piezelétrica superior

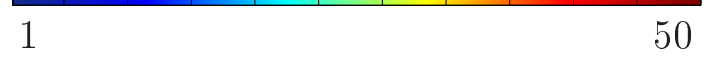

Figura 4.23: Distribuição das tensões de von Mises das superfícies superior e inferior das camadas de material piezelétrico para o caso 1 de atuadores de placa. Valores de tensão em MPa, onde os valores máximos da escala representam os limites de tensão.

de polarização e de orientação para o caso 2 e a Fig. 4.26(b) apresenta sua respectiva deformada. O valor de deslocamento obtido neste caso é de 3,21 mm, 8,44\% maior que no caso 1 e 152,75\% maior que no caso 0 . Os volumes finais para as duas camadas são iguais a $74,67 \%$ do volume total da camada. O pequeno aumento nos deslocamentos dos pontos $A$ e $B$ no caso 2, comparado como caso 1, foi possível por causa da otimização da orientação das fibras da camada de fibra de carbono, já que os resultados de otimização de topologia e de polarização são semelhantes aos resultados do caso 1.

Nos resultados de otimização de topologia das Figs. 4.20 e 4.24, foram obtidas configurações quase 0-1 para a distribuição de material, o que, para a aplicação proposta, confirma que a técnica de projeção com a função "degrau" é eficiente em eliminar pseudodensidades intermediárias. Já para os resultados de polarização das Figs. 4.21 e 4.25 pode ser observado algumas regiões com escala de cinza, representando polarização intermediária. Entretanto, essas regiões coincidem com áreas sem material dos resultados de otimização topológica, e assim essas regiões com polarização intermediária podem ser desconsideradas.

Na Fig. 4.27 são apresentadas as distribuições de tensão de von Mises nas superfícies inferior e superior apenas das camadas de material piezelétrico. A distribuição de tensão 


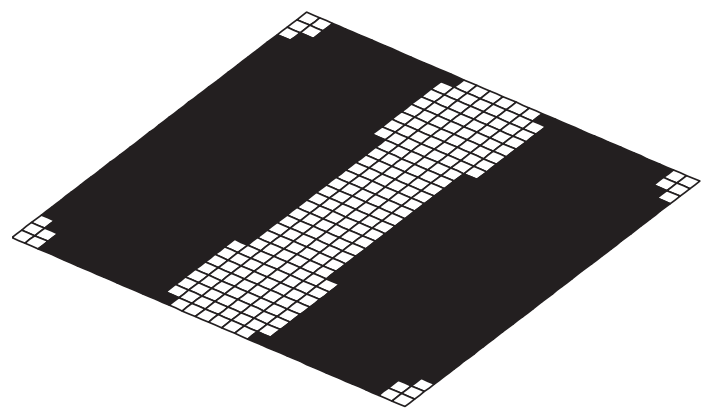

(a) Camada piezelétrica interna

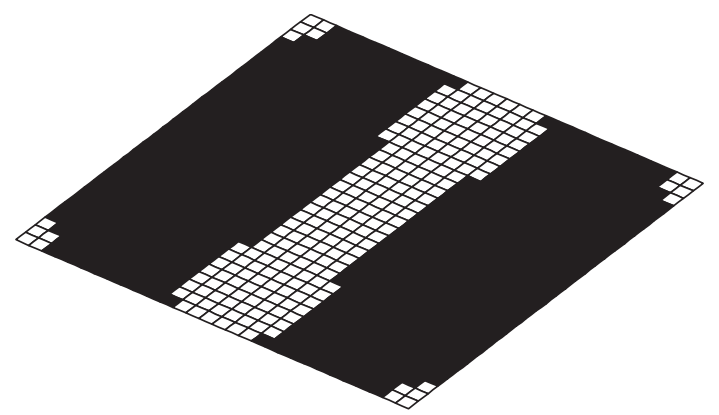

(b) Camada piezelétrica externa

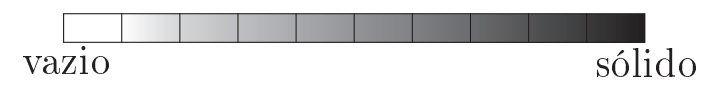

Figura 4.24: Resultado da otimização de topologia para o caso 2 de atuadores de placa.

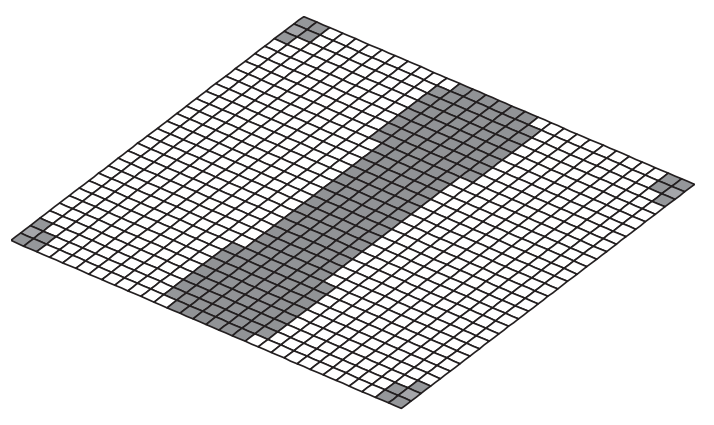

(a) Camada piezelétrica inferior

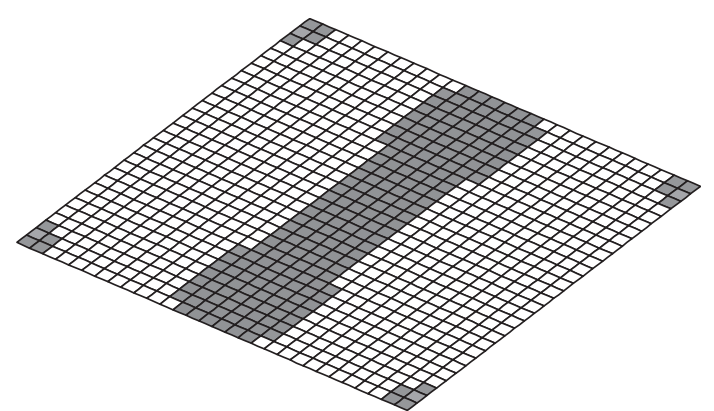

(b) Camada piezelétrica superior

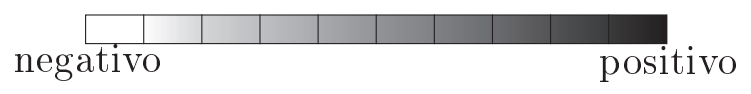

Figura 4.25: Resultado da otimização de polarização para o caso 2 de atuadores de placa.

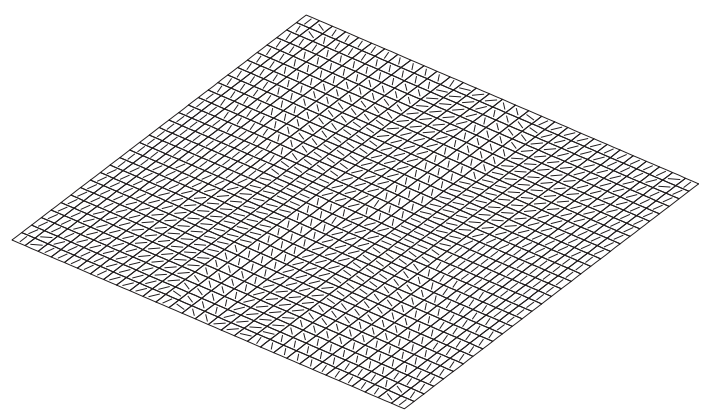

(a) Camada de fibra de carbono

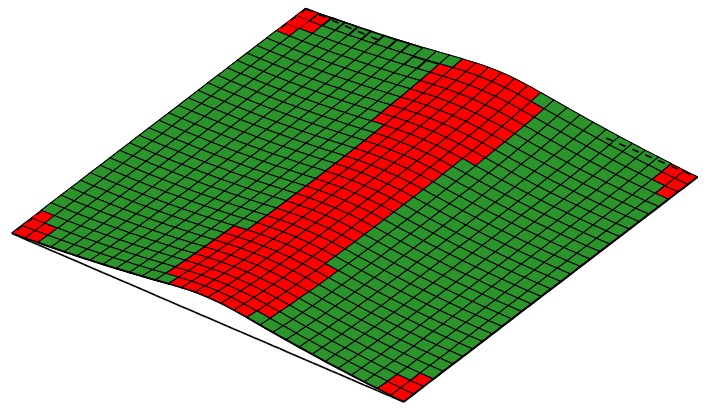

(b) Forma deformada

Figura 4.26: Resultado da otimização de orientação de fibras e a forma deformada da estrutura para o caso 2 de atuadores de placa. 


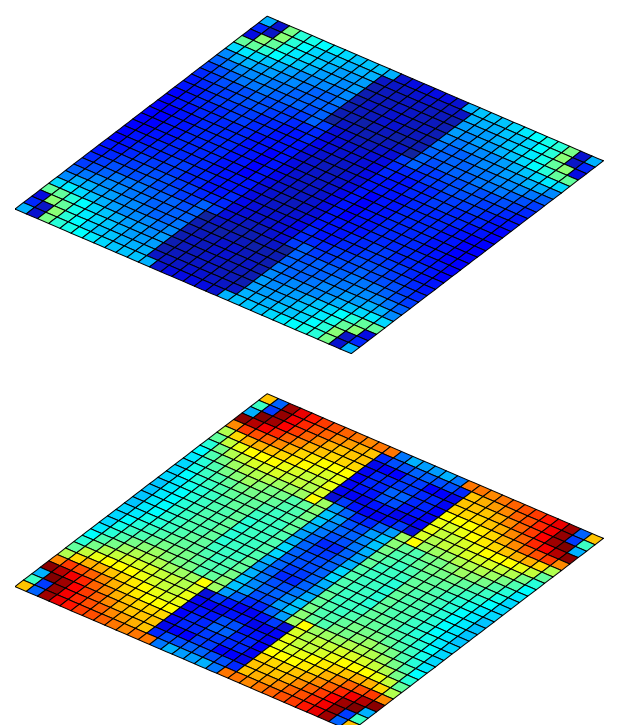

(a) Camada piezelétrica inferior

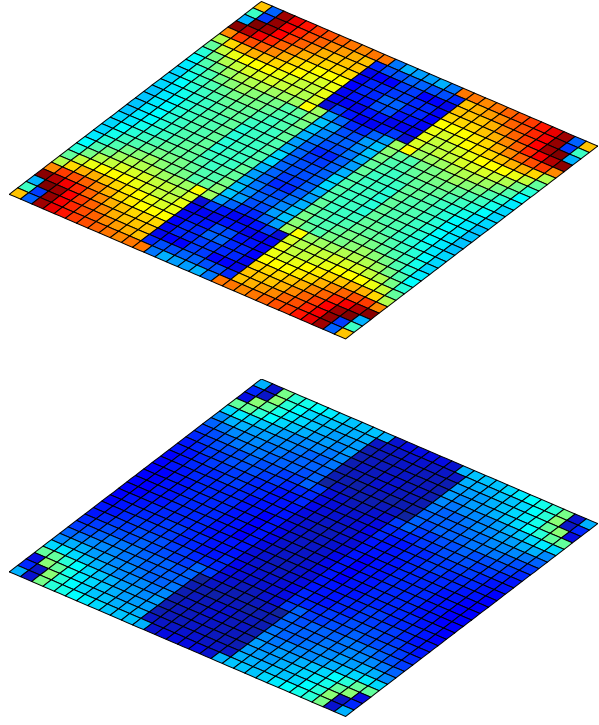

(b) Camada piezelétrica superior

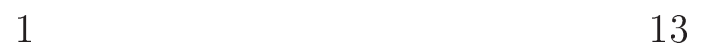

Figura 4.27: Distribuição das tensões de von Mises das camadas de material piezelétrico para o caso 2 de atuadores de placa. Valores de tensão em $\mathrm{MPa}$, onde o valor máximo da escala representam o valor máximo de tensão nas camadas piezelétricas.

das outras camadas não são apresentados por apresentarem valores menores do que 11\% do limite de tensão de cada material. Entretanto, mesmo na camada de material piezelétrico, pode-se observar que as tensões resultantes estão bem abaixo dos limites de despolarização. Os valores de tensão de von Mises das camadas de material piezelétrico são iguais a 12,85 e 12,85 MPa (tensão limite igual a $50 \mathrm{MPa}$ ), e para as camadas de alumínio são iguais a 35,64 e 35,64 MPa (tensão limite igual a $300 \mathrm{MPa}$ ). Na camada de fibra de carbono o valor máximo da tensão de Tsai-Wu é igual a 0,064, ou seja, muito abaixo do limite de falha (que é igual a 1). Assim, é possível afirmar que a restrição de tensão não é necessária nesse exemplo.

A orientação das fibras tendem a deixar o substrato de fibra de carbono mais flexível para o movimento solicitado. Entretanto, as técnicas de fabricação de compósitos que existem atualmente não garantem uma discretização tão pequena das fibras, ou seja, não é possível atribuir um ângulo para cada elemento. Assim, deve-se utilizar algumas restrições na otimização de fibras. Neste trabalho, propõe-se atribuir as variáveis de orientação a grupos de elementos adjacentes de modo que o mesmo ângulo de fibra cubra uma área maior.

Assim, é apresentado um outro resultado considerando essa restrição de fibras, sendo 
que os elementos da camada de fibra de carbono são agrupados em duas grandes regiões para que se tenha apenas dois ângulos na camada. Isso facilita a manufatura desses transdutores, sendo possível fabricar até mesmo pelo método manual de infusão a vácuo de resina. As Figs. 4.28, 4.29 e 4.30(a) referem-se aos resultados de otimização de topologia, de polarização e de orientação para este caso de orientações agrupadas (caso 3) e a Fig. 4.30(b) apresenta sua respectiva deformada.

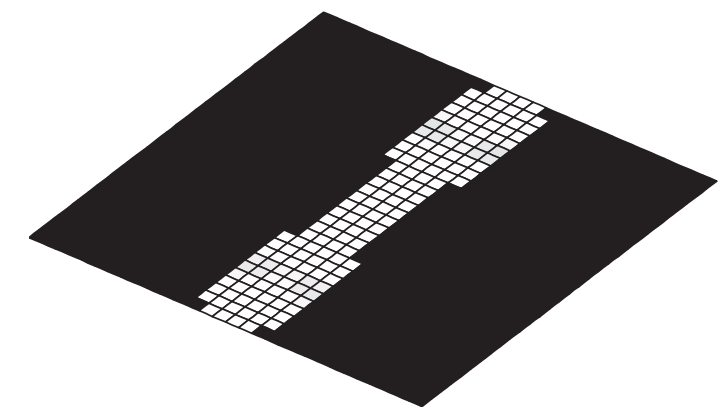

(a) Camada piezelétrica inferior

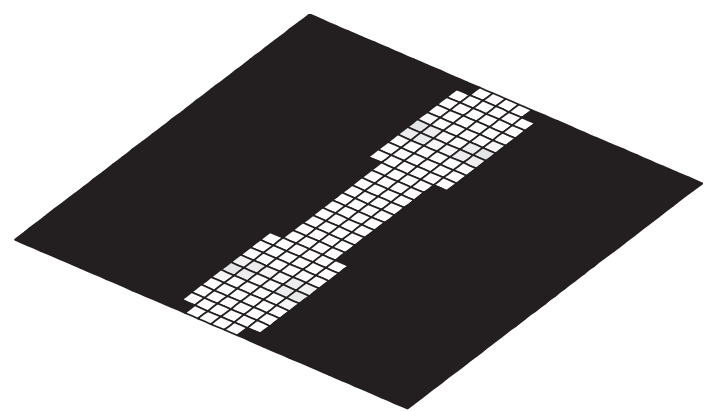

(b) Camada piezelétrica superior

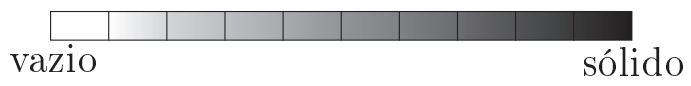

Figura 4.28: Resultado da otimização de topologia para o caso 3 de atuadores de placa

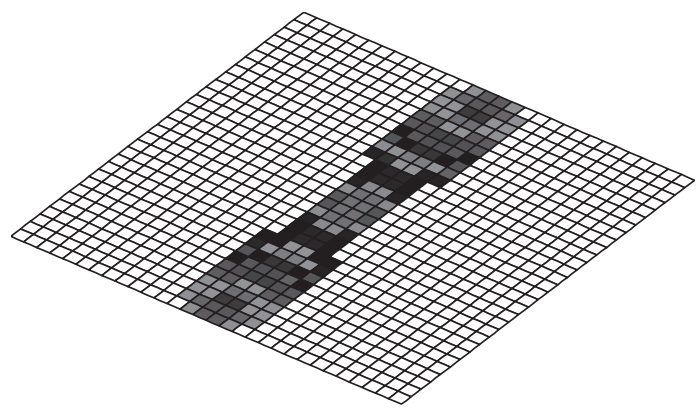

(a) Camada piezelétrica inferior

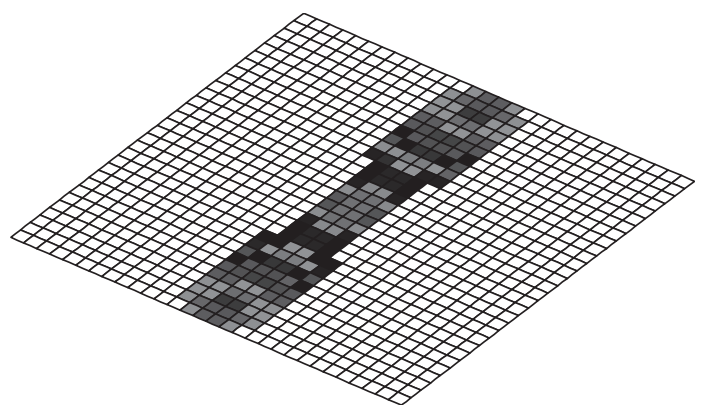

(b) Camada piezelétrica superior

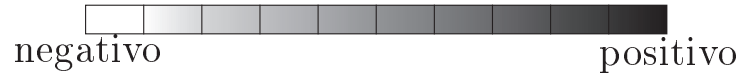

Figura 4.29: Resultado da otimização de polarização para o caso 3 de atuadores de placa.

O valor de deslocamento obtido nesse caso é de $3,19 \mathrm{~mm}$, que é 7,7\% maior do que no caso 1 , mas é $1 \%$ menor do que no caso 2 , praticamente o mesmo valor de deslocamento, já que a orientação ótima das fibras no caso 2 ficou muito semelhante ao do caso 3. Entretanto o caso 3 se apresenta como uma solução mais viável do ponto de vista de 


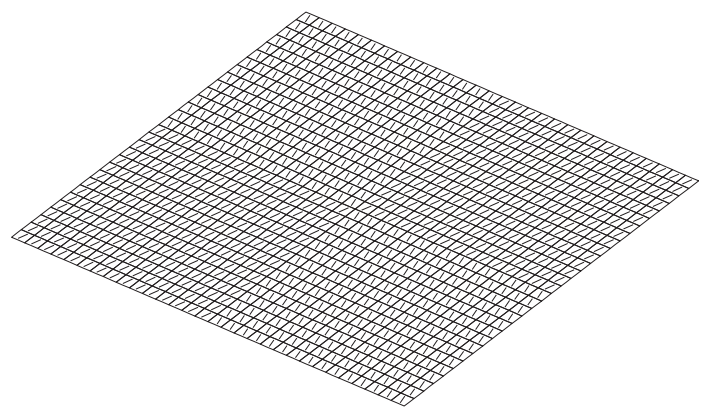

(a) Camada de fibra de carbono

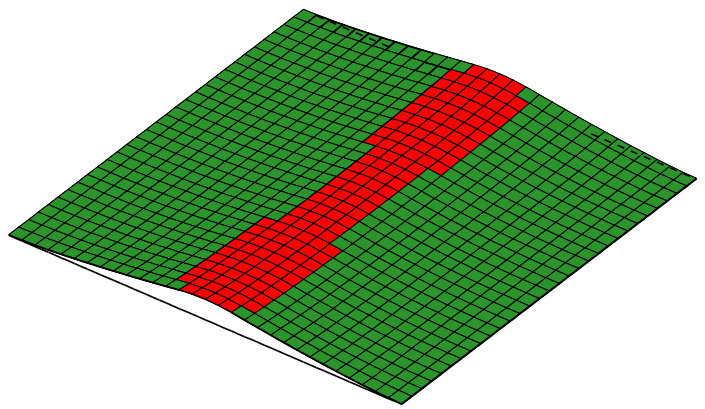

(b) Forma deformada

Figura 4.30: Resultado da otimização de orientação de fibras e a forma deformada da estrutura para o caso 3 de atuadores de placa.

fabricação, e apresenta valores bem parecidos com o caso 2. Os volumes finais para as duas camadas são iguais a 82,98\% do volume total da camada, ou seja, foi necessária uma quantidade maior de material piezelétrico para conseguir deslocamento semelhante ao do caso 2

Na Fig. 4.31 são apresentadas as distribuições de tensão de von Mises nas superfícies inferior e superior apenas das camadas de material piezelétrico. A distribuição de tensão das outras camadas não são apresentados por apresentarem valores menores do que $11 \%$ do limite de tensão de cada material. Entretanto, mesmo na camada de material piezelétrico, pode-se observar que as tensões resultantes estão bem abaixo dos limites de despolarização. Os valores de tensão de von Mises das camadas de material piezelétrico são iguais a 15,25 e 14,61 MPa (a tensão limite é igual a $50 \mathrm{MPa}$ ), e para as camadas de alumínio são iguais a 34,28 e 20,86 MPa (a tensão limite é igual a $300 \mathrm{MPa}$ ). Na camada de fibra de carbono o valor máximo da tensão de Tsai-Wu é igual a 0,05, ou seja, muito abaixo do limite de falha (que é igual a 1). Assim, é possível afirmar que a restrição de tensão também não é necessária neste exemplo.

É importante ressaltar que, neste exemplo, não foi utilizado nenhum recurso para que o resultado ficasse simétrico. A simetria obtida em todos os casos, tanto de topologia, polarização, orientação, e até de tensão mecânica são decorrentes da natureza simétrica do problema e da boa estabilidade na convergência da função objetivo.

A Tab. 4.2 apresenta os valores de deslocamento vertical nos pontos $A$ e $B\left(u_{y_{a}}\right.$ e $\left.u_{y_{b}}\right)$, de tensão máxima de von Mises nas camadas piezelétricas $\left(\sigma_{v m_{\max }}^{\text {inf }}\right.$ e $\left.\sigma_{v m_{\max }}^{\text {sup }}\right)$ e de volume final das duas camadas piezelétricas otimizadas $\left(V_{o t}^{\text {inf }}\right.$ e $\left.V_{o t}^{\text {sup }}\right)$ para todos os casos apresentados. 

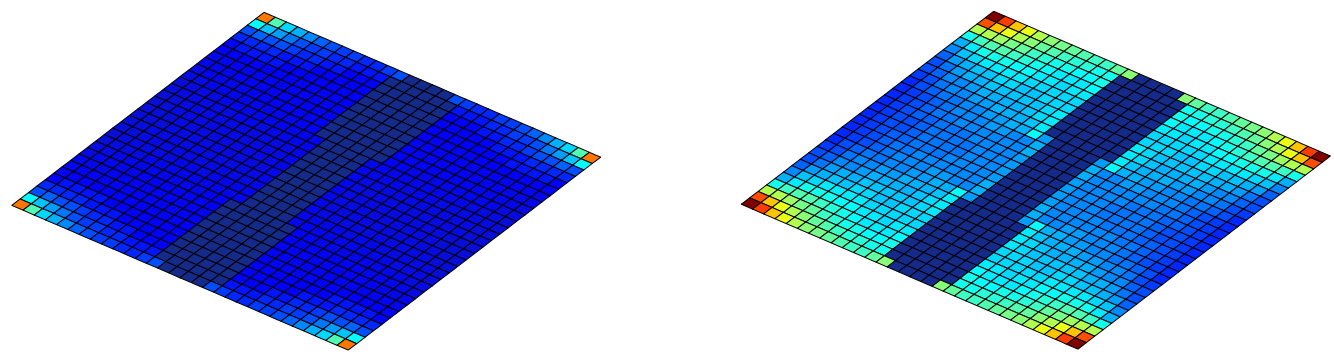

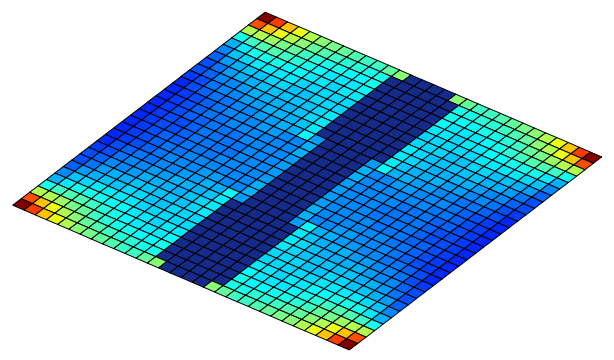

(a) Camada piezelétrica inferior

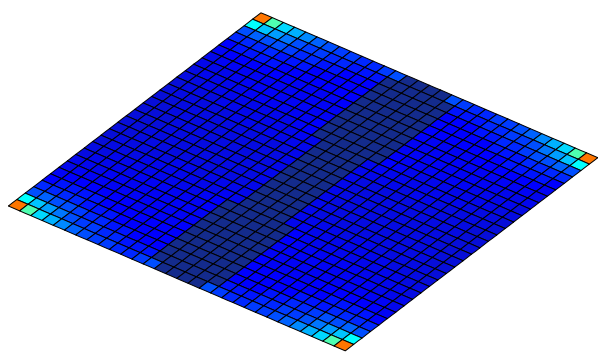

(b) Camada piezelétrica superior

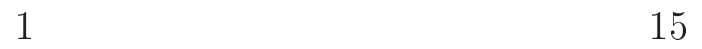

Figura 4.31: Distribuição das tensões de von Mises das camadas de material piezelétrico para o caso 3 de atuadores de placa. Valores de tensão em $\mathrm{MPa}$, onde o valor máximo da escala representa o valor máximo de tensão nas camadas piezelétricas.

Tabela 4.2: Dados dos resultados apresentados para o exemplo de atuadores de placa.

\begin{tabular}{lcccccc}
\hline & $u_{y_{A}}$ & $u_{y_{A}}$ & $\sigma_{v m_{\max }}^{\text {inf }}$ & $\begin{array}{c}\sigma_{v m_{\max }}^{\text {sup }} \\
\mathrm{mm}\end{array}$ & $\begin{array}{c}V_{o t}^{\text {inf }} \\
\%\end{array}$ & $\begin{array}{c}V_{o t}^{\text {sup }} \\
\%\end{array}$ \\
\hline caso 0 & 1,27 & 1,27 & - & - & - & - \\
caso 1 & 2,96 & 2,96 & 21,93 & 21,93 & 74 & 74 \\
caso 2 & 3,21 & 3,21 & 12,85 & 12,85 & 74,67 & 74,67 \\
caso 3 & 3,19 & 3,197 & 15,25 & 15,25 & 82,89 & 82,89 \\
\hline
\end{tabular}

\subsection{Discussão}

O projeto de atuadores piezocompósitos apresentado neste capítulo demonstra que não é necessária uma restrição de volume de material para se chegar a um resultado ótimo de topologia, já que o objetivo da otimização é maximizar o deslocamento num ponto e não reduzir o volume de material da estrutura. A análise da distribuição de tensões dos resultados otimizados também mostram que, para a ordem de grandeza de tensão elétrica (centenas de volts) que se aplica, a restrição de falha mecânica não é necessária, já que as deformações causadas pelas cerâmicas tem valor baixo.

A formulação implementada atendeu aos requisitos de projeto propostos em cada exemplo. A otimização da distribuição de material junto com a otimização de polarização 
se mostrou essencial para se obter um controle adequado do campo de deslocamentos na estrutura. Além disso, otimização da orientação das fibras se mostrou uma opção a mais para aumentar o valor do deslocamento, pois assim é possível alterar a rigidez da camada de substrato. Apesar da discretização das fibras em cada elemento não ser uma realidade atualmente, restrições podem ser impostas para que vários elementos tenham a mesma orientação. Assim, esse projeto traz um novo conceito na área de otimização topológica aplicada a transdutores piezocompósitos. 


\section{PROJETO ESTÁTICO DE SENSORES PIEZOCOMPÓSITOS DE CASCA COM RESTRIÇÃO DE TENSÃO}

Neste capítulo é apresentado o projeto otimizado de sensores piezocompósitos de casca operando em regime estático. Sensores piezocompósitos são estruturas capazes de detectar forças convertendo as deformações mecânicas em potencial elétrico. Assim, o objetivo da otimização é maximizar o potencial elétrico entre os eletrodos de cada camada piezelétrica gerado pela aplicação de uma força ou pressão, aumentando assim a sensibilidade do sensor.

Embora sensores sejam amplamente utilizados em aplicações de controle de forma (TZOU; TSENG, 1990; MUKHERJEE; JOSHI, 2002; BALAMURUGAN; NARAYANAN, 2008), apenas dois trabalhos foram encontrados abordando métodos de otimização topológica no projeto de sensores. Wang, Tai e Quek (2006) apresenta um trabalho sobre o controle da deformação por vibração torcional de placas e aplica otimização topológica nas camadas de material piezelétrico para melhorar o sensoriamento e a atuação, de modo que as deformações sejam suprimidas. Entretanto, esses autores utilizam métodos genéticos com uma malha de elementos finitos pouco refinada, e ainda assim, sua taxa de convergência é muito baixa, ou seja, são necessárias muitas iterações. Além disso, seus resultados de topologia são pouco conclusivos sem informar ao certo qual é a topologia do sensor ou do atuador. O segundo trabalho sobre o projeto de sensores utilizando o MOT foi apresentado por Nakasone (2010) em sua tese de doutorado, que aplicou o MOT para otimizar a distribuição de material piezelétrico sobre uma camada de alumínio e, além disso, otimizou o sentido de polarização desse material. Nesse sentido, o autor dessa tese submeteu recentemente um artigo (MELLO et al., Submetido em julho de 2012) com essas 
características em conjunto com outros pesquisadores do grupo.

Neste trabalho, propõe-se aplicar técnicas de otimização topológica no projeto de sensores baseados em estruturas de casca considerando não somente materiais isotrópicos e piezelétricos, mas também materiais ortotrópicos, cujas fibras são adequadamente orientadas para se maximizar o potencial elétrico no sensor. Além disso, o projeto de sensores apresentado nesta tese analisa também a distribuição de tensões mecânicas na estrutura. Esta análise é importante pois nem sempre é possível controlar as pressões aplicadas na estrutura, que podem gerar grandes deformações e, consequentemente, altos valores de tensão mecânica. Assim, a otimização deve distribuir material piezelétrico, escolher o sentido de polarização e orientar as fibras do material ortotrópico de maneira adequada para maximizar o potencial elétrico gerado pelo sensor e garantindo que os limites de tensão não sejam ultrapassados. Como já foi dito anteriormente (ver seção 3.3), esse trabalho utiliza três tipos de materiais, isotrópicos, ortotrópicos e piezelétricos e, para cada tipo de material, é utilizado um diferente limite de tensão. Neste projeto são utilizados o limite de escoamento do material isotrópico, o limite de despolarização do material piezelétrico e o limite de ruptura das fibras do material ortotrópico.

Portanto, a metodologia aqui apresentada traz importantes contribuições, não somente na área de projeto de sensores, mas também em projetos envolvendo restrição de falha mecânica.

Este capítulo está organizado da seguinte maneira: primeiramente é apresentada a modelagem dos eletrodos pelo MEF piezelétrico de casca seguida pela formulação do problema de otimização topológica para sensores, para o domínio contínuo e para o domínio discretizado. A próxima seção mostra o cálculo das sensibilidades da função objetivo e das restrições com relação às variáveis de projeto. Na sequência são apresentados resultados numéricos para consolidar a metodologia proposta. Finalmente, as conclusões desta parte do trabalho são apresentadas.

\subsection{Modelagem dos Eletrodos no MEF Piezelétrico de Casca}

No caso do projeto de sensores e coletores de energia, considera-se que os graus de liberdade de potencial elétrico nos elementos $(\Delta \phi)$ de toda uma camada estão acoplados de forma a produzirem apenas um valor de potencial elétrico, ou seja, esse acoplamento representa a utilização de eletrodos nas superfícies dessa camada. Da mesma forma que 
no acoplamento entre camadas (seção 2.7), deve-se criar uma matriz de acoplamento dos potenciais elétricos de uma mesma camada e introduzí-la no sistema de equações de equilíbrio através dos multiplicadores de lagrange.

Primeiramente, adota-se um grau de liberdade de potencial elétrico mestre, geralmente o primeiro grau de liberdade daquela camada. A matriz de acoplamento deve ser criada para que todos os outros potenciais elétricos naquela mesma camada tenham o mesmo valor do potencial elétrico do grau mestre. Assim, o sistema de acoplamento dos potenciais elétricos pode ser escrito da seguinte maneira:

$$
\begin{aligned}
& {\left[\begin{array}{cccccc}
1 & -1 & 0 & 0 & & 0 \\
1 & 0 & -1 & 0 & \cdots & 0 \\
1 & 0 & 0 & -1 & & 0 \\
& \vdots & & & \ddots & \\
1 & 0 & 0 & 0 & & -1
\end{array}\right]\left\{\begin{array}{c}
\Delta \phi_{1} \\
\Delta \phi_{2} \\
\Delta \phi_{3} \\
\vdots \\
\Delta \phi_{\text {nec }}
\end{array}\right\}=\left\{\begin{array}{c}
0 \\
0 \\
0 \\
\vdots \\
0
\end{array}\right\}} \\
& \mathbf{A}_{E} \Delta \boldsymbol{\Phi}=\mathbf{0}
\end{aligned}
$$

onde nec é o número de elementos na camada em questão e também representam os graus de liberdade de potencial elétrico de eletrodo.

No projeto de sensores apresentado nesta tese, é considerado o regime estático, assim como no projeto de atuadores. Assim, a matriz $\mathbf{A}_{E}$ deve ser introduzida no sistema de equações de equilíbrio da Eq. (4.1). Além disso, é aplicado somente carregamento de pressão na estrutura. Assim, $\mathbf{Q}=\mathbf{0}$ pois se refere às cargas elétricas internas da casca e $\mathbf{P}=\mathbf{0}$ pois considera-se que os terminais dos eletrodos estão em aberto. Portanto, o sistema de equações de equilíbrio para o caso de sensores fica:

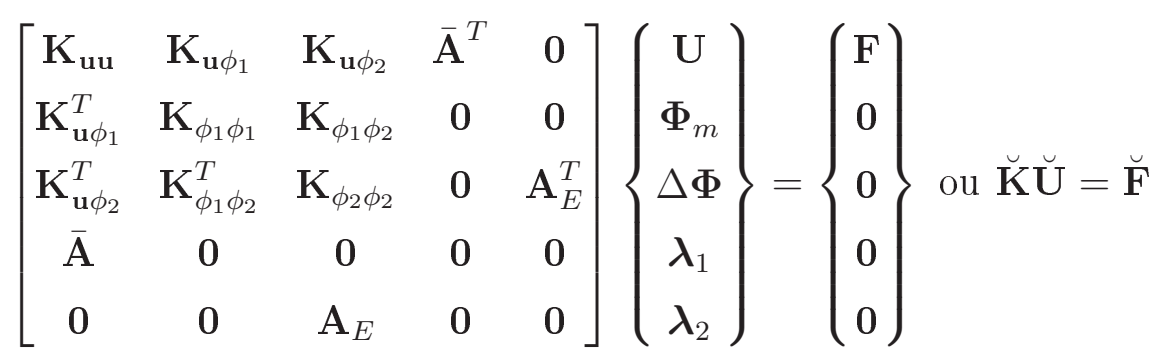

sendo que esse sistema é resolvido pelo Matlab. 


\subsection{Formulação do Problema de OT para Sensores no Domínio Contínuo}

Considere o mesmo domínio genérico apresentado na Fig. 4.1 sendo que, neste caso, $\mathbf{f}_{2}$ é uma pressão aplicada em uma determinada região $\Gamma_{f_{a}}$ que gera o potencial elétrico $\Delta \phi_{2}$ de saída na região $\Gamma_{\phi}$ e também os deslocamentos $\mathbf{U}_{2}$ em todo o domínio $\Gamma_{\Omega}$. A Fig. 5.1 ilustra esse novo conceito.

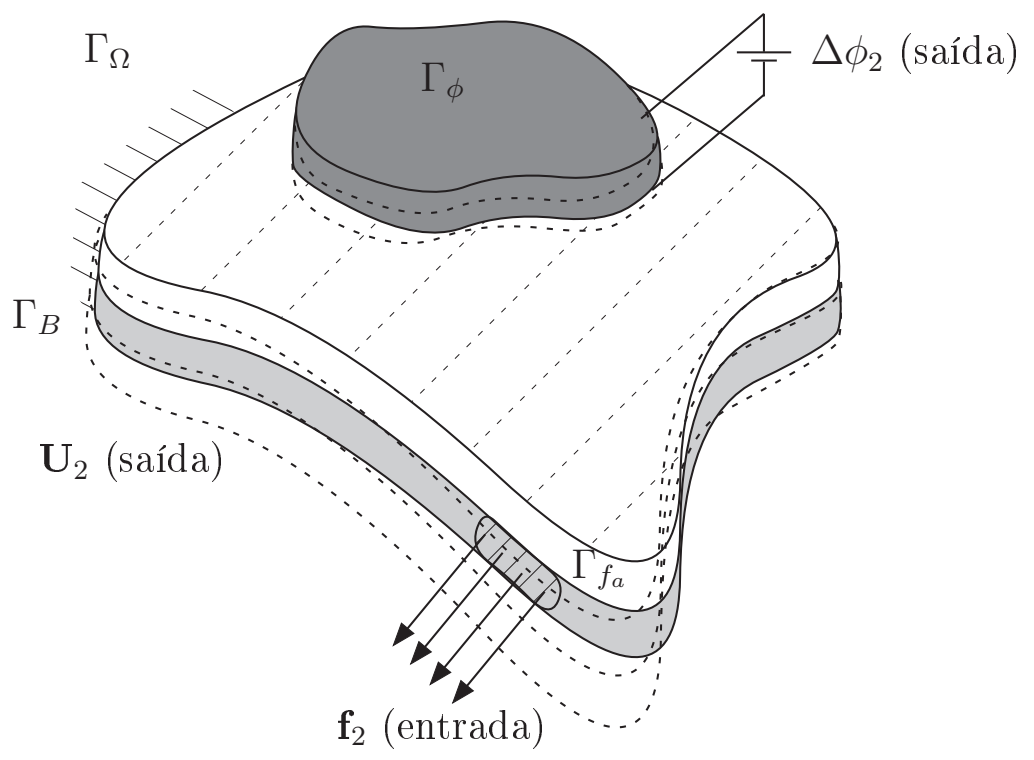

Figura 5.1: Representação do domínio de projeto de um sensor piezocompósito. A aplicação de $\mathbf{f}_{2}$ gera os deslocamentos $\mathbf{U}_{2}$ e o potencial elétrico $\Delta \phi_{2}$.

Assim, a função objetivo para maximizar o potencial elétrico, pode então, ser escrita da seguinte maneira:

$$
L_{2}=\int_{\Gamma_{\phi}} \Delta \phi_{2} d \Gamma_{\Omega}
$$

Como restrição de projeto, deseja-se garantir que o valor mínimo do fator de segurança calculado em todo o domínio seja maior ou igual a 1. Assim, utiliza-se a função "norma-p" da Eq. (3.46) para se calcular uma aproximação do valor do fator de segurança mínimo de modo que a derivada da restrição seja contínua.

Assim como no projeto de atuadores, no domínio contínuo, a distribuição de material, a polarização do material piezelétrico e a orientação das fibras do material ortotrópico são parametrizadas pelas pseudodensidades $\gamma$, pelas variáveis de polarização $\varrho$ e pelos ângulos $\boldsymbol{\theta}$, respectivamente. Portanto, o problema de otimização para sensores piezocompósitos 
pode ser formulado, no domínio contínuo, da seguinte maneira:

$$
\begin{array}{cl}
\underset{\boldsymbol{\gamma}, \boldsymbol{\varrho}, \boldsymbol{\theta}}{\operatorname{Maximizar}:} & L_{2}=\int_{\Gamma_{\phi}} \Delta \phi_{2} d \Gamma_{\Omega} \\
\text { sujeito à: } & \text { Equações de equilíbrio } \\
& s_{N P}=\left(\sum_{g=1}^{n_{\sigma}} s_{g}^{p_{\sigma}}\right)^{1 / p_{\sigma}} \geq 1 \\
& 0 \leq \boldsymbol{\gamma}(\mathbf{x}) \leq 1 \\
& 0 \leq \boldsymbol{\varrho}(\mathbf{x}) \leq 1 \\
& -90^{\circ} \leq \boldsymbol{\theta}(\mathbf{x}) \leq 90^{\circ}
\end{array}
$$

onde $\Delta \phi_{2}$ é o potencial elétrico obtido devido à aplicação da pressão $\mathbf{f}_{2}, s_{N P}$ é a "norma-p" dos fatores de segurança $s_{g}$ e $p_{\sigma}$ é o coeficiente de penalização da "norma-p" que deve ser negativo, que no caso deste trabalho $p_{\sigma}=-12$ apresenta uma boa aproximação do valor mínimo de $s_{g}$. Além disso, as equações de equilíbrio estão sujeitas às equações de acoplamento entre camadas, descrita na Seção 2.7. No projeto de sensores, também está sendo adotada uma formulação de otimização topológica sem restrição de volume, como pode ser observado na Eq. (5.5). Assim, é possível obter o volume ótimo de material piezelétrico que deve ser utilizado para maximizar o potencial elétrico gerado pelo material piezelétrico.

\subsection{Formulação do Problema de OT para Sensores no Domínio Discretizado}

Para implementar o MOT de acordo com o fluxograma apresentado na Seção 3.4 é necessário transformar o problema de otimização, apresentado anteriormente, do domínio contínuo para o discretizado. Esta seção apresenta a função objetivo $L_{2}$ e a formulação do problema de otimização no domínio discretizado.

Primeiramente, apresenta-se a função $L_{2}$ escrita no domínio discretizado da seguinte maneira:

$$
L_{2}=\mathbf{F}_{\text {aux }}^{T} \breve{\mathbf{U}}_{2}
$$

onde $\mathbf{F}_{\text {aux }}$ (que tem o mesmo tamanho de $\breve{\mathbf{U}}_{2}$ ) é o vetor global de força auxiliar que contém valor igual a 1 somente no grau de liberdade mestre de potencial elétrico e valor nulo nos graus de liberdade restantes, e $\breve{\mathbf{U}}_{2}$ é o vetor global de deslocamentos mecânicos e potenciais elétricos, obtido a partir da solução do sistema linear da Eq. (5.3), sendo que $\breve{\mathbf{F}}_{2}$ é o vetor global que contém as pressões aplicadas na estrutura. 
No domínio discretizado, a distribuição de material e a orientação das fibras são discretizadas pelas variáveis topológicas $\mathbf{d}$ de acordo com a técnica de projeção, e pelas variáveis de orientação $\vartheta$ de acordo com a OMD, respectivamente. A polarização continua sendo representada pela variável de polarização $\varrho$. Assim, o problema de otimização para sensores piezocompósitos pode ser formulado, no domínio discretizado, da seguinte maneira:

$$
\begin{array}{ll}
\underset{\mathbf{d}, \boldsymbol{\varrho}, \boldsymbol{\vartheta}}{\operatorname{Maximizar}:} & L_{2}=\mathbf{F}_{\text {aux }}^{T} \breve{\mathbf{U}}_{2} \\
\text { sujeito à: } & \breve{\mathbf{K}} \breve{\mathbf{U}}_{2}=\breve{\mathbf{F}}_{2} \\
& s_{N P}=\left(\sum_{g=1}^{n_{\sigma}} s_{g}^{p_{\sigma}}\right)^{1 / p_{\sigma}} \geq 1 \\
& 0 \leq \mathbf{d} \leq 1 \\
& 0 \leq \boldsymbol{\varrho} \leq 1 \\
& 0 \leq \boldsymbol{\vartheta} \leq 1
\end{array}
$$

\subsection{Análise de Sensibilidades}

Como dito na Seção 3.4, a rotina de otimização MMA utiliza a primeira derivada da função objetivo e das restrições para encontrar os valores ótimos das variáveis de projeto a cada iteração. Assim, neste caso de sensores, é necessário obter a primeira derivada da função objetivo e da restrição de falha mecânica.

Derivando a função $L_{2}$ da Eq. (5.6) em função de uma variável genérica $v$, tem-se:

$$
\frac{\partial L_{2}}{\partial v}=\mathbf{F}_{a u x}^{T} \frac{\partial \breve{\mathbf{U}}_{2}}{\partial v}
$$

onde a variável $v$ é novamente utilizada para simplificar o desenvolvimento das derivadas, sendo que, posteriormente, ela deve ser substituída por $d, \varrho$ e $\vartheta . \mathbf{F}_{a u x}$ não depende das variáveis de projeto, e assim, sua derivada é igual a 0 .

A derivada do deslocamento $\breve{\mathbf{U}}_{2}$ é calculada derivando-se o sistema $\breve{\mathbf{K}}_{2}=\breve{\mathbf{F}}_{2}$ que fica:

$$
\frac{\partial \breve{\mathbf{U}}_{2}}{\partial v}=-\breve{\mathbf{K}}^{-1} \frac{\partial \breve{\mathbf{K}}}{\partial v} \breve{\mathbf{U}}_{2}
$$

já que $\partial \breve{\mathbf{F}}_{2} / \partial v=\mathbf{0}$, pois $\breve{\mathbf{F}}_{2}$ não depende das variáveis de projeto. A matriz $\breve{\mathbf{K}}$ pode ser invertida apenas uma vez e ser utilizada para todas as variáveis de projeto. Além disso, substituindo a Eq. (5.9) na Eq. (5.8) é possível utilizar o método adjunto para evitar a 
inversão da matriz $\breve{\mathbf{K}}$. Assim a Eq. (5.8) pode ser reescrita da seguinte maneira:

$$
\frac{\partial L_{2}}{\partial v}=-\breve{\mathbf{U}}_{a d j}^{T} \frac{\partial \breve{\mathbf{K}}}{\partial v} \breve{\mathbf{U}}_{2}
$$

onde $\breve{\mathbf{U}}_{a d j}$ é a solução de um sistema na forma:

$$
\breve{\mathbf{K}}_{a d j}=\mathbf{F}_{a u x}
$$

A derivada da restrição de falha mecânica é obtida substituindo-se a Eq. (5.9) na Eq. (3.76) e em seguida na Eq. (3.73), resultando em:

$$
\frac{\partial s_{N P}}{\partial v}=\operatorname{Aux}_{1}-\mathbf{F}_{\sigma}^{T} \breve{\mathbf{K}}^{-1} \frac{\partial \breve{\mathbf{K}}}{\partial v} \breve{\mathbf{U}}_{2}
$$

sendo que nesse caso, o método adjunto também pode ser utilizado, ou seja:

$$
\frac{\partial s_{N P}}{\partial v}=\operatorname{Aux}_{1}-\breve{\mathbf{U}}_{a d j_{\sigma}}^{T} \frac{\partial \breve{\mathbf{K}}}{\partial v} \breve{\mathbf{U}}_{2}
$$

onde

$$
\breve{\mathbf{K}}_{a d j_{\sigma}}=\mathbf{F}_{\sigma}
$$

O cálculo de $\operatorname{Aux}_{1}$ (Eq. (3.74)) e de $\frac{\partial \breve{\mathbf{K}}}{\partial v}$ foram apresentados na seção 3.5.

\subsection{Resultados}

Nesta seção, são apresentados resultados numéricos de dois exemplos do projeto otimizado de sensores piezocompósitos de casca com restrição de tensões mecânicas.

Para a otimização de orientação dos ângulos de fibras no caso 2, são adotados 12

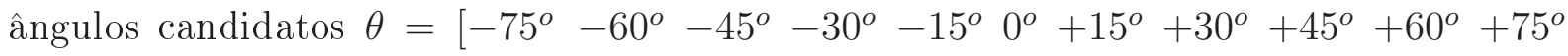
$\left.+90^{\circ}\right]$. Neste trabalho, atribui-se, inicialmente, valores iguais para todas as variáveis de topologia $d=0,5$ e de polarização $\varrho=0,6$. Não se utiliza o valor $\varrho=0,5$ para não gerar polarização nula logo no início do processo de otimização e criar instabilidades numéricas no método. O valor inicial para as variáveis de orientação deve ser igual a 0,5 de modo que nenhum ângulo candidato seja favorecido no início do processo de otimização. Para todos os exemplos apresentados, foram realizadas várias análises utilizando diferentes valores para as variáveis iniciais, que no final de cada análise, resultou em praticamente os mesmos resultados com valores de deslocamento muito próximos, com diferenças de no máximo $2 \%$. Assim, é possível afirmar que para os exemplos apresentados, chegou-se a 
soluções ótimas que estão muito próximas ao ótimo global.

Os valores dos coeficientes de penalização seguem a mesma abordagem utilizado no projeto de atuadores, ou seja, inicialmente, $p_{c}=p_{e}=p_{\vartheta}=1$, nas iterações 10, 20 e 30, $p_{e}$ e $p_{\vartheta}$ são incrementados em uma unidade, e nas iterações 30 e 40, $p_{c}$ e $p_{\vartheta}$ são incrementados em uma unidade. O raio utilizado na técnica de projeção é igual a $2 \mathrm{~mm}$.

Nas figuras de distribuição de material, são apresentados os valores das pseudodensidades, onde a cor preta representa o elemento cheio e a cor branca representa o elemento vazio. Valores intermediários das pseudodensidades são apresentadas em escalas de cinza. Nas figuras de polarização, os elementos em preto e branco possuem polarização positiva e negativa, respectivamente. Nas figuras das deformadas, as camadas em verde e azul representam as camadas piezelétricas com polarização negativa e positiva, respectivamente, e as camadas em vermelho e roxo representam as camadas de alumínio e de fibra de carbono, respectivamente. Nas figuras de otimização de orientação, são apresentadas as orientações ótimas em cada elemento por meio de uma reta.

Os exemplos apresentados neste capítulo tem o intuito de mostrar uma melhora incremental no projeto de sensores. Primeiramente mostra-se a necessidade de se incluir uma restrição de falha mecânica. Em seguida é apresentado um exemplo que mostra que o resultado ótimo depende do carregamento aplicado ou da restrição utilizada. Finalmente, para consolidar a metodologia proposta para sensores, são apresentados exemplos considerando, simultaneamente, a otimização de distribuição e de polarização de material piezelétrico e de orientação das fibras do material ortotrópico, sujeito à restrição de falha mecânica.

\subsection{Exemplo 1}

No primeiro exemplo, a estrutura analisada é um cilindro conforme apresentado na Fig. 5.2. O comprimento da estrutura é igual a $l=15 \mathrm{~mm}$ e o raio interno é igual a $r_{i}=20$ mm. Neste caso, é possível analisar somente um quarto da estrutura pois assume-se simetria geométrica, o que gera um espelhamento das variáveis (de topologia, polarização e orientação) e dos deslocamentos com relação aos planos $x-z$ e $y-z$. São estudados dois casos de empilhamento, substrato de alumínio coberto com uma camada de material piezelétrico, e um substrato composto de uma camada de alumínio e uma camada de fibra de carbono, cobertos por uma camada de material piezelétrico. As propriedades do alumínio, da fibra de carbono e do material piezelétrico utilizado podem ser encontrados 
no Apêndice C. A camada de substrato permanece fixa durante a otimização, ou seja, não é retirado material desta camada para evitar singularidades numéricas ao aplicar carregamento em elementos vazios. A espessura da camada piezelétrica é sempre igual a $t_{p}=0,2 \mathrm{~mm}$. A espessura da camada de substrato de alumínio é igual a $t_{s}=0,4 \mathrm{~mm}$. Já para o substrato composto, as duas camadas têm espessuras iguais a $t_{a}=t_{c}=0,2 \mathrm{~mm}$, ou seja, sua soma é igual a 0,4 $\mathrm{mm}$ para poder comparar os resultados deste empilhamento com os resultados do outro. O sentido de polarização do material piezelétrico segue a direção radial, sendo que a polarização positiva segue o sentido de dentro para fora do cilindro. É aplicada uma pressão na superfície interna do cilindro igual a $1 \mathrm{MPa}$.

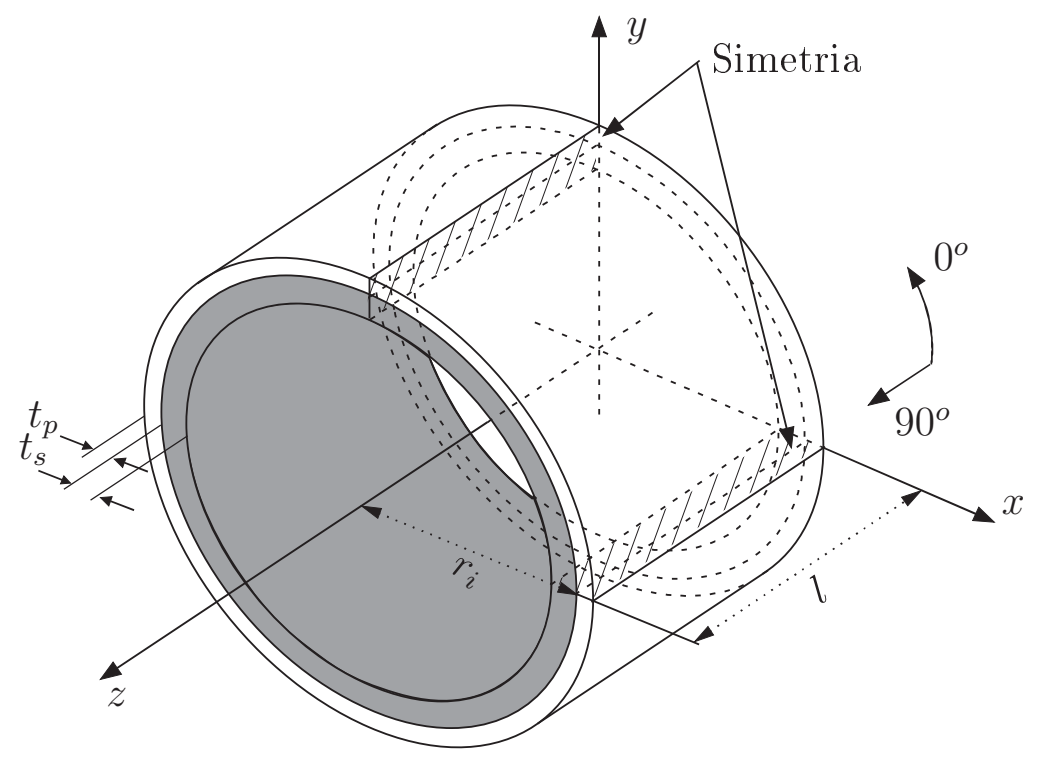

Figura 5.2: Domínio de projeto para o exemplo de sensor de casca piezocompósito. Camada interna é o substrato (alumínio ou alumínio/fibra de carbono) e a camada externa é de material piezelétrico.

A estrutura está engastada em todo o lado em que $z=0$. Entretanto, somente a camada de substrato está engastada, ou seja, a camada de material piezelétrico está apenas colada sobre o substrato e não é presa no engaste. Essa abordagem aproxima a modelagem de uma estrutura real, já que a fixação é feita apenas no substrato. Foi realizada uma análise de convergência do MEF para a estrutura simétrica e foi observada a convergência de potencial elétrico e de tensões mecânicas com uma discretização de 40 elementos na direção circunferencial e 20 elementos na direção do eixo $z$ em cada camada.

O primeiro caso estudado neste exemplo refere-se ao domínio inicial de projeto, com a camada piezelétrica totalmente cheia, e com polarização negativa para gerar potencial elétrico positivo nos eletrodos. Ou seja, nesse exemplo é feita somente uma análise da estrutura. Para medidas de comparação, esse caso passa a ser denominado de caso 0. 


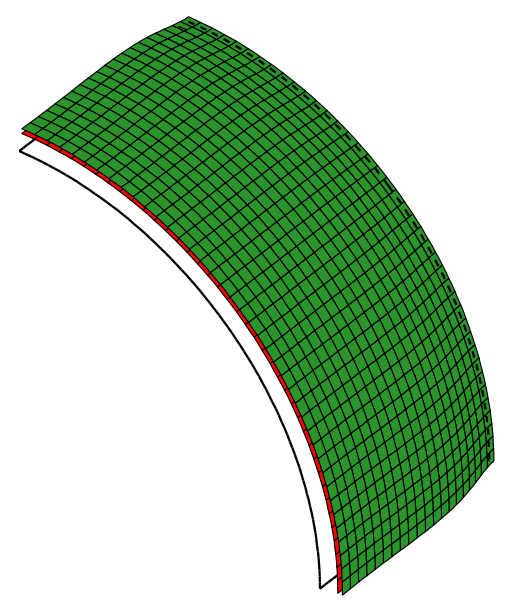

Figura 5.3: Forma deformada da estrutura para o caso 0 do projeto de sensores de casca, ou seja, sem otimização, com as camadas piezelétricas totalmente cheias e com polarização negativa.

A Fig. 5.3 apresenta a forma deformada da estrutura para o caso 0, cujo potencial elétrico é igual a 69,31 V. Analisando a distribuição das tensões, apresentada para as superfícies inferior e superior de cada camada, conforme Fig. 5.4, percebe-se que os valores de tensão mecânica estão respeitando os limites estabelecidos. A tensão máxima calculada na camada piezelétrica é igual a 49,30 MPa, praticamente igual à tensão limite de despolarização $\sigma_{d}$ que é igual a $50 \mathrm{MPa}$. Já a tensão máxima calculada na camada de alumínio é de 85,19 $\mathrm{MPa}$, bem abaixo do limite de escoamento de $\sigma_{y}=300 \mathrm{MPa}$. Ou seja, estamos partindo de uma configuração onde a restrição de falha é respeitada.

No próximo caso, é realizada somente as otimizações de topologia (distribuição de material) e de polarização para que se possa avaliar o comportamento da função objetivo sem a restrição de falha. Esse caso passa a ser denominado de caso 1. As Figs. 5.5(a) e 5.5(b) referem-se aos resultados de otimização de topologia e de polarização para esse caso e a Fig. 5.6 apresenta sua respectiva deformada.

O valor do potencial elétrico da estrutura otimizada é igual a 84,46 V, 21,86\% maior que no caso 0. Esse aumento no valor do potencial elétrico se justifica pela distribuição de material somente onde é necessário. Ou seja, nota-se pela Fig. 5.5(a) que nas regiões próximas ao engaste e à borda livre não deve haver material. O volume ótimo final da camada piezelétrica é igual a 50\% do total desta camada. Na Fig. 5.6 é apresentada a forma deformada da topologia final.

Analisando agora a distribuição das tensões, representado pela Fig. 5.7, é possível observar que há agora regiões na camada piezelétrica com tensões maiores que a tensão de despolarização. As tensões máximas calculadas nas camadas de alumínio e de material 


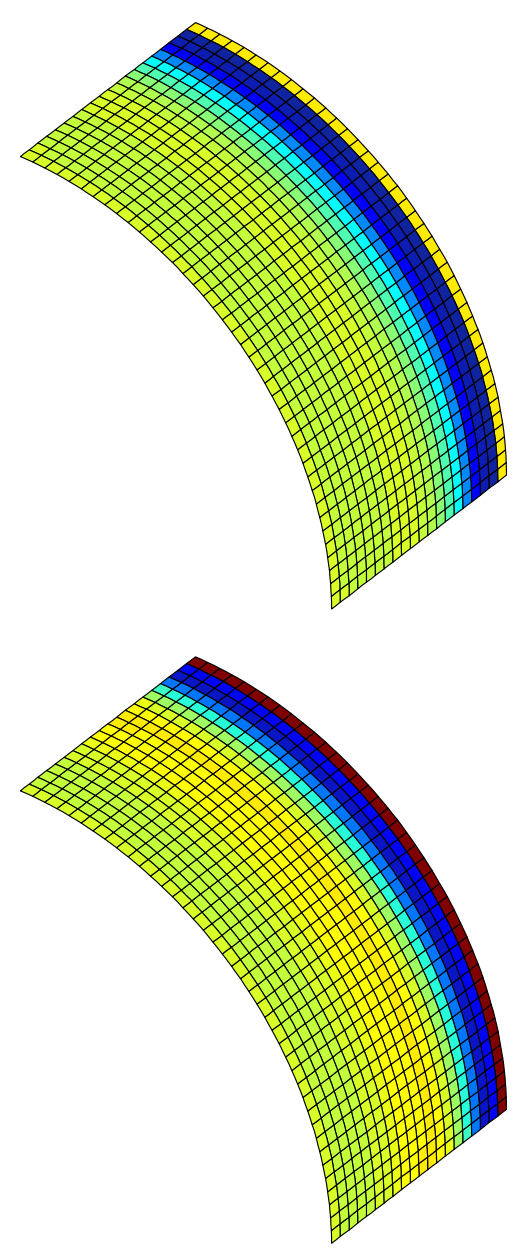

(a) Camada de alumínio Superfícies superior e inferior

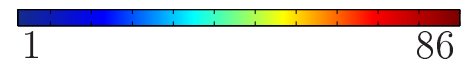

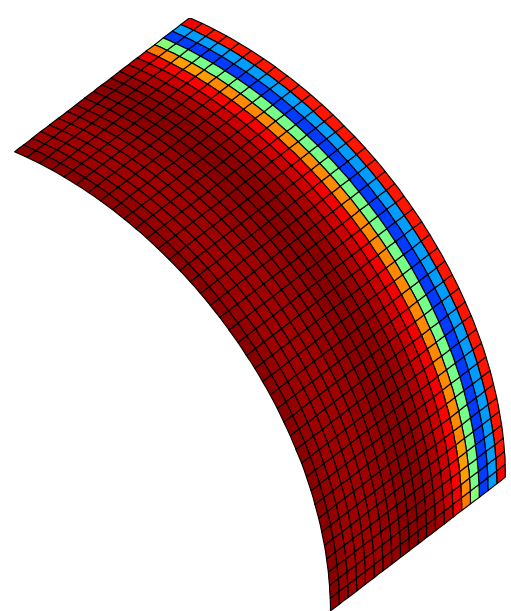

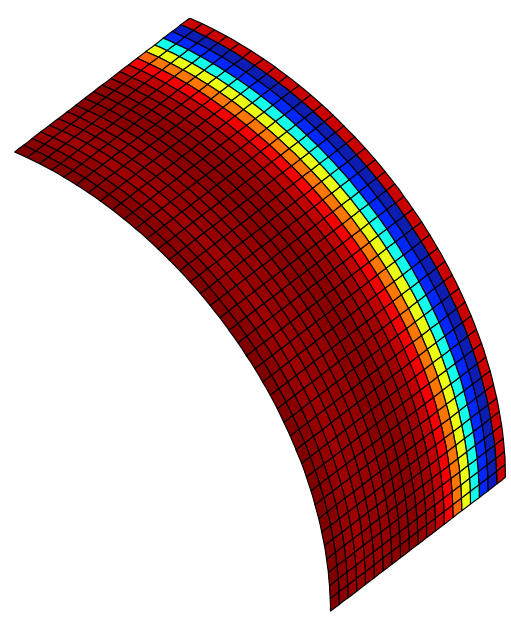

(b) Camada piezelétrica Superfícies superior e inferior

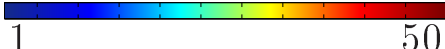

Figura 5.4: Distribuição das tensões de von Mises para o caso 0 do projeto de sensores de casca. Valores máximos: 85,16 MPa e 49,30 MPa para as camadas de alumínio e piezelétrica, respectivamente.

piezelétrico são iguais a 91,77 MPa e 57,08 MPa, respectivamente. Ou seja, apesar de ter um grande aumento no valor do potencial elétrico, a restrição de falha é desrespeitada.

Assim, para o próximo exemplo, denominado de caso 2, é realizada a mesma otimização do caso 1, considerando agora a restrição de falha mecânica. Como foi descrito anteriormente, a restrição de falha é implementada pela restrição de fator de segurança, que serve para normalizar os valores de tensão e nos dá uma ideia do quanto podemos aumentar ou diminuir o carregamento aplicado. Ao impor o fator de segurança como restrição, o valor mínimo calculado em toda a estrutura deve ser maior ou igual a 1.

As Figs. 5.8(a) e 5.8(b) apresentam os resultados de otimização de topologia e de 


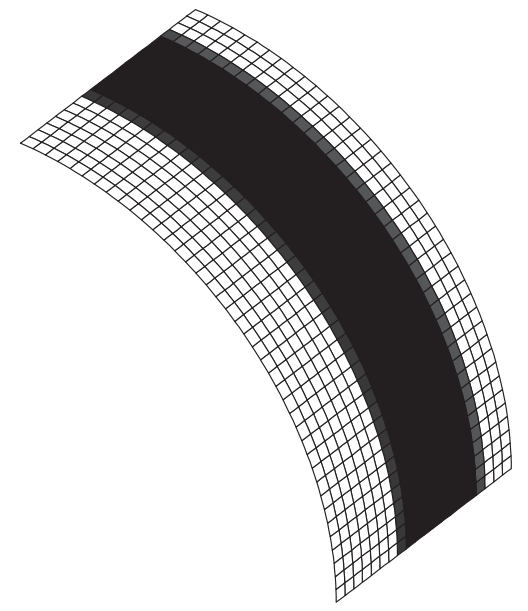

(a) Topologia ótima

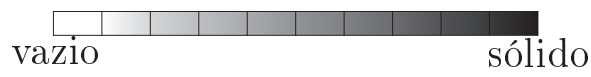

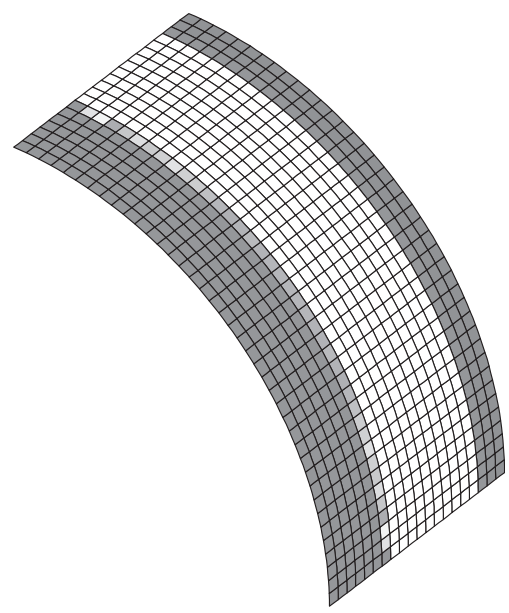

(b) Polarização ótima

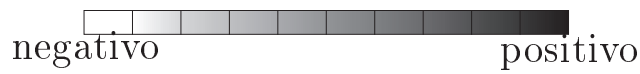

Figura 5.5: Resultado da otimização de topologia (a) e de polarização (b) da camada de material piezelétrico para o caso 1 do projeto de sensores de casca.

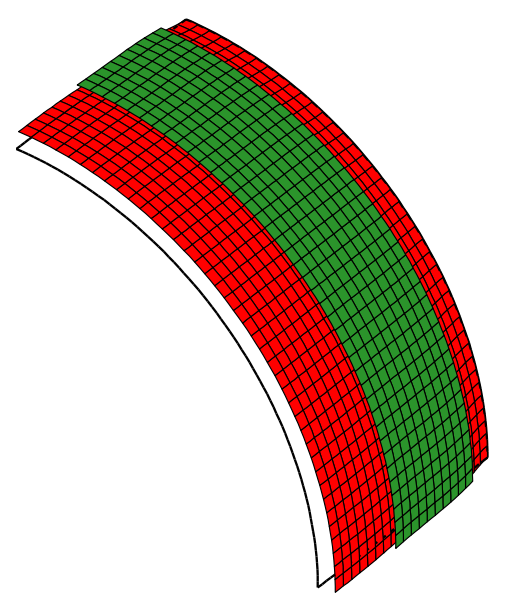

Figura 5.6: Forma deformada da estrutura para o caso 1 do projeto de sensores de casca.

polarização para o caso 2 e a Fig. 5.9 apresenta sua respectiva deformada.

O valor do potencial elétrico da estrutura otimizada é igual a $83,78 \mathrm{~V}, 20,88 \%$ maior que no caso 0 e apenas $0,8 \%$ menor que no caso 1 . A Fig. 5.9 mostra a forma deformada da estrutura e a Fig. 5.10 mostra as distribuições de tensão.

Nota-se qua a topologia ótima neste caso, ilustrado pela Fig. 5.8(a), abrange uma região maior que no caso 1 , com volume ótimo de material piezelétrico igual a 85,05\% do total da camada, sendo que apenas a região próxima ao engaste não possui material piezelétrico. Essa maior quantidade de material permitiu que a restrição de falha mecânica 


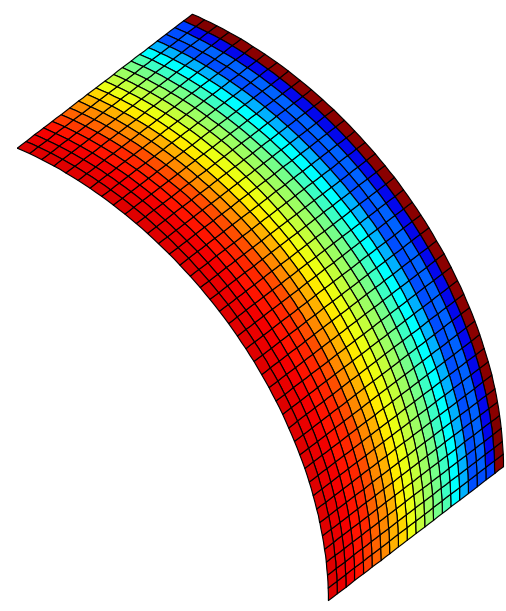

(a) Camada de alumínio Superfície superior

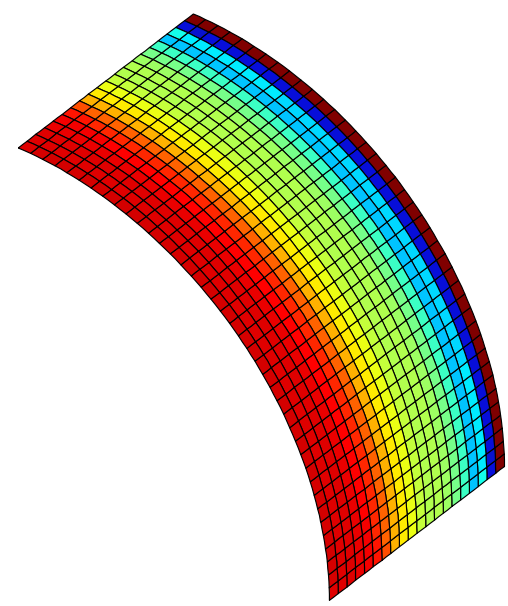

(c) Camada de alumínio Superfície inferior

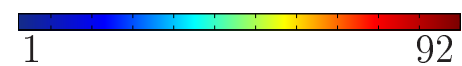

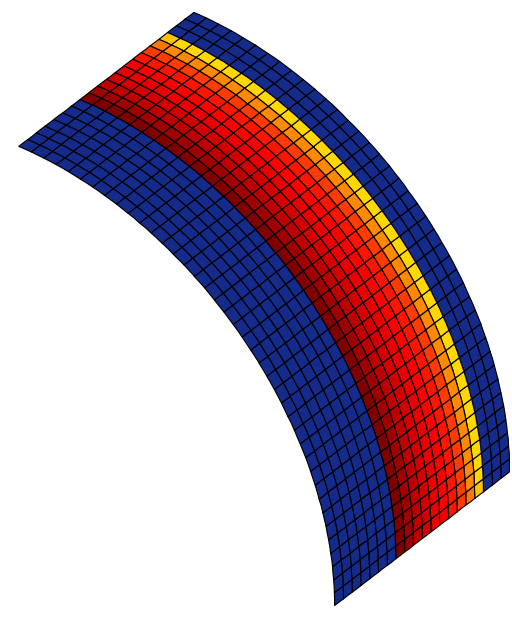

(b) Camada piezelétrica Superfície superior

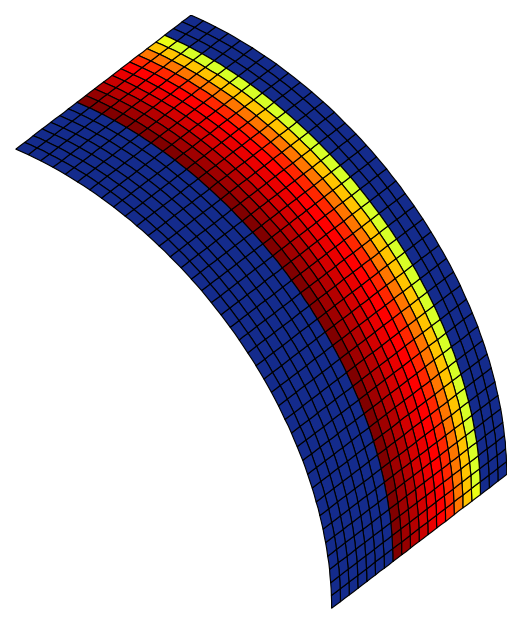

(d) Camada piezelétrica Superfície inferior

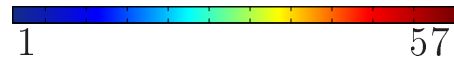

Figura 5.7: Distribuição das tensões de von Mises para o caso 1 do projeto de sensores de casca. Valores máximos: $91,77 \mathrm{MPa}$ e 57,08 MPa para as camadas de alumínio e piezelétrica, respectivamente.

voltasse a ser respeitada fazendo com que a estrutura se tornasse mais rígida. Analisando a distribuição de tensões da Fig. 5.10 pode-se observar que o maior valor de tensão mecânica fica ao longo da borda livre cujo valor é igual a 48,69 MPa. A tensão máxima calculada na camada de alumínio novamente tem valor bem abaixo do limite de escoamento, e é igual a 92,89 MPa.

O próximo exemplo (caso 3) considera agora um valor maior na restrição de falha mecânica de modo que o sensor não opere no limite. Foram testados os valores de 


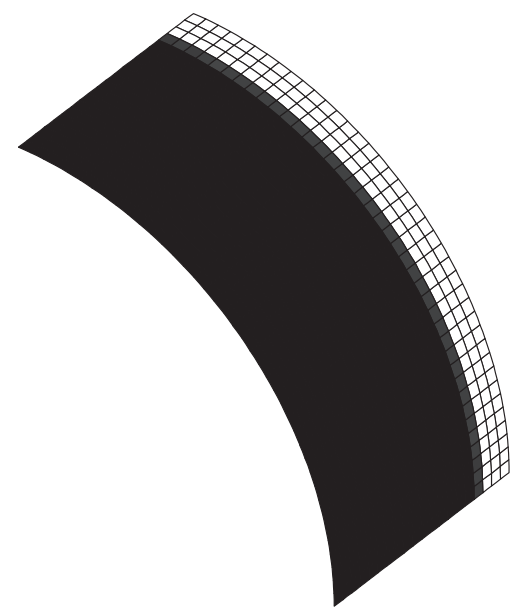

(a) Topologia ótima

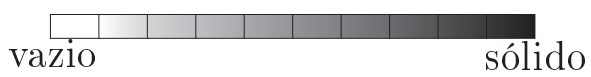

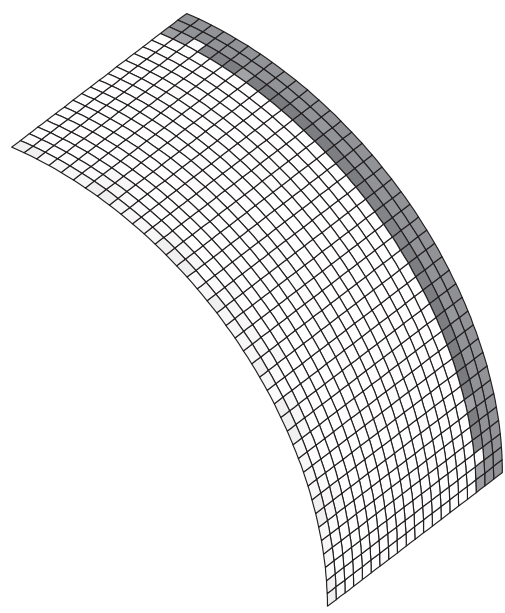

(b) Polarização ótima

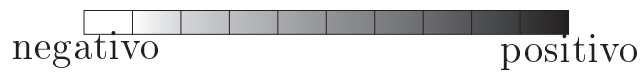

Figura 5.8: Resultado da otimização de topologia (a) e de polarização (b) da camada de material piezelétrico para o caso 2 do projeto de sensores de casca.

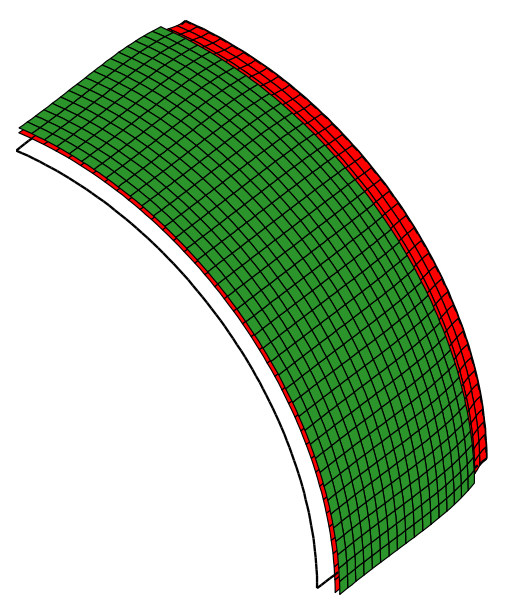

Figura 5.9: Forma deformada da estrutura para o caso 2 do projeto de sensores de casca.

restrição iguais a 1,5, 1,3 e 1,1. Entretanto, para essas 3 restrições, o resultado ótimo é uma estrutura sem camada piezelétrica, ou seja, uma topologia ótima de material piezelétrico vazia. Consequentemente, o valor do potencial elétrico para cada resultado é nulo. Por esses motivos, não são apresentadas nenhuma figura de otimização de topologia, de polarização, e nem de distribuição de tensões mecânicas.

O último exemplo (caso 4) considera a otimização de topologia, de polarização e de orientação de fibras, sujeitas a restrição de falha mecânica. Neste caso, parte do substrato de alumínio é substituído por uma camada de fibra de carbono. Ou seja, a sequência de empilhamento é alumínio/fibra de carbono/piezelétrico. Como foi dito anteriormente, a 


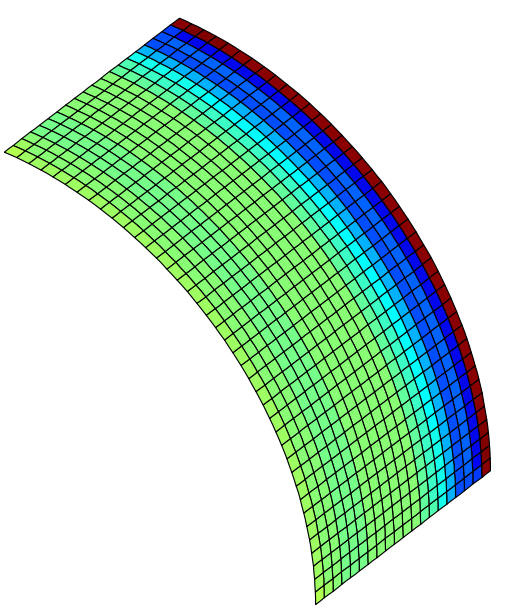

(a) Camada de alumínio Superfície superior

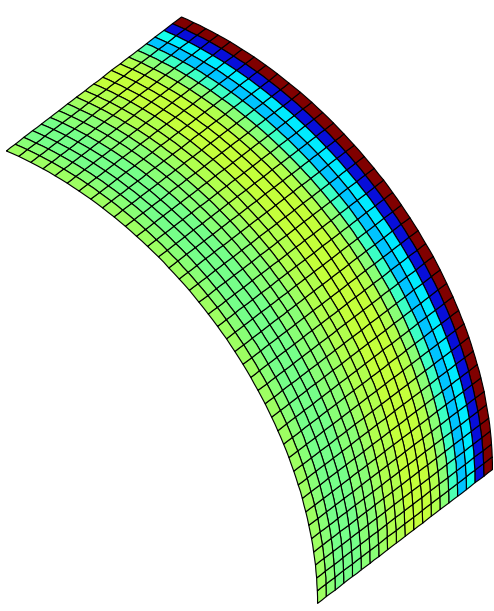

(c) Camada de alumínio Superfície inferior

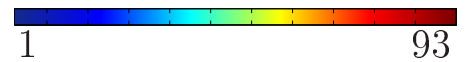

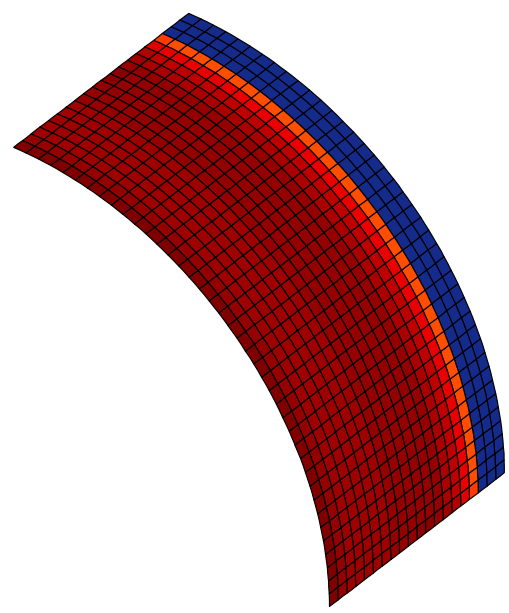

(b) Camada piezelétrica Superfície superior

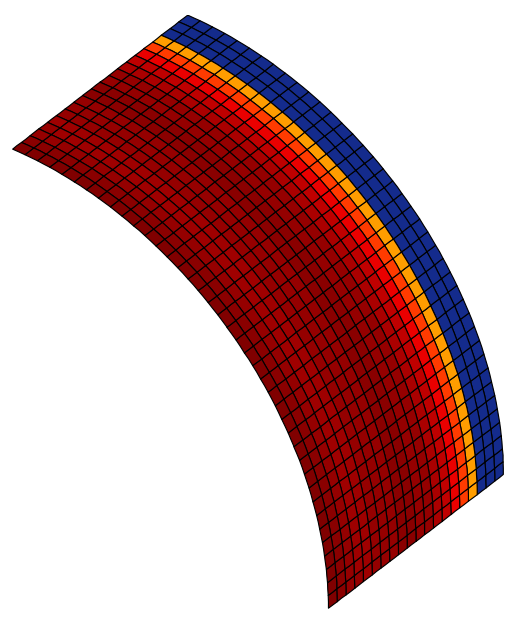

(d) Camada piezelétrica Superfície inferior

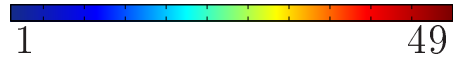

Figura 5.10: Distribuição das tensões de von Mises para o caso 2 do projeto de sensores de casca. Valores máximos: 92,89 $\mathrm{MPa}$ e 48,69 MPa para as camadas de alumínio e piezelétrica, respectivamente.

espessura da camada de alumínio reduz de 0,4 $\mathrm{mm}$ para 0,2 $\mathrm{mm}$ para dar espaço a camada de fibra de carbono, cuja espessura é igual a $0,2 \mathrm{~mm}$, que tem a função de alterar a rigidez do substrato, permitindo deformações maiores em algumas regiões e menores em outras, maximizando o potencial elétrico e reduzindo o valor das tensões.

As Figs. 5.11(a) e 5.11(b) apresentam os resultados de otimização de topologia e de polarização para o caso 4 e a Fig. 5.12(b) apresenta sua respectiva deformada. 


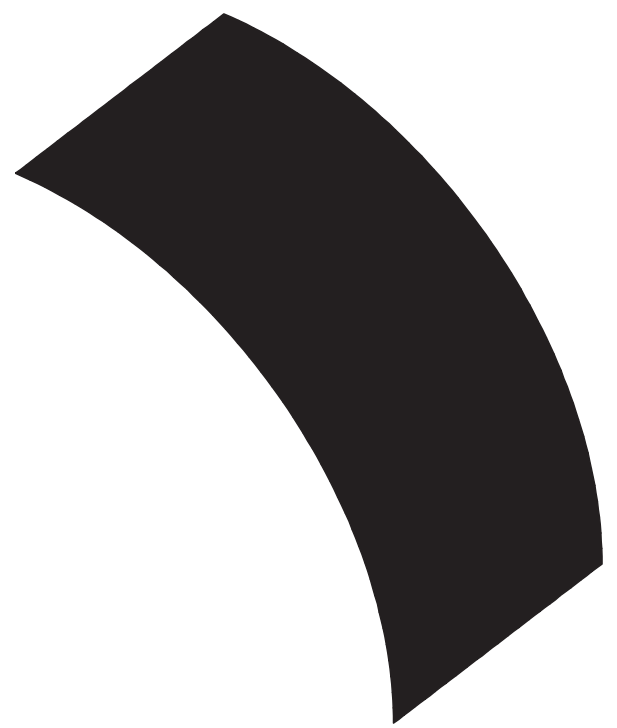

(a) Topologia ótima

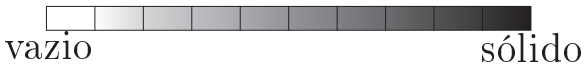

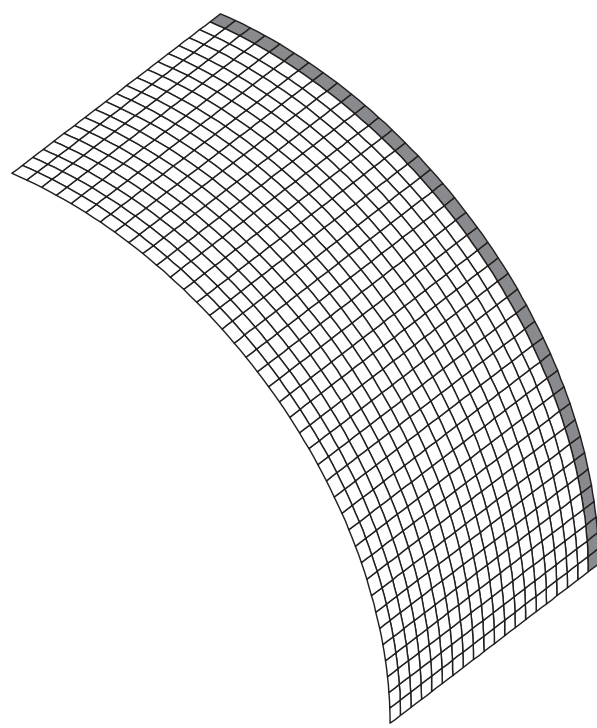

(b) Polarização ótima

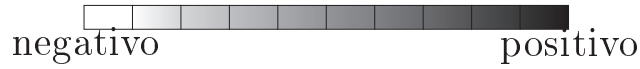

Figura 5.11: Resultado da otimização de topologia (a) e de polarização (b) da camada de material piezelétrico para o caso 4 do projeto de sensores de casca.

O valor do potencial elétrico da estrutura otimizada é igual a $88,53 \mathrm{~V}$, o maior de todos os casos. Nota-se qua a topologia ótima neste caso, ilustrado pela Fig. 5.11(a), é totalmente cheia. Entretanto, próximo ao engaste, a polarização é positiva pois as deformações nessa região são de compressão, enquanto que no resto da camada, a polarização é negativa pois as deformações são de tração. A Fig. 5.12(b) mostra a forma deformada da estrutura e a Fig. 5.13 mostra as distribuições de tensão mecânica.

Analisando a distribuição das tensões mecânicas no caso 4, os limites de tensão para todas as camadas foram obedecidas e, portanto, não apresenta falhas. As tensões máximas de von Mises calculadas nas camadas de alumínio e de material piezelétrico são iguais a 97,52 e 50,12 MPa, e a tensão máxima de Tsai-Wu para a camada de fibra de carbono é igual a 0,07. Assim, a otimização de orientação de fibras junto com a otimização de distribuição e da polarização de material piezelétrico, além de aumentar o valor dos potenciais elétricos gerados, os valores de tensão são reduzidos

A Tab. 5.1 apresenta os valores de potencial elétrico gerado $(\Delta \phi)$, de tensão máxima de von Mises na camada piezelétrica $\left(\sigma_{v m_{\text {max }}}^{\text {piezo }}\right)$ e na camada de alumínio $\left(\sigma_{v m_{\text {max }}}^{A l}\right)$, de tensão máxima de Tsai-Wu na camada de fibra de carbono $\left(\sigma_{t w_{\max }}^{F C}\right)$ e de volume final da camada piezelétrica otimizada $\left(V_{o t}\right)$ para todos os casos apresentados. 


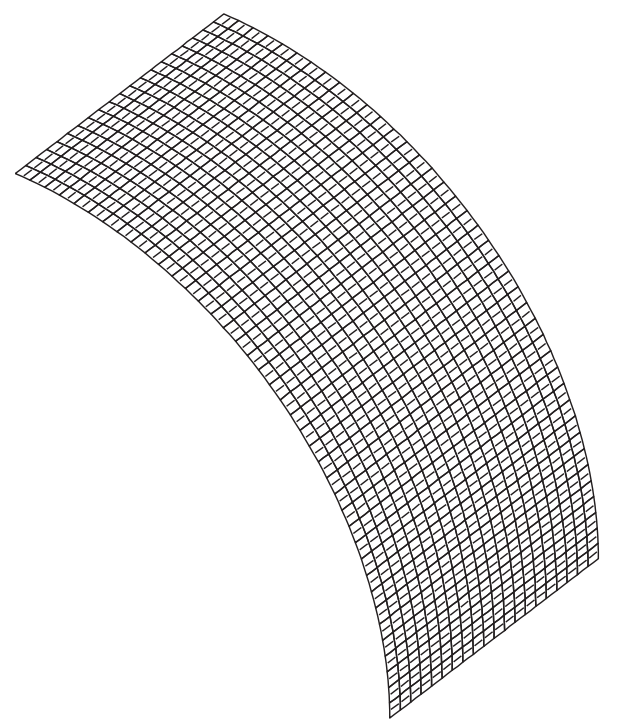

(a) Camada de fibra de carbono

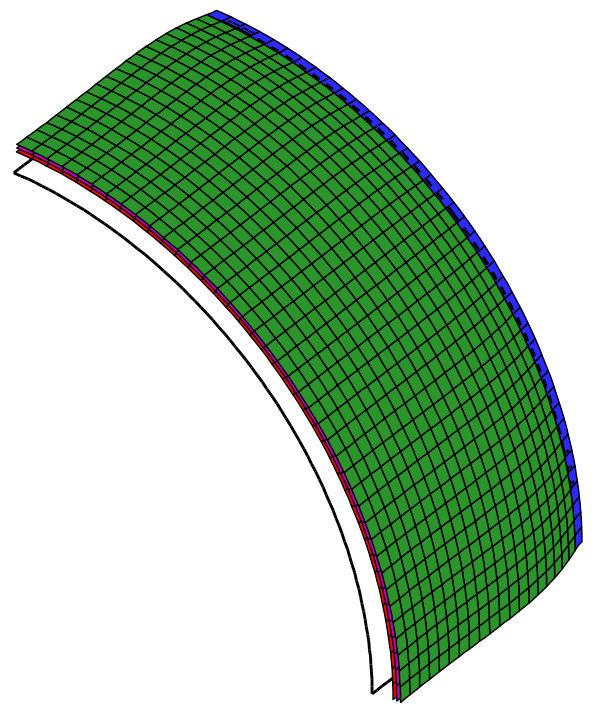

(b) Forma deformada

Figura 5.12: Resultado da otimização de orientação de fibras (a) e forma deformada (b) do resultado pós-processado para o caso 4 do projeto de sensores de casca. Em vermelho está representada a camada de alumínio, e em azul e verde está representada a camada piezelétrica com polarização positiva e negativa, respectivamente.

Tabela 5.1: Dados dos resultados apresentados para o exemplo de sensores de casca.

\begin{tabular}{cccccc}
\hline & $\Delta \phi$ & $\sigma_{v m_{\max }}^{\text {piezo }}$ & $\sigma_{v m_{\max }}^{A l}$ & $\sigma_{\text {tw }}^{F C}$ & $\begin{array}{c}V_{\text {ot }} \\
\mathrm{MPa}\end{array}$ \\
\hline caso 0 & 61,31 & 49,30 & 85,19 & - & - \\
caso 1 & 84,46 & 57,08 & 91,77 & - & 50 \\
caso 2 & 83,78 & 48,69 & 92,89 & - & 85,05 \\
caso 3 & - & - & - & - & - \\
caso 4 & 88,53 & 50,12 & 97,52 & 0,07 & 100 \\
\hline
\end{tabular}

\section{$5.7 \quad$ Exemplo 2}

No segundo exemplo estudado, a estrutura analisada é uma placa quadrada de lados $l=50 \mathrm{~mm}$, conforme apresentado na Fig. 5.14. São estudados dois casos de empilhamento, substrato de alumínio coberto com uma camada de material piezelétrico, e um substrato composto de uma camada de alumínio e uma camada de fibra de carbono, cobertos por uma camada de material piezelétrico. As propriedades do alumínio, da fibra de carbono e do material piezelétrico utilizado podem ser encontrados no Apêndice C, nas Tabs. C.1, C.5 e C.3, respectivamente. A camada de substrato permanece fixa durante a otimização, ou seja, não é retirado material desta camada para evitar singularidades numéricas ao aplicar carregamento em elementos vazios. A espessura da camada piezelétrica é sempre 


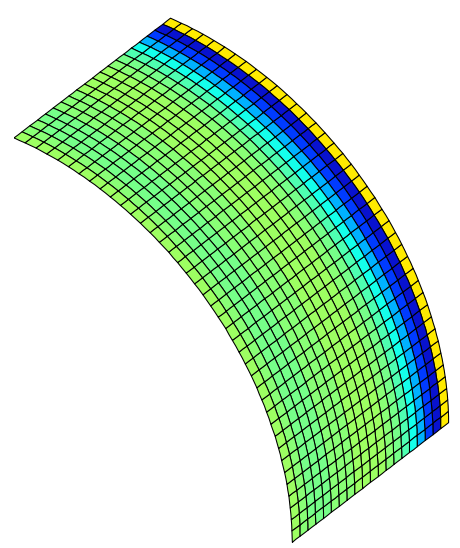

(a) Camada de alumínio Superfície superior

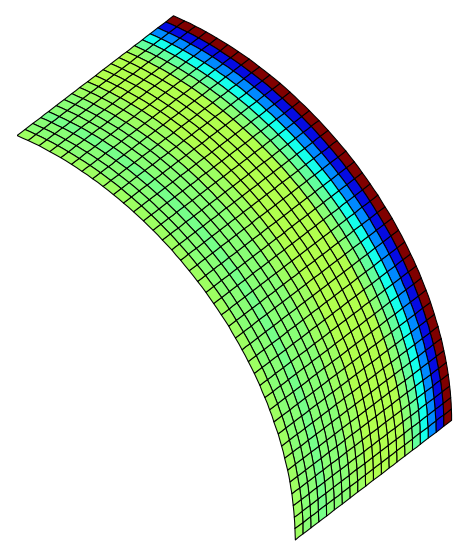

(d) Camada de alumínio Superfície inferior

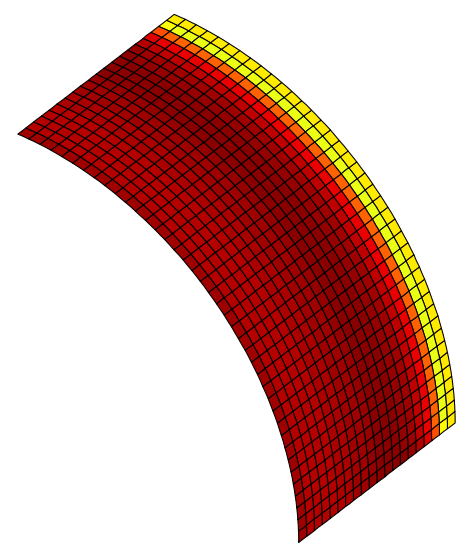

(b) Camada de fibra de carbono - Superfície superior

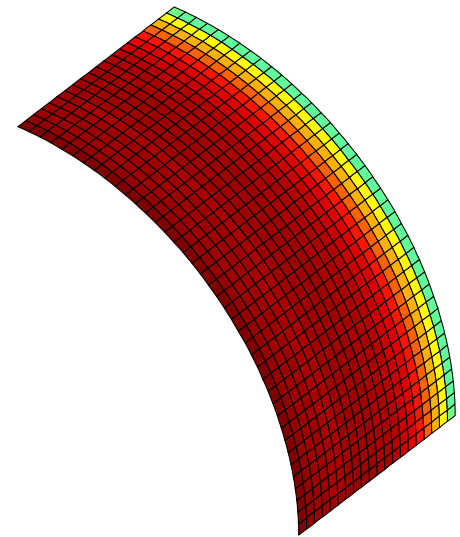

(e) Camada de fibra de carbono - Superfície inferior

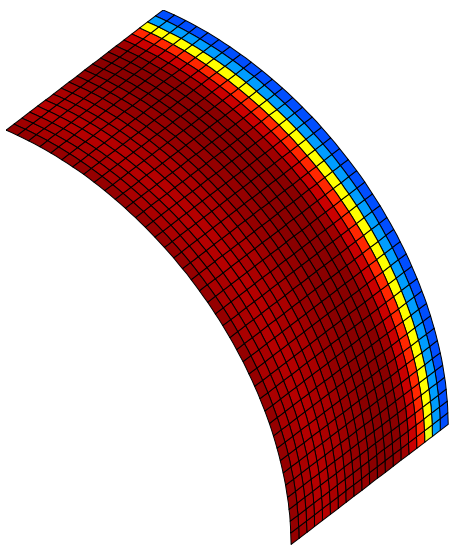

(c) Camada piezelétrica Superfície superior

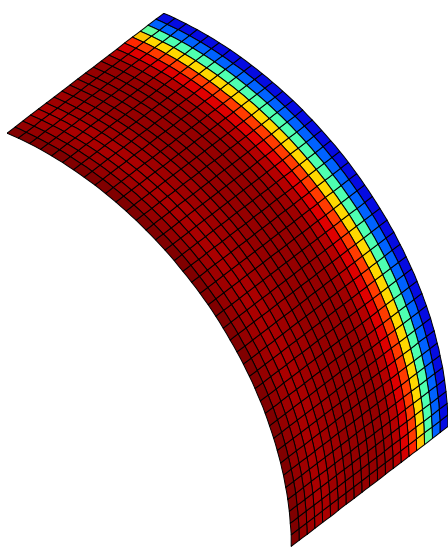

(f) Camada piezelétrica Superfície inferior

0 98

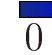

0,07

50

Figura 5.13: Distribuição das tensões de Tsai-Wu da camada de fibra de carbono e de von Mises das camadas de alumínio e de material piezelétrico para o caso 4 do exemplo 1 de coletores de energia. Valores de tensão de von Mises em MPa.

igual a $t_{p}=0,2 \mathrm{~mm}$. No primeiro caso de estudo, a espessura da camada de substrato de alumínio é igual a $t_{s}=0,4 \mathrm{~mm}$. Já para o substrato composto, as duas camadas têm espessuras iguais a $t_{a}=t_{c}=0,2 \mathrm{~mm}$, ou seja, sua soma é igual a 0,4 mm. O sentido de polarização do material piezelétrico segue a direção da espessura da placa, ou seja, o eixo $y$, e o sentido de polarização positiva segue o mesmo sentido positivo de $y$. É aplicada uma pressão na superfície inferior da placa igual a 0,05 MPa.

A estrutura está engastada em todos os lados. Entretanto, somente a camada de 


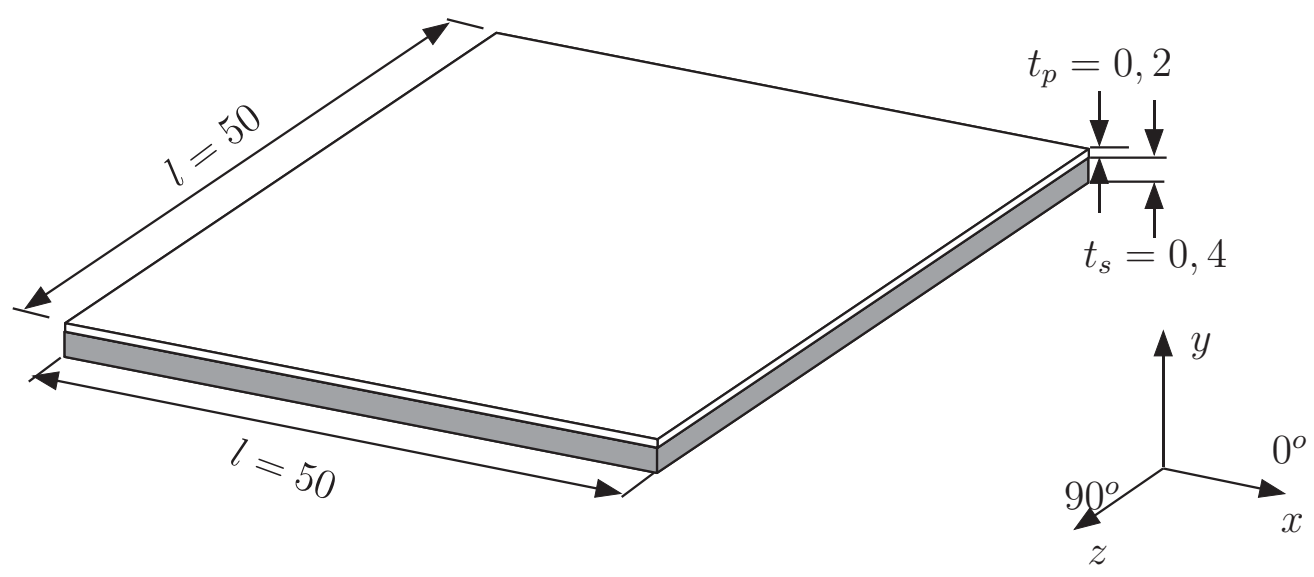

Figura 5.14: Domínio de projeto para o exemplo de sensor de placa piezocompósito. Camada inferior é o substrato (alumínio ou alumínio/fibra de carbono) e a camada superior é de material piezelétrico.

substrato está engastada, ou seja, a camada de material piezelétrico está apenas colada sobre o substrato e não é presa no engaste. Essa abordagem aproxima a modelagem de uma estrutura real, já que a fixação é feita apenas no substrato. Foi realizada uma análise de convergência do MEF para a estrutura simétrica e foi observada a convergência dos valores de potencial elétrico e de tensões mecânicas com uma discretização de 30x30 elementos em cada camada.

O primeiro caso estudado refere-se ao domínio inicial de projeto, com a camada piezelétrica totalmente cheia, e com polarização negativa para gerar potencial elétrico positivo nos eletrodos. Ou seja, nesse exemplo é feita somente uma análise da estrutura. Para medidas de comparação, esse caso passa a ser denominado de caso 0.

A Fig. 5.15 apresenta a forma deformada da estrutura para o caso 0, cujo potencial elétrico é igual a 3,40 V. Entretanto, analisando a distribuição das tensões, apresentada para as superfícies inferior e superior de cada camada conforme Fig. 5.16, percebe-se que existem regiões da camada piezelétrica que apresentam tensões maiores do que o limite tolerado. A tensão máxima calculada na camada piezelétrica é igual a 86,62 $\mathrm{MPa}$, enquanto que a tensão limite de despolarização $\sigma_{d}$ é de $50 \mathrm{MPa}$. Já a tensão máxima calculada na camada de alumínio é de 113,18 MPa, 62\% abaixo do limite de escoamento de $\sigma_{y}=300 \mathrm{MPa}$.

No próximo caso estudado, denominado de caso 1, é realizada a otimização de distribuição e de polarização do material piezelétrico sobre o substrato de alumínio, considerando a restrição de falha mecânica. As Figs. 5.17(a) e 5.17(b) apresentam os resultados de otimização de topologia e de polarização para o caso 1 e a Fig. 5.18 apresenta sua respectiva deformada. 


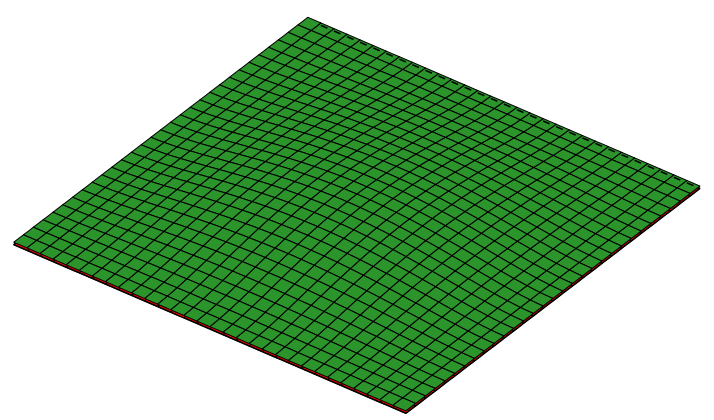

Figura 5.15: Forma deformada da estrutura para o caso 0 do projeto de sensores de placa, ou seja, sem otimização, com as camadas piezelétricas totalmente cheias e com polarização negativa.

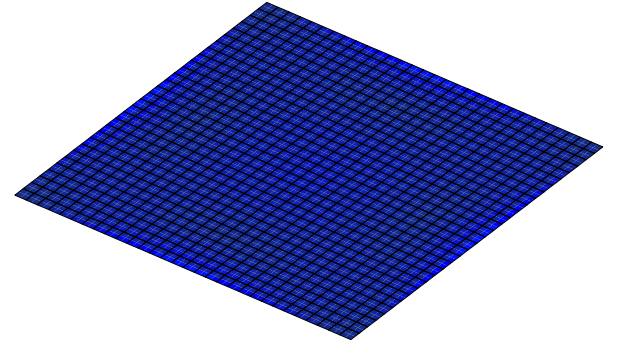

(a) Camada de alumínio - Superfície superior

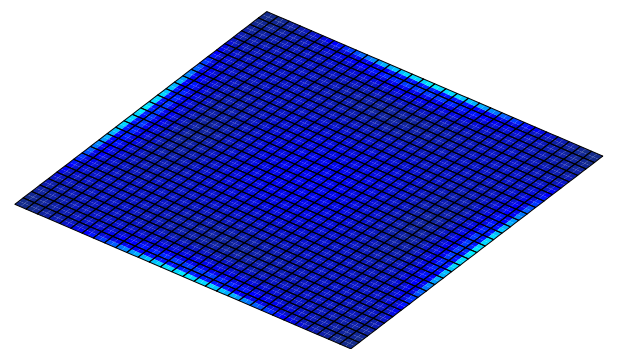

(c) Camada de alumínio - Superfície inferior

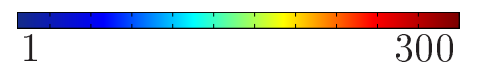

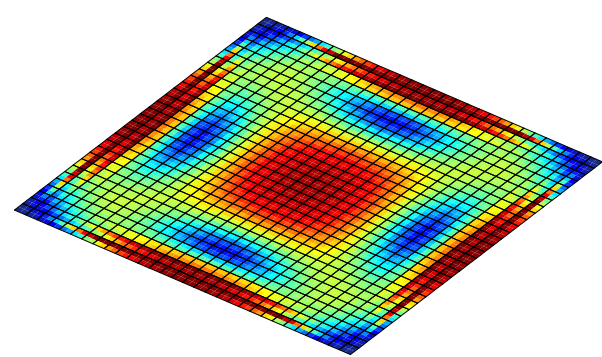

(b) Camada piezelétrica - Superfície superior

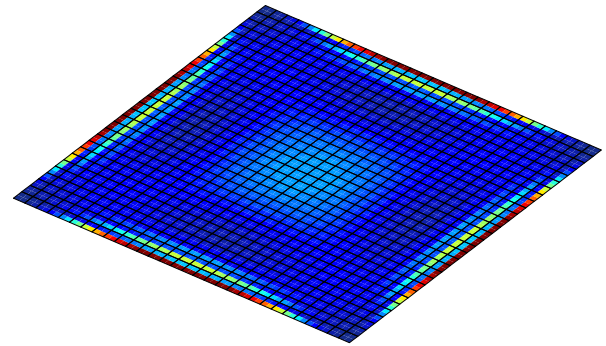

(d) Camada piezelétrica - Superfície inferior

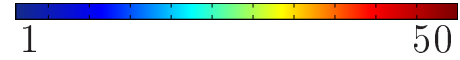

Figura 5.16: Distribuição das tensões de von Mises para o caso 0 do projeto de sensores de placa. Valores em MPa.

O valor do potencial elétrico da estrutura otimizada é igual a 69,03 V, mais de 20 vezes maior que no caso 0. Esse grande aumento no valor do potencial elétrico é devido à diferença de polarização na mesma camada de material piezelétrico, pois existem regiões onde a deformada é por tração e outras regiões que a deformada é por compressão. Assim, foi distribuído material piezelétrico com polarização negativa (em verde) na região central e com polarização negativa nas regiões próximas aos contos da placa para aproveitar 


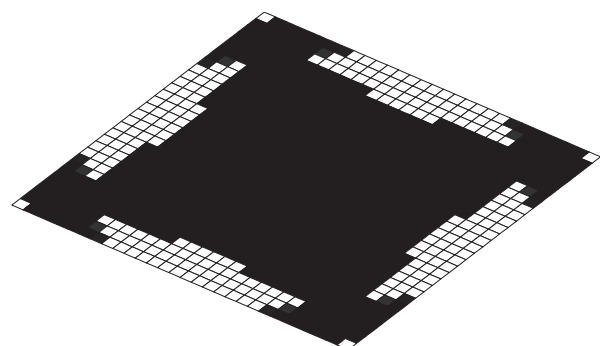

(a) Topologia ótima

vazio sólido

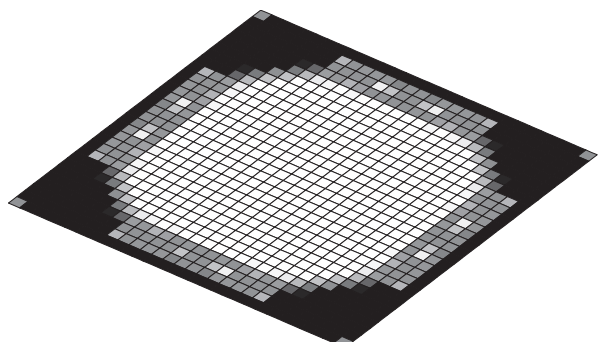

(b) Polarização ótima

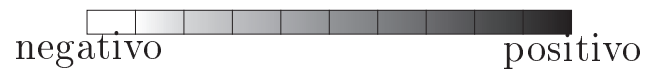

Figura 5.17: Resultado da otimização de topologia (a) e de polarização (b) da camada de material piezelétrico para o caso 1 do projeto de sensores de placa.

melhor essas deformações. A quantidade de volume na estrutura final é igual a $76 \%$ do volume total permitido na camada. A Fig. 5.18 mostra a forma deformada da estrutura e a Fig. 5.19 mostra as distribuições de tensão.

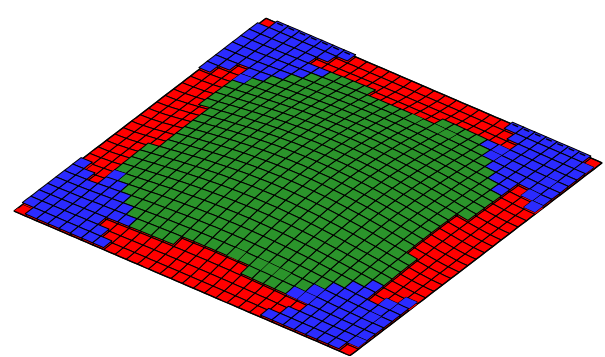

Figura 5.18: Forma deformada da estrutura para o caso 1 do projeto de sensores de placa. A camada de alumínio está representada na cor vermelha e a camada piezelétrica está representada nas cores azul e verde, com polarização positiva e negativa, respectivamente.

Analisando a distribuição das tensões no caso 1, é possível observar que ainda há regiões na camada piezelétrica com valores de tensões mecânica que estão acima do limite de despolarização $\left(\sigma_{d}=50 \mathrm{MPa}\right)$. As tensões máximas calculadas nas camadas de alumínio e de material piezelétrico são iguais a 138,67 e 57,21 MPa, respectivamente. Ou seja, mesmo considerando restrição de falha, nesse problema, para o valor de pressão utilizada, não foi possível atingir uma topologia que respeitasse o limite de despolarização do material piezelétrico.

Assim, no próximo exemplo, deseja-se verificar se é possível chegar a uma topologia que atenda os limites de tensão. Para isso, parte do substrato é substituído por um outro material. No caso, a fibra de carbono, que possui alta rigidez na direção longitudinal das fibras, e baixa rigidez na direção transversal. Assim, a ideia é que a otimização de 


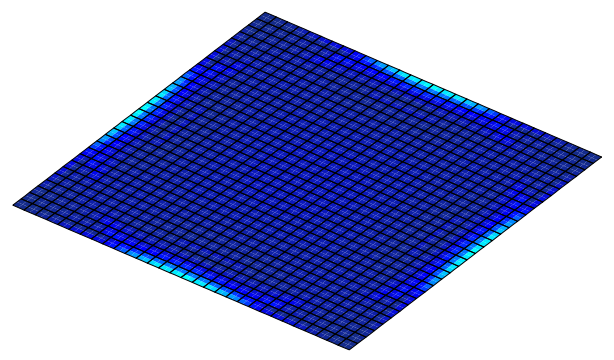

(a) Camada de alumínio - Superfície superior

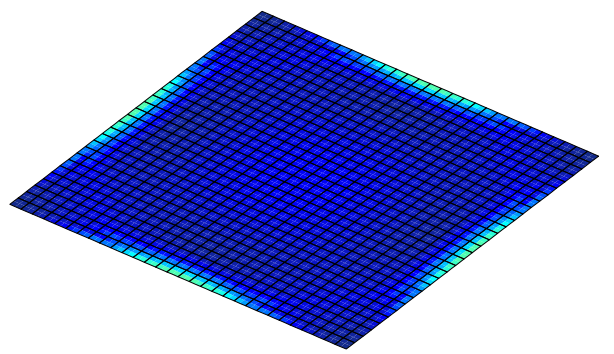

(c) Camada de alumínio - Superfície inferior

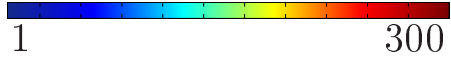

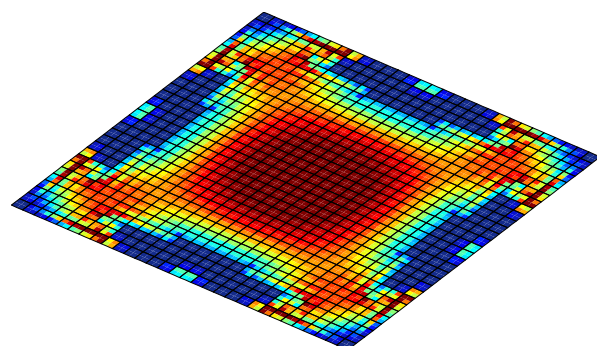

(b) Camada piezelétrica - Superfície superior

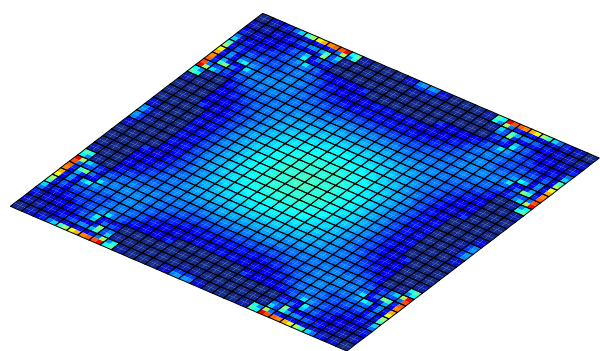

(d) Camada piezelétrica - Superfície inferior

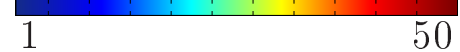

Figura 5.19: Distribuição das tensões de von Mises para o caso 1 do projeto de sensores de placa. Valores em MPa.

orientação de fibras escolha adequadamente os ângulos das fibras para que em regiões de maior tensão mecânica, a camada de fibra de carbono fique mais rígida, reduzindo o valor da tensão mecânica no material piezelétrico. Nas regiões com baixas tensões, a camada de fibra de carbono pode ficar mais flexível para que o material piezelétrico gere mais potencial elétrico. Como foi dito anteriormente, a espessura da camada de alumínio reduz de 0,4 $\mathrm{mm}$ para 0,2 $\mathrm{mm}$ para dar espaço a camada de fibra de carbono, cuja espessura é igual a $0,2 \mathrm{~mm}$.

As Figs. 5.20(a) e 5.20(b) apresentam os resultados de otimização de topologia e de polarização para o caso 2 e a Fig. 5.21 apresenta sua respectiva deformada.

O valor do potencial elétrico da estrutura otimizada é igual a $67,16 \mathrm{~V}$, um pouco menor que no caso 1. Entretanto, a pequena redução no valor do potencial elétrico obtido no caso 2 se justifica pelo fato de que, agora, as tensões mecânicas na estrutura obedecem aos limites de cada material. A quantidade de volume na estrutura final é igual a 82,27\% do volume total permitido na camada. A Fig. 5.21 mostra a forma deformada da estrutura e a Fig. 5.22 mostra as distribuições de tensão. 


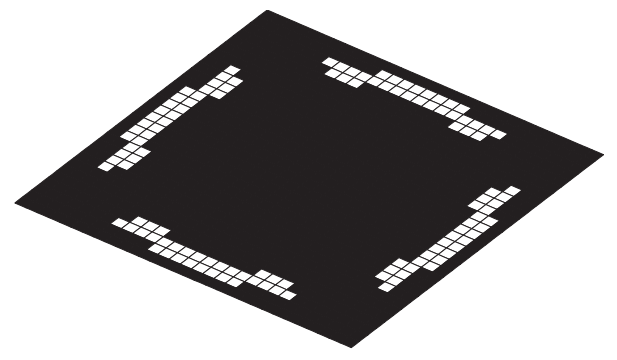

(a) Topologia ótima

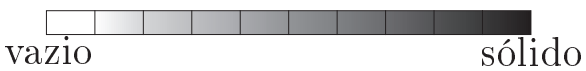

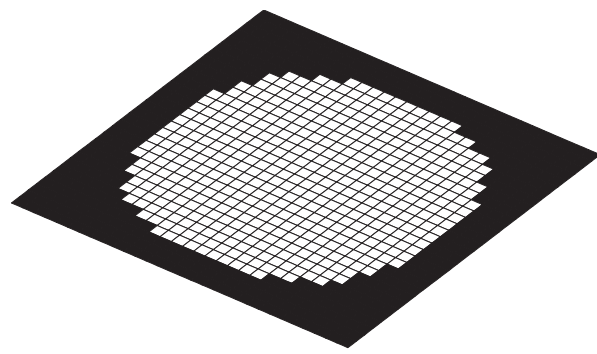

(b) Polarização ótima

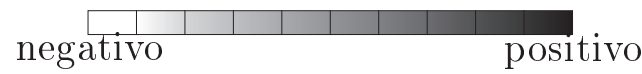

Figura 5.20: Resultado da otimização de topologia (a) e de polarização (b) da camada de material piezelétrico para o caso 2 do projeto de sensores de placa.

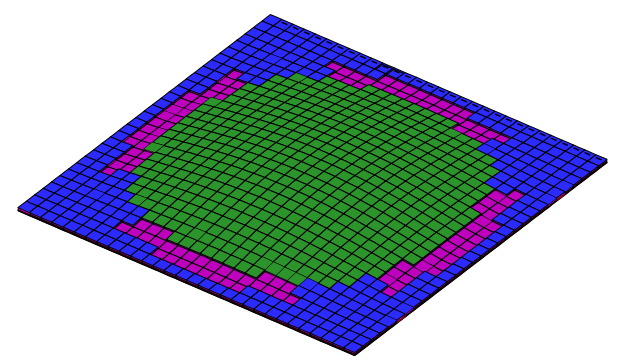

Figura 5.21: Forma deformada da estrutura para o caso 2 do projeto de sensores de placa. A camada de fibra de carbono está representada na cor roxa e a camada piezelétrica está representada nas cores azul e verde, com polarização positiva e negativa, respectivamente.

Analisando a distribuição das tensões no caso 2, é possível observar que os limites de tensão para todas as camadas foram obedecidas, e portanto, não apresenta falhas. As tensões máximas de von Mises calculadas nas camadas de alumínio e de material piezelétrico são iguais a 97,47 e 49,93 MPa, e a tensão máxima de Tsai-Wu para a camada de fibra de carbono é igual a 0,05. Assim, a otimização de orientação de fibras junto com a otimização de distribuição e da polarização de material piezelétrico, apesar de ter reduzido o valor do potencial elétrico gerado, garante que os limites de tensões mecânicas são obedecidos.

A Tab. 5.2 apresenta os valores de potencial elétrico gerado $(\Delta \phi)$, de tensão máxima de von Mises na camada piezelétrica $\left(\sigma_{v m_{\text {max }}}^{\text {piezo }}\right)$ e na camada de alumínio $\left(\sigma_{v m_{\text {max }}}^{A l}\right)$, de tensão máxima de Tsai-Wu na camada de fibra de carbono $\left(\sigma_{t w \text { max }}^{F C}\right)$ e de volume final da camada piezelétrica otimizada $\left(V_{o t}\right)$ para todos os casos apresentados. 


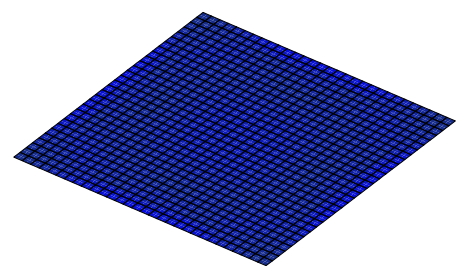

(a) Camada de alumínio Superfície superior

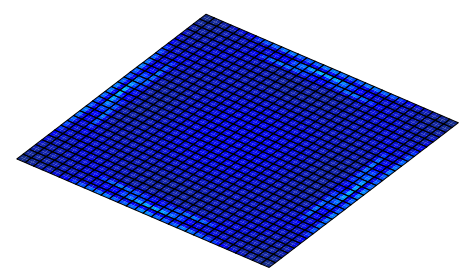

(d) Camada de alumínio Superfície inferior

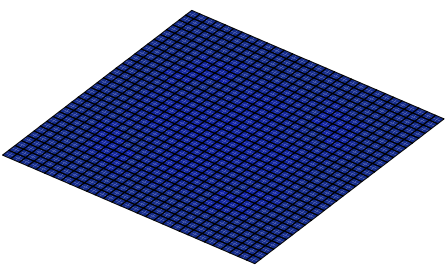

(b) Camada de fibra de carbono - Superfície superior

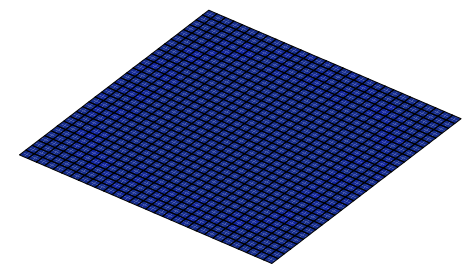

(e) Camada de fibra de carbono - Superfície inferior

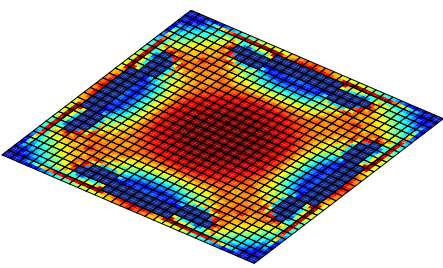

(c) Camada piezelétrica Superfície superior

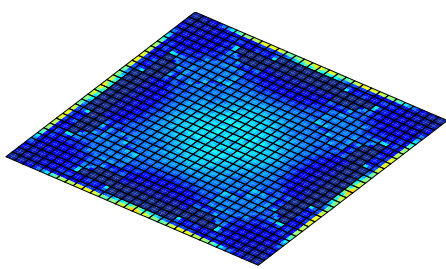

(f) Camada piezelétrica Superfície inferior
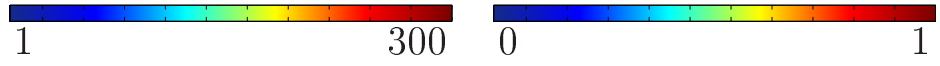

1

50

Figura 5.22: Distribuição das tensões de Tsai-Wu da camada de fibra de carbono e de von Mises das camadas de alumínio e de material piezelétrico para o caso 2 de coletores de energia de placa. Valores de tensão de von Mises em MPa.

Tabela 5.2: Dados dos resultados apresentados para o exemplo de sensores de casca.

\begin{tabular}{|c|c|c|c|c|c|}
\hline & $\begin{array}{c}\Delta \phi \\
\mathrm{V}\end{array}$ & $\begin{array}{c}\sigma_{v m_{\max }}^{\text {piezo }} \\
\mathrm{MPa}\end{array}$ & $\begin{array}{c}\sigma_{v m_{\text {max }}}^{A l} \\
\mathrm{MPa}\end{array}$ & $\sigma_{t w_{\max }}^{F C}$ & $\begin{array}{c}V_{o t} \\
\%\end{array}$ \\
\hline caso 0 & 3,4 & 86,62 & 113,18 & - & - \\
\hline caso 1 & 69,03 & 57,21 & 138,67 & - & 76 \\
\hline caso 2 & 67,16 & 49.93 & 97,47 & 0,05 & 82,27 \\
\hline
\end{tabular}

\subsection{Discussão}

Assim, é possível concluir que as tensões mecânicas não podem ser desprezadas no projeto de sensores, já que a amplitude do carregamento aplicado influencia na topologia ótima e no valor resultante de tensão elétrica. Foi observado que a camada crítica é sempre a camada de material piezelétrico, pois sua tensão de despolarização é muito baixa.

A restrição de falha mecânica se mostrou eficaz ao manter o valor máximo da tensão de von Mises na camada de material piezelétrico dentro dos limites impostos no problema, como pode ser visto comparando os casos 1 e 2. No caso 3, ao aumentar o valor da restrição, o valor máximo da tensão de von Mises ficou ainda menor, entretanto, o valor 
da tensão elétrica de saída também foi reduzida, configurando numa solução pior que no caso 2. Assim, sugere-se que a restrição de falha mecância seja utilizada sempre no limite, ou seja, o valor mínimo do fator de segurança deve ser maior ou igual a 1.

A otimização da orientação de fibras se mostrou como um parâmetro importante no projeto de sensores, já que foi possível aumentar o valor do potencial elétrico gerado no exemplo dos sensor de casca, mantendo os valores de tensão abaixo dos limites dos materiais.

Além disso, restrições de volume não devem ser utilizadas juntamente com restrições de tensão, já que uma topologia totalmente cheia de material pode-se configurar como uma estrutura com menor valor de tensões. 


\section{PROJETO DINÂMICO DE COLETORES DE ENERGIA PIEZOCOMPÓSITOS DE CASCA COM RESTRIÇÃO DE TENSÃO}

Neste capítulo é apresentado o projeto otimizado de coletores piezocompósitos de casca operando em regime harmônico amortecido. Os coletores de energia são estruturas capazes de transformar a energia mecânica (em forma de vibrações) em energia elétrica que possa ser utilizado por um outro dispositivo. Assim, deseja-se maximizar a potência elétrica (medida em um circuito elétrico acoplado) gerada pela estrutura piezocompósita excitado por deslocamentos mecânicos harmônicos em uma região conhecida.

Recentemente, coletores de energia, ou "energy harvesting" como é conhecido na literatura, tem recebido grande atenção na área de transdutores piezelétricos. Neste tipo de transdutores, materiais piezelétricos são geralmente incorporados à estruturas para aproveitar vibrações e deformações provenientes do ambiente em que ela está inserida para transformá-las em energia elétrica aproveitável. Com os avanços nas técnicas de miniaturização e portabilidade de equipamentos eletrônicos, como sensores e atuadores, aumenta-se a necessidade de se reduzir ou eliminar fontes externas de energia elétrica ou baterias. Neste sentido, materiais piezelétricos são muito promissores pois eles podem ser diretamente incorporados à estrutura e a energia gerada pode ser diretamente utilizada (BEEBY; TUDOR; WHITE, 2006; RUPP et al., 2009). Diversos trabalhos têm sido publicados nesse assunto (SODANO; INMAN, 2004; DUTOIT; WARDLE; KIM, 2005; BEEBY; TUDOR; WHITE, 2006; ANTON; SODANO, 2007; PRIYA, 2007; COOK-CHENNAULT; THAMBI; SASTRY, 2008; ERTURK; INMAN, 2008; RUPP et al., 2009; RUPP; DUNN; MAUTE, 2010). Muitas configurações de coletores de energia consistem em estruturas de placas e cascas cobertas por camadas de material piezelétrico em um ou nos dois lados (RUPP et al., 2009). 
Esses tipos de estrutura são geralmente utilizados pois eles permitem uma área maior de geração de energia (quanto maior a área sujeita à deformações mecânicas, maior é a produção de energia). Neste sentido, existe um vasto campo de pesquisa com o objetivo de coletar a energia que seria descartada ao ambiente e transformá-la em energia utilizável, principalmente em dispositivos embarcados de baixo consumo.

Assim como no projeto de atuadores, técnicas de otimização topológica têm sido amplamente utilizadas para se projetar os coletores de energia de modo a aproveitar ao máximo a conversão de energia. Zheng, Chang e Gea (2009) utilizam uma metodologia de otimização topológica para aplicações estáticas de placa piezelétrica com o intuito de maximizar a conversão de energia mecânica em elétrica. Rupp et al. (2009), Rupp, Dunn e Maute (2010) consideraram o projeto dinâmico de coletores de energia e otimizam a topologia e o sentido de polarização das camadas piezelétricas acopladas à uma camada de material isotrópico, com um circuito elétrico externo de coleta de energia acoplado à estrutura. Já Chen et al. (2010) projetaram coletores de energia utilizando um método de otimização topológica baseado no método de "level-sets".

Alguns desses trabalhos sugerem ainda utilizar uma massa concentrada em algum ponto da estrutura para que as amplitudes de vibração sejam ampliadas, produzindo maior potência elétrica. Entretanto, aumentando-se as amplitudes dos deslocamentos, aumenta-se também o valor das tensões mecânicas na estrutura. Materiais piezelétricos são frágeis e, além disso, possuem um limite de tensão que gera despolarização, cujo valor é muito inferior à tensão de ruptura do material. Assim, propõe-se neste trabalho obter topologias ótimas de coletores de energia onde a distribuição de material piezelétrico sobre o substrato consiga gerar quantidade significativa de energia sem utilizar massas concentradas, respeitando os limites de tensão mecânica. Portanto, este trabalho traz uma nova abordagem no projeto de coletores de energia.

Este capítulo está organizado da seguinte maneira: primeiramente é apresentada uma modificação no sistema de equações do MEF para adaptar a problemas de coletores de energia com circuito elétrico acoplado, excitados por deslocamentos harmônicos conhecidos. Na continuação, o problema de otimização topológica para coletores de energia é formulado para o domínio contínuo e para o domínio discretizado. A próxima seção mostra o cálculo da sensibilidades da função objetivo e restrições com relação às variáveis de projeto. Na sequência são apresentados resultados numéricos para exemplificar o método. Finalmente, as conclusões desta parte do trabalho são apresentadas. 


\subsection{Excitação por Deslocamento Mecânico e Modelagem dos Eletrodos}

No projeto de coletores de energia piezocompósitos, a estrutura é excitada pela movimentação de uma base oscilante, como representado na Fig. 6.1.

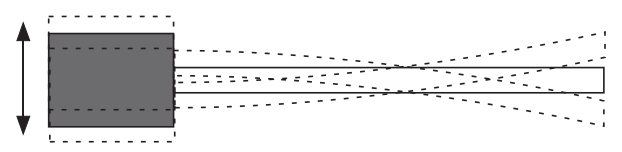

Figura 6.1: Representação de uma estrutura com excitação por movimento de uma base oscilante.

Considera-se então que no sistema de equações de equilíbrio dinâmico amortecido, com resistor acoplado, do MEF descrito pela Eq. (2.46), é necessário prescrever os deslocamentos conhecidos para ser possível calcular os deslocamentos do restante da estrutura e o potencial elétrico gerado nos eletrodos do material piezelétrico. Separando-se os graus de liberdade dos deslocamentos conhecidos (ou prescritos) como $\mathbf{U}_{p}$ e os graus de liberdade dos deslocamentos desconhecidos $\mathbf{U}_{d}$, e considerando o acoplamento dos graus de liberdade elétrico para modelar os eletrodos apresentado pela Eq. (5.3), pode-se reescrever a Eq. (2.46) da seguinte maneira:

$$
\left.\begin{array}{ccccc}
\mathbf{K}_{\mathbf{u}_{p} \mathbf{u}_{p}}-\omega^{2} \mathbf{M}_{p p}+i \omega \mathbf{C}_{p p} & \mathbf{K}_{\mathbf{u}_{p} \mathbf{u}_{d}}-\omega^{2} \mathbf{M}_{p d}+i \omega \mathbf{C}_{p d} & \mathbf{K}_{\mathbf{u}_{p} \phi} & \overline{\mathbf{A}}_{p}^{T} & \mathbf{0} \\
\mathbf{K}_{\mathbf{u}_{p} \mathbf{u}_{d}}^{T}-\omega^{2} \mathbf{M}_{p d}^{T}+i \omega \mathbf{C}_{p d}^{T} & \mathbf{K}_{\mathbf{u}_{d} \mathbf{u}_{d}}-\omega^{2} \mathbf{M}_{d d}+i \omega \mathbf{C}_{d d} & \mathbf{K}_{\mathbf{u}_{d} \phi} & \overline{\mathbf{A}}_{d}^{T} & \mathbf{0} \\
\mathbf{K}_{\mathbf{u}_{p} \phi}^{T} & \mathbf{K}_{\mathbf{u}_{d} \phi}^{T} & \mathbf{K}_{\phi \phi}+i \omega \mathbf{K}_{R} & \mathbf{0} & \mathbf{A}_{E}^{T} \\
\overline{\mathbf{A}}_{p} & \overline{\mathbf{A}}_{d} & \mathbf{0} & \mathbf{0} & \mathbf{0} \\
\mathbf{0} & \mathbf{0} & \mathbf{A}_{E} & \mathbf{0} & \mathbf{0}
\end{array}\right]\left\{\begin{array}{c}
\mathbf{U}_{p} \\
\mathbf{U}_{d} \\
\mathbf{\Phi} \\
\boldsymbol{\lambda}_{1} \\
\boldsymbol{\lambda}_{2}
\end{array}\right\}=
$$

sendo que em $\boldsymbol{\Phi}$ estão inclusos os graus de liberdade elétricos internos e de eletrodo, e as matrizes $\overline{\mathbf{A}}$ e $\mathbf{A}_{E}$ são as matrizes de acoplamento entre camadas e de potencial elétrico, respectivamente, definidas nas seções 2.7 e 5.1. Deve-se salientar que o sistema da Eq. (6.1) gera números complexos como deslocamento, exceto pelos deslocamentos prescritos que possuem apenas valores reais. O sistema da Eq. (6.1) é resolvido utilizando-se o software 
Matlab.

Sabendo-se que $\mathbf{F}_{p}$ é nulo e utilizando $\mathbf{U}_{p}$ como a excitação de base, o sistema da Eq. (6.1) pode ser simplificado como:

$$
\begin{gathered}
{\left[\begin{array}{cccc}
\mathbf{K}_{\mathbf{u}_{d} \mathbf{u}_{d}}-\omega^{2} \mathbf{M}_{d d}+i \omega \mathbf{C}_{d d} & \mathbf{K}_{\mathbf{u}_{d} \phi} & \overline{\mathbf{A}}_{d}^{T} & \mathbf{0} \\
\mathbf{K}_{\mathbf{u}_{d} \phi}^{T} & \mathbf{K}_{\phi \phi}+i \omega \mathbf{K}_{R} & \mathbf{0} & \mathbf{A}_{E}^{T} \\
\overline{\mathbf{A}}_{d} & \mathbf{0} & \mathbf{0} & \mathbf{0} \\
\mathbf{0} & \mathbf{A}_{E} & \mathbf{0} & \mathbf{0}
\end{array}\right]\left\{\begin{array}{c}
\mathbf{U}_{d} \\
\mathbf{\Phi} \\
\boldsymbol{\lambda}_{1} \\
\boldsymbol{\lambda}_{2}
\end{array}\right\}=} \\
=-\left[\begin{array}{c}
\mathbf{K}_{\mathbf{u}_{p} \mathbf{u}_{d}}^{T}-\omega^{2} \mathbf{M}_{p d}^{T}+i \omega \mathbf{C}_{p d}^{T} \\
\mathbf{K}_{\mathbf{u}_{p} \phi}^{T} \\
\overline{\mathbf{A}}_{p} \\
0
\end{array}\right] \mathbf{U}_{p}
\end{gathered}
$$

$\mathrm{Ou}$

$$
\tilde{\mathbf{K}} \tilde{\mathbf{U}}=\tilde{\mathbf{F}}
$$

Deve-se considerar também que os graus de liberdade de potencial elétrico nos elementos $(\Delta \phi)$ de toda uma camada estão acoplados de forma a produzirem apenas um valor de potencial elétrico, ou seja, esse acoplamento representa a utilização de eletrodos nas superfícies dessa camada. Assim, o mesmo procedimento da seção 5.1 deve ser considerado.

\subsection{Formulação do Problema de OT para Coletores de Energia no Domínio Contínuo}

Considere um domínio genérico apresentado na Fig. 6.2. Ao invés de ser aplicada forças como no projeto de sensores, a estrutura é excitada pela excitação de deslocamentos de uma base. Essa base é representada pelo bloco preto na Fig. 6.2 que oscila em apenas uma direção, $z$ por exemplo. Essa oscilação gera um potencial elétrico $\Delta \phi$ entre os eletrodos da camada piezelétrica, os quais estão ligados à uma resistência elétrica $R$ que simula um dispositivo que será alimentado.

A saída do coletor de energia é medida como a potência elétrica gerada na resistência elétrica $R$. A potência pode ser medida pela seguinte expressão (RUPP et al., 2009):

$$
\wp=\frac{|\Delta \phi|^{2}}{2 R}
$$




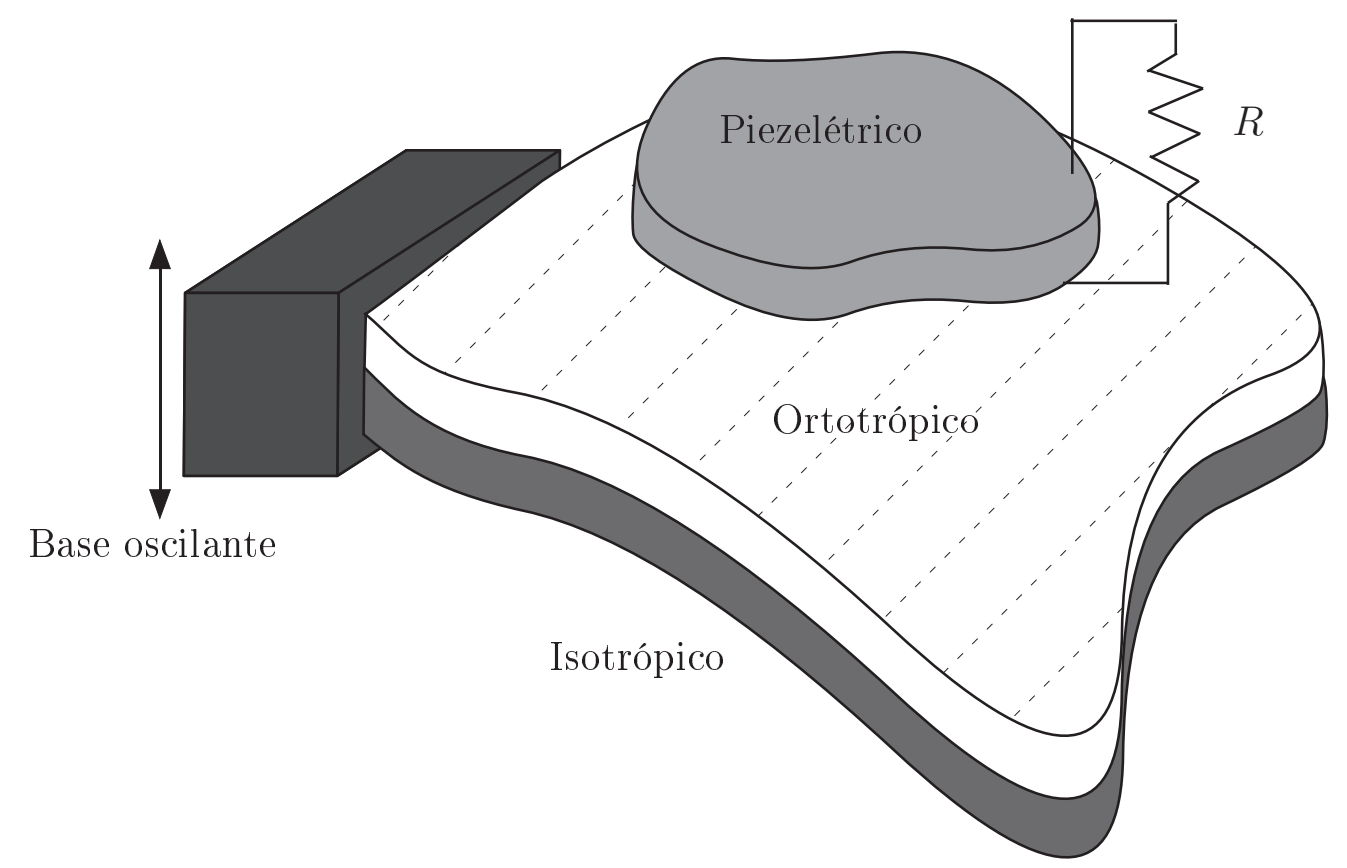

Figura 6.2: Representação do domínio de projeto de um coletor de energia piezocompósito.

o que também pode ser considerada como função objetivo.

O operador módulo é necessário pois $\Delta \phi$ é um número complexo, proveniente da solução do sistema dinâmico amortecido da Eq. (2.46).

Assim, o objetivo da otimização neste projeto é maximizar a potência elétrica medida no resistor $R$, a qual é gerada pela excitação de uma base oscilante na qual a estrutura está engastada, e sujeita a restrição de falha mecânica. A restrição de falha é a mesma utilizada no projeto de sensores, ou seja, deseja-se que o valor mínimo do fator de segurança calculado em toda a estrutura seja maior ou igual a 1, assim como no projeto dos sensores.

Assim como no projeto de atuadores e sensores, no domínio contínuo, a distribuição de material, a polarização do material piezelétrico e a orientação das fibras do material ortotrópico são parametrizadas pelas pseudo-densidades $\gamma$, pelas variáveis de polarização $\varrho$ e pelos ângulos $\boldsymbol{\theta}$, respectivamente. Portanto, o problema de otimização para coletores 
de energia piezocompósitos pode ser formulado, no domínio contínuo, da seguinte maneira:

$$
\begin{array}{cl}
\underset{\boldsymbol{\gamma}, \boldsymbol{\varrho}, \boldsymbol{\theta}}{\operatorname{Maximizar}:} & \wp=\frac{|\Delta \phi|^{2}}{2 R} \\
\text { sujeito à: } & \text { Equações de equilíbrio } \\
& s_{N P}=\left(\sum_{g=1}^{n_{\sigma}} s_{g}^{p_{\sigma}}\right)^{1 / p_{\sigma}} \geq 1 \\
& 0 \leq \boldsymbol{\gamma}(\mathbf{x}) \leq 1 \\
& 0 \leq \boldsymbol{\varrho}(\mathbf{x}) \leq 1 \\
& -90^{\circ} \leq \boldsymbol{\theta}(\mathbf{x}) \leq 90^{\circ}
\end{array}
$$

onde $\Delta \phi$ é o potencial elétrico obtido devido a aplicação de deslocamento de base. Além disso, as equações de equilíbrio estão sujeitas às equações de acoplamento entre camadas, descrita na Seção 2.7. No projeto de coletores de energia, também está sendo adotada uma formulação de otimização topológica sem restrição de volume, como pode ser observado na Eq. (5.5). Assim, é possível obter o volume ótimo de material piezelétrico que deve ser utilizado para maximizar o potencial elétrico gerado pelo material piezelétrico.

\subsection{Formulação do Problema de OT para Coletores de Energia no Domínio Discretizado}

Para implementar o MOT de acordo com o fluxograma apresentado na Seção 3.4 é necessário transformar o problema de otimização, apresentado anteriormente, do domínio contínuo para o discretizado. Esta seção apresenta a função objetivo e a formulação do problema de otimização no domínio discretizado.

Primeiramente, apresenta-se a função $\wp$ escrita no domínio discretizado da seguinte maneira:

$$
\wp=\frac{\left|\mathbf{F}_{\text {aux }}^{T} \tilde{\mathbf{U}}\right|^{2}}{2 R}
$$

onde $\mathbf{F}_{\text {aux }}$ (que tem o mesmo tamanho de $\tilde{\mathbf{U}}$ ) é o vetor global de força auxiliar que contém valor igual a 1 somente no grau de liberdade de potencial elétrico do elemento ao qual está conectado o resistor $R$ e valor nulo nos graus de liberdade restantes, e $\tilde{\mathbf{U}}$ é o vetor global de deslocamentos mecânicos e potenciais elétricos, obtido a partir da solução do sistema linear dinâmico amortecido da Eq. (5.3).

No domínio discretizado, a distribuição de material e a orientação das fibras são discretizadas pelas variáveis topológicas $\mathbf{d}$ de acordo com a técnica de projeção, e pelas 
variáveis de orientação $\vartheta$ de acordo com a OMD, respectivamente. A polarização continua sendo representada pela variável de polarização $\varrho$. Assim, o problema de otimização para coletores de energia piezocompósitos pode ser formulado, no domínio discretizado, da seguinte maneira:

$$
\begin{array}{ll}
\underset{\mathbf{d}, \boldsymbol{\varrho}, \boldsymbol{\vartheta}}{\operatorname{aaximizar}:} & \wp=\frac{\left|\mathbf{F}_{u u x}^{T} \tilde{\mathbf{U}}\right|^{2}}{2 R} \\
\text { sujeito à: } & \tilde{\mathbf{K}} \tilde{\mathbf{U}}=\tilde{\mathbf{F}} \\
& s_{N P}=\left(\sum_{g=1}^{n_{\sigma}} s_{g}^{p_{\sigma}}\right)^{1 / p_{\sigma}} \geq 1 \\
& 0 \leq \mathbf{d} \leq 1 \\
& 0 \leq \boldsymbol{\varrho} \leq 1 \\
& 0 \leq \boldsymbol{\vartheta} \leq 1
\end{array}
$$

lembrando que o valor de $p_{\sigma}$ deve ser negativo para que $s_{N P}$ se aproxime do valor mínimo do fator de segurança calculado na estrutura, como descrito na seção 3.3.3.

\subsection{Análise de Sensibilidades}

Como os deslocamentos são números complexos, a sua derivada deve considerar a parte real e a parte imaginária. Assim, derivando-se a Eq. (6.6), tem-se:

$$
\frac{\partial \wp}{\partial v}=\frac{\left|\mathbf{F}_{\text {aux }}^{T} \tilde{\mathbf{U}}\right|}{R} \frac{\partial\left|\mathbf{F}_{\text {aux }}^{T} \tilde{\mathbf{U}}\right|}{\partial v}
$$

onde a variável $v$ é novamente utilizada para simplificar o desenvolvimento das derivadas, sendo que, posteriormente, ela deve ser substituída por $d$, $\varrho$ e $\vartheta$.

Considerando que $\mathbf{F}_{\text {aux }}^{T} \tilde{\mathbf{U}}$ é um número complexo na forma $a+b i$, e que o módulo desse número complexo pode ser calculado como:

$$
|a+b i|=\sqrt{a^{2}+b^{2}}
$$

então, a derivada de $\mathbf{F}_{\text {aux }}^{T} \tilde{\mathbf{U}}$ pode ser calculada da seguinte maneira:

$$
\begin{array}{r}
\frac{\partial\left|\mathbf{F}_{a u x}^{T} \tilde{\mathbf{U}}\right|}{\partial v}=\frac{1}{\left|\mathbf{F}_{a u x}^{T} \tilde{\mathbf{U}}\right|}\left\{\Re\left[\mathbf{F}_{\text {aux }}^{T} \tilde{\mathbf{U}}\right] \Re\left[\frac{\partial\left(\mathbf{F}_{a u x}^{T} \tilde{\mathbf{U}}\right)}{\partial v}\right]+\right. \\
\left.+\Im\left[\mathbf{F}_{\text {aux }}^{T} \tilde{\mathbf{U}}\right] \Im\left[\frac{\partial\left(\mathbf{F}_{a u x}^{T} \tilde{\mathbf{U}}\right)}{\partial v}\right]\right\}
\end{array}
$$


Substituindo a Eq. (6.10) na Eq. (6.8), e considerando que $\mathbf{F}_{\text {aux }}$ não depende de nenhuma variável de projeto, ou seja, $\partial \mathbf{F}_{\text {aux }}^{T} / \partial v=\mathbf{0}$, tem-se que:

$$
\frac{\partial \wp}{\partial v}=\frac{1}{R}\left\{\Re\left[\mathbf{F}_{\text {aux }}^{T} \tilde{\mathbf{U}}\right] \Re\left[\mathbf{F}_{\text {aux }}^{T} \frac{\partial \tilde{\mathbf{U}}}{\partial v}\right]+\Im\left[\mathbf{F}_{\text {aux }}^{T} \tilde{\mathbf{U}}\right] \Im\left[\mathbf{F}_{\text {aux }}^{T} \frac{\partial \tilde{\mathbf{U}}}{\partial v}\right]\right\}
$$

A derivada $\frac{\partial \tilde{\mathbf{U}}}{\partial v}$ é obtida derivando-se a Eq. (6.2), que resulta em

$$
\frac{\partial \tilde{\mathbf{U}}}{\partial v}=-\tilde{\mathbf{K}}^{-1}\left[\begin{array}{ccc}
\frac{\partial\left(\mathbf{K}_{\mathbf{u}_{p} \mathbf{u}_{d}}^{T}-\omega^{2} \mathbf{M}_{p d}^{T}+i \omega \mathbf{C}_{p d}^{T}\right)}{\partial v} & \frac{\partial\left(\mathbf{K}_{\left.\mathbf{u}_{d} \mathbf{u}_{d}-\omega^{2} \mathbf{M}_{d d}+i \omega \mathbf{C}_{d d}\right)}^{\partial v}\right.}{\frac{\partial \mathbf{K}_{\mathbf{u}_{d} \phi}}{\partial v}} \\
\frac{\partial \mathbf{K}_{\mathbf{u}_{p} \phi}^{T}}{\partial v} & \frac{\partial \mathbf{K}_{\mathbf{u}_{d} \phi}^{T}}{\partial v} & \frac{\partial \mathbf{K}_{\phi \phi}}{\partial v} \\
\mathbf{0} & \mathbf{0} & \mathbf{0}
\end{array}\right]\left\{\begin{array}{c}
\mathbf{U}_{p} \\
\mathbf{U}_{d} \\
\boldsymbol{\Phi}
\end{array}\right\}
$$

lembrando que $\mathbf{K}_{R}$ é constante e sua derivada em relação a qualquer variável é nula.

Substituindo a Eq. (6.12) na Eq. (6.11), é possível aplicar o método adjunto para evitar muitas inversões de matrizes. Ou seja, a Eq. (6.11) pode ser reescrita da seguinte maneira:

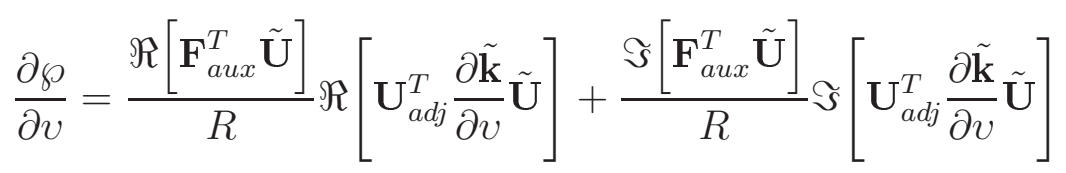

onde

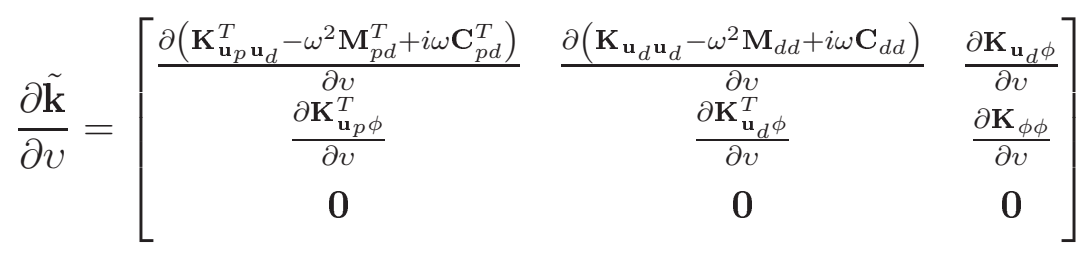

e $\mathbf{U}_{a d j}$ é a solução de um sistema linear na forma:

$$
\tilde{\mathbf{K}} \mathbf{U}_{a d j}=\mathbf{F}_{a u x}
$$

A derivada da restrição de falha é apresentada na seção 3.5.

\subsection{Resultados}

Nesta seção, são apresentados resultados numéricos de dois exemplos para o projeto otimizado de coletores de energia piezocompósitos de casca. O primeiro exemplo utiliza uma estrutura plana que representa uma placa quadrada e o segundo exemplo utiliza uma estrutura curva que representa um quarto de cilindro. Para cada exemplo, são estudados dois casos de otimização para demonstrar a metodologia proposta aplicada a projeto de 
coletores de energia:

caso 1: substrato de alumínio coberto por uma camada de material piezelétrico, considerando otimização de distribuição e de polarização do material piezelétrico;

caso 2: substrato composto de uma camada de fibra de carbono coberto por uma camada de alumínio, e mais uma camada de material piezelétrico sobre elas, considerando otimização de distribuição e de polarização do material piezelétrico e otimização de orientação das fibras.

Em ambos os casos é utilizada a restrição de falha mecânica proposta neste trabalho. As propriedades dos materiais utilizados podem ser encontrados no Apêndice C sendo que neste trabalho utiliza-se o material piezelétrico da Tab. C.3.

As otimizações de distribuição de material e de sentido de polarização são aplicadas somente às camadas de material piezelétrico para verificar sua influência no valor da potência elétrica gerada. As camadas de substrato não sofrem alteração da topologia, somente de orientação de fibras no caso da fibra de carbono. Essa abordagem é adotada para evitar a utilização de outra função objetivo para minimizar a flexibilidade da estrutura, como a função de flexibilidade média (SILVA et al., 1999; SILVA; NISHIWAKI; KIKUCHI, 2000; CARBONARI; SILVA; NISHIWAKI, 2005, 2007). Esse tipo de função funciona, basicamente, minimizando os deslocamentos na estrutura, o que prejudicaria a eficácia da função de maximização da potência elétrica gerada.

Para a otimização de orientação dos ângulos de fibras no caso 2, são adotados 12

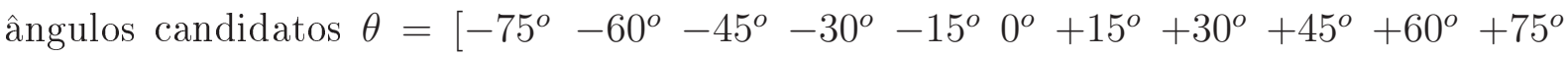
$\left.+90^{\circ}\right]$. Neste trabalho, atribui-se, inicialmente, valores iguais para todas as variáveis de topologia $d=0,5$ e de polarização $\varrho=0,6$. Não se utiliza o valor $\varrho=0,5$ para não gerar polarização nula logo no início do processo de otimização e criar instabilidades numéricas no método. O valor inicial para as variáveis de orientação deve ser igual a 0,5 de modo que nenhum ângulo candidato seja favorecido no início do processo de otimização. Para todos os exemplos apresentados, foram realizadas várias análises utilizando diferentes valores para as variáveis iniciais, que no final de cada análise, resultou em praticamente os mesmos resultados com valores de deslocamento muito próximos, com diferenças no valor da potência gerada de no máximo $2 \%$. Assim, é possível afirmar que para os exemplos apresentados, chegou-se a soluções ótimas que estão muito próximas ao ótimo global.

Nas figuras de distribuição de material, são apresentados os valores das pseudo-densidades, onde a cor preta representa o elemento cheio e a cor branca representa 
o elemento vazio. Valores intermediários das pseudodensidades são apresentadas em escalas de cinza. Nas figuras de polarização, os elementos em preto e branco possuem polarização positiva e negativa, respectivamente. Nas figuras de otimização de orientação, são apresentadas as orientações ótimas em cada elemento por meio de uma reta. Nas figuras das deformadas, as camadas em verde e azul representam as camadas piezelétricas com polarização negativa e positiva, respectivamente, e as camadas em vermelho e roxo representam as camadas de alumínio e de fibra de carbono, respectivamente.

Finalmente, os valores dos coeficientes de penalização do modelo PEMAP-P são baseados nos trabalhos de Kögl e Silva (2005). Assim, $p_{\epsilon}=p_{i}=1, p_{c}=3$ e $p_{e}=4$. Entretanto, Kögl e Silva (2005) faz um estudo de projetos estáticos e não inclui a matriz de massa e nem o coeficiente $p_{m}$. Foram feitos alguns testes com os exemplos apresentados e chegou-se a um valor de $p_{m}=3$ que minimiza o aparecimento das pseudodensidades intermediárias. Para evitar que a solução fique "presa" num mínimo local por causa dos expoentes $p_{c}$ e $p_{e}$, utiliza-se o método da continuação nesses valores. Inicialmente, $p_{c}=p_{e}=p_{m}=1$. Nas iterações 10, 20 e 30, $p_{e}$ e $p_{m}$ são incrementados em uma unidade, e nas iterações 30 e 40, $p_{c}$ é incrementado em uma unidade. Para o coeficiente de penalização da orientação $p_{\vartheta}$ também é utilizada a técnica da continuação como proposto por Stegmann e Lund (2005a). Neste trabalho, inicialmente $p_{\vartheta}=1$, e tem seu valor aumentado em uma unidade nas iterações 10, 20, 30, 40 e 50, finalizando com valor igual a 6. Nos casos estudados aqui, é utilizado amortecimento estrutural onde $\beta_{D}=10^{-5}$ para estabilizar a amplitude de vibração se a estrutura entrar em ressonância.

\subsection{Exemplo 1}

Neste exemplo, é utilizado um domínio de placa quadrada, conforme apresentado na Fig. 6.3, de lados iguais a $l=100 \mathrm{~mm}$. O raio utilizado na técnica de projeção é igual a 3,5 mm. Os ângulos das orientações das fibras são também representadas na Fig. 4.18 onde o ângulo $0^{\circ}$ segue o eixo y e $90^{\circ}$ segue o eixo $x$. O sentido de polarização do material piezelétrico segue o sentido da direção $z$. As espessuras das camadas de substrato e de material piezelétrico são iguais a $t_{s}=0,4 \mathrm{~mm}$ e $t_{p}=0,2 \mathrm{~mm}$, respectivamente.

A estrutura está engastada no bloco cinza escuro, como pode ser visto na Fig. 6.3, sendo que a dimensão a é igual a $30 \mathrm{~mm}$. Entretanto, somente a camada de substrato está engastada, ou seja, a camada de material piezelétrico está apenas colada sobre o substrato e não é presa no engaste. Essa abordagem aproxima a modelagem de uma estrutura real, já que a fixação geralmente é feita apenas no substrato. A excitação de 


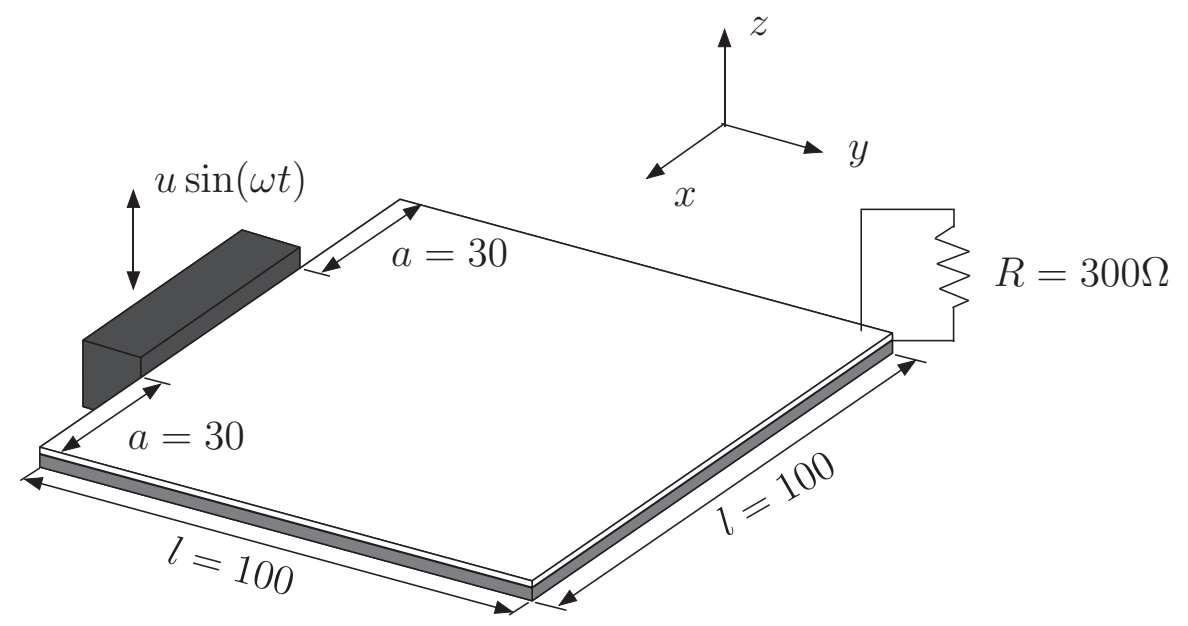

Figura 6.3: Domínio de projeto para coletores de energia de placa. Medidas em mm.

base tem amplitude igual a $u=1 \mathrm{~mm}$ na direção de $z$, e a frequência de excitação é igual a $f_{\omega}=200 \mathrm{~Hz}$ pois está próxima à uma frequência de ressonância, frequência na qual são gerados os maiores valores de potência elétrica. A frequência angular $\omega$ é igual a $\omega=2 \pi f_{\omega}$. A resistência elétrica utilizada tem valor igual a $R=300 \Omega$.

Uma análise de convergência da malha de elementos finitos revelou que a convergência nas análises, tanto de potência elétrica, quanto de tensões mecânicas, é obtida para uma discretização de 30x30 elementos. Não foi utilizada uma malha mais refinada pois o elemento de casca multicamada utilizado, junto com o método OMD são muito custosos computacionalmente.

A Fig. 6.4 apresenta a forma deformada da estrutura sem aplicar nenhuma otimização, ou seja, a camada de material piezelétrico está totalmente cheia e com polarização positiva. Contudo, como a potência é uma função módulo, o sentido de polarização não faz diferença. Esse caso será chamado de caso 0, e obteve-se uma potência elétrica gerada igual a 0,023 W. Esse baixo valor de potência elétrica é devido a toda camada ter o mesmo sentido de polarização, sendo que, enquanto uma região gera um potencial positivo, uma outra região gera potencial negativo. Como os graus de liberdade elétricos estão acoplados, a somatória final acaba gerando baixas potências.

Além disso, analisando a distribuição de tensões da Fig. 6.5, nota-se que existem regiões nas duas camadas que apresentam tensões acima do limite do material. Na camada de alumínio, o valor máximo de von Mises é igual a 876,96 MPa e na camada de material piezelétrico é igual a $537,38 \mathrm{MPa}$, ou seja, estão muito acima dos limites de escoamento do alumínio e de despolarização do material piezelétrico, respectivamente. Os altos valores de tensão são decorrentes do alto valor da amplitude de oscilação da estrutura, que nesse 


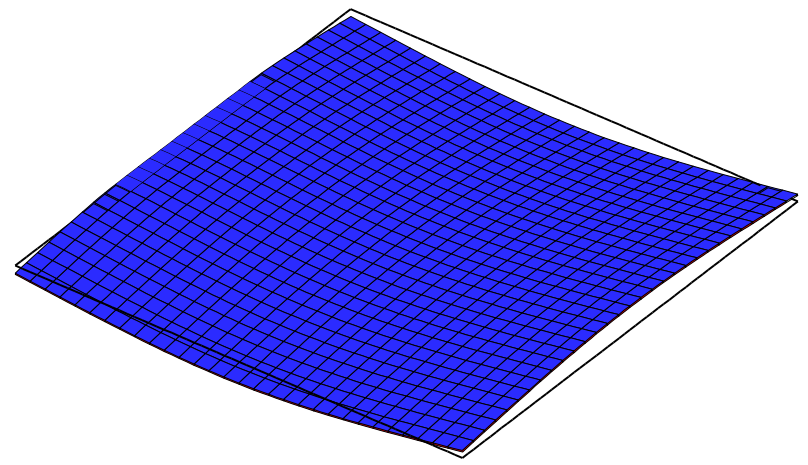

Figura 6.4: Forma deformada da estrutura para o caso 0 de coletores de energia de placa, ou seja, sem otimização, com a camada piezelétrica totalmente cheia e polarização positiva.

caso chega a ser igual a 7,36 mm, devido ao fator dinâmico da quantidade de material utilizada. Assim, a restrição de falha mecânica torna-se necessária no projeto de coletores de energia.

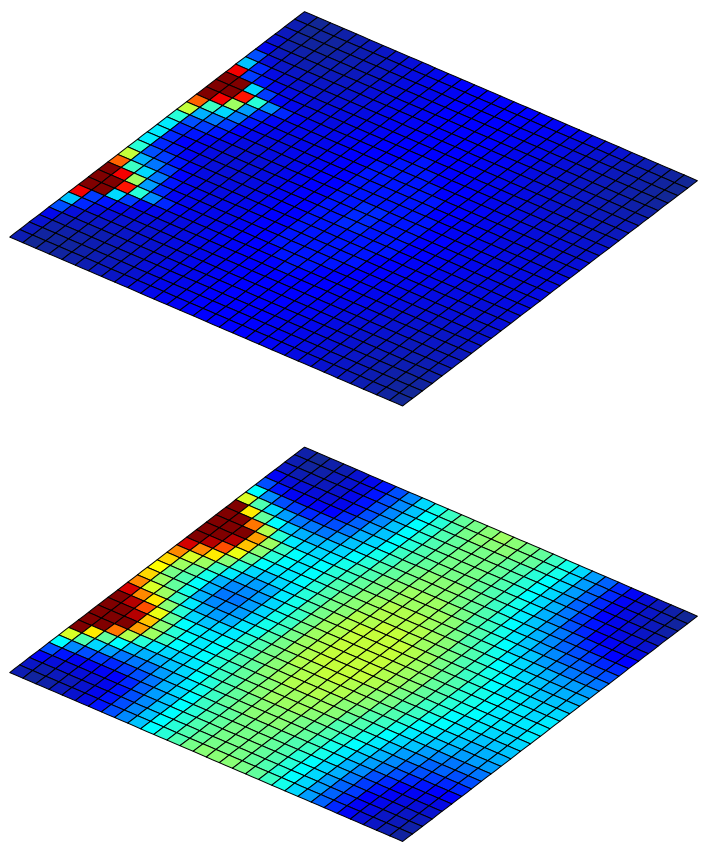

(a) Camada de alumínio

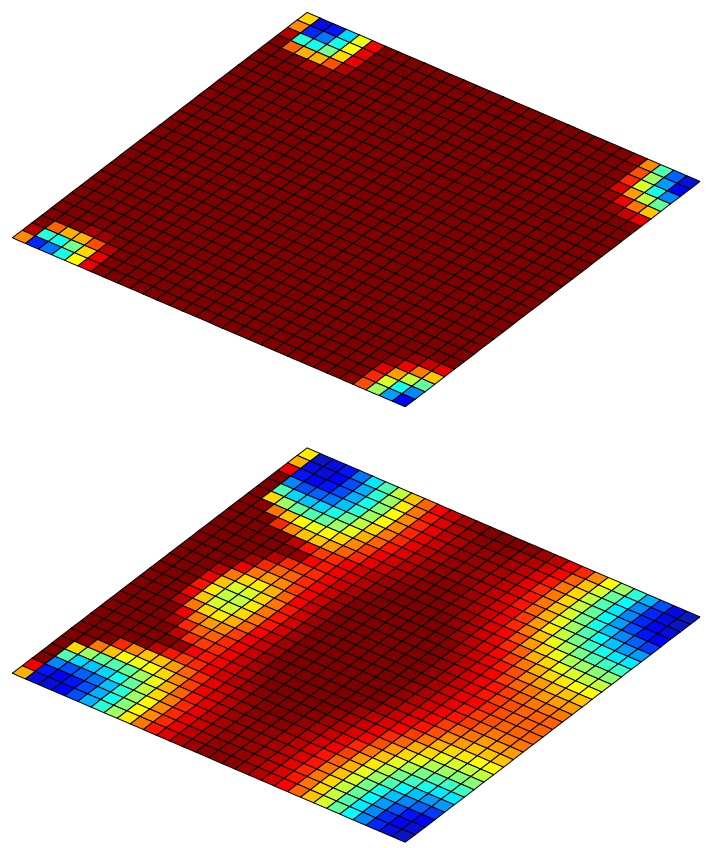

(b) Camada piezelétrica

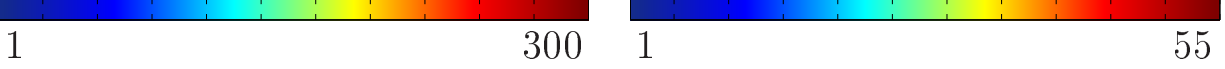

Figura 6.5: Distribuição das tensões de von Mises das superfícies superior e inferior das camadas de material piezelétrico para o caso 0 de coletores de energia de placa. Valores de tensão em MPa, onde os valores máximos da escala representam os limites de tensão.

As Figs. 6.6(a) e 6.6(b) referem-se aos resultados de otimização de topologia e de polarização para o caso 1 e a Fig. 6.7 apresenta sua respectiva deformada pós-processada. 
O valor da potência elétrica obtida neste caso é igual a $0,13 \mathrm{~W}(0,129 \mathrm{~W}$ para o resultado

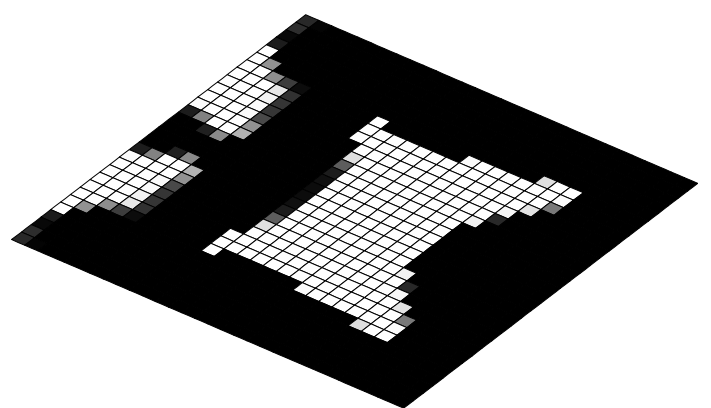

(a) Resultado de otimização topológica

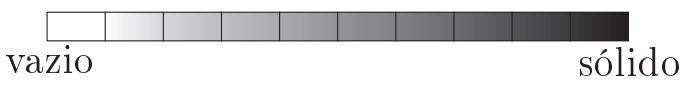

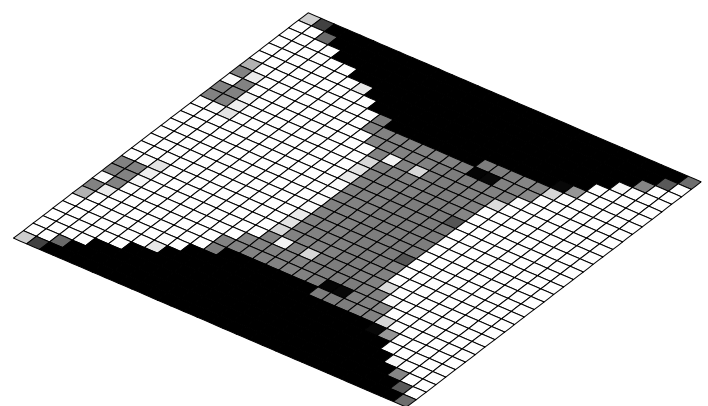

(b) Resultado de otimização de polarização

Figura 6.6: Resultado da otimização de topologia (a) e de polarização (b) das camadas piezelétricas para o caso 1 de coletores de energia de placa.

pós-processado), 5,65 vezes maior que no caso 0 . O volume final da camada piezelétrica é igual a 64,88\% do volume total da camada. Nota-se na Fig. 6.6(a) que existem algumas regiões de material intermediário (cinza). Embora a técnica de projeção com a função "degrau" seja eficiente em problemas estáticos, no problema dinâmico alguns outros cuidados devem ser tomados, como por exemplo, a escolha do coeficiente de penalização $p_{m}$. Neste trabalho, o valor utilizado de $p_{m}=3$ conseguiu minimizar essas regiões. Na Fig. 6.6(b) observa-se uma grande região de polarização intermediária (cinza), mas que coincide com as regiões onde não há material piezelétrico, podendo assim, ser desconsiderada.

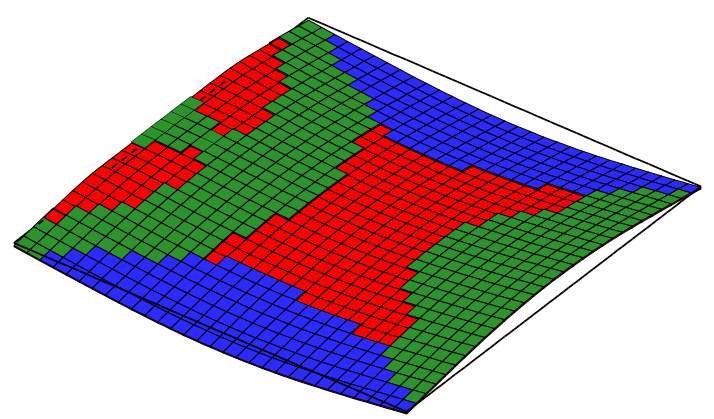

Figura 6.7: Forma deformada do resultado pós-processado para o caso 1 de coletores de energia de placa. Em vermelho está representada a camada de alumínio, e em azul e verde está representada a camada piezelétrica com polarização positiva e negativa, respectivamente.

Na Fig. 6.8 são apresentadas as distribuições de tensão mecânica nas superfícies 
inferior e superior das duas camadas. As tensões máximas de von Mises nas camadas de alumínio e de material piezelétrico são iguais a 212,55 e 45,9 MPa, respectivamente, os quais obedecem aos limites de tensão adotados no problema.

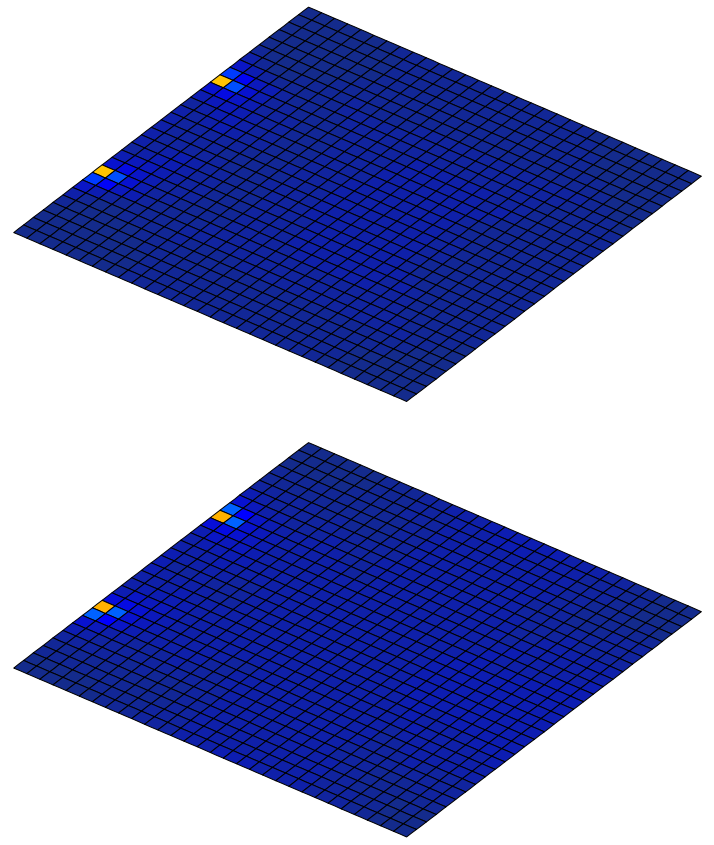

(a) Camada de alumínio

1

300

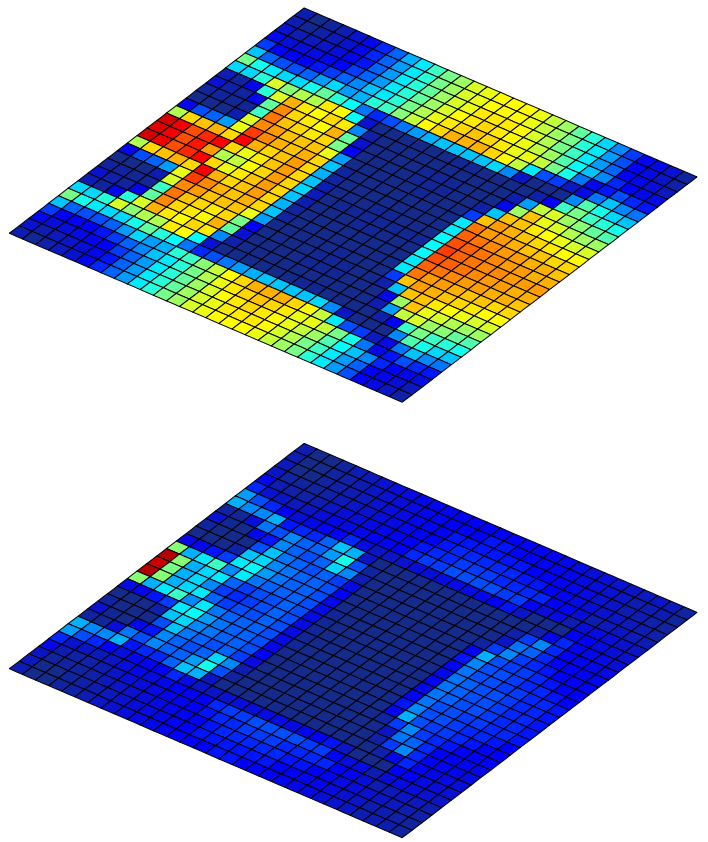

(b) Camada piezelétrica

1
50

Figura 6.8: Distribuição das tensões de von Mises das superfícies superior e inferior das camadas de material piezelétrico para o caso 1 de coletores de energia de placa. Valores de tensão em MPa, onde os valores máximos da escala representam os limites de tensão.

Analisando a topologia na Fig. 6.6(a) e a distribuição de tensões da Fig. 6.8, observa-se que a OT distribuiu material próximo aos contornos da estrutura, porém tentando evitar a região de engaste. Além disso, reduzindo-se a quantidade de material na região central da estrutura, reduz-se também a massa da estrutura e, consequentemente, a amplitude de deslocamentos, que passa a ser menor que $2 \mathrm{~mm}$. Isso foi o fator determinante para a redução nos valores de tensão para que os limites fossem obedecidos. Apesar das amplitudes serem reduzidas, a otimização do sentido de polarização foi responsável pelo aumento no valor da potência elétrica gerada, já que foi possível ajustar adequadamente o sentido de polarização conforme as regiões de tração e de compressão.

Agora para o caso 2, as Figs. 6.9(a), 6.9(b) e 6.10(a) referem-se aos resultados de otimização de topologia, de polarização e de orientação e a Fig. 6.10(b) apresenta sua respectiva deformada do resultado pós-processado. O valor da potência elétrica obtida 


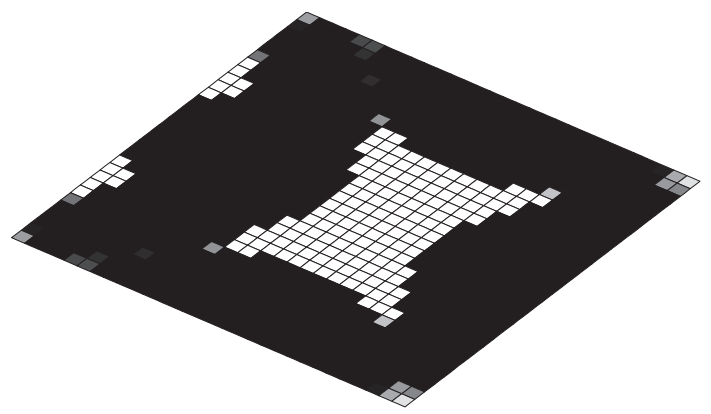

(a) Resultado de otimização topológica

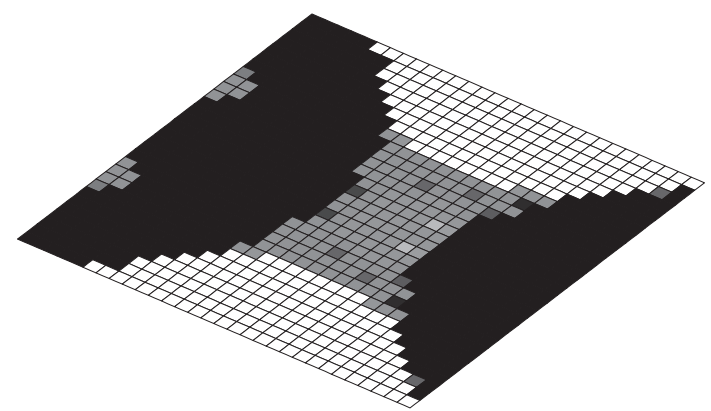

(b) Resultado de otimização de polarização

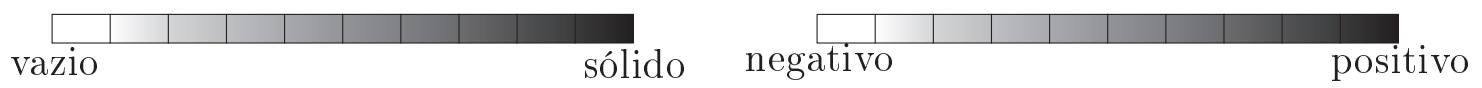

Figura 6.9: Resultado da otimização de topologia (a) e de polarização (b) das camadas piezelétricas para o caso 2 de coletores de energia de placa.

neste caso é igual a $0,21 \mathrm{~W}(0,196 \mathrm{~W}$ para o resultado pós-processado), 1,61 vezes maior que no caso 1 e 9,13 vezes maior que no caso 0 . O volume final da camada piezelétrica é igual a 81,10\% do volume total da camada. Nota-se na Fig. 6.9(a) que existem poucas regiões de material intermediário (cinza) comprovando a eficácia da técnica de projeção com a escolha adequada dos coeficientes de penalização do modelo PEMAP-P. Na Fig. 6.9(b) observa-se uma grande região de polarização intermediária (cinza), mas que também coincide com as regiões onde não há material piezelétrico, podendo assim, ser desconsiderada.
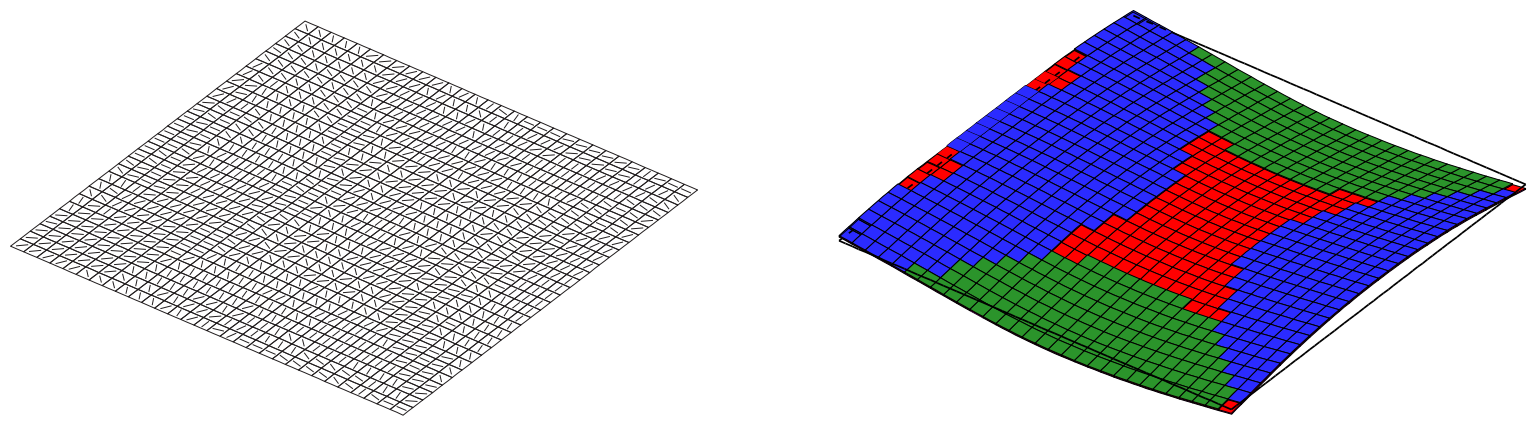

Figura 6.10: Resultado da otimização de orientação de fibras (a) e forma deformada (b) do resultado pós-processado para o caso 2 de coletores de energia de placa. Em vermelho está representada a camada de alumínio, e em azul e verde está representada a camada piezelétrica com polarização positiva e negativa, respectivamente.

Na Fig. 6.11 são apresentadas as distribuições de tensão mecânica nas superfícies inferior e superior das três camadas. A tensão máxima de Tsai-Wu na camada de fibra de carbono é igual a 0,31, e as tensões máximas de von Mises nas camadas de alumínio e de 
material piezelétrico são iguais a 74,63 e 48,08 MPa, respectivamente, os quais obedecem aos limites de tensão adotados no problema.

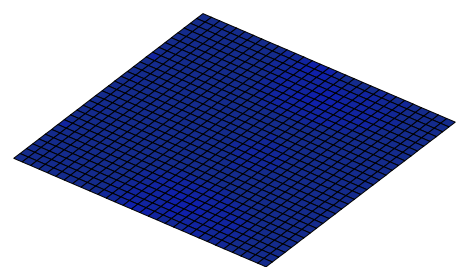

(a) Camada de fibra de carbono - Superfície superior

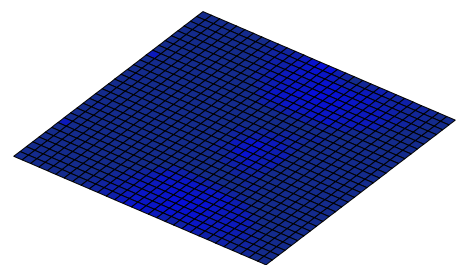

(d) Camada de fibra de carbono - Superfície inferior

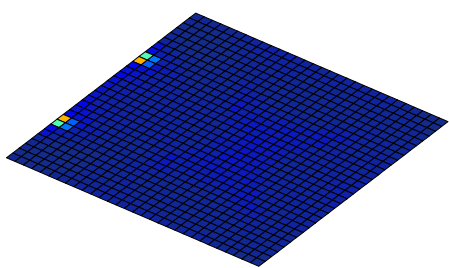

(b) Camada de alumínio Superfície superior

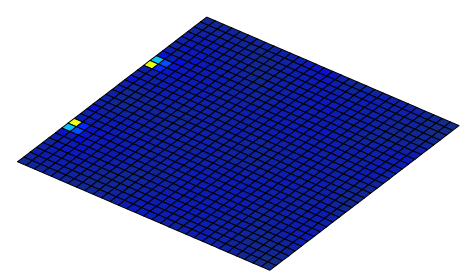

(e) Camada de alumínio Superfície inferior

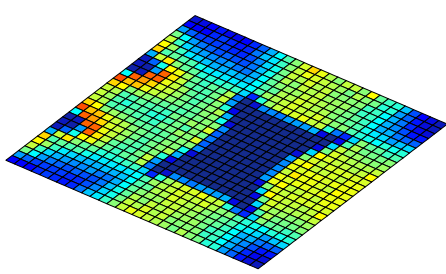

(c) Camada piezelétrica Superfície superior

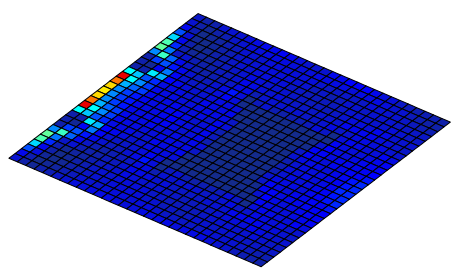

(f) Camada piezelétrica Superfície inferior
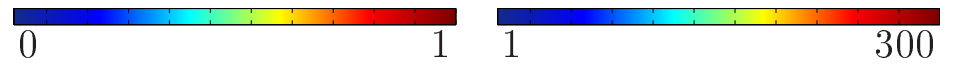

1

50

Figura 6.11: Distribuição das tensões de Tsai-Wu da camada de fibra de carbono e de von Mises das camadas de alumínio e de material piezelétrico para o caso 2 de coletores de energia de placa. Valores de tensão de von Mises em MPa.

Na Fig. 6.11, as imagens de cima e de baixo representam as superfícies superior e inferior, respectivamente, onde os valores máximos da escala representam os limites de tensão para cada critério.

Analisando a topologia na Fig. 6.9(a) e a distribuição de tensões da Fig. 6.11, observa-se que a OT neste segundo caso também distribuiu material próximo aos contornos da estrutura, como no caso 1, também tentando evitar a região de engaste. Pode-se notar agora que foi distribuído mais material do que no caso 1 (15\% a mais), permitindo gerar mais potência pelo fato de ter mais material (e também pela otimização do sentido de polarização, como comentado anteriormente). Todavia, essa quantidade de material a mais foi possível por causa da adequada orientação das fibras na camada de fibra de carbono, o qual, junto com a ausência de material piezelétrico no centro da estrutura, foram responsáveis pela redução dos valores de tensão em todas as camadas, já que o deslocamento máximo da estrutura é igual a $1,84 \mathrm{~mm}$, menos do que no caso 1. 
A Tab. 6.1 apresenta os valores de potência elétrica gerada $(\wp)$, de tensão máxima de von Mises na camada piezelétrica $\left(\sigma_{v m_{\max }}^{\text {piezo }}\right)$ e na camada de alumínio $\left(\sigma_{v m_{\max }}^{A l}\right)$, de tensão máxima de Tsai-Wu na camada de fibra de carbono $\left(\sigma_{t w_{\text {max }}}^{F C}\right)$ e de volume final da camada piezelétrica otimizada $\left(V_{o t}\right)$ para todos os casos apresentados.

Tabela 6.1: Dados dos resultados apresentados para o exemplo de coletores de energia de casca.

\begin{tabular}{|c|c|c|c|c|c|}
\hline & $\begin{array}{l}\wp \\
W\end{array}$ & $\begin{array}{c}\sigma_{v m_{\max }}^{\text {piezo }} \\
\mathrm{MPa}\end{array}$ & $\begin{array}{c}\sigma_{v m_{\max }}^{A l} \\
\mathrm{MPa}\end{array}$ & $\sigma_{t w_{\max }}^{F C}$ & $\begin{array}{l}V_{o t} \\
\%\end{array}$ \\
\hline caso 0 & 0,023 & 537,38 & 876,96 & - & - \\
\hline caso 1 & 0,13 & 45,9 & 212,55 & - & 64,88 \\
\hline caso 2 & 0,21 & 48,08 & 74,63 & 0,16 & 81,10 \\
\hline
\end{tabular}

O gráfico da Fig. 6.12 apresenta a resposta em frequência do coletor de energia. Analisando a linha azul que representa o caso 0, existe uma frequência de ressonância, cujo valor é igual a $190 \mathrm{~Hz}$, que está bem próxima à frequência de excitação de $200 \mathrm{~Hz}$. Isso fez com que a potência gerada tivesse um alto valor. Entretanto, as tensões nesse caso ultrapassavam todos os limites de tensão estabelecidos no problema, como apresentado anteriormente.

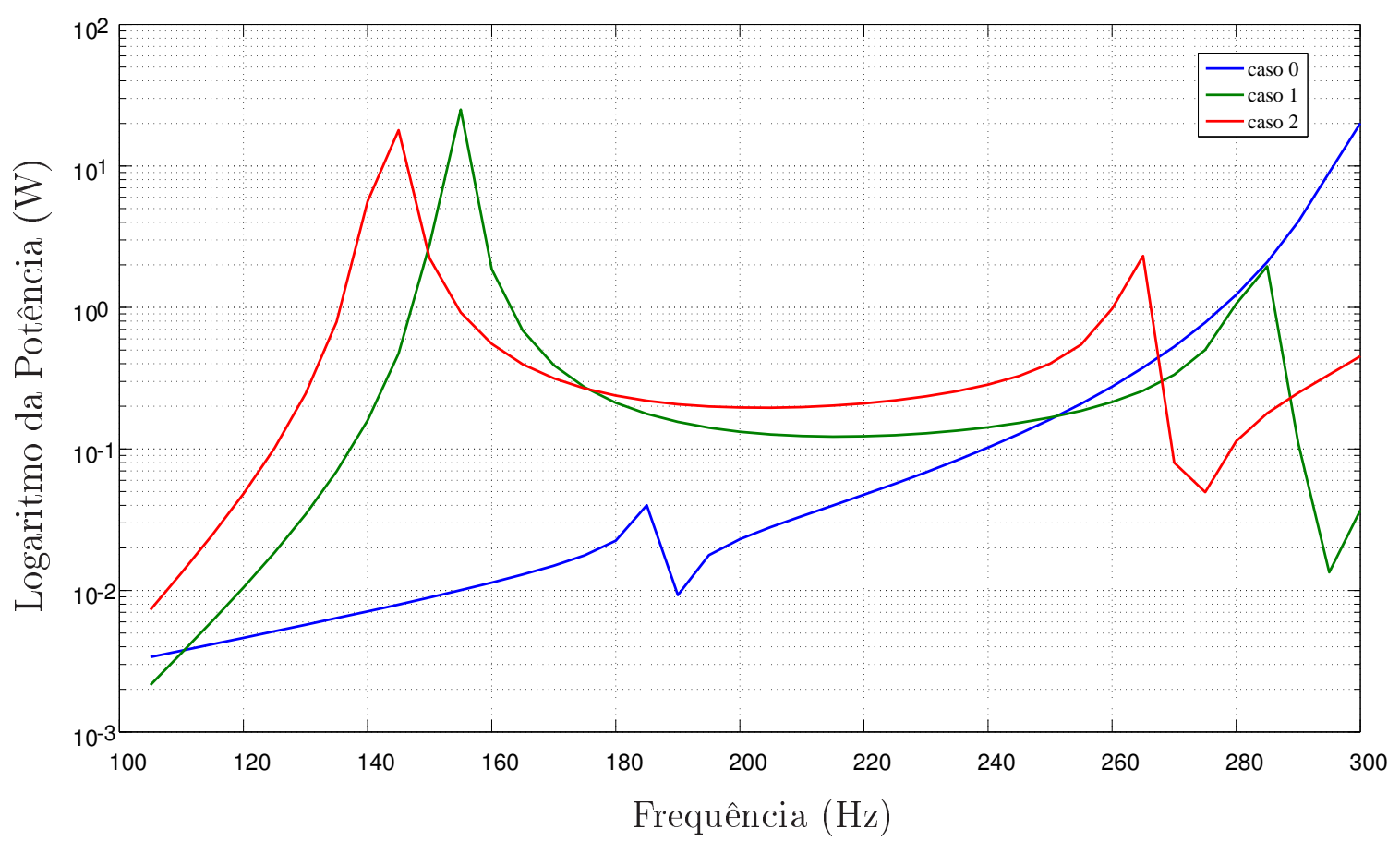

Figura 6.12: Gráfico logarítmico da resposta em frequência dos três casos apresentados anteriormente.

Nos casos 1 e 2, pode-se notar que a estrutura foi ajustada para que as frequências de ressonância não ficassem próximas à frequência de excitação. Esse fenômeno pode ser 
justificado pela utilização da restrição de falha mecânica, que impede que as amplitudes de deslocamento tenham valores muito altos.

No caso 1, representado pela linha verde, observa-se duas frequências de ressonância, uma igual a $170 \mathrm{~Hz}$ e outra igual a $285 \mathrm{~Hz}$. No caso 2, representado pela linha vermelha, tem-se uma resposta bem parecida com a do caso 1, sendo que as frequências são um pouco inferiores, 145 e $265 \mathrm{~Hz}$. Pode-se observar que, em ambos os casos, existe uma região de estabilidade onde os valores de potência elétrica não são tão afetados pela mudança na frequência de operação. Essa região pode ser chamada de faixa de operação, que é uma faixa de frequência na qual o coletor de energia pode trabalhar gerando praticamente o mesmo valor de potência. Entretanto, pode-se verificar que a faixa de operação do caso 1 está em torno de $220 \mathrm{~Hz}$, já no caso 2, essa faixa fica em torno de $200 \mathrm{~Hz}$, ou seja, o resultado do caso 2 apresenta melhor estabilidade na faixa de trabalho. Assim, pode-se concluir que, no caso 2, a otimização simultânea da distribuição e da polarização de material piezelétrico e da orientação das fibras do material ortotrópico, além de gerar maiores valores de potência elétrica na frequência de $200 \mathrm{~Hz}$, foi possível ajustar a faixa de operação para valores mais próximos da frequência de excitação.

\subsection{Exemplo 2}

Neste exemplo, é utilizado um domínio de curvo representando uma estrutura de meio cilindro, conforme apresentado na Fig. 6.13. As dimensões são apresentadas na própria figura e o raio utilizado na técnica de projeção é igual a $5 \mathrm{~mm}$. Os ângulos das orientações das fibras são também representadas na Fig. 4.18 onde o ângulo $0^{\circ}$ segue a direção circunferencial e $90^{\circ}$ segue o eixo z. O sentido de polarização do material piezelétrico segue a direção radial, sendo que a polarização positiva segue o sentido de dentro para fora do cilindro. No caso 2, o substrato é dividido em duas camadas, uma de fibra de carbono e uma de alumínio e suas espessuras são iguais a $t_{c}=0,35 \mathrm{~mm}$ e $t_{a}=0,05$ $\mathrm{mm}$, respectivamente. Nesse caso, a camada de alumínio serve como eletrodo para as camadas piezelétricas, já que a camada de fibra de carbono não é condutora de eletricidade. As propriedades dos materiais utilizados podem ser encontrados no Apêndice C, sendo que o material piezelétrico utilizado é o da Tab. C.3.

A estrutura está engastada no bloco cinza escuro, como pode ser visto na Fig. 6.13. Entretanto, somente a camada de substrato está engastada, ou seja, a camada de material piezelétrico está apenas colada sobre o substrato e não é presa no engaste. Essa abordagem aproxima a modelagem de uma estrutura real, já que a fixação geralmente é feita apenas 


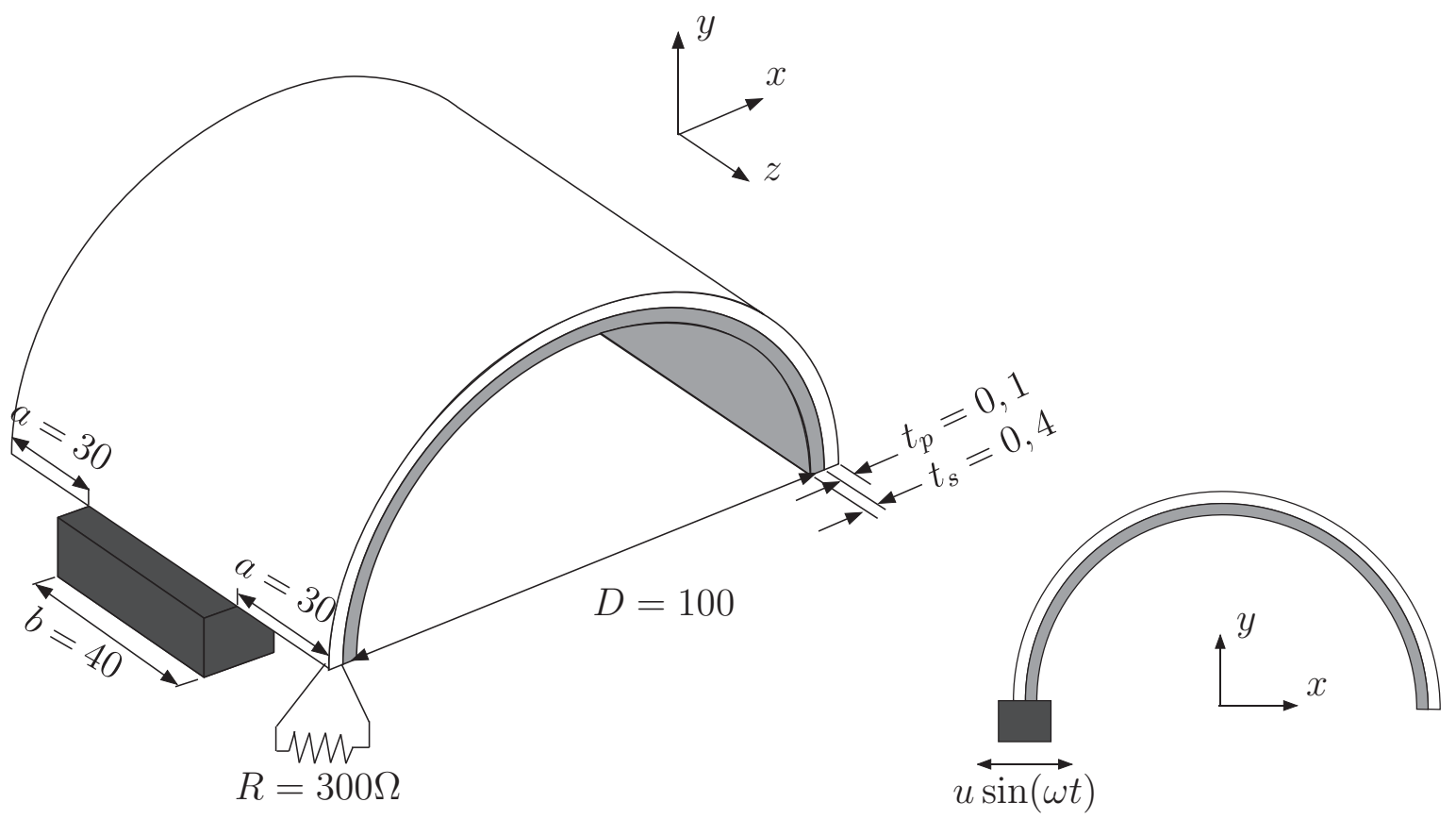

Figura 6.13: Domínio curvo de meio cilindro para o exemplo 2 do projeto de coletores de energia. Medidas em mm.

no substrato. A excitação de base tem amplitude igual a $u=1 \mathrm{~mm}$ somente na direção $x$, como mostrado na Fig. 6.13(b) e a frequência de excitação é igual a $f_{\omega}=200 \mathrm{~Hz}$ pois está próxima a uma frequência de ressonância, frequência na qual são gerados os maiores valores de potência elétrica. A frequência angular $\omega$ é igual a $\omega=2 \pi f_{\omega}$. A resistência elétrica utilizada tem valor igual a $R=300 \Omega$.

Uma análise de convergência da malha de elementos finitos revelou que a convergência dos valores de potência elétrica e de tensão mecânica é obtida quando a estrutura é dividida em 20 elementos na direção z e 30 elementos na direção circunferencial. Não foi utilizada uma malha mais refinada pois o elemento de casca multi-camadas utilizado, junto com o método OMD, são muito custosos computacionalmente.

A Fig. 6.14 apresenta a forma deformada da estrutura sem aplicar nenhuma otimização, ou seja, a camada de material piezelétrico está totalmente cheia e com polarização positiva Contudo, como a potência é uma função módulo, o sentido de polarização não faz diferença. Esse caso será chamado de caso 0, e obteve-se uma potência elétrica gerada igual a $1,13 \mathrm{~W}$.

Além disso, analisando a distribuição de tensões da Fig. 6.15, nota-se que existem regiões nas duas camadas que apresentam tensões acima do limite do material. Na camada de alumínio, o valor máximo de von Mises é igual a 513,42 MPa e na camada de material piezelétrico é igual a 347,38 MPa, ou seja, estão muito acima dos limites de escoamento do 


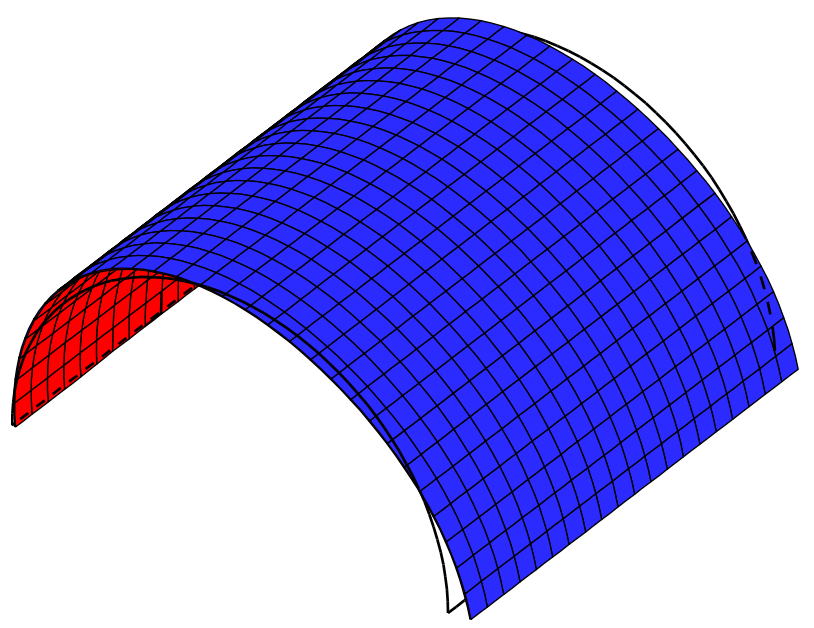

Figura 6.14: Forma deformada da estrutura para o caso 0 de coletores de energia de casca, ou seja, sem otimização, com a camada piezelétrica totalmente cheia e polarização positiva.

alumínio e de despolarização do material piezelétrico, respectivamente. Os altos valores de tensão são decorrentes do alto valor da amplitude de oscilação da estrutura, que nesse caso chega a ser igual a $8,57 \mathrm{~mm}$. Essas altas amplitudes são geradas pela vibração da grande quantidade de massa que a estrutura possui, já que o material piezelétrico tem alto valor de densidade. Assim, a restrição de falha mecânica torna-se necessária no projeto de coletores de energia com o objetivo de reduzir as amplitudes de vibração aliviando assim, as tensões na estrutura.

As Figs. 6.16(a) e 6.16(b) referem-se aos resultados de otimização de topologia e de polarização para o caso 1 e a Fig. 6.17 apresenta sua respectiva deformada pós-processada. O valor da potência elétrica obtida neste caso é igual a $0,26 \mathrm{~W}(0,22 \mathrm{~W}$ para o resultado pós-processado), 4,34 vezes menor que no caso 0. Essa redução no valor da potência elétrica é devido, principalmente, à restrição de falha mecânica que agora é obedecida. Ou seja, os valores máximos de tensão de von Mises para as camadas de alumínio e de material piezelétrico são iguais a 205,21 e 49,98 MPa, respectivamente, como podem ser observados na Fig. 6.18. A amplitude de deslocamento máxima na estrutura é igual a $1,7 \mathrm{~mm}, 2,6$ vezes menor que no caso 0. Assim, embora esse resultado produza um valor menor de potência elétrica, pode-se garantir que nenhuma das camadas ultrapassa seu respectivo limite de tensão.

O volume final da camada piezelétrica é igual a 56,55\% do volume total da camada. Nota-se na Fig. 6.16(a) que existem algumas regiões de material intermediário (cinza), ou seja, a metodologia utilizada não conseguiu eliminar essas regiões indesejáveis, mas 

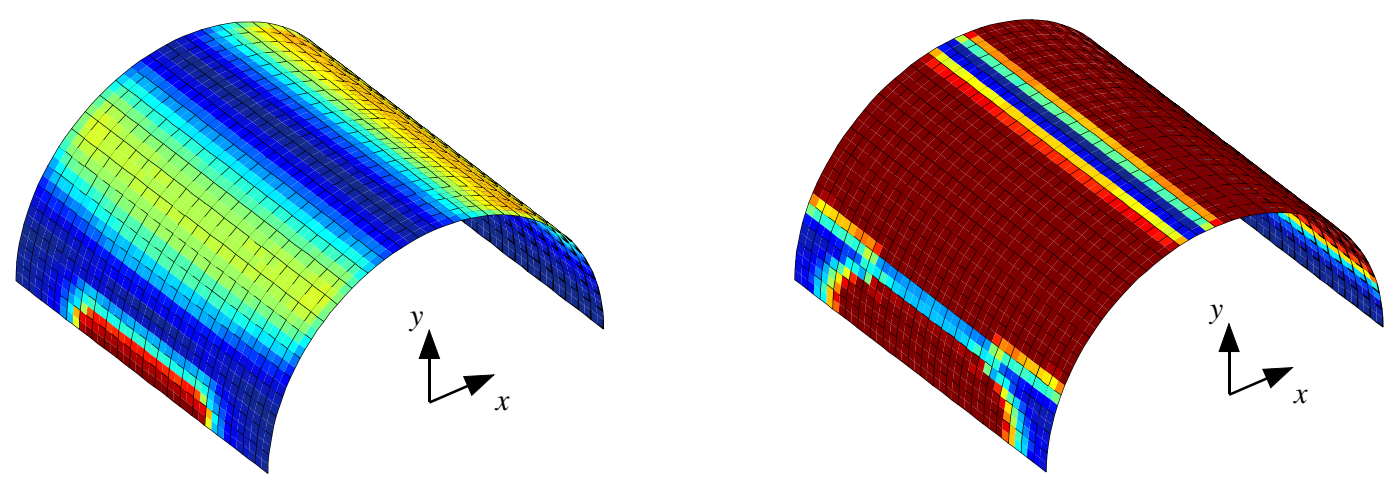

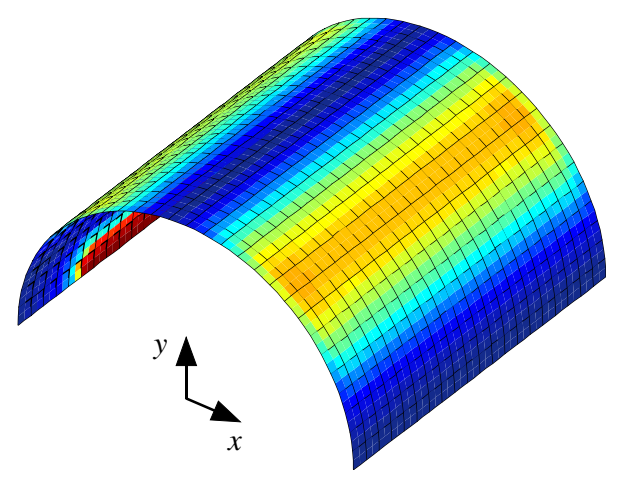

(a) Camada de alumínio

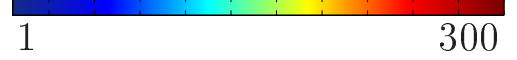

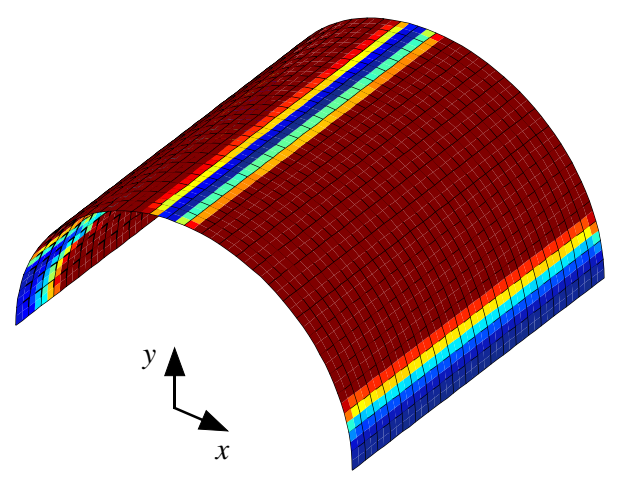

(b) Camada piezelétrica

1
50

Figura 6.15: Duas vistas da distribuição das tensões de von Mises das superfícies críticas das camadas de alumínio e de material piezelétrico para o caso 0 de coletores de energia de casca. Valores de tensão em MPa, onde os valores máximos da escala representam os limites de tensão.

conseguiu minimizar a sua ocorrência. Na Fig. 6.16(b) observa-se uma grande região de polarização intermediária (cinza), mas que coincide com as regiões onde não há material piezelétrico, podendo assim, ser desconsiderada. Na Fig. 6.18 são apresentadas as distribuições de tensão mecânica nas superfícies inferior e superior das duas camadas.

Agora para o caso 2, as Figs. 6.19(a), 6.19(b) e 6.20 referem-se aos resultados de otimização de topologia, de polarização e de orientação e a Fig. 6.21 apresenta sua respectiva deformada do resultado pós-processado.

O valor da potência elétrica obtida neste caso é igual a $0,28 \mathrm{~W}(0,21 \mathrm{~W}$ para o resultado pós-processado), 4,03 vezes menor que no caso 0 , mas $7 \%$ maior que no caso 1. Os valores de tensão mecânica são ainda menores que no caso 1, sendo 54,32 e 43,10 MPa para as camadas de alumínio e de material piezelétrico, respectivamente, e 0,16 para a camada de fibra de carbono. Ou seja, a otimização da orientação das fibras contribuiu para a redução do valor da tensão máxima na estrutura, permitindo maior quantidade de 

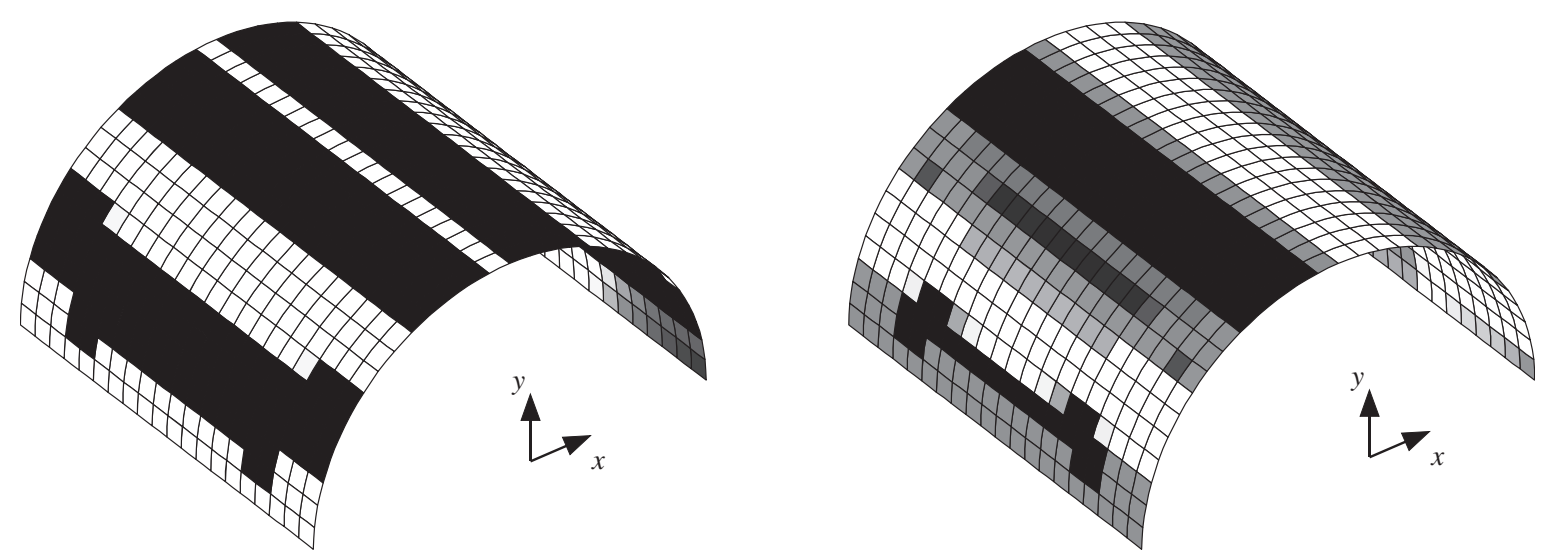

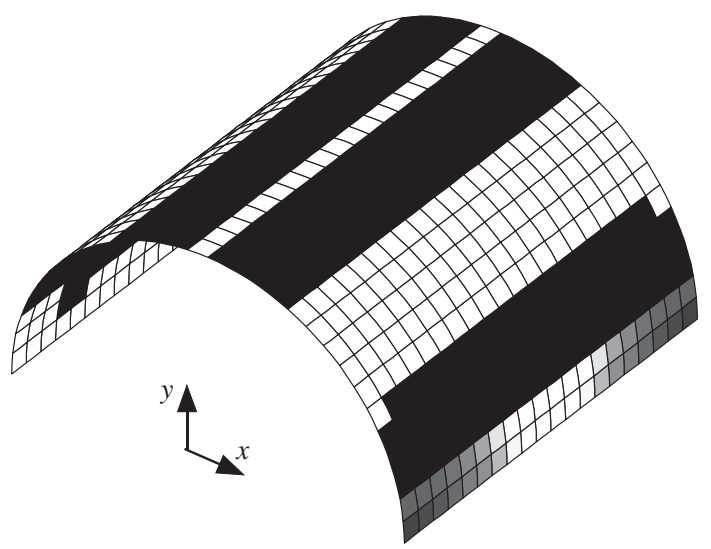

(a) Resultado de otimização topológica

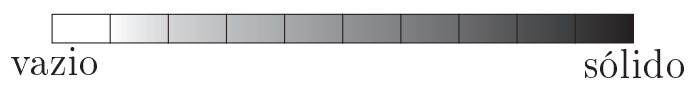

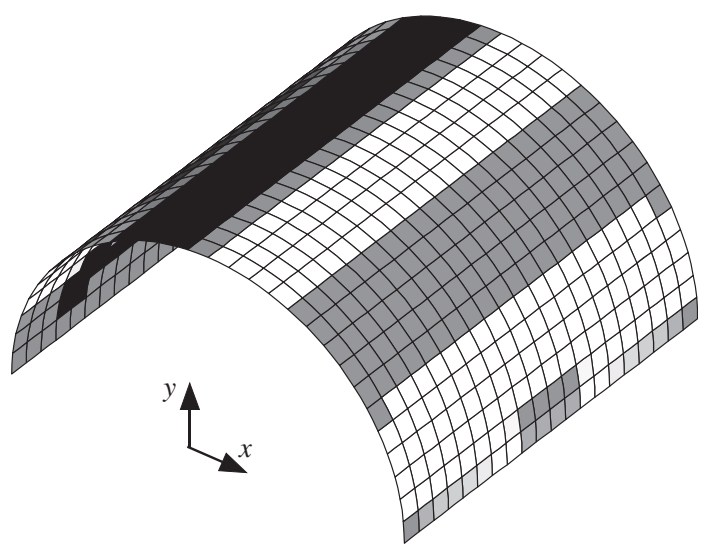

(b) Resultado de otimização de polarização

Figura 6.16: Resultado da otimização de topologia (a) e de polarização (b) das camadas piezelétricas para o caso 1 de coletores de energia de casca, representados em duas vistas.

material no resultado final, aumentando assim a potência gerada, que é maior do que no caso 1. A amplitude máxima de deslocamento na estrutura é igual a 1,7 mm, a metade do valor obtido no caso 1 . Ou seja, a estrutura ficou mais rígida que no caso 1 , mas gera mais potência.

Na Fig. 6.22 são apresentadas as distribuições de tensão mecânica nas superfícies inferior e superior das três camadas. As imagens de cima e de baixo representam as superfícies superior e inferior, respectivamente, onde os valores máximos da escala representam os limites de tensão para cada critério. Como o máximo valor de Tsai-Wu na camada e fibra de carbono é igual a 0,16 , optou-se por não apresentar a distribuição dos valores de Tsai-Wu. 


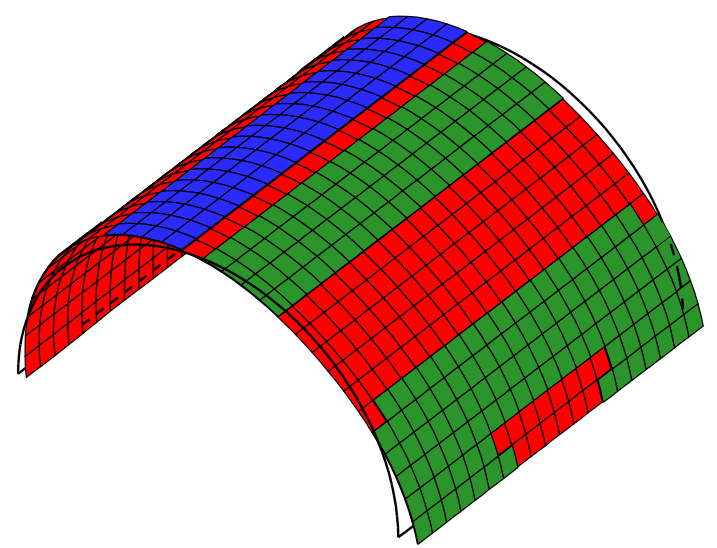

Figura 6.17: Forma deformada do resultado pós-processado para o caso 1 de coletores de energia de casca. Em vermelho está representada a camada de alumínio, e em azul e verde está representada a camada piezelétrica com polarização positiva e negativa, respectivamente.
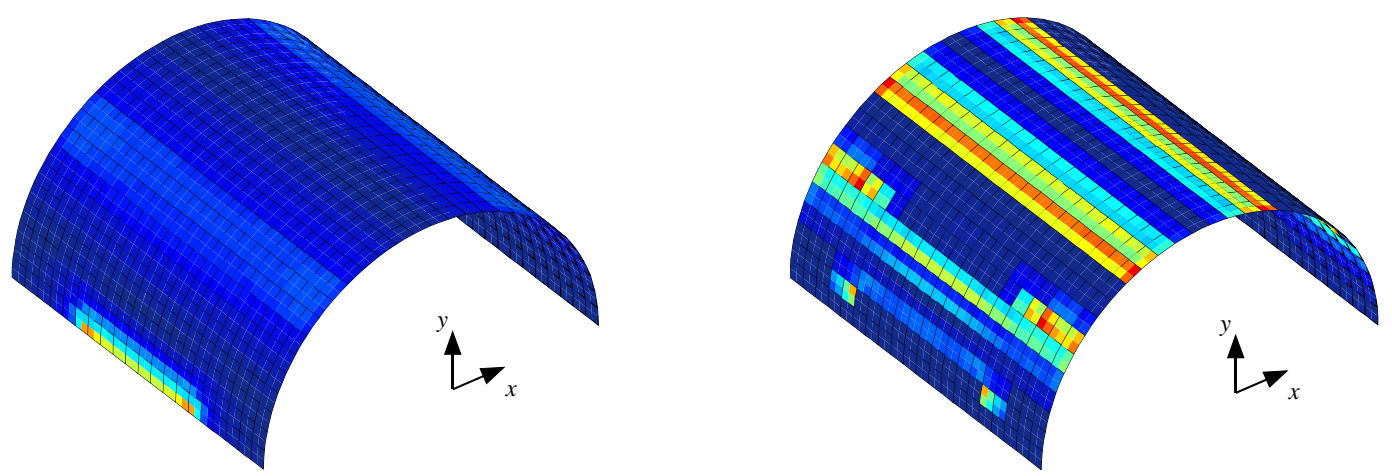

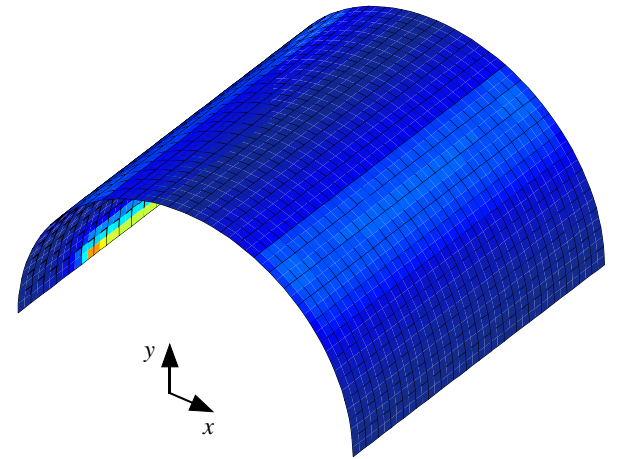

(a) Camada de alumínio

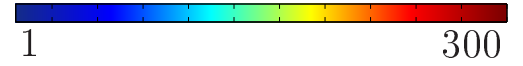

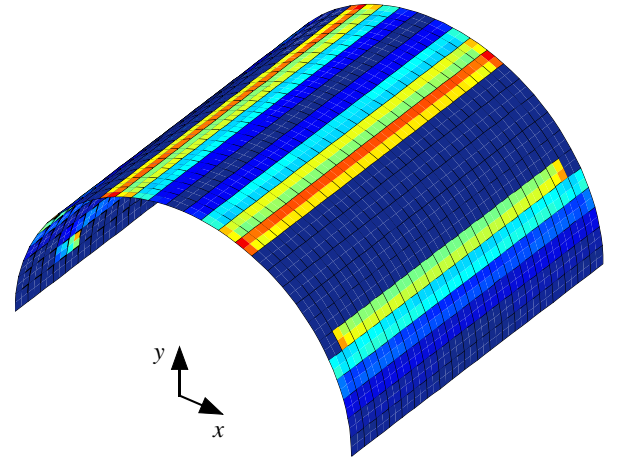

(b) Camada piezelétrica

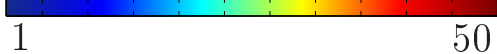

Figura 6.18: Duas vistas da distribuição das tensões de von Mises das superfícies críticas das camadas de material piezelétrico para o caso 1 de coletores de energia de casca. Valores de tensão em MPa, onde os valores máximos da escala representam os limites de tensão. 

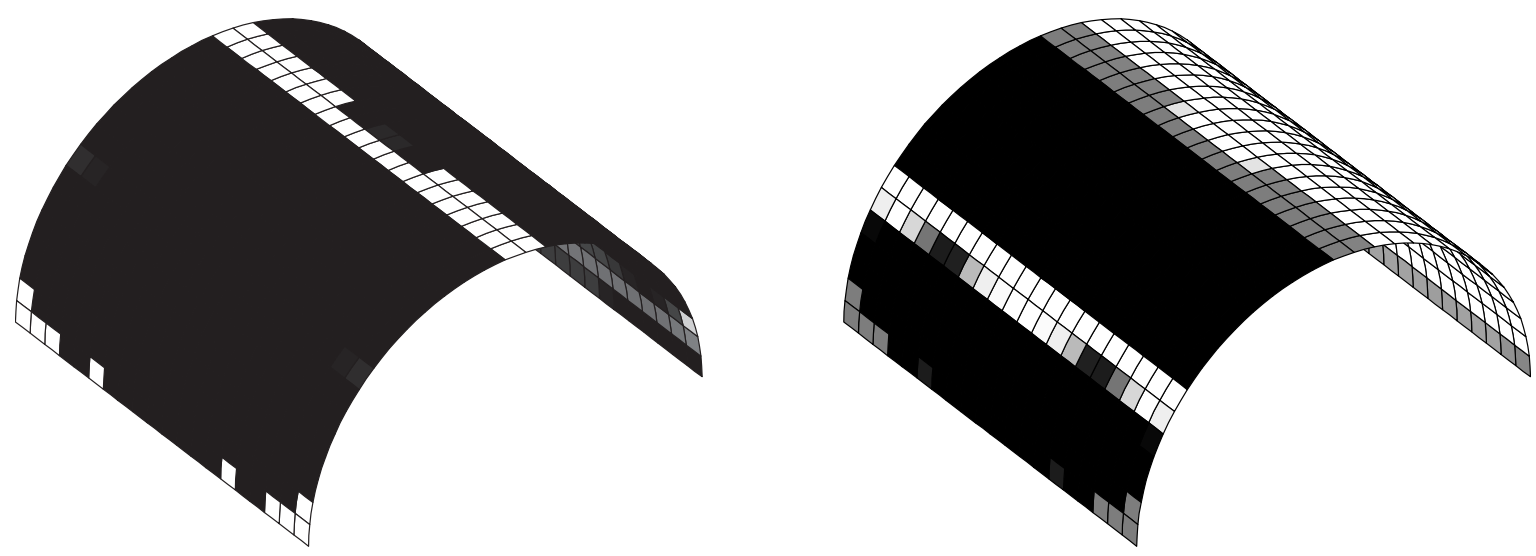

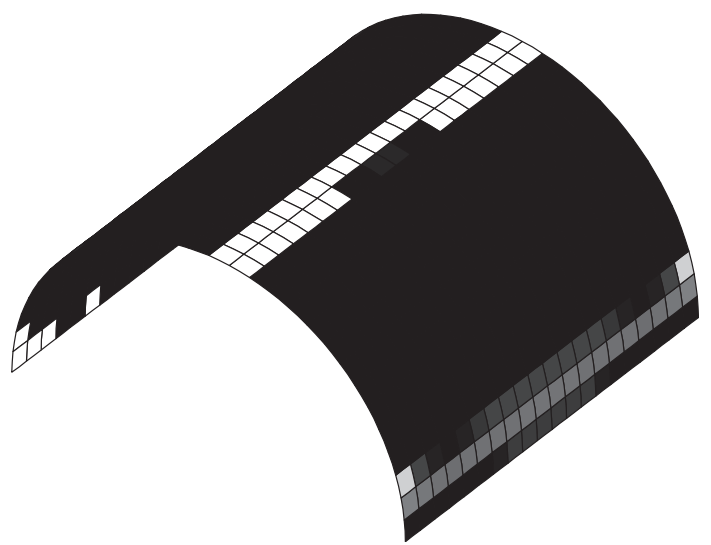

(a) Resultado de otimização topológica

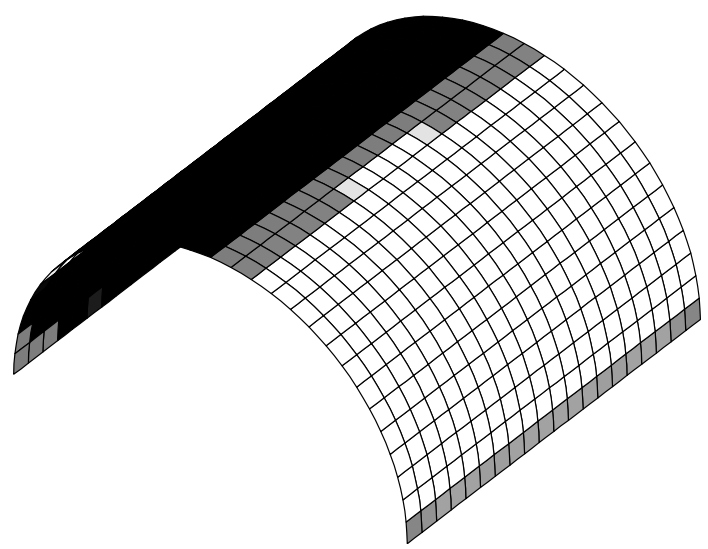

(b) Resultado de otimização de polarização

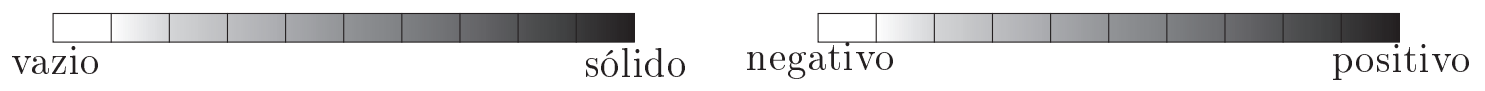

Figura 6.19: Resultado da otimização de topologia (a) e de polarização (b) das camadas piezelétricas para o caso 2 de coletores de energia de casca, representados em duas vistas.

A Tab. 6.2 apresenta os valores de potência elétrica gerada ( $\wp)$, de tensão máxima de von Mises na camada piezelétrica $\left(\sigma_{v m_{\text {max }}}^{\text {piezo }}\right)$ e na camada de alumínio $\left(\sigma_{v m_{\text {max }}}^{A l}\right)$, de tensão máxima de Tsai-Wu na camada de fibra de carbono $\left(\sigma_{t w_{\max }}^{F C}\right)$ e de volume final da camada piezelétrica otimizada $\left(V_{o t}\right)$ para todos os casos apresentados.

Tabela 6.2: Dados dos resultados apresentados para o exemplo de coletores de energia de casca.

\begin{tabular}{cccccc}
\hline & $\wp$ & $\sigma_{v m_{\max }}^{\text {piezo }}$ & $\begin{array}{c}\sigma_{v m_{\max }}^{A l} \\
\mathrm{MPa}\end{array}$ & $\sigma_{t w_{\max }}^{F C}$ & $\begin{array}{c}V_{\text {ot }} \\
\text { MPa }\end{array}$ \\
\hline caso 0 & 1,13 & 347,38 & 513,42 & - & - \\
caso 1 & 0,26 & 49,98 & 205,21 & - & 56,55 \\
caso 2 & 0,28 & 43,10 & 54,32 & 0,16 & 90,84 \\
\hline
\end{tabular}



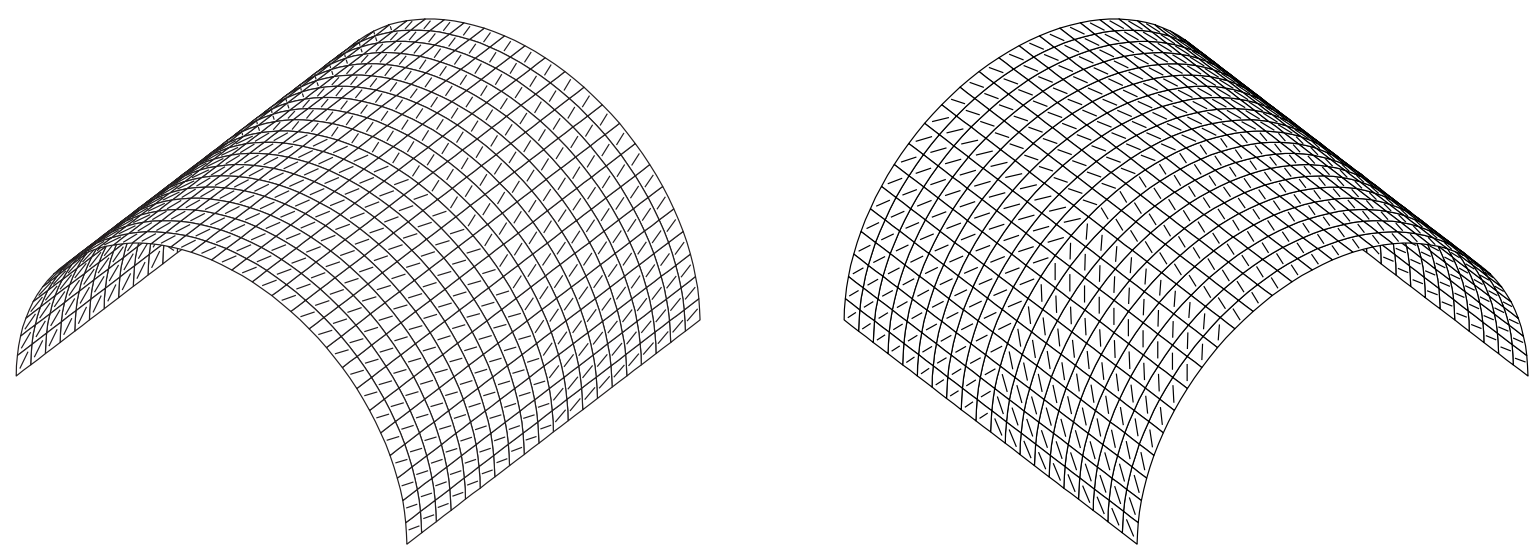

Figura 6.20: Resultado da otimização de orientação de fibras para o caso 2 de coletores de energia de casca.

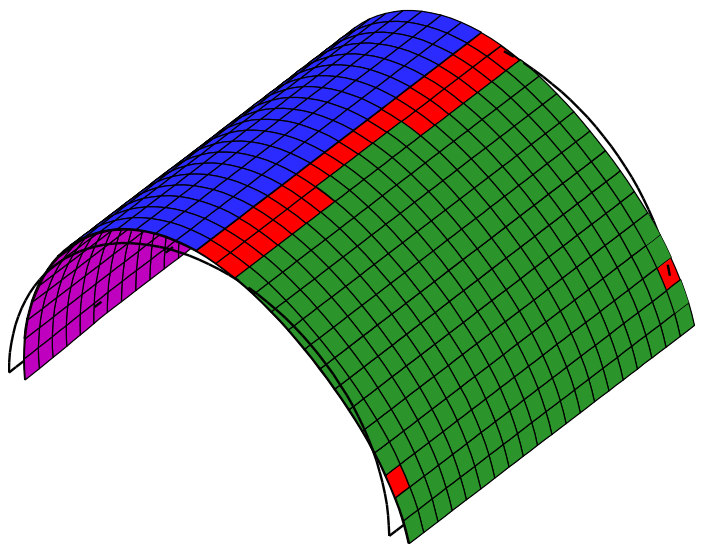

Figura 6.21: Forma deformada do resultado pós-processado para o caso 2 de coletores de energia de casca. Em vermelho está representada a camada de alumínio, e em azul e verde está representada a camada piezelétrica com polarização positiva e negativa, respectivamente.

O gráfico da Fig. 6.23 apresenta a resposta em frequência do coletor de energia. Analisando a linha azul que representa o caso 0, existe uma frequência de ressonância, cujo valor é igual a $190 \mathrm{~Hz}$, que está bem próxima à frequência de excitação de $200 \mathrm{~Hz}$. Embora o valor da potência elétrica gerada neste caso ser baixo, as deformações são altas, gerando altos valores de tensões mecânicas que ultrapassam todos os limites de tensão estabelecidos no problema, como apresentado anteriormente.

Nos casos 1 e 2, pode-se notar que a estrutura foi ajustada para que as frequências de ressonância não ficassem próximas à frequência de excitação. Esse fenômeno pode ser justificado, novamente, pela utilização da restrição de falha mecânica, que impede que as amplitudes de deslocamento tenham valores muito altos. 

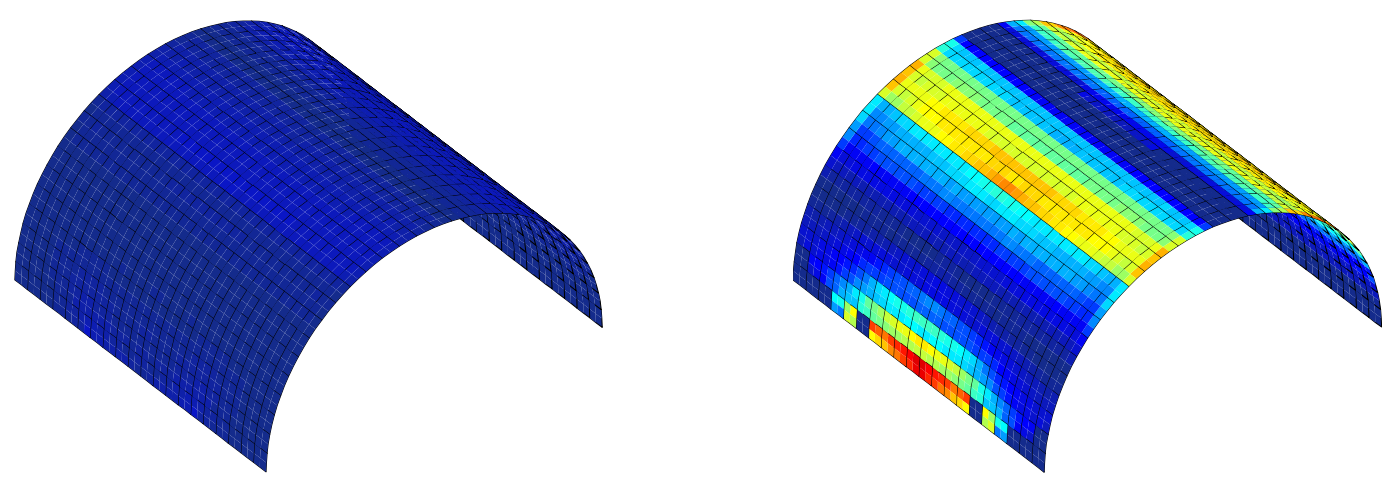

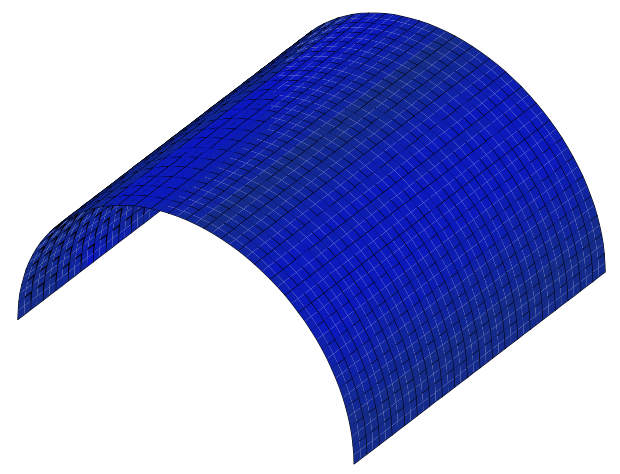

(a) Camada de alumínio

1

300

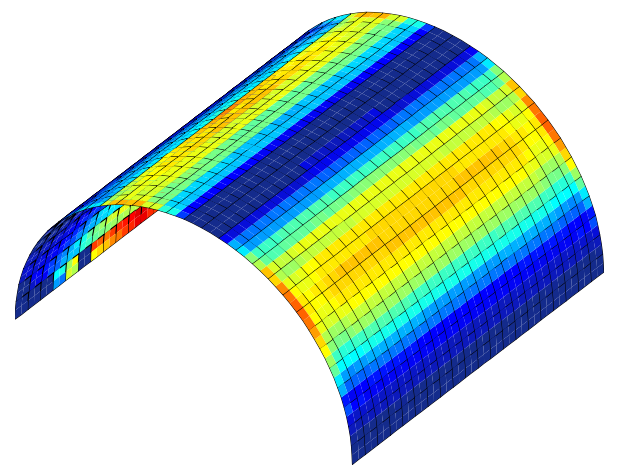

(b) Camada piezelétrica

1

Figura 6.22: Duas vistas da distribuição das tensões de von Mises da superfície crítica das camadas de material piezelétrico para o caso 2 de coletores de energia de casca. Valores de tensão em MPa, onde os valores máximos da escala representam os limites de tensão.

No caso 1, representado pela linha verde, nota-se que a frequência de ressonância caiu para, aproximadamente, $175 \mathrm{~Hz}$, e no caso 2, representado pela linha vermelha, a frequência reduziu ainda mais, e ficou igual a $140 \mathrm{~Hz}$. Na frequência de excitação de $200 \mathrm{~Hz}$, pode-se observar que os valores de potência elétrica gerados pelos casos 1 e 2 são praticamente iguais. Entretanto, pode-se verificar que no caso 2, existe uma faixa de frequência em torno da frequência de $200 \mathrm{~Hz}$ que apresenta uma boa estabilidade nos valores de potência elétrica gerada. Essa faixa normalmente é chamada de faixa de operação. Já no caso 1, essa faixa de operação fica entre 260 e $280 \mathrm{~Hz}$. Essa faixa de estabilidade é importante para que a estrutura não seja tão sensível com relação às mudanças no valor da frequência de operação, ou seja, se por algum motivo qualquer, a frequência de excitação aumenta ou diminui, o valor da potência gerada não tende a se alterar. 


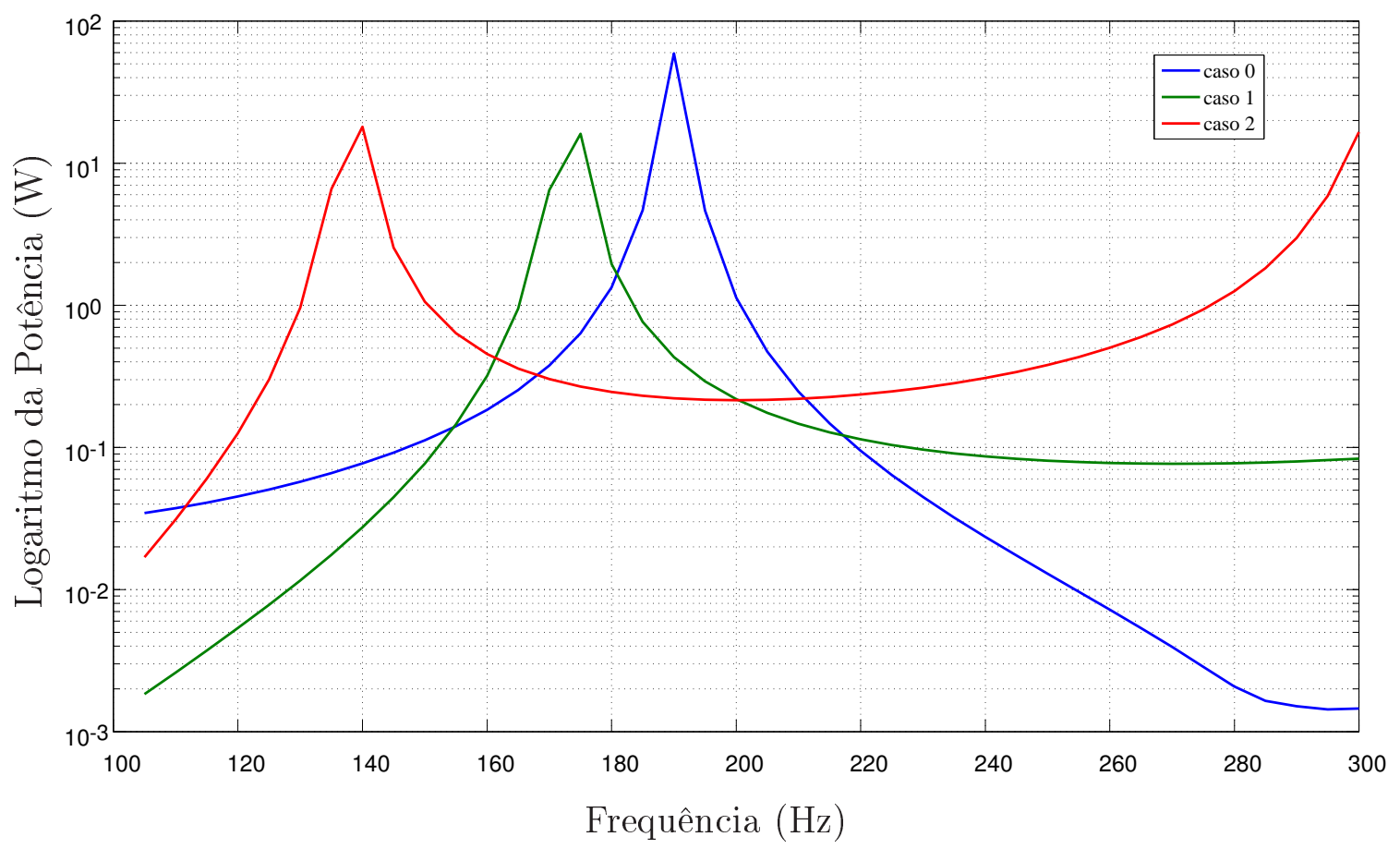

Figura 6.23: Gráfico logarítmico da resposta em frequência dos três casos apresentados anteriormente.

\subsection{Discussão}

Assim, considerando os exemplos apresentados do projeto de coletores de energia, nota-se que é necessário utilizar restrições de tensão mecânica para garantir que os valores de tensão não ultrapassem certos limites. No caso do material piezelétrico, sua tensão limite de despolarização é muito baixa, fazendo com que essa camada sempre seja a camada crítica.

A otimização de distribuição de material foi essencial para essa redução nos valores de tensão, já que reduzindo a quantidade de material piezelétrico, reduz-se as amplitudes de vibração da estrutura, aliviando as tensões. Já a otimização de polarização se mostrou importante para aproveitar as deformações de tração e contração existentes no modo em que a estrutura vibra. Finalmente, a otimização de orientação de fibras contribuiu para que a estrutura ficasse mais rígida em algumas regiões, permitindo maior volume de material e, consequentemente, maior geração de potencial elétrico e portanto, de energia elétrica, com tensões mecânicas reduzidas. 


\section{CONCLUSÕES}

Utilizando MOT, foi desenvolvida uma metodologia genérica para o projeto de transdutores piezocompósitos de casca multi-camada considerando a otimização simultânea de distribuição e polarização de material piezelétrico e da orientação das fibras do material ortotrópico, sujeita à uma restrição global de tensão mecânica. A metodologia proposta foi utilizada para aplicações de atuadores, sensores e coletores de energia.

No caso de atuadores, foi visto que a restrição de falha mecânica não é necessária, já que os deslocamentos são pequenos e podem ser controlados pelo potencial elétrico aplicado. A utilização do modelo PEMAP-P junto com a técnica de projeção com a função "degrau" se mostrou bastante eficaz na obtenção de resultados praticamente discretos, ou seja, com distribuição 0-1. Também foi visto que, ao otimizar simultaneamente a distribuição de material junto com o sentido de polarização, foram criadas topologias com sentido de polarização opostos na mesma camada. Isso significa que, ao aplicar o mesmo potencial, algumas regiões devem se contrair, e outras estender, melhorando assim o controle dos deslocamentos gerados. Além disso, a otimização da orientação das fibras se mostrou uma opção para deixar o substrato mais flexível sem ter que retirar material dele, e assim, maximizando os deslocamentos desejados.

No caso de sensores, foi mostrado que a restrição de falha mecânica é essencial para se obter uma topologia ótima que respeite os limites de tensão de cada tipo de material. Foi observado também que a otimização da polarização auxilia na maximização do potencial elétrico já que podem existir regiões com deformação de tração e de compressão na estrutura deformada. Já a otimização das fibras faz com que as regiões com maiores deformações fiquem mais rígidas, resultando em valores de tensão mecânicas abaixo dos limites, e para as regiões de baixa deformação, a camada de fibra de carbono tende a ficar mais flexível, possibilitando maior geração de potencial elétrico.

Já no caso dos coletores de energia, a adequada distribuição de material e de orientação de fibras foram essenciais para a redução dos valores de tensão mecânica na estrutura, uma vez que no problema dinâmico tem-se o fenômeno da ressonância, ou seja, são 
frequências na qual a estrutura vibra com amplitude máxima. Assim, a OT neste caso distribui o material e orienta as fibras adequadamente para que a vibração da estrutura seja controlada a níveis aceitáveis de tensão, e força que exista uma faixa de operação estável próxima à frequência de excitação. Já a polarização, novamente, se adequa à deformações da estrutura de forma a aproveitar tanto as deformações de tração quanto as de compressão.

Os resultados estáticos de atuadores e sensores apresentaram topologias praticamente discretas, ou seja, 0-1. Assim, pôde se comprovar a eficácia da combinação do modelo de material PEMAP-P (KÖGL; SILVA, 2005) (com escolha adequada dos coeficientes de penalização) com a técnica de projeção com a função "degrau", proposta por Guest, Asadpoure e Ha (2011). Nesse estudo entretanto, os resultados dinâmicos de coletores de energia apresentaram algumas regiões de valores intermediários de pseudodensidade. Notou-se que essas regiões de pseudodensidade intermediárias são decorrentes da instabilidade do problema dinâmico, já que este pode envolver modos naturais de vibração. Entretanto neste trabalho mostrou-se que o uso adequado dos coeficientes de penalização pode minimizar o aparecimento das pseudodensidades intermediárias.

Assim, este trabalho traz uma abordagem inovadora no projeto de transdutores piezocompósitos de casca multicamada.

\subsection{Sugestão de Trabalhos Futuros}

Como principal sugestão de trabalhos futuros, pode ser implementada uma formulação para a caracterização experimental dos transdutores aqui mencionados, otimizados utilizando a metodologia proposta nesta tese. Entretanto, como foi dito no capítulo de atuadores, algumas restrições de manufatura devem ser consideradas, como por exemplo, o agrupamento de regiões com a mesma orientação de fibras, já que métodos atualmente disponíveis não conseguem altas discretizações de elementos.

Quanto à geometria, pode-se inicialmente projetar transdutores de placas, utilizando cerâmicas piezelétricas planas, que são facilmente encontradas. Em seguida, para fabricar os transdutores com superfícies curvas, sugere-se utilizar os materiais piezelétricos fibrosos (WILKIE et al., 2006; PARK et al., 2010a; KIM; SOHN; CHOI, 2011), ou "Macro Fiber Composite" (MFC) como são conhecidos na literatura. Esses materiais podem ser colados em estruturas curvas pois são flexíveis, como mostra a Fig. 7.1 de um MFC.

Entretanto, esses materiais não foram utilizados nesta tese pois não há na literatura 


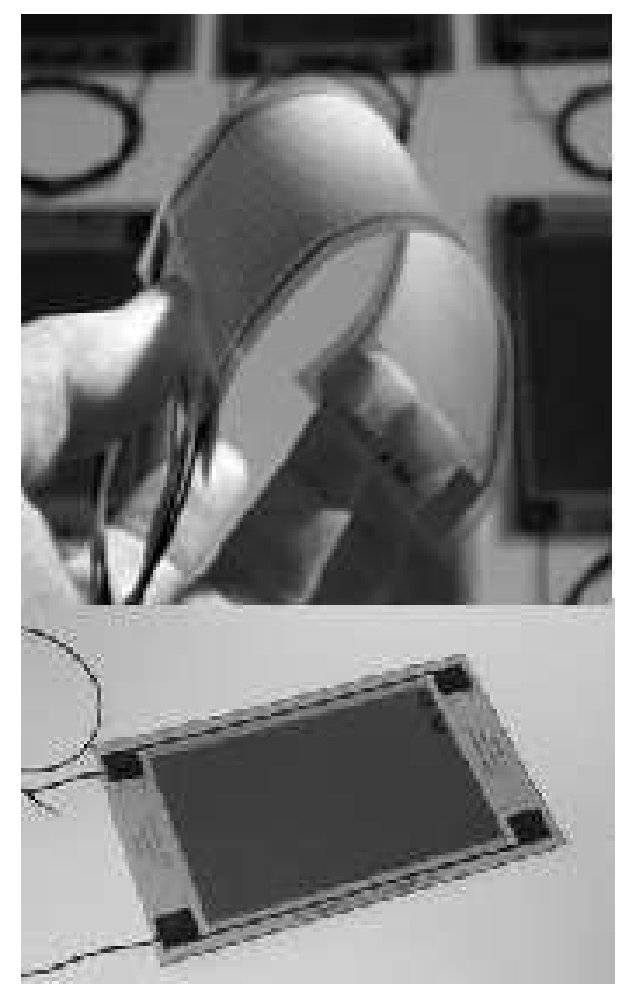

Figura 7.1: Materiais piezelétricos fibrosos, ou MFC.

um trabalho sobre a caracterização das propriedades desse tipo de material. O que é comumente utilizado, são propriedades homogeneizadas misturando-se as propriedades de um material piezelétrico com as de uma resina. Nesse sentido, sugere-se que se faça uma validação de resultados numéricos e experimentais de uma estrutura simples para avaliar a diferença que o modelo numérico pode apresentar, e então utilizá-lo na otimização e na fabricação de protótipos otimizados. 


\section{Referências}

AGRAWAL, B. N.; TREANOR, K. E. Shape control of a beam using piezoelectric actuators. Smart Materials \& Structures, Iop Publishing Ltd, v. 8, n. 6, p. 729-739, 1999.

AHMAD, S.; IRONS, B.; ZIENKIEWICZ, O. Analysis of thick and thin shell structures by curved elements. International Journal for Numerical Methods in Engineering, v. 2, p. 419-451, 1970.

ALLAIRE, G.; JOUVE, F.; MAILLOT, H. Topology optimization for minimum stress design with the homogenization method. Structural and Multidisciplinary Optimization, Springer, v. 28, n. 2, p. 87-98, 2004.

ALLAIRE, G.; JOUVE, F.; TOADER, A. M. Structural optimization using sensitivity analysis and a level-set method. Journal of Computational Physics, v. 194, n. 1, p. 363-393, fev. 2004.

ALLIK, H.; HUGHES, T. J. R. Finite element method for piezoelectric vibration. International Journal for Numerical Methods in Engineering, v. 2, p. 151-157, 1970.

AMBROSIO, L.; BUTTAZZO, G. An optimal-design problem with perimeter penalization. Calculus of Variations and Partial Differential Equations, v. 1, n. 1, p. 55-69, jan. 1993.

ANDRADE, M. A. B.; BUIOCHI, F.; ADAMOWSKI, J. C. Finite element analysis and optimization of a single-axis acoustic levitator. IEEE Transactions on Ultrasonics, Ferroelectrics, and Frequency Control, v. 57, n. 2, p. 469-479, 2010.

ANTON, S. R.; SODANO, H. A. A review of power harvesting using piezoelectric materials (2003-2006). Smart Materials $\&$ Structures, v. 16, p. R1-R21, 2007.

BALAMURUGAN, V.; NARAYANAN, S. Shell finite element for smart piezoelectric composite plate/shell structures and ills application to the study of active vibration control. Finite Element in Analysis and Design, v. 35, n. 9, p. 713-738, 2001.

BALAMURUGAN, V.; NARAYANAN, S. A piezolaminated composite degenerated shell finite element for active control of structures with distributed piezosensors and actuators. Smart Materials and Structures, v. 17, n. 3, 2008.

BATHE, K. J. Finite Element Procedures. 1. ed. New Jersey: Prentice Hall, 1996.

BEEBY, S. P.; TUDOR, M. J.; WHITE, N. M. Energy harvesting vibration sources for microsystems applications. Measurement Science and Technology, v. 13, p. 175-195, 2006.

BENDSØE, M. P. Optimal shape design as a material distribution problem. Structural Optimization, v. 1, p. 192-202, 1989. 
BENDSØE, M. P.; KIKUCHI, N. Generating optimal topologies in structural design using a homogenization method. Computer Methods In Applied Mechanics and Engineering, v. 71, n. 2, p. 197-224, nov. 1988.

BEnDSØE, M. P.; SIGMUND, O. Topology Optimization - Theory, Methods and Application. 2. ed. Berlin: Springer, 2003.

BOURDIN, B. Filters in topology optimization. International Journal for Numerial Methods in Engineering, v. 50, n. 9, p. 2143-2158, mar. 2001.

BREI, D.; MOSKALIK, A. J. Deflection performance of a bi-directional distributed polymeric piezoelectric micromotor. Journal of Microelectromechanical Systems, v. 6, n. 1, p. 62-69, 1997.

BRUGGI, M. On an alternative approach to stress constraints relaxation in topology optimization. Structural and multidisciplinary optimization, Springer, v. 36, n. 2, p. 125-141, 2008.

BRUGGI, M.; VENINI, P. A mixed FEM approach to stress-constrained topology optimization. International Journal For Numerical Methods In Engineering, v. 73, n. 12, p. 1693-1714, mar. 2008.

BRUNS, T. E.; TORTORELLI, D. A. Topology optimization of non-linear elastic structures and compliant mechanisms. Computer Methods In Applied Mechanics and Engineering, v. 190, n. 26-27, p. 3443-3459, 2001.

CALDERON-MORENO, J. Stress induced domain switching of PZT in compression tests. Materials Science and Engineering: A, Elsevier, v. 315, n. 1, p. 227-230, 2001.

CARBONARI, R. C. Projeto de multi-atuadores piezelétricos homogêneos e gradados utilizando o método de otimização topológica. Tese (Doutorado) - Escola Politécnica da USP, 2008.

CARBOnARI, R. C.; SILVA, E. C. N.; NISHIWAKI, S. Design of piezoelectric multi-actuated microtools using topology optimization. Smart Materials 85 Structures, v. 14, n. 6, p. 1431-1447, 2005.

CARBONARI, R. C.; SILVA, E. C. N.; NISHIWAKI, S. Optimum placement of piezoelectric material in piezoactuator design. Smart Materials 6 Structures, v. 16, n. 1, p. 207-220, 2007.

CHEN, S.; GONELLA, S.; CHEN, W.; LIU, W. A level set approach for optimal design of smart energy harvesters. Computer Methods in Applied Mechanics and Engineering, Elsevier, v. 199, n. 37, p. 2532-2543, 2010.

CHENG, G.; JIANG, Z. Study on topology optimization with stress constraints. Engineering Optimization, Taylor \& Francis, v. 20, n. 2, p. 129-148, 1992.

CHENG, G. D.; GUO, X. Epsilon-relaxed approach in structural topology optimization. Structural Optimization, v. 13, n. 4, p. 258-266, jun. 1997. 
COOK-CHENnAUlT, K. A.; THAMBI, N.; SASTRY, A. M. Powering mems portable devices - a review of non-regenerative and regenerative power suply systems with emphasis on piezoelectric energy harvesting systems. Smart Materials $\&$ Structures, v. 17 , p. 1-33, 2008.

COOK, R.; MALKUS, D.; PLESHA, M.; WITT, R. Concepts and applications of finite element analysis. [S.1.]: John Wiley \& Sons, 2007.

DESILETS, C.; WOJCIK, G.; NIKODYM, L.; MESTERTON, K. Analyses and measurements of acoustically matched, air-coupled tonpilz transducers. IEEE Ultrasonics Symposium, v. 1, p. 1045-1048, 1999.

DIAZ, A.; SIGMUND, O. Checkerboard patterns in layout optimization. Structural Optimization, v. 10, n. 1, p. 40-45, ago. 1995.

DIAZ, A. R.; KIKUCHI, N. Solutions to shape and topology eigenvalue optimization problems using a homogenization method. International Journal For Numerical Methods In Engineering, John Wiley \& Sons Ltd, v. 35, n. 7, p. 1487-1502, 1992.

DUTOIT, N. E.; WARDLE, B. L.; KIM, S. G. Design considerations for mems-scale piezoelectric mechanical vibration energy harvesters. Integrated Ferroelectrics, v. 71, p. 121-160, 2005.

DUVAUT, G.; TERREL, G.; LENE, F.; VERIJENKO, V. E. Optimization of fiber reinforced composites. Composite Structures, v. 48, n. 1-3, p. 83-89, 2000.

DUYSINX, P.; BENDSØE, M. P. Topology optimization of continuum structures with local stress constraints. International Journal For Numerical Methods In Engineering, v. 43, n. 8, p. 1453-1478, 1998.

EECHENAUER, H. A.; KOBELEV, V. V.; SCHUMACHER, A. Bubble method for topology and shape optimization of structures. Structural Optimization, v. 8, n. 1, p. 42-51, ago. 1994.

ERTURK, A.; INMAN, D. J. A distributed parameter electromechanical model for cantilevered piezoelectric energy harvesters. Journal of Vibration and Acoustics, v. 130, p. $1-15,2008$.

ERVIN, J.; BREI, D. Recursive piezoelectric-strain-amplifying actuator architecture. IEEE/ASME Transactions on Mechatronics, v. 3, p. 293-301, 1998.

FRECKER, M. I. Recent advances in optimization of smart structures and actuators. Journal Of Intelligent Material Systems And Structures, Sage Publications Ltd, v. 14, n. 4-5, p. 207-216, 2003.

FRECKER, M. I.; ANANTHASURESH, G. K.; NISHIWAKI, S.; KIKUCHI, N.; KOTA, S. Topological synthesis of compliant mechanisms using multi-criteria optimization. Journal of Mechanical Design, v. 119, n. 2, p. 238-245, 1997.

GAIN, A. L.; PAUlinO, G. H. Phase-field based topology optimization with polygonal elements: a finite volume approach for the evolution equation. Structural and Multidisciplinary Optimization, v. 46, n. 3, p. 327-342, set. 2012. 
GROENWOLD, A.; HAFTKA, R. Optimization with non-homogeneous failure criteria like tsai-wu for composite laminates. Structural And Multidisciplinary Optimization, v. 32, p. 183-190, 2006.

GUEST, J.; PREVOST, J.; BELYTSCHKO, T. Achieving minimum length scale in topology optimization using nodal design variables and projection functions. International Journal for Numerical Methods in Engineering, v. 61, n. 2, p. 238-254, 2004. ISSN 0029-5981.

GUEST, J. K.; ASADPOURE, A.; HA, S. H. Eliminating beta-continuation from heaviside projection and density filter algorithms. Structural and Multidisciplinary Optimization, v. 44, n. 4, p. 443-453, out. 2011.

HAAR, G. Therapeutic applications of ultrasound. Progress in Biophysics and Molecular Biology, v. 93, p. 111-129, 2007.

HAFTKA, R. T.; GURDAL, Z.; KAMAT, M. P. Elements of Structural Optimization. 2. ed. [S.l.]: Kluwer Academic Publishers, 1990.

HANSEL, W.; BECKER, W. Layerwise adaptive topology optimization of laminate structures. Engineering computations, v. 16, n. 7, p. 841-851, 1999.

HOWARD, M.; PAJOT, J.; MAUTE, K.; DUNN, M. L. A computational design methodology for assembly and actuation of thin-film structures via patterning of eigenstrains. Journal of Microelectromechanical Systems, v. 18, n. 5, p. 1137-1148, 2009.

IKEDA, T. Fundamentals of Piezoelectricity. [S.1.]: Oxford University Press, 1990.

JOG, C. S.; HABER, R. B. Stability of finite element models for distributed-parameter optimization and topology design. Computer Methods in Applied Mechanics and Engineering, v. 130, p. 203-226, 1996.

JOHANSEN, L.; LUND, E. Optimization of laminated composite structures using delamination criteria and hierarchical models. Structural and Multidisciplinary Optimization, Springer, v. 38, n. 4, p. 357-375, 2009.

KANG, Z.; TONG, L. Y. Integrated optimization of material layout and control voltage for piezoelectric laminated plates. Journal of Intelligent Material Systems and Structures, v. 19, n. 8 , p. 889-904, 2008.

KANG, Z.; TONG, L. Y. Topology optimization-based distribution design of actuation voltage in static shape control of plates. Computers $\&$ Structures, v. 86, n. 19-20, p. 1885-1893, 2008.

KANG, Z.; WANG, X. Topology optimization of bending actuators with multilayer piezoelectric material. Smart Materials and Structures, IOP Publishing, v. 19, n. 7, p. $075018,2010$.

KIM, H.; SOHN, J.; CHOI, S. Vibration control of a cylindrical shell structure using macro fiber composite actuators. Mechanics based design of structures and machines, Taylor \& Francis, v. 39, n. 4, p. 491-506, 2011. 
KIM, J.; KIM, D.; MA, P.; KIM, Y. Multi-physics interpolation for the topology optimization of piezoelectric systems. Computers Methods in Applied Mechanics and Engineering, v. 199, p. 3153-3168, 2010.

KIRSCH, U. On singular topologies in optimum structural design. Structural and Multidisciplinary Optimization, Springer, v. 2, n. 3, p. 133-142, 1990.

KIYONO, C.; NAKASONE, P.; YOO, J.; MELLO, L.; SILVA, E. Optimization of hdd-sliders with piezoelectric actuation. Smart Materials and Structures.

KIYONO, C. Y.; NAKASONE, P. H.; SILVA, E. C. N. Design of piezoelectric laminated shell structures with material gradation and fiber orientation using topology optimization. In: $13^{\text {th }}$ AIAA-MAO Conference. American Institute of Aeronautics and Astronautics. Forth Worth, Texas, USA, 2010.

KIYONO, C. Y.; SILVA, E. C. N. Topology optimization method applied to the design of piezoelectric laminated composite shell structures. In: $11^{\text {th }}$ US National Congress on Computational Mechanics. UNSCCM. Minnesota, Minneapolis, USA, 2011.

KIYONO, C. Y.; SILVA, E. C. N.; REDDY, J. N. Design of laminated piezocomposite shell transducers with arbitrary fiber orientation using topology optimization approach. International Journal for Numerical Methods in Engineering, v. 90, n. 12, p. 1452-1484, 2012 .

KÖGL, M.; BUCALEM, M. L. Analysis of smart laminates using piezoelectric mitc plate and shell elements. Computers \& Structures, v. 83, n. 15-16, p. 1153-1163, 2005.

KÖGL, M.; BUCALEM, M. L. A family of piezoelectric mitc plate elements. Computers \&5 Structures, v. 83, n. 15-16, p. 1277-1297, 2005.

KÖGL, M.; SILVA, E. C. N. Topology optimization of smart structures: design of piezoelectric plate and shell actuators. Smart Materials $\&$ Structures, v. 14, n. 2, p. 387-399, 2005.

LE, C.; NORATO, J.; BRUNS, T.; HA C. TORTORELLI, D. Stress-based topology optimization for continua. Structural And Multidisciplinary Optimization, v. 41, p. 605-620, 2010.

LI, D. S.; CHENG, L. The design of synthesized structural acoustic sensors for active control of interior noise with experimental validation. Journal of Sound and Vibration, v. 329, n. 2, p. 123-139, 2010.

LI, Z.; YANG, X.; LI, Z. Application of cement-based piezoelectric sensors for monitoring traffic flows. Journal of transportation engineering, American Society of Civil Engineers, v. 132 , n. 7 , p. 565-573, 2006.

LINDGAARD, E.; LUND, E. A unified approach to nonlinear buckling optimization of composite structures. Computers \&5 Structures, v. 89, n. 3-4, p. 357-370, 2011.

LUND, E. Buckling topology optimization of laminated multi-material composite shell structures. Composite Structures, v. 91, n. 2, p. 158-167, 2009. 
MA, Z. D.; KIKUCHI, N.; CHENG, H. C. Topological design for vibrating structures. Computer Methods In Applied Mechanics And Engineering, Elsevier Science Sa Lausanne, v. 121, n. 1-4, p. 259-280, 1995.

MELLO, L.; KIYONO, C.; NAKASONE, P.; SILVA, E. On the design of quasi-static piezoelectric plate based transducers using the topology optimization method. Journal of Intelligent Material Systems and Structures, Submetido em julho de 2012.

MUKHERJEE, A.; JOSHI, S. Piezoelectric sensor and actuator spatial design for shape control of piezolaminated plates. AIAA Journal, Amer Inst Aeronaut Astronaut, v. 40, n. 6 , p. $1204-1210,2002$.

NAKASONE, P. Projeto de Transdutores Baseados em Placas Piezelétricas através do Método de Otimização Topológica. Tese (Doutorado) — Escola Politécnica da USP, 2010.

NAKASONE, P. H.; SILVA, E. C. N. Dynamic design of piezoelectric laminated sensors and actuators using topology optimization. Journal of Intelligent Material Systems and Structures, v. 21, n. 16, p. 1627-1652, nov. 2010.

NISHIWAKI, S.; FRECKER, M. I.; MIN, S.; KIKUCHI, N. Topology optimization of compliant mechanisms using the homogenization method. International Journal For Numerical Methods In Engineering, v. 42, n. 3, p. 535-559, 1998.

NISHIWAKI, S.; MIN, S.; YOO, J.; KIKUCHI, N. Optimal structural design considering flexibility. Computer Methods In Applied Mechanics and Engineering, v. 190, n. 34, p. 4457-4504, 2001.

NOVOTnY, A. A.; FEIJOO, R. A.; TAROCO, E.; PADRA, C. Topological sensitivity analysis. Computer Methods In Applied Mechanics and Engineering, v. 192, n. 7-8, p. 803-829, 2003.

ODEN, J.; KIKUCHI, N.; SONG, Y. Penalty-finite element methods for the analysis of stokesian flows. Computer Methods in Applied Mechanics and Engineering, Elsevier, v. 31, n. 3, p. 297-329, 1982.

PARK, K.; YOON, B.; NGUYEN, N.; GOO, N.; KANG, T.; YOON, K. Piezo-composite actuated control surface system for flying vehicle. Aircraft Engineering and Aerospace Technology, Emerald Group Publishing Limited, v. 82, n. 6, p. 372-375, 2010.

PARK, K. I.; XU, S.; LIU, Y.; HWANG, G. T.; KANG, S. J. L.; WANG, Z. L.; LEE, K. J. Piezoelectric batio3 thin film nanogenerator on plastic substrates. Nano Letters, v. 10, n. 12, p. 4939-4943, 2010.

Performance Composite Ltd. Mechanical Properties of Carbon fibre Composite Materials. Disponível em:

$<$ http://www.performance-composites.com/carbonfibre/mechanicalproperties_2.asp $>$.

PRIYA, S. . Advances in energy harvesting using low profile piezoelectric transducers. Journal of Electroceramics, v. 19, p. 167-184, 2007.

RAY, M. C.; REDDY, J. N. Optimal control of thin circular cylindrical laminated composite shells using active constrained layer damping treatment. Smart Materials \&6 Structures, v. 13, n. 1, p. 64-72, 2004. 
RAY, M. C.; REDDY, J. N. Performance of piezoelectric fiber-reinforced composites for active structural-acoustic control of laminated composite plates. IEEE Transactions On Ultrasonics Ferroelectrics and Frequency Control, v. 51, n. 11, p. 1477-1490, 2004.

RAY, M. C.; REDDY, J. N. Active control of laminated cylindrical shells using piezoelectric fiber reinforced composites. Composites Science and Technology, v. 65, n. 7-8, p. 1226-1236, 2005.

REDDY, J. N. An Introduction to Nonlinear Finite Element Analysis. [S.1.]: Oxford University Press, Oxford, U.K., 2004.

REDDY, J. N. An Introduction to the Finite Element Method. Third. [S.l.]: McGraw-Hill, NY, 2006.

RIVA, M.; SANZO, D. D.; AIROLDI, A.; SALA, G.; ZERBI, F. Smart structures for deformable mirrors actuated by piezocomposites. In: INTERNATIONAL SOCIETY FOR OPTICS AND PHOTONICS. SPIE Astronomical Telescopes and Instrumentation: Observational Frontiers of Astronomy for the New Decade. [S.1.], 2010. p. 77391N-77391N.

ROZVANY, G. On design-dependent constraints and singular topologies. Structural and Multidisciplinary Optimization, Springer, v. 21, n. 2, p. 164-172, 2001.

ROZVANY, G.; SOBIESZCZANSKI-SOBIESKI, J. New optimality criteria methods: forcing uniqueness of the adjoint strains by corner-rounding at constraint intersections. Structural and Multidisciplinary Optimization, Springer, v. 4, n. 3, p. 244-246, 1992.

ROZVANY, G. I. N.; ZHOU, M.; BIRKER, T. Generalized shape optimization without homogenization. Structural Optimization, v. 4, n. 3-4, p. 250-252, out. 1992.

RUPP, C. J.; DUNN, M. L.; MAUTE, K. Analysis of piezoelectric energy harvesting systems with non-linear circuits using the harmonic balance method. Journal of Intelligent Material Systems and Structures, v. 21, p. 1383-1396, 2010.

RUPP, C. J.; EVGRAFOV, A.; MAUTE, K.; DUNN, M. L. Design of piezoelectric energy harvesting systems: A topology optimization approach based on multilayer plates and shells. Journal of Intelligent Material Systems and Structures, v. 20, n. 16, p. 1923-1939, 2009.

SARAVANOS, D. Mixed laminate theory and finite element for smart piezoelectric composite shell elements. AIAA Journal, v. 35, p. 1327-1333, 1997.

SETHIAN, J.; WIEGMANN, A. Structural boundary design via level set and immersed interface method. Journal of Computational Physics, v. 163, p. 489-528, 2000.

SHUYU, L. Analysis of the sandwich piezoelectric ultrasonic transducer in coupled vibration. Journal of Acoustical Society of America, v. 117, n. 2, p. 653-661, 2005.

SIGMUND, O. A 99 line topology optimization code written in MATLAB. Structural and Multidisciplinary Optimization, v. 21, n. 2, p. 120-127, 2001.

SIGMUND, O. Morphology-based black and white filters for topology optimization. Structural and Multidisciplinary Optimization, Springer, v. 33, n. 4, p. 401-424, 2007. 
SIGMUND, O.; PETERSSON, J. Numerical instabilities in topology optimization: A survey on procedures dealing with checkerboards, mesh-dependencies and local minima. Structural Optimization, v. 16, n. 1, p. 68-75, ago. 1998.

SIGMUND, O.; TORQUATO, S. Design of materials with extreme thermal expansion using a three-phase topology optimization method. Journal of the Mechanics and Physics of Solids, v. 45, p. 1037-67, 1997.

SILVA, E. C. N.; FONSECA, J. S. O.; KIKUCHI, N. Optimal design of piezoelectric microstructures. Computational Mechanics, v. 19, n. 3, p. 397-410, 1997.

SILVA, E. C. N.; KIKUCHI, N. Design of piezocomposite materials and piezoelectric transducers using topology optimization - part iii. Archives of Computational Methods in Engineering, v. 6, n. 4, p. 305-329, 1999.

SILVA, E. C. N.; KIKUCHI, N. Design of piezoelectric transducers using topology optimization. Smart Materials \& Structures, Iop Publishing Ltd, v. 8, n. 3, p. 350-364, 1999.

SILVA, E. C. N.; KÖGL, M. Design of piezoelectric bilaminar and c-block actuators using topology optimization. Smart Structures and Materials 2004: Modeling, Signal Processing, and Control, v. 5383, p. 265-276, 2004.

SILVA, E. C. N.; NISHIWAKI, S.; FONSECA, J. S. O.; KIKUCHI, N. Optimization methods applied to material and flextensional actuator design using the homogenization method. Computer Methods In Applied Mechanics and Engineering, v. 172, n. 1-4, p. 241-271, 1999.

SILVA, E. C. N.; NISHIWAKI, S.; KIKUCHI, N. Design of piezocomposite materials and piezoelectric transducers using topology optimization - part ii. Archives of Computational Methods in Engineering, v. 6, n. 3, p. 191-222, 1999.

SILVA, E. C. N.; NISHIWAKI, S.; KIKUCHI, N. Topology optimization design of flextensional actuators. Ieee Transactions On Ultrasonics Ferroelectrics and Frequency Control, v. 47, n. 3, p. 657-671, 2000.

SODANO, H. A.; INMAN, D. J. A review of power harvesting from vibration using piezoelectric materials. The Shock and Vibration Digest, v. 36, p. 197-205, 2004.

SODANO, H. A.; INMAN, D. J.; PARK, G. Comparison of piezoelectric energy harvesting devices for recharging batteries. Journal Of Intelligent Material Systems And Structures, Sage Publications Ltd, v. 16, n. 10, p. 799-807, 2005.

STEGMANN, J.; LUND, E. Discrete material optimization of general composite shell structures. International Journal For Numerical Methods In Engineering, v. 62, n. 14, p. 2009-2027, 2005.

STEGMANN, J.; LUND, E. Nonlinear topology optimization of layered shell structures. Structural and Multidisciplinary Optimization, v. 29, n. 5, p. 349-360, 2005.

STOLPE, M.; SVANBERG, K. An alternative interpolation scheme for minimum compliance topology optimization. Structural And Multidisciplinary Optimization, Springer-Verlag, v. 22, n. 2, p. 116-124, 2001. 
STUMP, F. Otimização topológica aplicada ao projeto de estruturas tradicionais e estruturas com gradação funcional sujeitas a restrição de tensão. Dissertação (Mestrado) — Escola Politécnica da USP, 2006.

SVANBERG, K. The method of moving asymptotes - a new method for structural optimization. International Journal fo Numerical Methods in Engineering, v. 24, p. 359-373, 1987.

SWAN, C. C.; KOSAKA, I. Voigt-Reuss topology optimization for structures with linear elastic material behaviours. International Journal for Numerical Methods in Engineering, v. 40, n. 16, p. 3033-3057, 1997.

TAKEZAWA, A.; NISHIWAKI, S.; KITAMURA, M. Shape and topology optimization based on the phase field method and sensitivity analysis. Journal of Computational Physics, v. 229, n. 7, p. 2697-2718, abr. 2010.

TIERSTEN, H. Hamilton's principle for linear piezoelectric media. Proceedings of the IEEE, v. 55, n. 8, p. 1523-1524, 1967.

TIMOSHENKO, S. Mecânica dos sólidos. [S.1.]: Ltc, 1984.

TZOU, H. S.; TSENG, C. I. Distributed piezoelectric sensor actuator design for dynamic measurement control of distributed parameter-systems - a piezoelectric finite-element approach. Journal Of Sound And Vibration, Academic Press Ltd, v. 138, n. 1, p. 17-34, 1990.

VANDERPLAATS, G. N. Numerical Optimization Techniques for Engineering Design: with Applications. [S.1.]: McGraw-Hill, New York, EUA, 1984.

WANG, S. Y.; TAI, K.; QUEK, S. T. Topology optimization of piezoelectric sensors/actuators for torsional vibration control of composite plates. Smart Materials and Structures, v. 15, p. 253-269, 2006.

WhitTingham, T. Medical diagnostic applications and sources. Progress in Biop, v. 93 , p. $84-110,2007$.

WILKIE, W.; INMAN, D.; LLOYD, J.; HIGH, J. Anisotropic laminar piezocomposite actuator incorporating machined pmn-pt single-crystal fibers. Journal of Intelligent Material Systems and Structures, Sage Publications, v. 17, n. 1, p. 15-28, 2006.

XIE, W.; CAO, C.; LÜ, Y.; HONG, Z.; WEI, B. Acoustic method for levitation of small living animals. Applied Physics Letters, v. 89, 2006.

XIE, Y.; STEVEN, G. A simple evolutionary procedure for structural optimization. Computers and Structures, v. 49, p. 885-896, 1993.

XIE, Y.; STEVEN, G. Optimal design of multiple load case structures using an evolutionary procedure. Engineering Computations, v. 11, p. 295-302, 1994.

YANG, R.; CHEN, C. Stress-based topology optimization. Structural and Multidisciplinary Optimization, Springer, v. 12, n. 2, p. 98-105, 1996. 
ZHENG, B.; CHANG, C.; GEA, H. Topology optimization of energy harvesting devices using piezoelectric materials. Structural And Multidisciplinary Optimization, v. 38, p. 17-23, 2009. 


\section{Apêndice A - MEF Piezelétrico}

\section{A.1 Definição do Sistema de Coordenadas Nodal-Local}

O sistema de coordenadas nodal-local é obtido considerando-se a orientação do sistema de coordenadas naturais (veja Fig. 2.3) e os eixos do sistema de coordenadas globais. Portanto, as funções de forma do elemento e as coordenadas globais de cada nó são utilizados. Assim, as coordenadas naturais nodais para $r$ e $s$ (utilizadas para calcular as funções de forma) são dadas na Tab. A.1.

Tabela A.1: Coordenadas naturais $r$ e $s$ para os nós de um elemento.

\begin{tabular}{c|rrrrrrrr}
\hline Nó $(n e)$ & 1 & 2 & 3 & 4 & 5 & 6 & 7 & 8 \\
\hline$r$ & 1 & -1 & -1 & 1 & 0 & -1 & 0 & 1 \\
$s$ & 1 & 1 & -1 & -1 & 1 & 0 & -1 & 0 \\
\hline
\end{tabular}

Utilizando esses pontos, dois vetores auxiliares podem ser calculados para cada nó $k$ do domínio:

$$
\begin{aligned}
& \mathbf{v}_{1 k}=\left\{\sum_{n e=1}^{q} x_{n e} \frac{\partial N_{n e}}{\partial r} \sum_{n e=1}^{q} y_{n e} \frac{\partial N_{n e}}{\partial r} \sum_{n e=1}^{q} z_{n e} \frac{\partial N_{n e}}{\partial r}\right\}^{T} \\
& \mathbf{v}_{2 k}=\left\{\sum_{n e=1}^{q} x_{n e} \frac{\partial N_{n e}}{\partial s} \sum_{n e=1}^{q} y_{n e} \frac{\partial N_{n e}}{\partial s} \sum_{n e=1}^{q} z_{n e} \frac{\partial N_{n e}}{\partial s}\right\}^{T}
\end{aligned}
$$

onde $N_{n e}$ e $x_{n e}$ são as funções de forma e as coordenadas globais $x$ do nó ne, respectivamente, e $q$ é o número de nós em cada elemento ( $q=8$ neste trabalho). Ou seja, para cada nó $k$ do domínio, todos os nós ne dos elementos à que $k$ pertence devem ser considerados para calcular seu sistema de coordenada nodal.

Assim, o vetor normal à superfície média no nó $k$ pode ser calculado por:

$$
\mathbf{V}_{n}^{k}=\frac{\mathbf{v}_{1 k} \times \mathbf{v}_{2 k}}{\left\|\mathbf{v}_{1 k} \times \mathbf{v}_{2 k}\right\|}
$$


Entretanto, se um nó $k$ pertence a mais de um elemento, a Eq. (A.2) deve ser calculada para cada elemento ao redor de $k$, e então, eles devem ser somados e normalizados pela seguinte expressão:

$$
\mathbf{V}_{n}^{k}=\frac{\sum_{e=1}^{e_{n}} \mathbf{V}_{n}^{k_{e}}}{\left\|\sum_{e=1}^{e_{n}} \mathbf{V}_{n}^{k_{e}}\right\|}
$$

onde $e_{n}$ é o número de elementos aos quais o nó $k$ pertence.

Utilizando $\mathbf{V}_{n}^{k}$ e os eixos do sistema de coordenada global, os vetores unitários tangentes à superfície média no nó $k$ podem ser calculados por:

$$
\begin{aligned}
\mathbf{V}_{1}^{k} & =\frac{\mathbf{e}_{y} \times \mathbf{V}_{n}^{k}}{\left\|\mathbf{e}_{y} \times \mathbf{V}_{n}^{k}\right\|} \\
\mathbf{V}_{2}^{k} & =\mathbf{V}_{n}^{k} \times \mathbf{V}_{1}^{k}
\end{aligned}
$$

onde $\mathbf{e}_{y}=\left[\begin{array}{lll}0 & 1 & 0\end{array}\right]$. Se $\mathbf{V}_{n}^{k}$ é paralelo à $\mathbf{e}_{y}$, então $\mathbf{V}_{1}^{k}=\mathbf{e}_{z}$.

\section{A.2 Obtenção da matriz elástica $\mathrm{C}$ para materiais isotrópicos e ortotrópicos}

Para obter a matriz elástica na forma

$$
\mathbf{c}^{E}=\left[\begin{array}{cccccc}
c_{11} & c_{12} & c_{13} & 0 & 0 & 0 \\
c_{12} & c_{22} & c_{23} & 0 & 0 & 0 \\
c_{13} & c_{23} & c_{33} & 0 & 0 & 0 \\
0 & 0 & 0 & c_{66} & 0 & 0 \\
0 & 0 & 0 & 0 & c_{44} & 0 \\
0 & 0 & 0 & 0 & 0 & c_{55}
\end{array}\right]
$$


a partir dos módulos de Young $\left(E_{i}\right)$ e coeficientes de Poisson $\left(\nu_{i j}\right)$ de materiais isotrópicos e ortotrópicos, é necessário inverter a seguinte matriz

$$
\overline{\mathbf{c}}=\left[\begin{array}{cccccc}
\frac{1}{E_{1}} & -\frac{\nu_{21}}{E_{2}} & -\frac{\nu_{31}}{E_{3}} & 0 & 0 & 0 \\
-\frac{\nu_{12}}{E_{1}} & \frac{1}{E_{2}} & -\frac{\nu_{32}}{E_{3}} & 0 & 0 & 0 \\
-\frac{\nu_{13}}{E_{1}} & -\frac{\nu_{23}}{E_{2}} & \frac{1}{E_{3}} & 0 & 0 & 0 \\
0 & 0 & 0 & \frac{1}{G_{12}} & 0 & 0 \\
0 & 0 & 0 & 0 & \frac{1}{G_{23}} & 0 \\
0 & 0 & 0 & 0 & 0 & \frac{1}{G_{31}}
\end{array}\right]
$$

onde $G_{i j}=E_{i} / 2\left(1+\nu_{i j}\right)$ e $\frac{\nu_{i j}}{E_{i}}=\frac{\nu_{j i}}{E_{j}}$

Para materiais isotrópicos, os valores de $E, \nu$ e $G$ são iguais para todas as direções. Já para materiais ortotrópicos, tem-se $E_{3}=E_{2}, \nu_{31}=\nu_{21}$. Assim:

$$
\mathbf{c}^{E}=\overline{\mathbf{c}}^{-1}
$$




\section{Apêndice B - Validação do MEF Implementado}

Para que o MOT tenha um bom desempenho na obtenção de topologias ótimas, é muito importante que as análises feitas pelo MEF estejam corretas e precisas. Assim é necessário validar o MEF implementado comparando-o com análises feitas por algum método analítico, por algum software comercial de elementos finitos ou por análises apresentadas em artigos na literatura utilizando elementos já bem fundamentados.

Nesta seção é apresentada a validação do MEF de casca multi-camada piezocompósita utilizada neste trabalho. A validação é feita comparando-se resultados de análises utilizando o MEF implementado com resultados de análises apresentados por Kögl e Bucalem (2005a) e por Balamurugan e Narayanan (2008), e com o software comercial Ansys.

São apresentados 5 exemplos para validar o MEF implementado:

1.atuador bilaminar plano somente com material piezelétrico;

2.sensor de placa com uma camada de material piezelétrico;

3.atuador de casca piezocompósito, com material piezelétrico e ortotrópico;

4.coletor de energia em regime harmônico com circuito elétrico acoplado;

5.travamento por cisalhamento.

Nos 4 primeiros itens, são avaliados os valores de deslocamentos ou de potencial elétrico gerado pelo material piezelétrico. Já no item 5 é feita uma verificação se o elemento implementado apresenta ou não o problema de travamento por cisalhamento ("shear locking").

O elemento de casca piezocompósito implementado passa a ser representado pela sigla PCS8 ("PiezoComposite Shell" com 8 nós) de modo a facilitar sua identificação nas comparações das seções a seguir. 


\section{B.1 Atuador Bilaminar Plano}

Neste primeiro exemplo, é avaliada uma viga bilaminar piezelétrica plana representada na Fig. B.1.

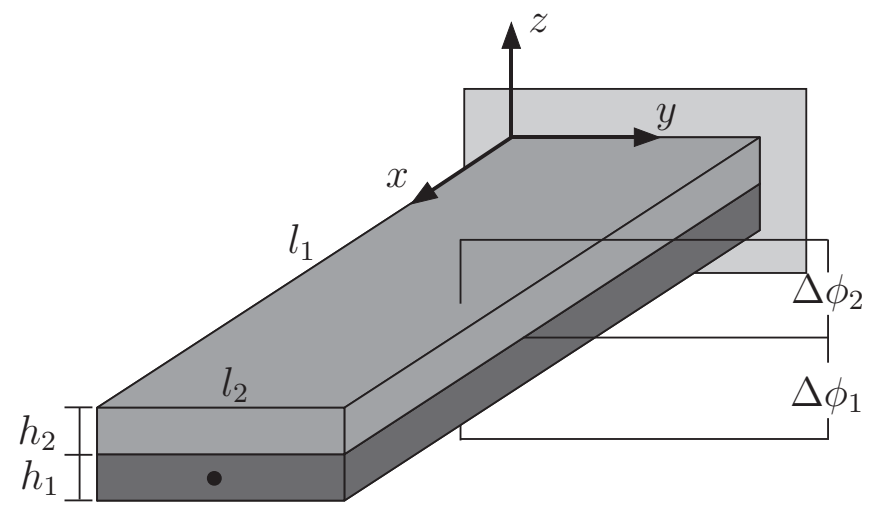

A

Figura B.1: Modelo da viga bilaminar piezelétrica.

Este é um exemplo clássico de atuador bilaminar que consiste em duas camadas piezelétricas (propriedades do PZT podem ser encontradas na Tab. C.3 do Apêndice C) com espessuras $h_{1}$ e $h_{2}$, atuadas pelos potenciais elétricos $\Delta \phi_{1}$ e $\Delta \phi_{2}$, respectivamente. A viga tem dimensões iguais a $l_{1}=100 \mathrm{~mm} \mathrm{e} l_{2}=20 \mathrm{~mm}$, e é engastada em $x=0$. São aplicados campos elétricos invertidos em cada camada, pois, enquanto uma camada estica, a outra contrai, provocando um movimento de flexão na viga. Os campos elétricos são iguais a $E_{1}=-E_{2}=-10 \mathrm{~V} / \mathrm{mm}$. Considerando que a polarização é definida ao longo da espessura no sentido positivo de $z$ na Fig. B.1 e que o campo elétrico pode ser calculado como $E=-\Delta \phi / h$, é possível obter os valores de potenciais elétricos aplicados em cada camada por $\Delta \phi=-E h$. Neste exemplo, a espessura da camada 1 é fixa e tem valor igual a $h_{1}=1 \mathrm{~mm}$ e portanto, $\Delta \phi_{1}=10 \mathrm{~V}$. Já $h_{2}$ é variável com valores iguais a 10, 1 e $0,1 \mathrm{~mm}$ resultando em $\Delta \phi_{2}$ igual a $-100,-10$ e $-1 \mathrm{~V}$, respectivamente. A Tab. B.1 mostra os deslocamentos verticais no ponto A para os 3 casos de espessuras $h_{2}$, obtidos utilizando o elemento PCS8 e comparando-o com os resultados apresentados por Kögl e Bucalem (2005a), que utiliza o elemento MITC9, e Balamurugan e Narayanan (2008), que utiliza um elemento piezelétrico de casca degenerado de 9 nós, representado aqui por PLS9.

Analisando a Tab. B.1 nota-se que os resultados obtidos para o elemento PCS8 apresentam excelente concordância com os resultados apresentados por Kögl e Bucalem (2005a) e Balamurugan e Narayanan (2008), sendo que a diferença entre os resultados apresentados é inferior a $6 \%$. Assim, por apresentar valores baixos de diferenças entre 


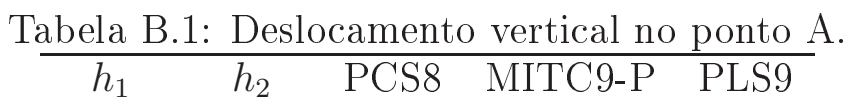

\begin{tabular}{ccccc}
$(\mathrm{mm})$ & $(\mathrm{mm})$ & $(\mu \mathrm{m})$ & $(\mu \mathrm{m})$ & $(\mu \mathrm{m})$ \\
\hline 1 & 10 & 0,78 & 0,78 & 0,76 \\
1 & 1 & 15,31 & 15,39 & 15,29 \\
1 & 0,1 & 7,91 & 7,94 & 7,88 \\
\hline
\end{tabular}

outros modelos, pode-se concluir que o elemento implementado apresenta excelente desempenho na obtenção do campo de deslocamentos no caso de um atuador bilaminar.

\section{B.2 Sensor de Placa Piezelétrico}

No segundo exemplo utilizado para validar o MEF implementado, é avaliado o desempenho de um sensor piezelétrico multi-camada de placa. O modelo consiste em uma placa metálica quadrada de lados $l_{a}=100 \mathrm{~mm}$ e espessura igual a $h_{a}=1 \mathrm{~mm}$ no qual são acoplados quatro sensores piezelétricos quadrados de lados $l_{p}=25 \mathrm{~mm}$ e espessura igual a $h_{p}=0,1 \mathrm{~mm}$. Uma representação deste modelo pode ser visto na Fig. B.2, a qual mostra também o posicionamento dos sensores sobre a placa. A estrutura está engastada em $x=0$ e uma força distribuída igual a $t=0.1 \mathrm{~N} / \mathrm{mm}$ é aplicada ao longo de metade do lado $y=0$, como mostra a Fig. B.2. Os materiais utilizados são o alumínio e o PZT (KÖGL; BUCALEM, 2005a) e as propriedades desses materiais podem ser encontradas, respectivamente, nas Tabs. C.1 e C.3 no Apêndice C.

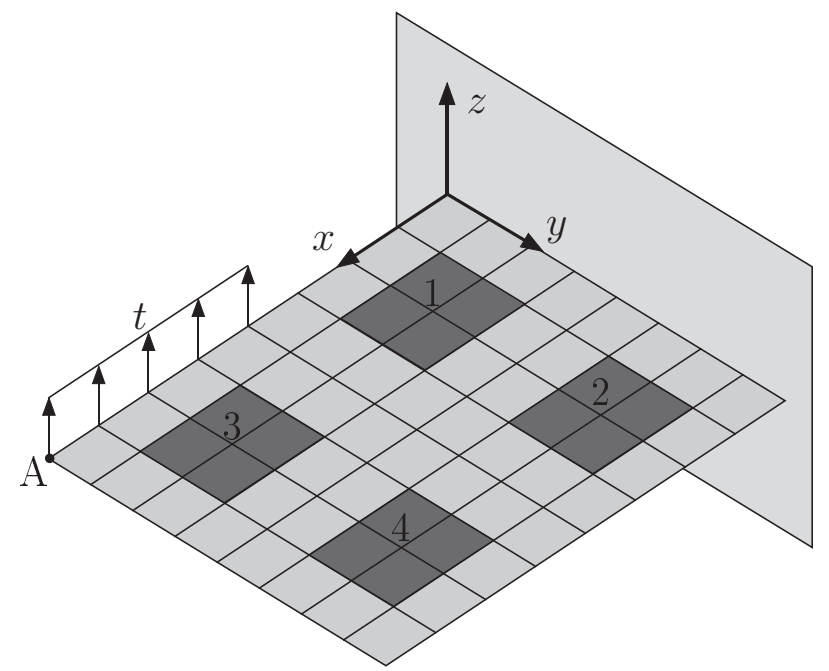

Figura B.2: Modelo de um sensor de placa piezelétrico.

Os potenciais elétricos $\Delta \phi_{1}$ a $\Delta \phi_{4}$ (gerados nos sensores 1 a 4 respectivamente) e o deslocamento vertical $u_{z}^{A}$ no ponto A são apresentados na Tab. B.2. Os resultados obtidos 
Tabela B.2: Potenciais elétricos $\Delta \phi_{1}$ a $\Delta \phi_{4}$ [V] e deslocamento $u_{z}^{A}$ [mm].

\begin{tabular}{lrccccc}
\hline Elemento & Malha & $\Delta \phi_{1}$ & $\Delta \phi_{2}$ & $\Delta \phi_{3}$ & $\Delta \phi_{4}$ & $u_{z}^{A}$ \\
\hline MITC4-P & $8 \times 8$ & 16,879 & 9,751 & 2,912 & 4,305 & 2,247 \\
MITC4-P & $64 \times 64$ & 16,483 & 9,570 & 2,881 & 4,172 & 2,263 \\
MITC9-P & $8 \times 8$ & 16,608 & 9,643 & 2,902 & 4,205 & 2,263 \\
MITC9-P & $24 \times 24$ & 16,391 & 9,508 & 2,887 & 4,140 & 2,267 \\
HEX20-P & $24 \times 24 \times 4$ & 16,654 & 9,667 & 2,908 & 4,215 & 2,261 \\
PCS8 & $8 \times 8$ & 16,898 & 9,879 & 3,144 & 4,276 & 2,268 \\
PCS8 & $24 \times 24$ & 16,521 & 9,594 & 2,968 & 4,182 & 2,268 \\
PCS8 & $64 \times 64$ & 16,473 & 9,559 & 2,921 & 4,164 & 2,268 \\
\hline
\end{tabular}

utilizando o elemento implementado PCS8 são comparados com os dados apresentados por Kögl e Bucalem (2005a).

Analisando a Tab. B.2, conclui-se que o elemento implementado PCS8 utilizado como sensor está em excelente concordância com os elementos MITC4-P, MITC9-P e HEX20-P apresentados por Kögl e Bucalem (2005a).

\section{B.3 Atuador de Casca Piezocompósito}

O próximo exemplo consiste numa estrutura de casca na forma de $1 / 4$ de cilindro, composta por quatro camadas de material ortotrópico e uma camada de material piezelétrico, conforme representado na Fig. B.3. A estrutura é engastada nas duas bordas $z=0$ e $y=0$ somente da camada piezelétrica. Duas diferentes configurações de empilhamento de camadas são consideradas, [0/90/90/0/p] e $[p / 0 / 90 / 90 / 0]$, onde $p$ representa a camada piezelétrica e os valores 0 e 90 representam o ângulo de orientação das fibras do material ortotrópico, cujas direções estão representadas na Fig. B.3.

O raio $R=200 \mathrm{~mm}$ é considerado na linha média da camada mais interna e o comprimento é igual a $l=R \pi / 2 \mathrm{~mm}$. Cada camada ortotrópica tem espessura igual a $h_{c}=0.375 \mathrm{~mm}$ e a camada piezelétrica tem espessura igual a $h_{p}=0,5 \mathrm{~mm}$, totalizando $h=2 \mathrm{~mm}$. Neste exemplo, é feita a validação para o caso de atuadores curvos, medindo o deslocamento radial na linha $z=l / 2$ decorrente da aplicação de um potencial elétrico igual a $\Delta \phi=200 \mathrm{~V}$ na camada piezelétrica. Assim, além de se considerar a estrutura curva de casca, é avaliado também o comportamento da orientação das fibras da camada de material ortotrópico, ou seja, verifica-se a influência da orientação das fibras no deslocamento gerado pela atuação do material piezelétrico.

As propriedades do material piezelétrico podem ser encontradas na Tab. C.4. Para 


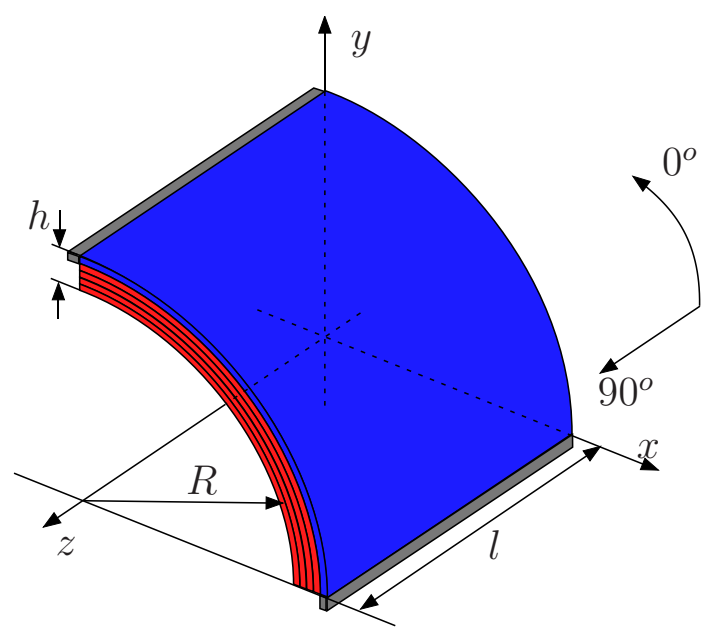

(a) $0 / 90 / 90 / 0 / p$

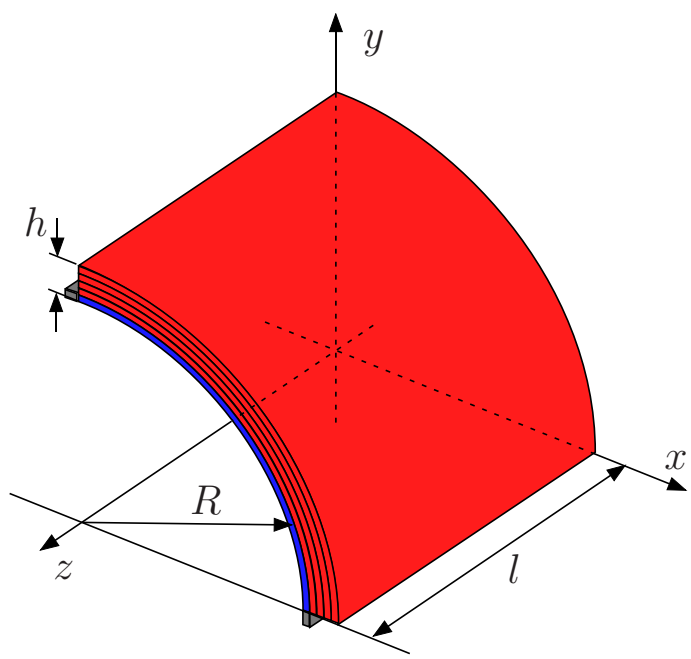

(b) $p / 0 / 90 / 90 / 0$

Figura B.3: Modelo de um atuador de casca piezocompósito.

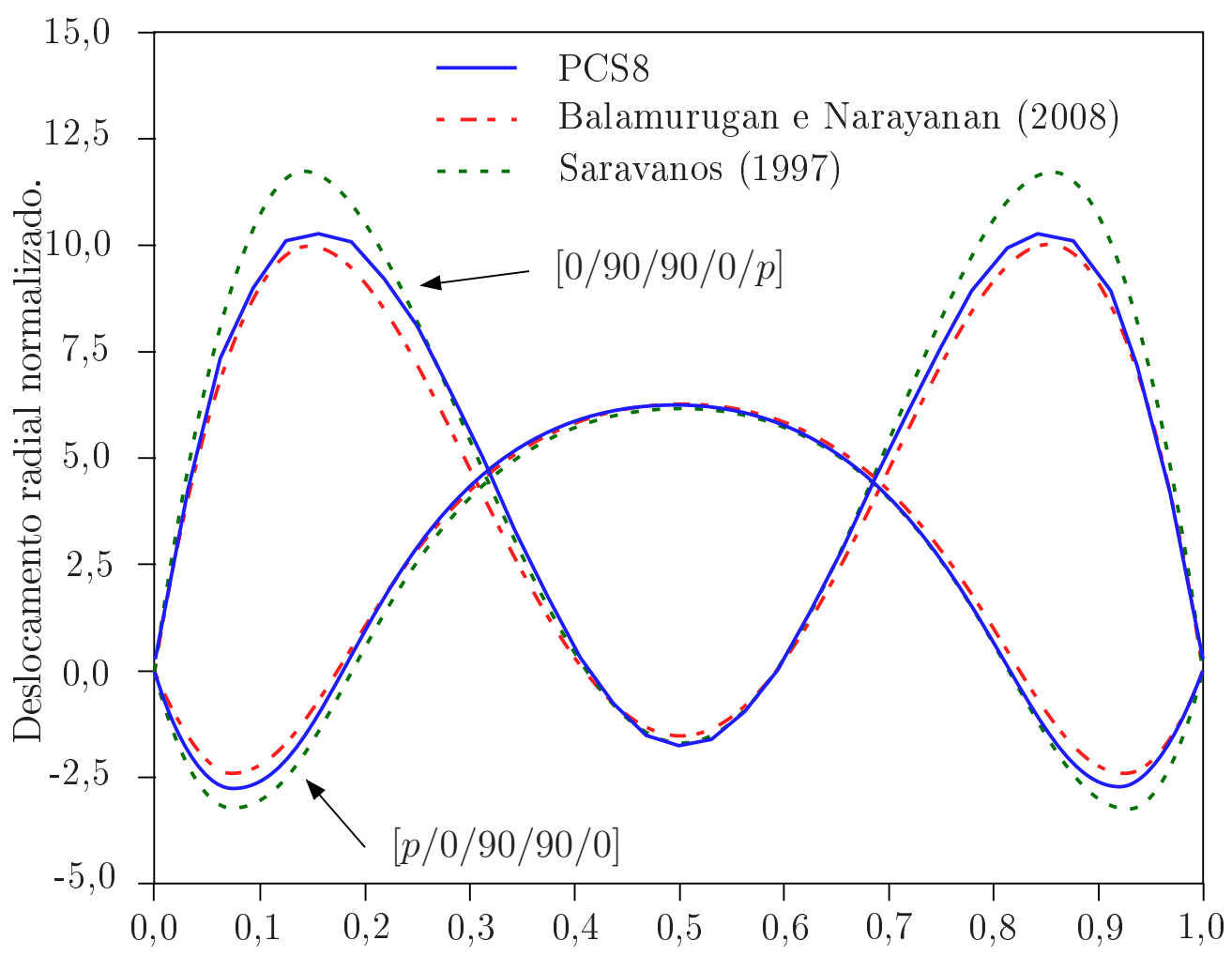

Distância circunferencial normalizada.

Figura B.4: Deslocamento radial normalizado $w / h$ ao longo da linha média em $z=l / e$ da primeira camada.

o material ortotrópico, é utilizado a fibra de carbono com matriz de epóxi, cujas propriedades podem ser encontradas na Tab. C.5.

Na Fig. B.4 é mostrado o perfil dos deslocamentos radiais ao longo da linha média 
da primeira camada para $z=l / 2$. A linha azul contínua representa o resultado obtido utilizando o elemento PCS8, enquanto as linhas tracejadas vermelha e verde representam os resultados obtidos por Balamurugan e Narayanan (2008) e Saravanos (1997), respectivamente. Analisando os gráficos da Fig. B.4, nota-se que os resultados obtidos utilizando o elemento implementado e os da literatura são bem próximos, validando o MEF de casca piezocompósito multi-camadas.

\section{B.4 Coletor de Energia em Regime Harmônico com Circuito Elétrico Acoplado}

Nesta seção é apresentada a validação do MEF implementado para coletores de energia operando em regime harmônico com excitação de base, contendo um circuito elétrico (resistor) acoplado nos eletrodos da cerâmica piezelétrica. O MEF implementado é comparado com a modelagem feita no software comercial Ansys. Entretanto, como o ANSYS não possui elemento de casca piezelétrico, a comparação é feita com o elemento sólido SOLID5 que possui graus de liberdade mecânicos e elétricos.

Na Fig. B.5 é apresentada uma representação da geometria do domínio utilizado neste exemplo, o qual consiste numa estrutura de $1 / 4$ de cilindro com dimensões $l_{1}=0,05 \mathrm{~mm}$, $r=0,1 \mathrm{~mm}, h_{1}=1 \mathrm{~mm}$ e $h_{2}=0,5 \mathrm{~mm}$. O raio $r$ é medido do centro do cilindro até a linha média da primeira camada. A estrutura é engastada numa base (representada pelo bloco cinza na Fig. B.5) que vibra somente na direção $z$ com amplitude de $1 \mathrm{~mm}$.

Os materiais utilizados são: alumínio e PZT para as camadas interna (vermelha) e externa (azul), respectivamente. As propriedades desses materiais podem ser encontrados nas Tabs. C.1 e C.3, respectivamente.

Neste caso, é feita uma análise harmônica (resposta em frequência) entre 0 e $2000 \mathrm{~Hz}$ com passo de $10 \mathrm{~Hz}$ (faixa de frequência aleatória somente para verificação) com amortecimento estrutural $\beta=10^{-5}$ e com um resistor de $R=1000 \Omega$ acoplado aos eletrodos do material piezelétrico.

A Fig. B.6 mostra o gráfico da resposta em frequência das duas análises, a linha azul representando o elemento implementado PCS8 e a linha vermelha, o elemento SOLID5 do Ansys. Nesse gráfico é possível observar que os valores de potência elétrica obtidos utilizando o PCS8 estão bem próximos com os valores obtidos com o elemento SOLID5 do Ansys. Entretanto, próximo às frequências de ressonância de $180 \mathrm{~Hz}$ e $560 \mathrm{~Hz}$, existe uma diferença bastante significativa entre os valores obtidos nos modelos, que pode 


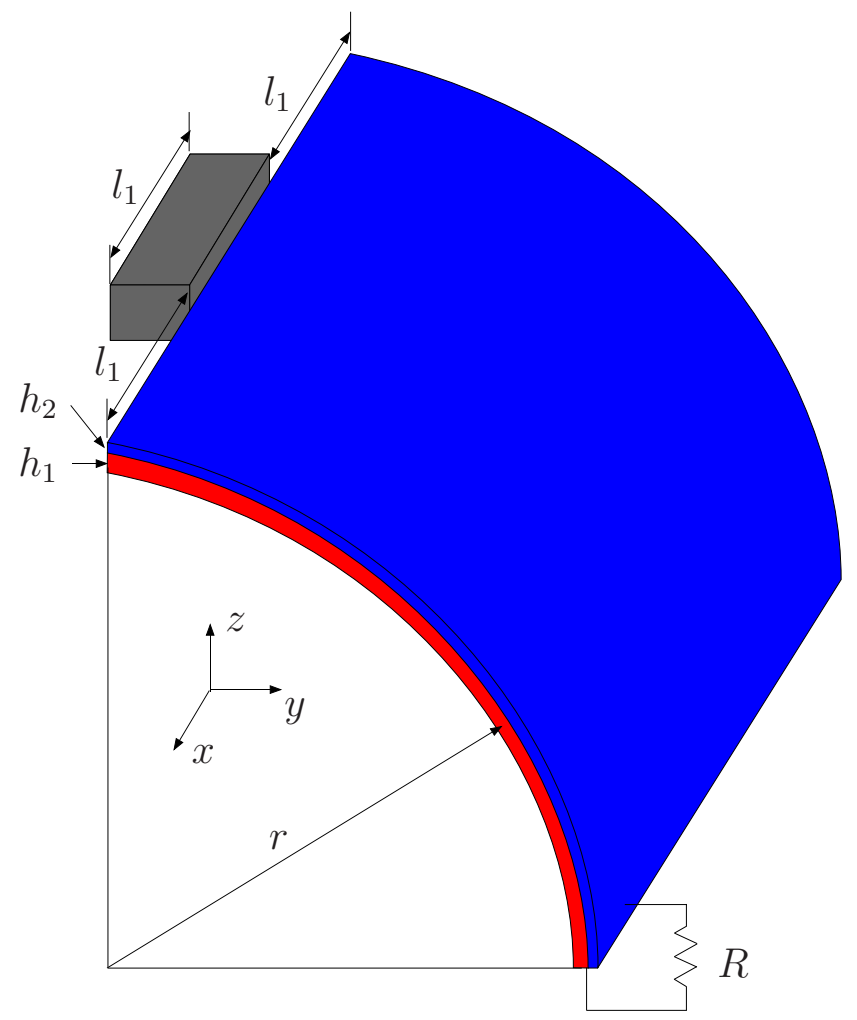

Figura B.5: Modelo utilizado para verificação dos coletores de energia.

ter sido causada pela diferença do algorítimo utilizado pelo Matlab e pelo Ansys para resolver sistemas lineares, pela diferença do tipo de elemento utilizado ou pela própria singularidade causada no sistema de equações de equilíbrio quando chega próximo à ressonância. Contudo, essa diferença entre valores na ressonância não invalida elemento PCS8.

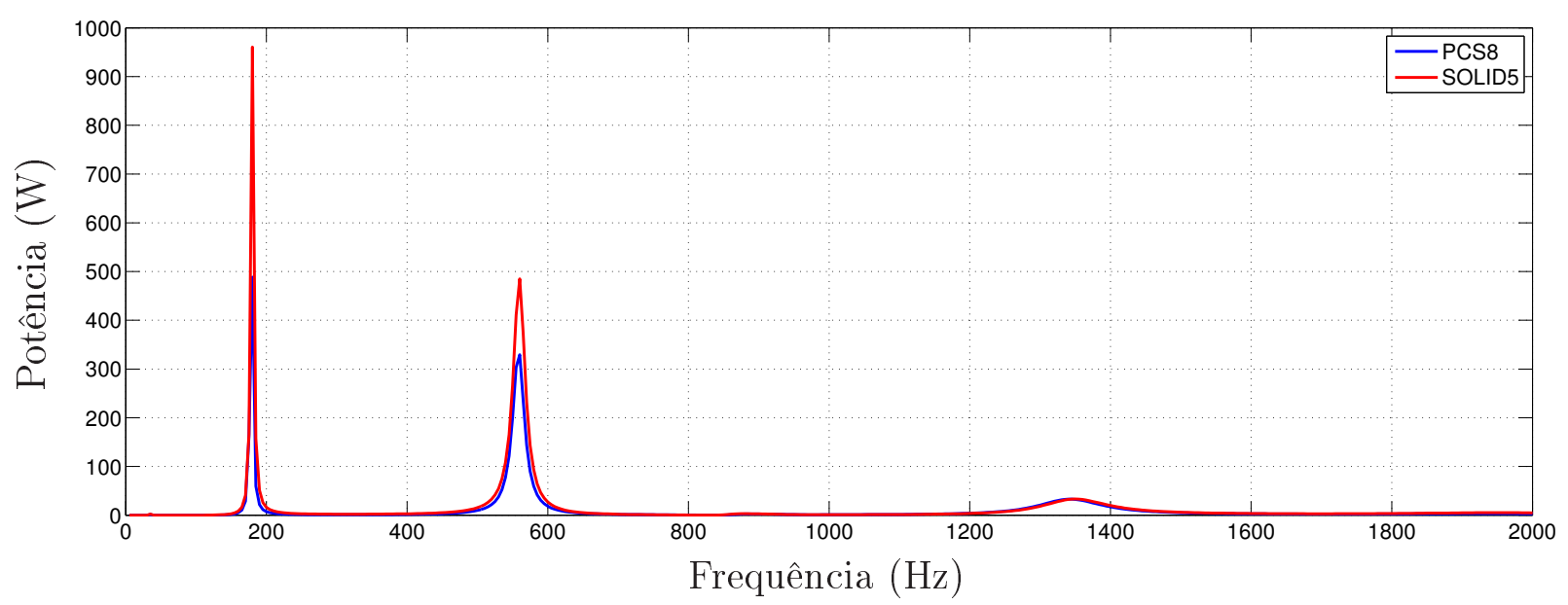

Figura B.6: Gráfico da resposta em frequência do modelo apresentado entre 0 e 2000 Hz.

No gráfico da Fig. B.7 é apresentada novamente a resposta em frequência para a mesma faixa de 0 a $2000 \mathrm{~Hz}$, com a diferença que o valor no eixo das ordenadas representa o 
logaritmo na base 10 da potência elétrica. Assim, é possível observar com mais precisão os valores das frequências de ressonância nessa faixa de frequência analisada. Desse gráfico, nota-se que os valores de frequência de ressonância obtidos pelo PCS8 está praticamente igual aos valores obtidos utilizando o SOLID5 do Ansys.

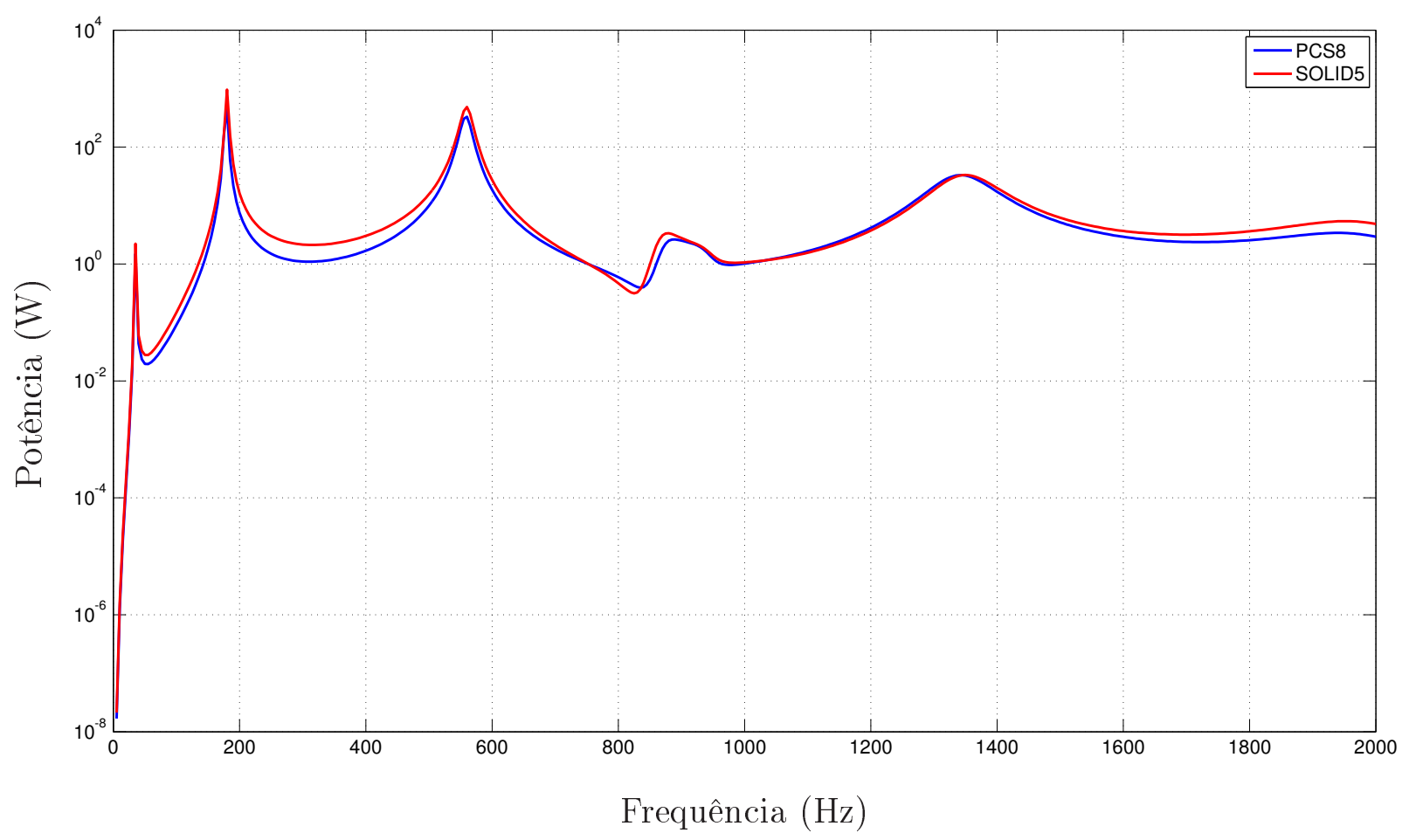

Figura B.7: Gráfico logarítmico da potência elétrica em função da frequência do modelo apresentado entre 0 e $2000 \mathrm{~Hz}$.

Já as Figs. B.8 e B.9 apresentam a resposta em frequência de duas faixas de frequência (400-700 Hz e 1200-1500 Hz) com maior precisão (passo de 1Hz) para avaliar melhor os valores de potência obtidos. A Fig. B.8 mostra o gráfico próximo à frequência de ressonância de $560 \mathrm{~Hz}$ que apresenta uma diferença significativa entre os valores obtidos pelos modelos, chegando a 30\% no ponto máximo da ressonância, porém na região estável do gráfico, essa diferença não passa de 5\%. Já a Fig. B.9 apresenta o gráfico próximo da frequência de $1350 \mathrm{~Hz}$ que, apesar dos valores estarem praticamente iguais, existe uma diferença no valor da frequência de ressonância, causando uma defasagem em todo o gráfico do PCS8. Entretanto, essa diferença é menor que 2\%, o que não prejudica na validação do MEF. 


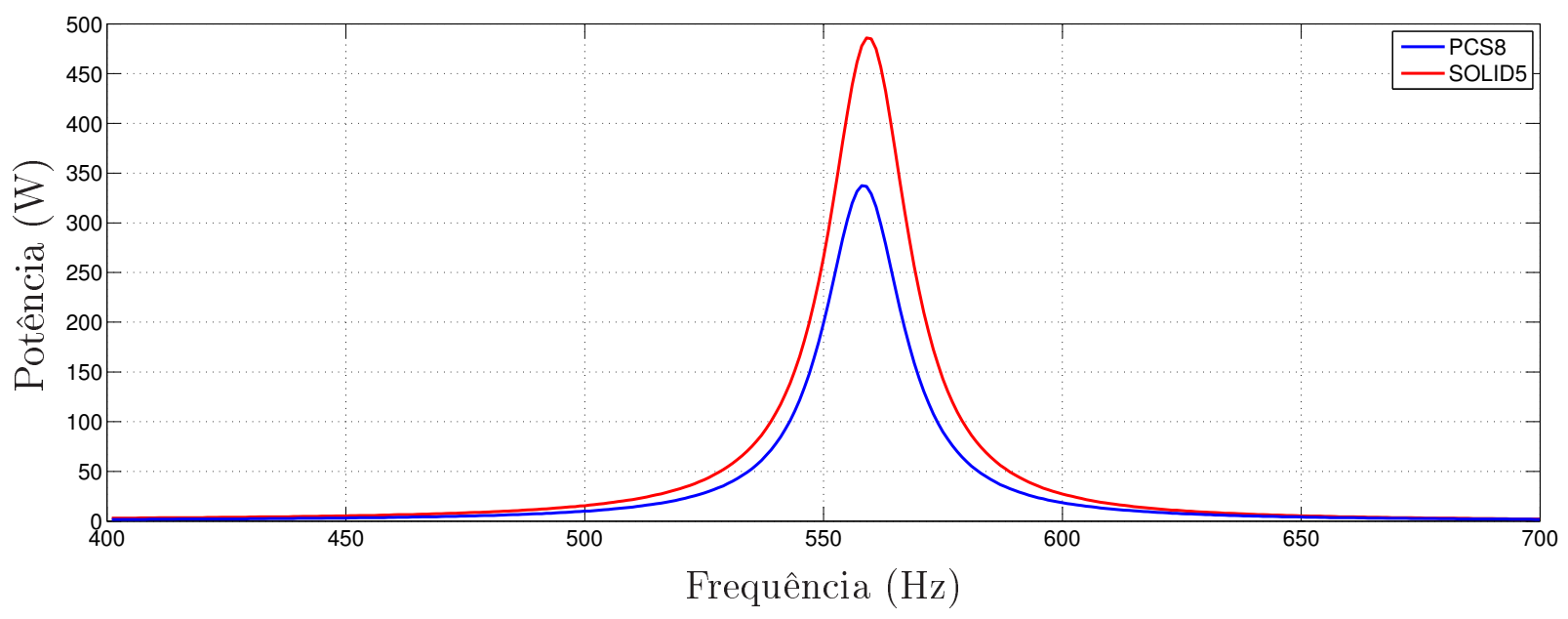

Figura B.8: Gráfico da resposta em frequência do modelo apresentado entre 400 e $700 \mathrm{~Hz}$ com intervalo de $1 \mathrm{~Hz}$.

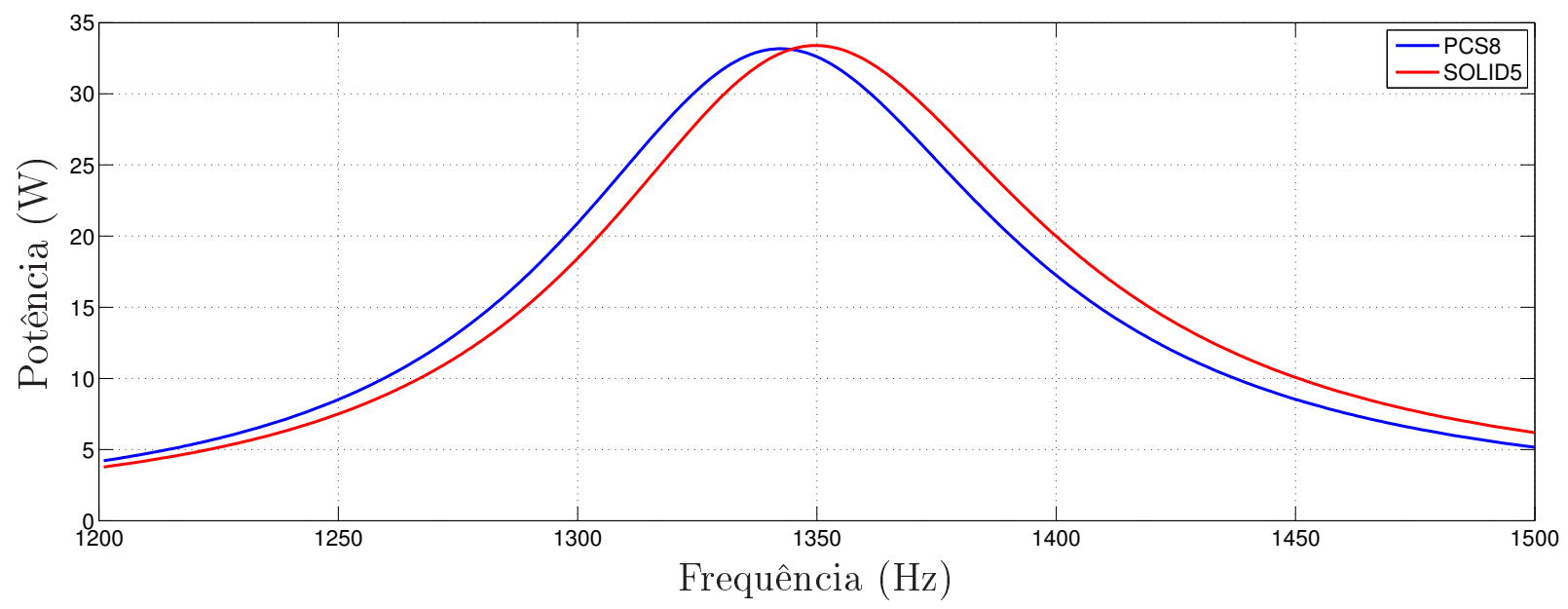

Figura B.9: Gráfico da resposta em frequência do modelo apresentado entre 1200 e 1500 $\mathrm{Hz}$ com intervalo de $1 \mathrm{~Hz}$.

\section{B.5 Travamento por Cisalhamento}

Dependendo do tipo de elementos de placa e casca utilizados no MEF, o modelo pode estar sujeito ao efeito chamado de travamento por cisalhamento (ou "shear locking") quando a razão de aspecto da estrutura (razão entre comprimento e espessura) for muito grande (BATHE, 1996; KÖGL; BUCALEM, 2005b, 2005a). O "shear locking" faz com que estruturas muito finas tenham rigidez artificial muito grande, comprometendo a solução da análise da estrutura. Assim, se não houver um tratamento para evitar o "shear locking", todo o procedimento de otimização topológica pode ser invalidado.

Neste trabalho, é implementado o elemento de casca piezocompósito de 8 nós. Para evitar o "shear locking", é utilizada a integração reduzida seletiva, ou seja, na integração numérica para obtenção das matrizes de rigidez, são utilizados 9 pontos de Gauss para os 
efeitos de membrana e flexão e 4 pontos para os efeitos de cisalhamento. Para verificar se este procedimento está ou não sujeito aos efeitos do "shear locking", é utilizada a mesma abordagem utilizada por Kögl e Bucalem (2005a). Neste trabalho, os autores consideram uma placa quadrada metálica (Aço) com um sensor piezelétrico acoplado em sua superfície superior. Os autores afirmam que, quanto menor for a espessura do sensor piezelétrico, menor deverá ser a sua influência na rigidez total da estrutura. Os autores ainda mostram que, se nenhuma técnica for utilizada para prevenir o "shear locking", diferenças maiores que $16 \%$ podem ser encontradas no campo de deslocamentos da estrutura.

Considere uma placa de aço quadrada de lado igual a $l=1000 \mathrm{~mm}$ e espessura igual a $h_{0}$. Uma camada piezelétrica de tamanho $1000 \mathrm{~mm} \times 200 \mathrm{~mm}$ e espessura igual a $h_{1}$ é colada na superfície superior da placa de aço conforme apresentado na Fig. B.10. A estrutura é engastada em $x=0$ e uma força distribuída $t_{z}=1 \mathrm{~N} / \mathrm{m}$ é aplicada em $x=l$. Os cálculos são feitos para uma malha de $10 \times 10$ elementos na camada de aço e $10 \times 2$ elementos na camada de PZT. As propriedades do aço e do PZT utilizados podem ser conferidos nas Tabs. C.2 e C.3, respectivamente.

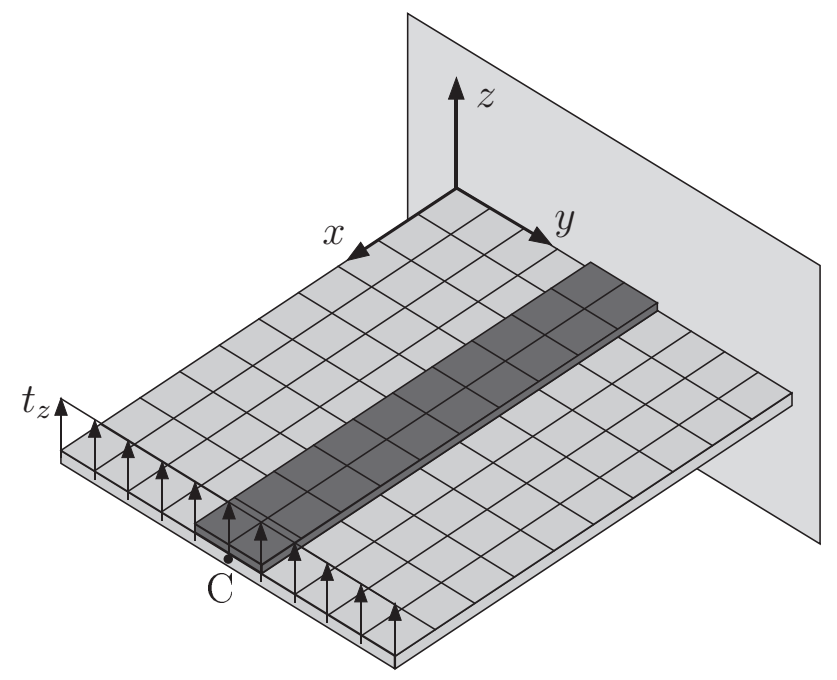

Figura B.10: Modelo utilizado para verificação do efeito de "shear locking".

Os resultados para o deslocamento vertical $u_{z}^{C}$ no ponto C (vide Fig. B.10) são obtidas para diferentes espessuras $h_{0}$ e $h_{1}$. Na Tab. B.3 são apresentados os valores da razão $u_{z}^{C} / u_{z}^{C, r e f}$, onde $u_{z}^{C, r e f}$ é o deslocamento vertical no ponto $\mathrm{C}$ sem a camada piezelétrica. Assim, quanto menor for a espessura $h_{1}$, a razão $u_{z}^{C} / u_{z}^{C, r e f}$ deve convergir para 1 . Os dados para os elementos MITC e isoparamétrico foram obtidos do trabalho de Kögl e Bucalem (2005a).

Analisando a Tab. B.3, nota-se que os resultados utilizando o elemento implementado PCS8 apresenta resultados idênticos ao do elemento MITC apresentado por Kögl e 
Tabela B.3: Efeito do travamento para finas camadas de material piezelétrico.

\begin{tabular}{lllllll}
\hline$h_{0}[\mathrm{~mm}]$ & 1 & & \multicolumn{1}{c}{10} \\
\hline$h_{1} / h_{0}$ & PCS8 & MITC & isop. & PCS8 & MITC & isop. \\
\hline 0,1 & 0,976 & 0,976 & 0,077 & 0,976 & 0,976 & 0,819 \\
0,01 & 0,998 & 0,998 & 0,363 & 0,998 & 0,998 & 0,978 \\
0,001 & 1,000 & 0,999 & 0,836 & 1,000 & 1,000 & 0,998 \\
\hline
\end{tabular}

Bucalem (2005a). Como era esperado, conforme a espessura da camada piezelétrica diminui, sua rigidez se torna desprezível com relação à rigidez total da estrutura. Entretanto, os resultados utilizando o elemento isoparamétrico sem tratamento do "shear locking" (apresentado por Kögl e Bucalem (2005a)) são fortemente afetados pela camada piezelétrica quando $h_{0}=1 \mathrm{~mm}$.

\section{B.6 Conclusão}

A partir dos exemplos apresentados anteriormente, pode-se concluir que o elemento implementado neste trabalho apresenta ótimo desempenho na modelagem de estruturas finas de casca e placa multi-camadas, considerando atuação, sensoriamento e coleta de energia, operando nos regime estático e dinâmico amortecido, e com a possibilidade de se utilizar materiais ortotrópicos de forma a modificar a rigidez da estrutura conforme o ângulo da orientação das fibras, sem apresentar travamento por cisalhamento. 


\section{Apêndice C - Propriedade dos Materiais}

Tabela C.1: Propriedades do Alumínio

$E=71 \mathrm{GPa} \quad \nu=0,334 \quad \rho=2700 \mathrm{~kg} / \mathrm{m}^{3} \quad \sigma_{y}=300 \mathrm{MPa}$

Tabela C.2: Propriedades do Aço

$E=210 \mathrm{GPa} \quad \nu=0,3$

Tabela C.3: Propriedades do material piezelétrico utilizado por Kögl e Bucalem (2005a).

\begin{tabular}{rrrrr}
\hline$C_{11}=$ & $107,60 \mathrm{GPa}$ & $e_{31}=$ & $-9,6 \mathrm{C} / \mathrm{m}^{2}$ & $\rho=7800 \mathrm{~kg} / \mathrm{m}^{3}$ \\
$C_{12}=$ & $63,12 \mathrm{GPa}$ & $e_{33}=$ & $15,1 \mathrm{C} / \mathrm{m}^{2}$ & $\sigma_{d}=50 \mathrm{MPa}$ \\
$C_{13}=$ & $63,85 \mathrm{GPa}$ & $e_{15}=$ & $12,0 \mathrm{C} / \mathrm{m}^{2}$ & \\
$C_{33}=$ & $100,40 \mathrm{GPa}$ & $\epsilon_{11}=$ & $1936 \epsilon_{0} \mathrm{~F} / \mathrm{m}$ & \\
$C_{44}=$ & $19,62 \mathrm{GPa}$ & $\epsilon_{33}=$ & $2109 \epsilon_{0} \mathrm{~F} / \mathrm{m}$ & \\
$C_{66}=$ & $22,24 \mathrm{GPa}$ & $\epsilon_{0}=$ & $8,854 \times 10^{-12}$ & \\
\hline
\end{tabular}

Tabela C.4: Propriedades do material piezelétrico utilizado por Balamurugan e Narayanan (2008).

\begin{tabular}{rrrrrr}
\hline$C_{11}=$ & $139,00 \mathrm{GPa}$ & $e_{31}=$ & $-14,8 \mathrm{C} / \mathrm{m}^{2}$ & $\rho=$ & $7500 \mathrm{~kg} / \mathrm{m}^{3}$ \\
$C_{12}=$ & $77,84 \mathrm{GPa}$ & $e_{33}=$ & $0 \mathrm{C} / \mathrm{m}^{2}$ & $\sigma_{d}=$ & $50 \mathrm{MPa}$ \\
$C_{13}=$ & $74,28 \mathrm{GPa}$ & $e_{15}=$ & $12,67 \mathrm{C} / \mathrm{m}^{2}$ & & \\
$C_{33}=$ & $115,41 \mathrm{GPa}$ & $\epsilon_{11}=$ & $1474,36 \epsilon_{0} \mathrm{~F} / \mathrm{m}$ & & \\
$C_{44}=$ & $25,64 \mathrm{GPa}$ & $\epsilon_{33}=$ & $1299,41 \epsilon_{0} \mathrm{~F} / \mathrm{m}$ & & \\
$C_{66}=$ & $30,58 \mathrm{GPa}$ & $\epsilon_{0}=$ & $8,854 \times 10^{-12}$ & & \\
\hline
\end{tabular}

Tabela C.5: Propriedades da fibra de carbono com matriz de epóxi (Performance Composite Ltd, ).

\begin{tabular}{rrrrrr}
\hline$E_{1}=$ & $150 \mathrm{GPa}$ & $\nu_{12}=$ & 0,33 & $T_{1}=$ & $2000 \mathrm{MPa}$ \\
$E_{2}=$ & $9 \mathrm{GPa}$ & $\nu_{13}=$ & 0,33 & $C_{1}=$ & $1500 \mathrm{MPa}$ \\
$E_{3}=$ & $9 \mathrm{GPa}$ & $\nu_{23}=$ & 0,5 & $T_{2}=$ & $40 \mathrm{MPa}$ \\
$G_{12}=$ & $6 \mathrm{GPa}$ & & & $C_{2}=$ & $150 \mathrm{MPa}$ \\
$G_{13}=$ & $6 \mathrm{GPa}$ & $\rho=$ & $1500 \mathrm{~kg} / \mathrm{m}^{3}$ & $S=$ & $80 \mathrm{MPa}$ \\
$G_{23}=$ & $3 \mathrm{GPa}$ & & & & \\
\hline
\end{tabular}




\section{Apêndice D - Método de Otimização Topológica}

\section{D.1 Introdução}

O projeto de estruturas envolve, inicialmente, a definição de materiais e uma geometria preliminar que atenda uma série de requisitos de projeto. Em seguida, avalia-se a necessidade de se melhorar o desempenho da estrutura, seja propondo tipos de materiais alternativos, ou modificando sua geometria, de forma a minimizar os esforços e custos na tentativa de que a nova estrutura tenha desempenho similar ou superior ao inicialmente proposto.

Assim, a otimização de estruturas busca melhorar a configuração do projeto de modo a atender aos requisitos de projeto (função objetivo). Como um exemplo para ilustrar esta ideia, considere um problema que consiste em obter uma estrutura com maior rigidez para um dado peso que suporte uma certa carga mecânica aplicada. A Fig. D.1 apresenta três tipos de abordagens para este problema de otimização.

Na primeira abordagem (Fig. D.1(a)) assume-se, por exemplo, uma estrutura formada por elementos de treliça, onde características geométricas, como a área das seções transversais de cada treliça, são definidas como as variáveis de projeto. Essa abordagem é conhecida como otimização paramétrica (VANDERPLAATS, 1984). Assim, aplicando algoritmos computacionais para encontrar as áreas das seções de cada elemento de treliça, obtém-se o resultado apresentado na Fig. D.1(a).

A segunda abordagem (Fig. D.1(b)) é conhecida como otimização de forma (HAFTKA; GURDAL; KAMAT, 1990), onde os contornos internos e externos da estrutura são parametrizados através de curvas splines e os parâmetros dessas curvas assumem o papel das variáveis de projeto. Por meio de algoritmos computacionais, os parâmetros ótimos das curvas splines são determinados e consequentemente, a forma ótima é obtida (ver Fig. D.1(b)). Essa abordagem aumenta o espaço de soluções, sendo um método mais 


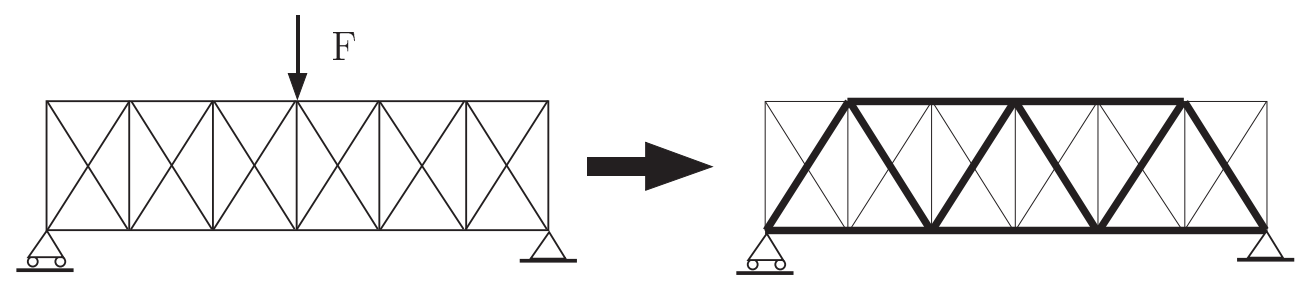

(a) Otimização Paramétrica

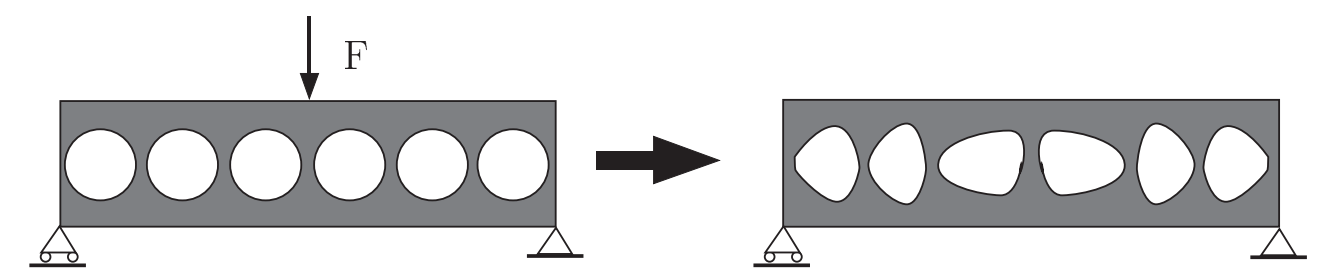

(b) Otimização de Forma

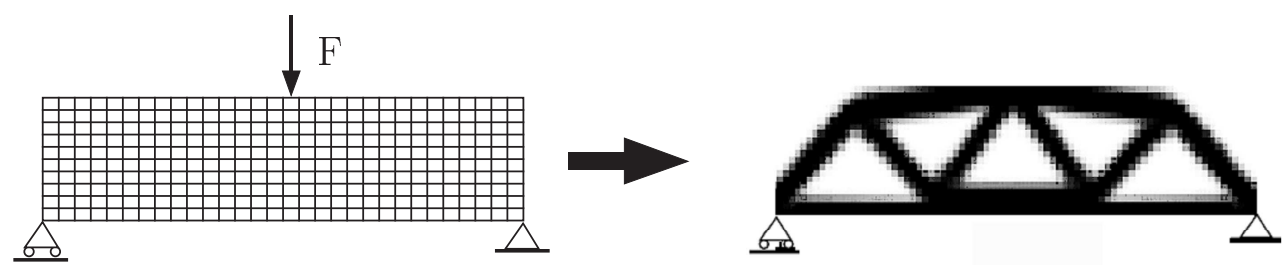

(c) Otimização Topológica

Figura D.1: Exemplo de 3 tipos de otimização estrutural.

genérico que a otimização paramétrica. No entanto, a principal desvantagem deste método é lidar com a contínua mudança de forma do domínio. Se o MEF for utilizado para a análise das estruturas durante o processo de otimização, faz-se necessário utilizar complexos algoritmos de remalhamento, que, se a forma do domínio mudar muito, podem gerar elementos demasiadamente distorcidos.

Finalmente, a terceira abordagem é chamada de Otimização Topológica (OT) que consiste em distribuir material dentro de um domínio fixo (Fig. D.1(c)), permitindo a criação de regiões vazias onde não houver necessidade de material. As variáveis de projeto podem ser, por exemplo, medidas que indicam a quantidade de material em cada ponto do domínio. A OT é a abordagem mais genérica dentre as aqui apresentadas, sendo que a estrutura obtida por OT tende a ser mais leve e com melhor desempenho.

Assim, o Método de Otimização Topológica (MOT) consiste num método computacional que, na sua implementação tradicional, combina métodos de otimização com o MEF e permite, segundo um certo critério de custo, projetar a topologia ótima 
de uma estrutura. Basicamente, o MOT distribui o material no interior de um domínio fixo de forma a maximizar (ou minimizar) uma certa função custo especificada, sujeita a restrições de projeto. Um algoritmo de otimização é utilizado para encontrar a distribuição ótima de material, o que, de forma iterativa, torna o processo rápido, caso contrário seriam necessárias milhares de análises para encontrar a distribuição ótima de material no domínio. Para acelerar o processo de busca da distribuição ótima de material, os métodos de otimização utilizam-se das informações dos gradientes (ou derivadas) da função custo em relação à quantidade de material em cada elemento. Assim, a distribuição de material é representada, por exemplo, associando-se um valor (variável de projeto) a cada ponto do domínio.

\section{D.2 Conceitos do MOT}

Alguns conceitos teóricos da OT devem ser abordados para melhor entendimento da formulação implementada. Os conceitos apresentados a seguir são: domínio fixo estendido, modelo de material e alguns aspectos numéricos do MOT.

\section{D.2.1 Domínio Fixo Estendido}

O domínio fixo estendido (DFE), mais conhecido como domínio de projeto, é o espaço limitado no qual o algoritmo de otimização topológica pode construir a estrutura. A Fig. D.2.1 mostra a DFE, cujos contornos, apoios e carregamentos são fixos e não mudam durante a otimização, e a estrutura otimizada.

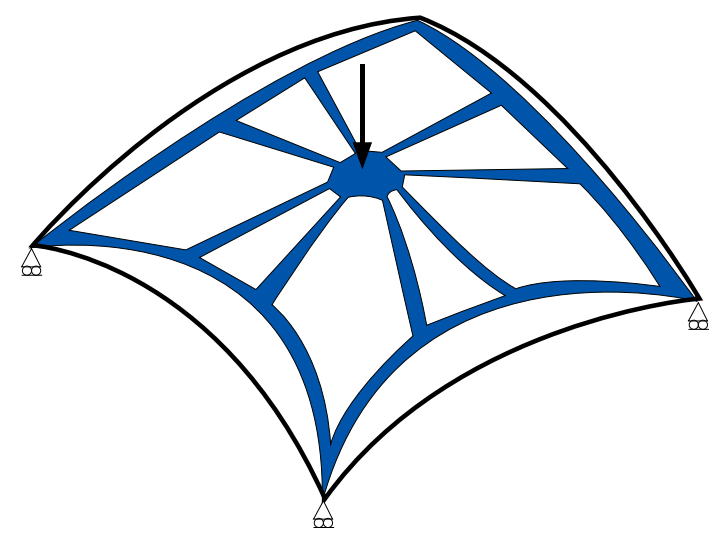

Figura D.2: Conceito de Domínio Fixo Estendido.

Assim, o domínio de projeto deve ser definido pelo projetista no início da análise de modo a definir um espaço de soluções viável de acordo com os requisitos de projeto 
(limitação de dimensões e a geometria). O objetivo da otimização topológica é determinar a distribuição ótima de material influenciada pela função objetivo e restrições de projeto. A grande vantagem em relação aos outros tipos de otimização é que o modelo de elementos finitos do domínio não é alterado durante o processo de otimização, simplificando a análise estrutural em cada iteração.

\section{D.2.2 Modelo de Material}

Para que a OT possa encontrar a estrutura ótima dentro da DFE, deve-se primeiramente parametrizar a distribuição de material de modo que uma função $\chi(\Omega)$ possa determinar se cada ponto do domínio deve ser sólido ou vazio. A função $\chi(\Omega)$ pode ser definida da seguinte maneira:

$$
\chi(\Omega)= \begin{cases}1, & \text { presença de material } \\ 0, & \text { ausência de material }\end{cases}
$$

sendo que essa função deve ser válida para qualquer ponto dentro do domínio $\Omega$ definido pela DFE. A função $\chi(\Omega)$ é discreta, ou seja, possui somente valores 1 ou 0 , para presença ou ausência de material, respectivamente.

Entretanto, a distribuição discreta $(0-1)$ de material resulta em um problema que não possui solução única (SIGMUND; PETERSSON, 1998), pois depende da discretização da malha. Assim, a função $\chi$ deve ser relaxada de modo a permitir valores intermediários entre 0 e 1 durante o processo de otimização, garantindo a unicidade de solução. Num projeto onde se deseja a distribuição ótima de material podendo haver buracos na estrutura, os estágios intermediários de material não têm significado físico, sendo apenas um recurso matemático decorrente da relaxação do problema, inviabilizando sua fabricação. Portanto, é necessário utilizar uma penalização nos valores intermediários de forma a recuperar o caráter discreto ao final da otimização.

Então, é introduzido o conceito de modelo de material que, essencialmente, é uma equação que define a mistura em micro-escala de dois materiais, sendo que um deles pode ser "vazio". Existem basicamente duas abordagens para modelos de materiais relacionados à distribuição de material: modelos baseados em microestrutura, que se baseiam na existência de uma microestrutura em cada elemento cuja geometria é parametrizável, como por exemplo o método da homogeneização (BENDSøE; KIKUCHI, 1988; NISHIWAKI et al., 2001), e os modelos chamados de artificiais, que definem uma função matemática contínua de modo a variar as propriedades dos materiais entre 0 e 1 em cada ponto do 
domínio, como por exemplo o "Solid Isotropic Material with Penalization" (ou SIMP) (BENDSøE, 1989; ROZVANY; ZHOU; BIRKER, 1992).

\section{D.2.3 Aspectos Numéricos do MOT}

Neste item são apresentados os aspectos numéricos mais relevantes do método de otimização topológica que, se não forem contornados, comprometem a solução final do problema.

O primeiro aspecto numérico é decorrente da relaxação da função densidade volumétrica, na qual ela assume valores entre 0 e 1. Em geral se escolhe a cor branca para representar a ausência de material (valor 0) e preto para representar o presença (valor 1), sendo que os valores intermediários são representados por uma escala gradativa de cinza que varia do branco ao preto. Por isso, este aspecto numérico é comumente chamado de escala de cinza. Uma representação da escala de cinza pode ser vista na Fig. D.3.

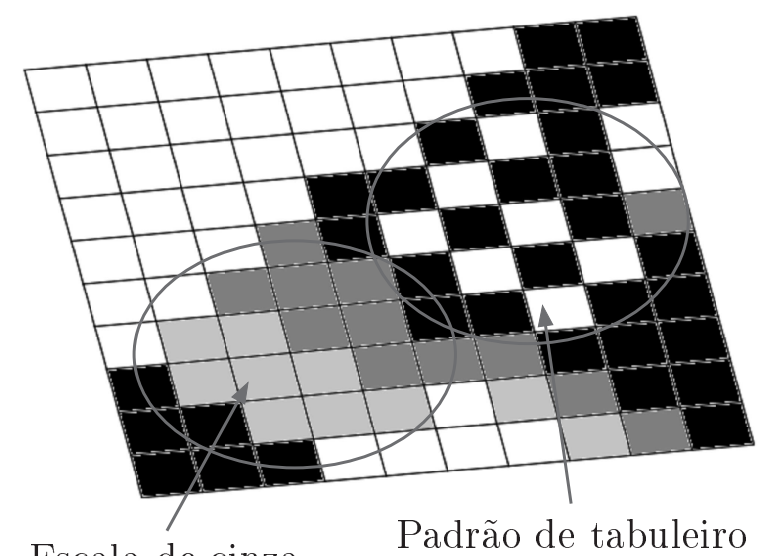

Escala de cinza

Padrão de tabuleiro

Figura D.3: Representação de um resultado com escalas de cinza e o padrão de tabuleiro.

O meio mais comum de se tratar a escala de cinza é utilizando as penalizações no modelo de material, que faz com que os valores intermediários se aproximem dos limites 0 ou 1, recuperando o caráter discreto da distribuição de material. Entretanto, essa abordagem não garante que os resultados não apresentarão escala de cinza (STOLPE; SVANBERG, 2001).

O segundo aspecto numérico é decorrente do problema discreto 0-1, no qual a solução depende da discretização da malha. Este problema é conhecido na literatura como dependência de malha, que, conforme a malha utilizada é refinada, mais espaços vazios surgem no domínio, fazendo com que as estruturas fiquem demasiadamente delgadas, como representado na Fig. D.4. Entretanto, essa dependência aparece também quando 
se utilizam modelos de material penalizados, que no final recuperam o caráter discreto da solução. Para contornar esse problema, podem ser aplicadas algumas técnicas como a de restrição de perímetro (AMBRosio; BUTTAzzo, 1993), filtros no campo das pseudo-densidades (BOURDIN, 2001; SWAN; KOSAKA, 1997), filtro nos gradientes (SIGMUND, 2001), ou técnicas de projeção (BRUNS; TORTORELLI, 2001; GUEST; PREVOST; BELYTSCHKO, 2004; GUEST; ASADPOURE; HA, 2011).

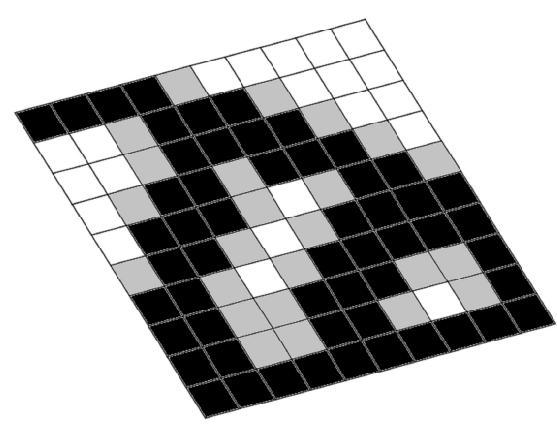

(a) Malha $10 \times 10$

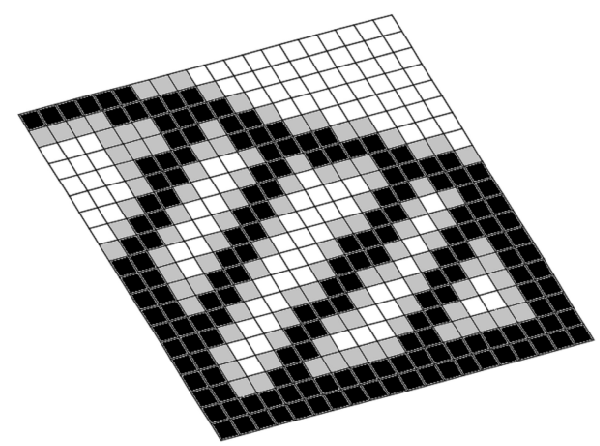

(b) Malha $20 \times 20$

Figura D.4: Representação de um exemplo onde a solução depende do refinamento da malha.

O terceiro aspecto numérico relevante é a instabilidade de tabuleiro, cuja característica é formar uma estrutura onde elementos adjacentes apresentam presença e ausência de material, dispostos em forma de tabuleiro, como mostrado na Fig. D.3. Diaz e Sigmund (1995) e Jog e Haber (1996) constataram que a instabilidade de tabuleiro é um fenômeno inerente a problemas de variacionais mistos. O problema de OT utilizando métodos das densidades pode ser interpretado como um problema que contém um variacional misto envolvendo o campo de densidades. Assim, mesmo utilizando elementos de alta ordem (9 nó por exemplo) ainda é observada a presença desses padrões de tabuleiro (JOG; HABER, 1996). A formação dos padrões de tabuleiro não é um aspecto que aparece somente em problemas de OT. Essa instabilidade também é observada na solução de MEF para diversos outros problemas com variacional misto, como por exemplo, na solução do MEF para obter a distribuição de pressões num problema de escoamento de fluidos de Stokes (ODEN; KIKUCHI; SONG, 1982), cujo variacional envolve velocidades e pressões.

Em problemas de OT, a instabilidade de tabuleiro se caracteriza pelas variações bruscas nos gradientes das variáveis de projeto. A abordagem mais utilizada na literatura para evitar o padrão de tabuleiro é a implementação de filtros especiais na formulação e OT (BENDSøE; SIGMUND, 2003), que introduz métodos de controle da variação espacial das 
pseudo-densidades no domínio de projeto. Além disso, essa abordagem também permite controlar a complexidade da topologia obtida pelo MOT (BOURDIN, 2001), bem como a dependência de malha. Sigmund (2007) apresenta uma análise dos métodos utilizados para reduzir os problemas numéricos da OT e apresenta uma revisão completa dobre esse assunto, e sugere a utilização da técnica de projeção de Bruns e Tortorelli (2001). 